\title{
ABSTRACTS OF THE TENTH ANNUAL MEETING OF THE
}

\section{ISRAEL SOCIETY FOR NEUROSCIENCES}

Eilat, Israel, December 16 - 18, 2001

\begin{abstract}
Writing the stroop-effect: Color naming with handwritten
responses ${ }_{\text {Adi-Japha E. }}^{1,2}$ and Tzelgov J. ${ }^{3}$

Collage Of Judea and Samaria. 2Bar-Ilan University, Ramat

Gan; 3Ben-Gurion University of the Negev, Beer Sheva

We investigated the coupling between response selection and execution in a Stroop task with handwritten responses. The studied coupling is between the input level of the motor system controlling handwritten response (Margo in, Quarterly Journal of Experimental Psychology, 34A: 459-489 [1984]), and the lingual levels that serve as the output of the response selection stage (Cohen et al, Psychological Review 97: 332-361 [1990]). Incorrect responses, indicating processing of both the word meaning and the color (e.g., 'rblue' in response to the word "RED" written in blue), were typical in the incongruent condition. The Stroop effect was significant when comparing the congruent and incongruent conditions: the former was written more fluently and its execution time varied less. The effect was even stronger in the first letter. In line with the literature, there was no difference in the mean execution time in the two conditions (Logan and Zbrodoff, Journal of Experimental Psychology: HPP 24(3): 978992 [1998]). These results are explained by a model of an ongoing selection process that runs in parallel to an execution process. According to the model, the conflicting information arriving from the intended processing of color and the (unintended) automatic reading finds its expression either in an incorrect response or in an increased variation in the execution time, especially in the first written letter.

Keywords: Stroop, Response selection, Response execution,
\end{abstract} Writing.

Subthreshold correlates of comodulation masking release in cat primary auditory cortex

Ahdut L. ${ }^{1}$ Stern E.A. ${ }^{2}$ and Nelken ${ }^{1}{ }^{1}$

IDept. of physiology, Hebrew University - Hadassah Medical School, and the Interdisciplinary Center for Neural Computation, Hebrew University, Jerusalem, Israel; 2Dept. of Neurology, Massachusetts General Hospital, Harvard Medical School, Charlestown, MA, USA

Comodulation Masking Release (CMR) is a psycho-acoustical phenomenon, in which amplitude modulation of a masking noise causes a decrease in its ability to mask tones. We have previously described an analogue of CMR in neuronal activity in cat primary auditory cortex (AI). Whereas neuronal firing locked to the envelope of the noise when presented alone, the addition of a weak tone suppressed this locking. This is an example of an extremely non-linear spectro-temporal integration mechanism.

In order to understand the mechanisms underlying this phenomenon, we performed intracellular recordings in vivo. The data were collected in 7 halothane-anesthetized cats, using sharp electrodes filled with either $\mathrm{KCl}$ or KAcetate. Neurons were held up to 2-3 hours. The subthreshold membrane potential was locked to the temporal envelope of the noise when presented alone, while the addition of a low-level tone suppressed the locking. Thus, the spike responses could be fully accounted for by the subthreshold responses. To further discover the underlying mechanisms of synaptic integration responsible for this effect, responses to the same stimuli were recorded while the cell was depolarized by steady current injections. Whereas some inhibitory components were uncovered by depolarization, most of the components of the subthreshold responses were not qualitatively changed. Thus, our results suggest that the strong effects of a weak tone on the responses to a strong noise stimulus are already present at the excitatory input to AI neurons. Extracellular recordings in the thalamic input station to $\mathrm{AI}$, the medial geniculate nucleus, support this conclusion.

Keywords: Comodulation masking release, Intracellular, Auditory cortex.
Amygdala complex modulation of hippocampal long-term potentiation

Akirav I. and Richter-Levin G.

Dept. of Psychology, University of Haifa, Haifa 31905

The amygdala is considered to be involved in the storage of emotionally arousing events. We have shown (Akirav and RichterLevin, 1999) that activating the basolateral amygdala (BLA) prior to stimulation of the perforant path (PP) may have a bi-phasic effect on hippocampal plasticity: priming the BLA immediately prior to PP stimulation results in the enhancement of hippocampal long-term potentiation (LTP), whereas BLA spaced activation results in the suppression of hippocampal LTP. We suggested that the enhancing effect may serve as a marker for emotional experiences (an 'emotional tag'), and the inhibiting effect may be beneficial in reducing masking effects of subsequent, lesssignificant events during the initial stages of consolidation.

Here, we examined the involvement of noradrenaline (NA) and glucocorticoids (GLUC) as possible mediators of the fast and slow phases, respectively. Ipsilateral priming of the BLA significantly enhanced hippocampal LTP, confirming previous results. This enhancing effect was not found in NA-depleted rats, indicating that amygdala-enhancing modulation of hippocampal LTP is dependent on noradrenergic activation. As was shown previously, Ipsilateral BLA spaced activation significantly suppressed hippocampal LTP. We have started to examine whether corticosterone depletion abolish this effect.

Activating the Contralateral BLA showed a different profile; priming the Contralateral BLA produced enhancement of LTP but this was not sensitive to NA depletion. In addition, spaced activation of the Contralateral BLA did not result in the suppression of LTP. The results suggest that the Ipsilateral and the Contralateral BLA may differentially influence hippocampal activity, adding to the complexity presumably required to establish an emotionally enriched memory.

Supported by The Israel Science Foundation, Charles H. Revson Foundation (no.582/00-1 to G.R-L.).

Keywords: LTP, Amygdala, Norepinephrine, Glucocorticoids.

The Pore region links fast and slow gating in inwardly rectifying potassium channels

Alagem N., Yesilevsky S., and Reuveny E.

Dept. of Biological Chemistry, Weizmann Institute of Science, Rehovot

Inwardly rectifying potassium channels $\left(\mathrm{K}_{\mathrm{in}}\right)$ are involved in many physiological actions, such as setting the state of excitability of nerve and muscle, potassium secretion and insulin release. Despite the high homology between the various members in this channel family, they display a wide range of single channel gating behavior. The molecular elements which control single channel gating of ion channels, are poorly understood. In an attempt to understand the process of single channel gating, we used the Kir channel family, which possesses a prototypical $\mathrm{K}^{+}$channel structure. We generated chimeras between two Kir channels that display radically different single channel kinetics: Kir2.1 and Kir3.1/3.4. Single Kir2.1 channels have a high probability of opening $(\sim 0.8)$ and are typically open for hundreds of milliseconds. On the other hand, Kir3.1/3.4, which composes the atrial muscarinic channel of the heart, has a very low probability of opening $(-0.05)$. Kir3.1/3.4 openings are only a few milliseconds in duration and are clustered into bursts. Using a chimeric approach, we found two amino acid residues in the pore which control both single channel open time and burst duration, in Kir3.1/3.4. The pore mutants displayed wild-type levels of carbachol gating, suggesting that they affect a gate which is distinct from the $G \beta \gamma$ gate. We have used our results to construct a model of the Kir3.1/3.4 pore, which describes the energy of interaction of permeating $\mathrm{K}^{+}$ions with the channel.

Keywords: Ion channels, Gating, Patch clamp 
Object-related activation in different modalities (visual, tactile and auditory) in the human occipital and temporal cortex Amedi A. ${ }^{1}$, Hendler T. ${ }^{2}$, Malach R. ${ }^{3}$ and Zohary E. ${ }^{1}$ Dept. of Neurobiology, Life Science Institute and Neural Computation Center, Hebrew University, Jerusalem 91904; ${ }^{2}$ Wohl Institute for advanced imaging, Tel-Aviv Sourasky Medical Center, Itel Aviv; ${ }^{3}$ Dept. of Neurobiology, Weizmann Institute of Science, Rehovot 76100

Object recognition in humans and primates has been shown to depend on processing in the ventral visual stream. We have recently demonstrated that part of the human ventral visual pathway, located within the lateral occipital complex (LOC), is activated by both visual and haptic stimulation (Amedi et al Nat. Neurosci. 4, 324-330 [2001]). The characteristic feature of this region is that in both visual and tactile modalities, objects elicit stronger activation than textures. We show here that this object selectivity is robust and repeatable in both modalities across subjects as well as across scans. In contrast, auditory sounds characteristic of objects (animals, tools, vehicles, etc) do not evoke any responses in this region. Thus, we term this bi-modal area SOLO for Somatosensory Object-related Lateral Occipital. We suggest that SOLO is involved in the analysis of physical shape, which can be acquired from both visual and haptic sources. The level of representation of physical shape in this region is not clear enough and can be from the level of basic feature to the level of the 3D model of the objects. In contrast, auditory information contributes little to the reconstruction of physical shape. This may explain the lack of auditory activation in SOLO. On the other hand, voxels in regions around the superior-temporal sulcus were significantly activated by auditory objects compared to control noise.

Keywords: Objects, Multimodal, fMRI, Ventral stream.

\section{Novel bifunctional compounds eliciting cholinergic and anti- inflammatory activity for the treatment of CNS impairments} Amitai G., Adani R., Rabinovitz I., Sod-Moriah G. and Meshulam H. Division of Medicinal Chemistry, Israel Institute for Biological Research, P.O. Box 19, Ness Ziona, 74100

The development of new drugs for treatment of various CNS degenerative diseases such as dementia of Alzheimer's type (AD) is based mainly on the use of cholinergic compounds such as cholinesterase inhibitors (ChEI). Anti-inflammatory drugs were shown to ameliorate the inflammatory processes associated with these diseases. The only clinically used drugs, which presently demonstrate efficacy in mild to moderate $\mathrm{AD}$, are ChEIs (e.g., Aricept, Exelon and Reminyl). It was also indicated that certain non-steroidal anti-inflammatory drugs (NSAID) could be used for prevention of AD.

We have synthesized a series of novel bifunctional compounds that contain covalently coupled cholinergic up-regulators (CURE) such as quaternary ChEIs, muscarinic and nicotinic agonists with various NSAIDs. A hydrophobic linker connects between these moieties and facilitates permeability through the BBB. We report here on bifunctional NSAID-CURE conjugates that contain PYR as ChEI moiety coupled via an octyl $\left(\mathrm{C}_{8} \mathrm{H}_{16}\right)$ linker (PO) to one of the following NSAIDs: ibuprofen (IBU), diclofenac (DICLO), indomethacine (INDO), aspirin (ASP) and naproxen (NAP). IBUPO, DICLO-PO, INDO-PO, ASP-PO and NAP-PO inhibit both $\mathrm{AChE}$ and $\mathrm{BChE}$ with dissociation constants $\left(\mathrm{K}_{\mathrm{I}}\right)$ and bimolecular inhibition rate constants $\left(\mathrm{k}_{\mathrm{i}}\right)$ that range between $10^{-6}-10^{-7} \mathrm{M}$ and $10^{5}-10^{6} \mathrm{M}^{-1} \mathrm{~min}^{-1}$ respectively. Some of these bifunctional compounds also inhibit cycloxygenase (COX I and COX II) at micromolar level. Thus, the NSAID-ChEI conjugates exert both anti-ChE and anti-COX activity at equi-molar concentrations even in their intact non-hydrolyzed form. The acute toxicity of these compounds is $10-20$ fold lower than that of PYR with $\mathrm{LD}_{50}$ values that range at $50-100 \mathrm{mg} / \mathrm{kg}$ ip in mice. Anti-inflammatory activity of IBU-PO was examined in carrageenan-induced peripheral and CNS inflammation in rats. Pretreatment with IBU-PO $(5 \mathrm{mg} / \mathrm{kg}$, ip) decreased significantly the rat paw edema level and whole brain edema. IBU-PO $(5 \mathrm{mg} / \mathrm{kg}$, ip) increased by 8 -fold the survival time of mice that were exposed to hypobaric hypoxia as compared to control animals. IBU-PO $(5 \mathrm{mg} / \mathrm{kg}$, ip) decreased significantly the brain edema and improved the neurological severity score in closed head injury model in mice. These findings suggest that the new NSAID-ChEI bifunctional chimers could be useful for treatment of CNS impairments such as cerebro-vascular dementia and for reducing the neuronal damage caused by either acute cerebral ischemia or closed head injury

Keywords: Cholinesterase inhibitors, Non-steroidal antiinflammatory drugs, Neurodegenerative diseases

Disabled readers do not have a specific magnocellular deficit Amitay S., ${ }^{1}$ Ben-Yehudah $\mathrm{G}_{\text {, }}{ }^{2}$ Banai K., ${ }^{1}$ and Ahissar M. ${ }^{1,3}$ Interdisciplinary Center for Neural Computation ${ }^{1}$, Dept. of Neurobiology ${ }^{2}$, and Dept. of Psychology, Hebrew University Jerusalem

The magnocellular theory is a prominent, albeit controversial, view asserting that many reading disabled $(R D)$ suffer from a specific impairment in the visual magnocellular pathway. This theory is a part of a more general theory suggesting that RDs have a pansensory deficit in fast temporal processing. In order to assess the validity of the magnocellular theory we tested its two basic predictions. First, that a sub-population of $\mathrm{RDs}$ will show impaired performance across a broad range of psychophysical tasks relying on magnocellular functions. Second, that this subpopulation will not be consistently impaired across tasks that do not rely on magnocellular functions. We defined a behavioral criterion for magnocellular function, which incorporates performance in flicker detection, detection of drifting gratings (at low spatial frequencies), speed discrimination of drifting gratings and detection of coherent dot motion. We found that some RDs $(6 / 30)$ had impaired magnocellular function. Yet, RDs who were consistently impaired on magnocellular tasks were also consistently impaired on a broad range of other visual and auditory perceptual tasks. Moreover, their performance did not improve with longer stimulus durations. We conclude that although some RDs have poor perceptual abilities, the "magnocellular" level of description does not capture the essence of the perceptual difficulties of any $\mathrm{RD}$ individual we have assessed.

Keywords: Magnocellular, Reading disability, Temporal processing, Perception.

\section{Nanoparticle-based EM-localization of acetylcholinesterase in} vertebrate neuromuscular junctions

Anglister L., Blotnick E. and Sharon S.

Dept. of Anatomy \& Cell Biology, Hebrew University Hadassah Medical School, Jerusalem 91120

Acetylcholinesterase (AChE) is concentrated in cholinergic synapses, where it is a major factor controling the duration of transmitter action. The proper function of synaptic $\mathrm{AChE}$ is determined by its concentration and position in the synaptic cleft. The densities of synaptic AChE at various neuromuscular junctions (nmjs) were evaluated by quantitative EMautoradiography with radio-labeled probes. However, the precise distribution and location of the enzyme in the cleft could not have been determined. Thus, it is not clear whether and to what extent synaptic AChE is associated with pre- or post synaptic membranes, or with synaptic basal lamina, and whether it is distributed only in the primary cleft or also in postjucntional folds. The present study describes nanoparticle-based EM-localization of $\mathrm{AChE}$ in the synaptic cleft of vertebrate nmjs. Various nanogoldconjugates of fasciculin, a polypeptide anticholinesterase toxin, were prepared and used to label AChE in nmjs of mouse and frog muscles. Nanogold labeling was very effective. Intense nanogoldlabeling was obtained even at frog nmjs, where AChE site-density is low relative to other vertebrates. Gold-labeled AChE sites were distributed over the basal lamina in the primary cleft and the postjunctional folds. Quantitative data analysis demonstrates that AChE sites are almost exclusively located on the basal lamina rather than pre- or postsynaptic membranes and are distributed in the primary cleft and full depth of the postjunctional folds, with a defined pattern. This localization pattern of $\mathrm{AChE}$ assures the hydrolysis of $\mathrm{ACh}$ bouncing off receptors, and eliminates its unnecessary rebinding.

Supported by the Israel Sci. Found. - Israel Acad. Sci. 180/98

Keywords: Nanogold, Acetylcholinesterase, Basal lamina, Synaptic cleft 
DP-109, a lipophilic transition metal chelator, attenuates asymmetric rotations in the 6-OHDA partial lesion model of Parkinson's disease in the adult rat Aran A., Sorant N., Kozak A., Shapiro I., Friedman N., Angel I. and Friedman J.E.

D-Pharm Ltd, Kiryat Weizmann Science Park, Rehovot 76123

Over accumulation of transition metals such as copper, iron and zinc can cause oxidative stress in neurons. These metals are considered to be involved in neurodegenerative disorders such as Parkinson's and Alzheimer's disease. Antioxidants and metal chelators have been found to be beneficial in various models of these diseases, but are problematic due to poor penetration across the blood brain barrier. To address this problem, we have developed a family of lipophilic transition metal chelators. The lead compound, DP-109 has $\mathrm{K}_{d}(\mathrm{Ca}) \sim 5 \times 10^{-5} \mathrm{M}$, whereas the $\mathrm{K}_{d}(\mathrm{Cu}) \sim 10^{-9} \mathrm{M}$ and is highly lipophilic, with $\log _{\mathrm{p}}>2.5$ (octanol/water). DP-109 was tested in the unilateral 6-OHDA substantia nigra lesion model using adult male Wistar rats. Three days post-lesion animals were screened for apomorphine-induced rotations over a $5 \mathrm{~min}$ period. Responding animals subsequently received either DP-109 (10-500 $\mu \mathrm{g} / \mathrm{kg})$ or vehicle daily, p.o., for a period of up to 60 days. Animals were tested weekly for apomorphine-induced rotations. Results were normalized and the increase in asymmetric rotations, indicating progressive neurodegeneration, was recorded. After 1 or 2 months brains were taken for morphologic analysis. Vehicle treated animals increased their rotations approximately 20 -fold within $30 \mathrm{~d}$. DP-109 treated animals demonstrated significantly fewer rotations in a dosedependant manner. DP-109 attenuated both the rate of increase and number of rotations by $75 \%$. Morphologic analysis found unilateral damage along the striatum in all animals. DP-109treated animals exhibited less neuronal loss than seen in vehicle controls. We suggest that DP-109 represents a new class of compounds that might be effective in treating neurodegenerative disorders.

Keywords: Neurodegenerative disorders, Parkinson's disease, Chelators, Drugs

Lithium, a neuroprotective drug in MPTP mice model of Parkinson's disease Arraf Z. and Youdim M.B.H

Eve Topf and NPF Centers of Excellence For Neurodegenerative Diseases Research and Dept. of Pharmacology, Rappaprt Faculty of medicine, Technion, Haifa

Lithium is employed successfully for treatment of bipolar depression. However its mechanism of action has not been fully established. Recent studies by others have indicated that lithium may be a neuroprotective drug and this has been attributed to its ability to induce the anti apoptotic mitochondrial protein, Bcl-2 Since Bcl-2 over expression in transgenic mice and PC-12 cells prevents MPTP and N-methyl-( $R$ )- salsolinol neurotoxicity respectively we examined lithium's neuroprotective activity in MPTP (N-methy-4-phenyl-1,2,3,6-tetrahydropyridine) mice model of Parkinson's disease. Mice were fed with diets containing either 2.2 or $3.3 \mathrm{gr} / \mathrm{kg}$ of lithium chloride for 28 days . They were treated with MPTP $(24 \mathrm{mg} / \mathrm{kg} /$ day $\times 4$ days) and killed 4 days later and striatal dopamine metabolism and turn over were estimated. The two doses of lithium gave serum lithium concentrations of 0.78 and $0.98 \mathrm{mM}$.. Both doses of lithium induced neuroprotection against MPTP as determined by prevention of the fall in striatal dopamine, DOPAC and HVA, with higher dose being more effective The confirmation of lithium's neuroprotective activity has come from turn over of striatal dopamine metabolism (DOPAC + HVA / dopamine).The mechanism of lithium induced neuroprotective activity and its relationship to $\mathrm{Bcl}-2$ will be presented.

Keywords: Lithium, MPTP, Neuroprotection, Dopamine neuron, Bcl-2

The role of Munc13-1 in neurotransmitter release and hormone secretion.

Ashery U.

Dept. of Neurobiochemistry, Wise Faculty of Life Sciences, Tel Aviv University, Tel Aviv

Neurotransmitter release involves vesicle docking, priming, fusion and recycling. These processes are coordinated by a large number of synaptic proteins and depend on proper protein-protein and protein-lipid interactions. Munc13-1 is a presynaptic protein that interacts with syntaxin, Doc2, Munc18 and RIM and is essential for a post-docking step in the synaptic vesicle cycle. To investigate whether Munc13-1 acts as a priming factor, we overexpressed Munc13-1 in adrenal chromaffin cells using the Semliki Forest Virus system. Using flash photolysis of cagedcalcium, we studied the effect of Munc13-1 on the different kinetic components of exocytosis with capacitance measurements and electrochemical detection of catecholamine release. Overexpression of Munc13-1 causes a 3-fold increase in the exocytotic burst, which represent the fusion of release-competent vesicles. Furthermore, it accelerates the sustained component of secretion, which represents vesicle priming and fusion. Since there was no apparent change in the number of docked vesicles, we conclude that Munc13-1 acts as a priming factor for large dense core vesicles by accelerating the rate constant of vesicle priming. The enhancement of secretion by Munc13-1 ${ }^{\mathrm{H} 56 \% \mathrm{~K}}$, which carries a point mutation in the $\mathrm{Cys}_{6} \mathrm{His}_{2}$ motif of the $\mathrm{C}_{1}$ domain and therefore no longer binds phorbol ester, was less pronounced. Interestingly, the C-terminal part of Munc13-1 enhances secretion in a similar way to the wild-type Munc13-1. These data suggest that the $C_{1}$ domain facilitates the action of Munc13-1 and that the priming activity of Munc13-1 resides on the its C-terminal part. Possible models for vesicle docking, priming and fusion will be discussed.

Keywords: Neurotransmission, Capacitance, Munc13-1,

Chromaffin cells.

Imaging of demyelination in multiple-sclerosis using q-space diffusion and spectroscopic magnetic resonance imaging Assaf Y. , Chapman J. ${ }^{2}$, Ben-Bashat D. ., Segev Y. ', Graif M. ${ }^{1}$, Hendler T. ', Korczyn A.D. ${ }^{2}$ and Cohen Y.

${ }^{1}$ Wohl Institute for Advanced Imaging, Sourasky Medical Center, Tel Aviv, Israel, ${ }^{2}$ Dept. of Neurology, Sourasky Medical Center, Tel Aviv, Israel; ${ }^{3}$ School of Chemistry, Tel Aviv University, Tel Aviv

Multiple sclerosis (MS) is an inflammatory disease of the central nervous systems leading to progressive decline of motor and sensory functions and causing permanent disability. Magnetic resonance imaging (MRI) is well established as a diagnostic imaging method in MS showing multiple hyper-intense lesions in T2 and FLAIR images (MS plaques) and hypo-intense lesion on T1 weighted images ("black holes"). N-acetyl-aspartate (NAA) levels, as measured from magnetic resonance spectroscopy (MRS) revealed much larger abnormal areas than those detected by the conventional MRI methods. These abnormal areas were termed as normal appearing white matter (NAWM). Recently, we have showed that high $b$ value diffusion MRI, analyzed using the qspace approach, detect more extensive white matter (WM) abnormality in MS brains as compared to conventional MRI methods.

In this study we performed a correlation between high $b$ value diffusion MRI and ${ }^{1} \mathrm{H}$ 2D MR spectroscopic imaging on MS patients. We have used the ${ }^{1} \mathrm{H}$ MRS spectra and conventional MRI to characterize areas of MS lesions, areas of NAWM and areas of normal white matter (normal MRI intensity and normal metabolite distribution in the MRS). According to this differentiation, the average $\mathrm{NAA} / \mathrm{Cr}$ ratios were found to $2.41 \pm 0.37$ and $1.70 \pm 0.25$ for normal WM and NAWM, respectively in MS patients. In these areas, we found that $\mathrm{q}$-space probability images intensity was significantly different between the group of NAWM and normal white matter $(7.43 \pm 0.64$ and $6.70 \pm 0.57$, respectively, $\mathrm{p}<0.001)$. This study showed that q-space diffusion MR imaging provides images that are in high correlation to the metabolic state of tissue and therefore show high sensitivity to early abnormal processes in WM.

Keywords: Magnetic resonance imaging, Magnetic resonance spectroscopy, Diffusion, Multiple sclerosis, N-Acetyl-Aspartate

Availability of somato-dendritic $\mathrm{Na}^{+}$channels in layer 5 neocortical neurons is tuned by membrane potential oscillations

Astman N. ${ }^{1}$, Gutnick M.J. ${ }^{2}$ and Fleidervish I.A. ${ }^{2}$

'Zlotowski Center for Neuroscience, BenGurion University of the Negev, Beersheva \& ${ }^{2}$ Koret School of Veterinary Medicine, Hebrew University of Jerusalem, Rehovot 76100

Back-propagating action potentials in Layer 5 pyramidal neurons may provide the postsynaptic dendritic depolarization necessary for the induction of long-term synaptic plasticity. In order to back-propagation to occur, $\mathrm{Na}^{+}$channels must be available. We used cell-attached configuration of patch-in-slice technique to examine the availability and dynamic characteristics of $\mathrm{Na}^{+}$ 
channels in neocortical Layer 5 cells. At physiological temperature $\left(35-37^{\circ} \mathrm{C}\right) \mathrm{Na}^{+}$channel steady-state inactivation $\left(\mathrm{h}_{\infty}\right)$ appeared to be highly voltage-dependent $\left(7.2 \pm 0.4 \mathrm{mV}^{-1}\right.$ vs. 9.7 $\pm 0.4 \mathrm{mV}^{-1}$ at $\left.22^{\circ} \mathrm{C}, \mathrm{p}<0.05, \mathrm{n}=7\right)$, with a midpoint at $\mathrm{V}_{\text {rest }}{ }^{-3} \pm 2$ $\mathrm{mV}$. At voltages around $\mathrm{V}_{\text {rest }}$, the time constant of inactivation, as measured with double-pulse reactivation protocol, was unexpectedly slow $(8-12 \mathrm{~ms}, \mathrm{n}=12)$. To determine how neuronal oscillatory behavior may affect the availability of $\mathrm{Na}^{+}$channels, sinusoidal voltage commands of constant amplitude $( \pm 5 \mathrm{mV}$ from $V_{\text {rest }}$ ) at various frequencies (from 5 to $100 \mathrm{~Hz}$ ) were applied to the patches; the amount of "ready-to-open" channels was measured by applying a brief depolarizing pulse from the positive edge of sine wave. We discovered that $\mathrm{Na}^{+}$channel availability is lowest at $5 \mathrm{~Hz}$, and it increases sharply as a function of frequency reaching maximal value at frequencies of $>30 \mathrm{~Hz}$. Our evidence strongly indicates that, in individual Layer 5 neurons, the availability of $\mathrm{Na}^{+}$channels and dendritic excitability could be tuned by frequency and phase of circuit-driven oscillations.

Supported by a grant from the German-Israeli Foundation for

Scientific Research and Development.

Keywords: Na channel; Inactivation; Neocortical neuron; Oscillations

Revealing functional "hot spots" at sub-voxel fMRI resolution and their relevance to cortical modularity.

Avidan G. ${ }^{1}$, Hasson U. ${ }^{2}$, Hendler T. ${ }^{3,4}$, Zohary E. ${ }^{1}$, and Malach R. ${ }^{2}$ ${ }^{7}$ Hebrew University of Jerusalem, Jerusalem $91904 ;{ }^{2}$ Weizmann Institute of Science, Rehovot 76100; ${ }^{3}$ Sourasky Medical Center, Tel Aviv 64239; ${ }^{4}$ Tel Aviv University, Tel Aviv, 69978

High fMRI signals are commonly associated with strong and selective neuronal activity and consequently weak signals are underestimated. However, weak fMRI signals could also stem from intense neuronal activity if it is produced by small groups of neurons ("hot spots") within a voxel. Conventional fMRI resolution is too coarse to reveal such activity. We propose to circumvent this limitation by employing the fMRI-adaptation approach. We first show that fMRI adaptation is dependent on strong neuronal activation. 9 Subjects viewed line-drawings of faces and cars in 3 contrast levels presented in blocks containing either different or identical stimuli. In high order, object-related areas (LO and $\mathrm{pFs}$ ) adaptation was abolished when neurona activation was reduced due to lowering of image contrast. We then used the adaptation method to study the neuronal selectivity in the fusiform face area. While the overall activity in this area was drastically reduced for non-face stimuli (houses and words), the adaptation level obtained for these stimuli remained high. This indicates the existence of small neuronal "hot-spots", which are selective for non-face stimuli. We propose the fMRI-adaptation approach as a general tool for exposing neuronal selectivity below the spatial resolution of conventionally measured fMRI

Funded by Israel Academy 8009/00-1 grant

Keywords: Object recognition, Visual cortex, Ventral stream.

Early exposure to stress modulates the ability to cope with stress in adulthood, in the rat

Avital A., Jacobson S., Yizraeli O. and Richter-Levin G.

Dept. of Psychology, University of Haifa, Haifa

Recent evidence supports the hypothesis that impoverished intellectual stimulation during early childhood may disturb the formation of functional brain pathways, in particular of the limbic circuits, which play a major role in emotion and learning.

Thus, we examined the effects of early exposure to stress on the ability to cope with stress in adulthood. Rats were subjected to platform stress (for 30 minutes) pre-puberty (4 weeks). Postpuberty, at the age of 8 weeks, rats were exposed to adulthood stress experience (Host-intruder interaction), and were then tested in the open-field, the Morris water-maze and the startle-reflex test. We report that an early exposure to stress led to elevated levels of anxiety, as measured in an open-field test, but that rats that were exposed to early + late stress showed significantly higher level of anxiety compared with all groups. Basal startle response was also elevated. Nevertheless, the early + late stress group acquired faster the spatial learning task, though following acquisition, only the controls showed a bias towards the quadrant in which the platform was previously placed.

These findings suggest that an early exposure to stress may have significant and differential effects on behavior and on the ability of rats to cope with stress in adulthood.

Supported by a grant 52/2000 from the Israel Foundation Trusties to $G . R-L$

Keywords: Early stress, Learning, Anxiety.
Early exposure to platform stress modulates the ability to cope with acute swim stress in adulthood, in the rat

Avital A., Ebril E., Jacobson S. and Richter-Levin G.

Dept. of Psychology, University of Haifa, Haifa

Recent evidence supports the hypothesis that exposure to stress during early childhood may disturb the formation of functional brain pathways, in particular of the limbic system circuits, which play a major role in emotion and learning.

We therefore set out to examine the effects of early exposure to stress on the ability to cope with stress in adulthood. Rats were subjected to stress (elevated platform stress for 30 minutes) prepuberty ( 4 weeks). Post-puberty, at the age of 12 weeks, rats were exposed to adulthood stress experience (acute swim stress), and were then tested in the open-field, the Morris water-maze and the startle-reflex test. We report that an early exposure to stress led to elevated levels of anxiety, as measured in an open-field test, but that rats that were exposed to early + late stress showed a significantly higher level of anxiety compared to. control, and to rats that were exposed only to early or to late stress. Basal startle response was also elevated. Nevertheless, the early + late stress group acquired faster the spatial learning task, though following acquisition there was no difference in spatial memory performance between the different groups.

These findings suggest that an early exposure to stress may have significant implications on the ability of rats to cope with stress in adulthood.

Supported by a grant 52/2000 from the Israel Foundation Trusties to $G$. $R-L$

Keywords: Early stress, Learning, Anxiety.

\section{Maternal behavior in a genetically selected animal model of \\ depression ${ }^{1}$ Avnon Y.L. ${ }^{1}$ Yadid G. ${ }^{2}$, Shayit M. ${ }^{1}$ and Weller A. ${ }^{1}$ \\ Dept. of Psychology ${ }^{\prime}$, Faculty of Life Sciences ${ }^{2}$, Bar Ilan \\ University, Ramat-Gan}

The association between depression and maternal behavior found in humans was examined in the Flinders Sensitive Line (FSL), established through selective breeding of Sprague-Dawley (SD) rats. This line meets several criteria for an animal model of depression and displays anhedonia (Overstreet et al., Neurosci Biobehav Rev 17, 5[-68 [1993]). Since mammalian maternal behavior is maintained partially by the rewarding value of the infant to its mother, we hypothesized that FSL and SD mothers will show different patterns of maternal behavior. 10 rats from each line (FSL and SD), were observed with their litters in their home cages, four days/week during the first 3 postpartum weeks, twice during the light phase of the light/dark cycle (morning and afternoon). Frequency of behaviors was recorded from three "spot-checks" during each $30 \mathrm{~min}$. observation. Only afternoon observations revealed between-group differences.

Across all three weeks, while the groups did not differ in number of observations spent nursing, FSL mothers were found significantly less in contact with their pups than SD mothers. In the third postnatal week, FSL mothers, engaged in less nursing and in more resting and self-grooming. These results imply that as long as the pups are completely dependent on their mother for survival, FSL mothers provide for their basic needs, while supplying less non-nutritive contact than controls. However, as pups become slightly independent, FSL mothers prefer self-care over infant care. Maternal behavior has both stereotyped and reward-based components. Depression may selectively decrease the reward-based aspects.

Keywords: Animal models, Depression, Maternal behavior,

Reward.

\section{Combining human and mouse genetics helps unravel the} function of proteins in the inner ear

Avraham K.B., Ahituv N., Ben-David O., Brownstein Z., Dagan O., Davis-Silberman N., Gottfried I., Hertzano R., Sobe T., Vreugde S., Weiss S. and Ziv I.

Dept. of Human Genetics \& Molecular Medicine, Sackler School of Medicine, Tel Aviv University, Tel Aviv

The first and crucial step in the sensory processing of hearing, the transduction of sound into a cellular response, takes place in specialized cells that form an interface between our environments and our nervous systems. These cells in the cochlea, the mechanosensory hair cells, express a large repertoire of proteins 
that are involved in a remarkable feat of coordination. These include the unconventional myosin VI and POU transcription factor POU4F3. Both have very distinct roles in the hair cells, revealing just a portion of the complexity of the inner ear. Mutations in both genes are associated with human hearing loss. Studies of mice deficient in these critical components of the auditory system have revealed insights into inner ear pathophysiology and molecular mechanisms of deafness. We are finally able to go beyond the identification of the human deafness genes, and investigate the mutations that lead to hereditary hearing impairment on a functional level. Our work demonstrates the genomic and biological complexity of deafness, and how progress in the field has gone from gene identification to elucidation of protein function.

Keywords: Transcription factors, Myosins, Cochlea, Auditory system

Non-steroidal anti-inflammatory drugs enhance soluble amyloid precursor protein release

Avramovich Y., Amit T. and Youdim M.B.H.

The Eve Top fand USA National Parkinson Foundation Centers of Excellence for Neurodegenerative diseases, Bruce Rappaport Family Research Institute and Dept. of Pharmacology, Faculty of Medicine, Technion, Haifa

Alzheimer's brain shows a chronic inflammatory response, characterized by activated glial cells and increased expression of cytokines and complement factors surrounding amyloid deposits. Non-steroidal anti-inflammatory drugs (NSAIDs) have shown some success in $\mathrm{AD}$ treatment. We have found that the amyloid- $\beta$ $(\mathrm{A} \beta)(25-35)(100 \mu \mathrm{M})$ significantly enhanced the expression of the pro-inflammatory cytokines, interleukin -6 and tumor necrosis factor- $\alpha \quad($ TNF- $\alpha)$ mRNAs in human neuroblastoma $\mathrm{SH}-5 \mathrm{Y} 5 \mathrm{Y}$ cells. The involvement of NSAIDs in the regulation of the amyloid precursor protein (APP) processing was investigated, using cultured human SH-SY5Y neuroblastoma and rat PC12 cells. In this study two groups of drugs were tested:

(a) COX inhibitors libuprofen (Ibu), indomethacin (Indo) and nimesulide (Nim)] and (b) TNF- $\alpha$ suppressors [thalidomide (Thal) and supidimide (Sup)]. For the first time, we describe that these NSAIDs cause a dose-dependent $(0.1-10 \mu \mathrm{M})$ increase in the release of the non-amyloidogenic $\alpha$-secretase form of the soluble APP (sAPP) into the conditioned media of both cell lines. Enhanced SAPP secretion was recognized by both monoclonal antibodies 22C11, which recognizes the $\mathrm{N}$ terminus of APP and $6 \mathrm{E} 10$, which recognizes the $C$ terminus of sAPP, cleaved at the $\alpha$ site. Thus, the identified bands are assumed to be $\alpha$-secretasecleaved form of sAPP. In support of this, hydroxamic acid-based inhibitor, Ro31-9790, blocked the effect of NSAIDs drugs. For the first time we have provided evidence that NSAIDs promote the non-amyloidogenic $\alpha$-secretase APP processing, which may explain their therapeutic value in Alzheimer's disease.

Keyword: Alzheimer's disease, Non-steroid anti-inflammatory drugs, COX1 and 2 inhibitors, APP processing

Processing of the amyloid precursor protein by non-steroidal anti-inflammatory drugs involves the mitogen-activated protein kinase signaling

Avramovich Y., Amit T. and Youdim M.B.H.

The Eve Topf and USA National Parkinson Foundation Centers of Excellence for Neurodegenerative diseases, Bruce Rappaport Family Research Institute and Dept. of Pharmacology, Faculty of Medicine, Technion, Haifa

Brain inflammatory processes underlie the pathogenesis of Alzheimer disease (AD) and indeed, previous epidemiological studies have demonstrated a reduced risk of $\mathrm{AD}$ among individuals using non-steroidal anti-inflammatory drugs (NSAIDs). However, the mechanism by which these drugs might affect the patho-physiological processes of $A D$ has been unclear. Recently, we have shown that the NSAIDs nimesulide (Nim), ibuprofen (Ibu) and indomethacin (Indo) stimulated the $\alpha$-secretase processing of the amyloid precursor protein (APP) in a dose dependent manner $(1-100 \mu \mathrm{M})$. To further clarify the mechanism by which these drugs stimulate the secretion of the soluble fragment of APP (SAPP), the signaling pathway involved in the regulation of SAPP release was investigated. Using several signal-transduction inhibitors, it was shown that protein kinase $C$ (PKC)-, mitogen- activated protein (MAPK)- and tyrosine kinasedependent pathways mediate the effect of these drugs on SAPP secretion. Furthermore, the direct activation of MAPK was examined in rat PC12 and human neuroblastoma SH-SY5Y cells.
Western blot analysis using a phosphospecific MAPK antibody, revealed a time $(1-15 \mathrm{~min})$ and concentration $(1-100 \mu \mathrm{M})$ dependent increase in MAPK phosphorylation in cells stimulated with Nim, Ibu and Indo. The extra cellular signal-regulated kinase (ERK) inhibitors PD98059 or U0126 suppressed the drugs' induced MAPK activation. In addition, the specific PKC- inhibitor GF 109203X decreased the effect of Nim on MAPK activation, which suggests its dependence on PKC activity. The results demonstrate that these NSAIDs regulate the processing of APP via PKC-, MAPK- and tyrosine kinase- dependent pathways and could be therapeutically important for $\mathrm{AD}$ treatment

Keywords: Alzheimer's disease, Non-steroid anti-inflammatory drugs, APP, Signal transduction

Differential effect of protein synthesis inhibition in the cortex and in the amygdala on experimental extinction of conditioned taste aversions in the rat

Bahar A., Berman D.E., Dudai Y., Hazvi S., Samuel A. and Stehberg J.

Dept. of Neurobiology, Weizmann Institute of Science, Rehovot 76100

The insular cortex (IC), which contains the central taste area, and the amygdala subserve the acquisition, consolidation and retention of conditioned taste aversion (CTA) memory in the rat. The consolidation of long-term CTA memory is blocked by the local microinfusion into the IC or into the central nucleus of the amygdala $(\mathrm{CeA})$ of the protein synthesis inhibitor anisomycin immediately before training. We have recently reported that microinfusion of anisomycin into the IC immediately before or after the first retrieval test in an experimental extinction protocol blocks the extinction of CTA (Berman \& Dudai, Science 291: 2417 [2001]). This corroborates the notion that experimental extinction of CTA is not forgetting, but relearning of a new association of the conditioned taste with the absence of malaise. We now report that a similar treatment in the CeA does not block extinction. However, extinction of CTA is affected when the protein synthesis inhibitor is microinjected into the basolateral nucleus of the amygdala (BLA). Our results indicate that the effect of protein synthesis inhibition on the fate of the trace after retrieval is region dependent, and that the IC, and different nuclei within the amygdala contribute differently to the experimental extinction of the CTA trace

Supported by The Dominic Center for Higher Brain Function and The Reich Foundation and the Volkswagen Foundation. Keywords: Taste, Extinction, Protein synthesis, Learning, Rat

\section{Perception and cognitive abilities}

Banai K. ${ }^{1}$ and Ahissar M.

Interdisciplinary Center for Neural Computation, Hebrew University of Jerusalm, Israel; ${ }^{2}$ Dept. of Psychology, Hebrew University of Jerusalm, Jerusalem 91905

To what extent does impaired perceptual processing constrain cognitive abilities? Moderate correlations between various perceptual measures and intelligence have been reported anecototally. These correlations were attributed to a general perceptual/biological factor such as speed or efficiency of information processing in the nervous system. We now asked whether different perceptual tasks indeed share the same cognitive factor. To test this we administered a battery of auditory and visual perceptual tasks and standard cognitive tasks (e.g. Raven Standard Progressive Matrices) to a group of 60 participants. We found significant correlations between scores in Raven's Matrices and discrimination thresholds for auditory frequency, duration and intensity and for visual flicker detection tasks $\left(\mathrm{r}_{\text {Spearman }}=.41, .31\right.$, .36 and .36 respectively), consistent with previous reports. Surprisingly, however, there was almost no correlation between scores in the different perceptual tasks although each of them was correlated with cognitive abilities. Their contribution to the prediction of cognitive scores was almost additive. Together, auditory frequency and duration discrimination and visual flicker detection predict more than $40 \%$ of the variance in Ravens' Matrices scores. We conclude that performance in different perceptual tasks might limit different, independent, components of non-verbal intelligence. Assessing an individual's perceptual profile may thus be a useful tool in exploring his/her different cognitive skills.

Keywords: Perception, Intelligence, Cognition, Auditory processing. 
Neuroprotection by PRS-211,095: functional and morphological effects in transient MCA occlusion in rats Bar-Joseph A., Lavie V., Berckovitch Y., Weksler A., Grienstein Y. and Fink G.

Pharmos Ltd, Kiryat Weizmann, Rehovot 76326

Dexanabinol is a non-psychotropic cannabinoid, which acts as a noncompetitive NMDA receptor antagonist, and has anti-oxidant and anti-inflammatory activities. It was shown to be neuroprotective in brain ischemia and traumatic brain injury models. PRS-211,095 is a novel analog of Dexanabinol with binding affinity for the NMDA receptor and ability to inhibit cyclooxygenase- 2 about 5 and 2 times greater, respectively, than Dexanabinol. The aim of the present study was to determine whether PRS-211,095 has long-term beneficial effects on functional outcome and on infarct volume following focal brain ischemia. The middle cerebral artery (MCA) of Sprague Dawley rats was occluded for 120 minutes by intraluminal suture. PRS$211,095(0.5,2.5,5$ or $10 \mathrm{mg} / \mathrm{kg}$ IV) and its vehicle were administered at the end of the ischemic insult. The neuroprotective efficacy of the compound was evaluated by the "staircase test" and infarct volume. Rats were trained for 1 week before the insult, twice a day for 15 minutes. Thereafter, rats were tested daily for 23 weeks. At the end of the test period brains were serially sectioned and stained with $\mathrm{H} \& \mathrm{E}$. Infarct volumes were evaluated by computerized image analysis. A dose related improvement in performance in the staircase test on the contralateral side was seen with PRS-211,095 (40-80\% compared with vehicle, $\mathrm{p}<0.05$ at 0.5 , 5 and $10 \mathrm{mg} / \mathrm{kg}$ ). Infarct volume was also reduced with PRS$211,095(35 \%$ at $5 \mathrm{mg} / \mathrm{kg})$. Thus, PRS-211,095 induces functional as well as morphological neuroprotection following transient MCAo in rats.

Keywords: Ischemia, Neuroprotection, Stroke, Staircase test.

\section{Recycling implicit visual information to new composite percepts \\ Barlasov A. and Hochstein S. \\ Dept. of Neurobiology, and Neural Computation Center, Hebrew University, Jerusalem 9,1904}

As already suggested by Rock \& Linnett (Perception 22: 61-76, 1993), under certain conditions there may be a difference between the retinal image and the explicitly perceived image. We now ask what are the implications of the presence of an implicit retinal image. For example, can the implicit retinal image alone be used by the visual system to produce a new conscious percept? Since the presence of the implicit retinal image may be tested by an afterimage, we study the use of this afterimage to produce new explicit percepts. Subjects looked at a moving fixation point on a computer monitor. Bright image-components were flashed in appropriate locations relative to the moving fixation point and/or the fixed screen. Generally, the conscious percept combines the components relative to their absolute position on the screen. The retinal image, on the other hand, is always relative to the fixed or moving fixation point. We found that when the subjects viewed the afterimages, they were able to form new composite percepts combining the components anew. The components were now explicitly "rearranged" according to their retinal positions, alone. Significantly, the new percepts also included illusory forms based on the new arrangements. These findings imply that the visual system separates between information implicitly and explicitly available. Furthermore, the system may "rewind" and "replay" the perceptual process with new conditions, returning to the implicit information at a later point and re-integrating it to new explicit percepts. This process may have important implications for normal vision as well.

Keywords: Vision, Integration, Afterimage, Illusory contours

Effects of preemptive analgesia on immune response in the postoperative period

Beilin B. ${ }^{1}$, Bessler H. ${ }^{2}$, Mordashov B. ${ }^{1}$, Smirnov G. ${ }^{1}$ and Shavit Y. ${ }^{3}$ Dept. of Anesthesiology, Research institute ${ }^{2}$, Rabin Medical Center, Golda-Hasharon Campus, Petah Tiqva $49372 ;{ }^{3}$ Dept. of Psychology, Mount Scopus, Hebrew University, Jerusalem 91905

Pain may contribute to the immune suppression observed in the postoperative period. Several perioperative pain management techniques are in common use, including 1.-Patient controlled epidural analgesia (PCEA), based on a smaller dose of opiates (known to have immunosuppressive effects), in combination with local anesthetics (known to have anti-inflammatory properties); and 2.-Preemptive analgesia known to reduce postoperative pain and opiate consumption, and believed to prevent central sensitization caused by incisional and inflammatory injuries. We compared the effects of these pain management techniques on immune alterations in the postoperative period.

Patients were females undergoing trans-abdominal hysterectomy under general anesthesia, and were randomly assigned to one of two groups: 1.-Preemptive+PCEA - Epidural preoperative $(20$ min before surgery): mixture of $12 \mathrm{ml}$ bupivacaine $(0.5 \%)+$ fentanyl $(50-100 \mu \mathrm{g})$. Postoperative: mixture of bupivacaine $(0.1 \%)+$ fentanyl $(2 \mu \mathrm{g} / \mathrm{ml})$. Background continuous infusion $(6$ $\mathrm{ml} / \mathrm{hr}$ ) + per demand ( $3 \mathrm{ml}$ per press), lock-out time $10 \mathrm{~min}$. 2.PCEA - Preoperative: epidural catheter with test dose only. Postoperative: as described above

Patients of the "Preemptive+PCEA" group exhibited lower pain scores, while used the same amount of analgesic mixture. In the "Preemptive+PCEA" group, levels of proinflammatory cytokines were significantly less elevated, while levels of IL-2 were significantly less suppressed, in the postoperative period, compared with the "PCEA" group. It has been suggested that preemptive epidural block attenuates the surgery-associated neurogenic inflammation and central hyperexcitability, and presumably in this way decreases the release of proinflammatory cytokines and reduces the level of postoperative pain.

Keywords: Postoperative pain, Immunity, Cytokines, Opiates, Inflammation

Mouse forebrain serpin levels in acute experimental autoimmune encephalomyelitis (EAE)

Beilin O. ${ }^{1}$, Korczyn A.D. ${ }^{1,2}$, Gurwitz D. ${ }^{3}$, Karussis D.M. ${ }^{4}$

Ferdman $\mathbf{R}^{1}$, Mizrachi-Kol R. ${ }^{4}$ and Chapman J.,2

Depts of Physiology \& Pharmacology ${ }^{2}$, Neurology ${ }^{2}$, Genetics ${ }^{3}$, Sackler Faculty of Medicine, Tel Aviv University, Tel Aviv; ${ }^{4}$ Dept of Neurology, Hadassah Medical Center, Jerusalem

Objectives: To measure forebrain serpin levels and thrombin inhibition during acute EAE.

Methods: Severe acute EAE was induced by immunizing 8 weeks old SJL mice with spinal cord homogenates $(n=8)$. Control mice were injected with adjuvant alone $(n=9)$. On day 12 postimmunization the brains were excised, homogenized and centrifuged to separate soluble components. Inhibition of exogenous thrombin activity was measured with a labeled peptide substrate. The serpins, protein nexin I (PNI) and antithrombin III (ATIII) were measured by western blot.

Results: Soluble factors in the brain homogenate inhibited exogenous thrombin activity. In forebrain homogenates from acute EAE mice a $50 \%$ increase in thrombin inhibitory activity was observed $(p<0.05$, $t$-test). Levels of forebrain PNI and ATIII were both increased by about $50 \%$.

Conclusions: Thrombin inhibition is significantly increased in EAE mice forebrains. Our observations suggest that forebrain expression of both serine proteases and serpins might be elevated during acute EAE. The exact role of these changes in EAE remains to be established.

Keywords: EAE; Thrombin; Protein nexin I; Anti thrombin III.

\section{Melatonin promotes sleep-like state in gerbil Psammomys} Obessus

Ben-Aharon G. ${ }^{1}$, Haim A. ${ }^{2}$ and Zisapel N. ${ }^{1}$

Dept. of Neurobiology, Faculty of Life Sciences, Tel Aviv University, Tel Aviv, ${ }^{2}$ Dept. of Biology, University of Haifa at Oranim, Kiryat Tivon

The sleep promoting effect of the pineal hormone melatonin in human is known for decades. However, the mechanisms of this phenomenon remain obscure, mainly due to lack of a simple, genetically tractable, animal model that will, like human, be diurnal active.

We now report that melatonin promotes sleep-like state in a diurnal gerbil, Psammomys obessus, essentially similar to the effect induced by a conventional hypnotic, sodium pentobarbital $(0.05 \mathrm{ml} /$ animal). Furthermore, while exogenous melatonin injected in daytime $(2.5 \mathrm{mg} / \mathrm{kg}$ b.w. i.p.) induced sleep-like effect on mature psammomys (120-170gr b.w.), there was no effect on young psammomys (60-80gr) - a group characterized by high levels of endogenous melatonin, also during daytime. This data is compatible with previous findings showing $\mathrm{AMRI}$ response of the 
brain to melatonin injection $(50 \mu \mathrm{gr} / \mathrm{kg}$ b.w. i.v.) at specific brain regions of the adult psammomys brain and a different response in the young age group. This is the first demonstration of melatonin influence both on activity and brain response on a diurnal rodent and may provide an efficient animal model for further research of sleep and sleep regulation.

Keywords: Melatonin, Sleep, Activity, fMRI, Psammomys Obessus.

\section{Studies of white matter maturation of the normal brain using high $b$ value diffusion weighted imaging \\ Ben-Bashat D. . , Ben Sira L. , Graif M. and Assaf Y. The Wohl Institute for Advanced Imaging, Tel Aviv Sourasky Medical Center, Tel Aviv, 64239}

Brain development includes many aspects such as development of sulci, maturation of brain chemistry, changes in free water motion, myelination etc. Myelination can be studied by different MR techniques: $T_{1}, T_{2}$, magnetization transfer, diffusion weighted imaging (DWI) and spectroscopy. Although, the visual appearance of white matter, both on $T_{1}$ and $T_{2}$ weighted images seems to be unchanged after the age of about two years, measurements of relaxation times do show decrease of $T_{1}$ and $T_{2}$ that continues into adolescence. DWI studies showed a reduction of $\mathrm{ADC}$ with brain maturation associates with myelination. The ADC changes reach adult's values within the first 6 months. Diffusion tensor imaging shows an increase in the diffusion anisotropy from newborn to about 6 years of age.

Diffusion imaging with high $b$ value enables more specific detection of intra-axonal water. Therefore, this method is very sensitive to even minor changes of either the axon or the myelin around it. Previous studies showed that high $b$ value $q$ space analysis diffusion images are very sensitive to white matter degeneration (Assaf et al, MRM, [2001], in press). In addition, animal studies showed that this technique enable the following of myelination with high accuracy and sensitivity (Assaf et al. MRM 44: 713-722 [2000]). High b value DWI was used to study normal maturation of myelin in 22 children and adults form 3 mo to 25 yo. Region of interest analysis show changes both in probability and displacement, which continue up to adolescence. Myelination develops at different rates in different regions of the brain with the corpus callosum and the internal capsule to be the first. This method was found to be very sensitive to white matter maturation, both in the normal developed brain and in various pathologies. Keywords: MRI, Diffusion, Brain maturation, Myelination, Development

\section{Neural-population account for serial memory strategies} Ben Dayan D.D. ${ }^{1}$ and Amit D.J. ${ }^{1,2}$

${ }_{1}^{1}$ Racah Institute of Physics, Hebrew University, Jerusalem, Israel; ${ }^{2}$ Dep. of Physics, University of Rome "La Sapienza", Rome, Italy We present a framework-model, at the level of neural population rates (mean-field theory), to account for the psychophysics of monkeys performing delayed sequence recall task (Orlov et al, Nature 404:77-80 [2000]). Monkeys are presented a sample sequence of 3 images (triplet), separated by delays, then a test multi-image, containing all three + a distractor image from another triplet. Task: touch the 3 images in their previous order avoiding the distractor. The proposed model is predictive and serves as a guide for neurophysiology related to the task.

The model is based on the interaction of 3 neural modules: Image resolution, Category, Working Memory. The first and third layers have similar structure, expressing selective visual responses to the images, and are mutually connected selectively. The category module is sensitive to the ordinal position of the learned stimuli (perception or expectation of an event in a sequence). During the multi-image test, the 4 images appear in the image module in low resolution, and the choice image is focused on by selective attention. Additional signals from: 1. Category module; 2 . Working memory; 3. Intra-stimulus associations (Miyashita correlations) bring the correct image into high resolution and, after foveation, elicit motor action in the correct direction.

The simulation of the model, in different variations on the task, shows graphically how the complex set of interactions, learned during training, is able to express the required signals. It also provides a natural account for "transfer" upon category reshuffling.

Keywords: Categorization, Working memory, Multiple attractors, Learning transfer.
Prevention of 6-hydroxydopamine induced dopaminergic neurotoxicity by VK-28, a brain penetrable iron chelator

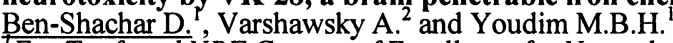
${ }^{7}$ Eve Topf and NPF Centers of Excellence for Neurodegenerative Diseases Research and Dept. of Pharmacology, Rappaport Faculty of Medicine, Technion, Haifa. ${ }^{2}$ Weizzman Institute of Sceince, Rehovot 76100

In Parkinson's disease (PD) and its animal models there is an onset of oxidative stress, where ionic iron (chetable) has a pivotal role in this and dopaminergic neurodegeneration processes. Previously we reported that desferal, a non brain permeable iron chelator, when injected intraventricular (IVC), protects against 6hydroxydopamine (6-OHDA) neurotoxicity. We examined the neuroprotective activity of our newly developed brain permeable iron chelator, VK-28, in the rat 6-OHDA model of PD. IVC

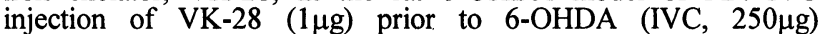
completely prevented the degeneration of rat striatal dopamine neurons as measured by dopamine, DOPAC , HVA and turn over of dopamine. This suggests that there is no interaction of VK-28 with 6-OHDA. Chronic systemic treatment $(1 \mathrm{mg} / \mathrm{kg}$ or $5 \mathrm{mg} / \mathrm{kg} /$ day for 7-10 days) of rats with VK-28 also induced neuroprotection against IVC injected 6-OHDA. A greater $(75 \%)$ neuroprotection was obtained at the higher dose of $\mathrm{VK}-28$ and striatal dopamine turnover (DOPAC+HVA/dopamine) was similar to control groups. Neither ICV or systemic VK-28 treatment altered striatal serotonin or noradrenaline, suggesting thatVK-28 has no effect on these neurons. Furthermore the body weight loss normally observed with 6-OHDA is prevented by VK-28. We have previously suggested that similar to use of copper chelator, D-penicillamine, in Wilson's disease, iron chelators such as VK28 may be employed in treatment of PD and other neurodegenerative diseases where iron accumulates at the site of neurodegeneration

Keywords: Iron, Iron chelator, Parkinson's disease, Neuroprotection.

GABA promotes neuronal tissue regeneration in hippocampal organotypic culture.

Ben-Yaakov G. and Golan $\mathrm{H}$.

Dept. of Developmental Molecular Genetics, Faculty of Health Sciences, and Zlotowski Center for Neuroscience Ben-Gurion Univ. Beer-Sheva

GABA, the main inhibitory neurotransmitter in the brain, is a natural substance that significantly control neuronal circuit activity and development. Recently, a few studies demonstrated different roles for GABA during brain development. GABAergic potentials are depolarizing at the first postnatal days. These depolarizing potentials reverse around the postnatal day 4 due to the expression of $\mathrm{K}-\mathrm{Cl}$ cotransporter; this may be reversed again following injury. In addition, GABA displays a morphogenic and chemoattractant function at the early postnatal stage. In the present study we have examined the ability of GABA to facilitate regeneration of neuronal tissues in mice hippocampal organotypic slice culture. A cut within the CA1 region of the hippocampus was made, and the rate of neuronal growth and regeneration in the injury sites were evaluated. Addition of GABA $(5-50 \mu \mathrm{M})$ to the cultured slices, immediately after the injury, was significantly facilitated the growth of neurons through the injured tissue Addition of bicuculline $(100 \mu \mathrm{M})$, specific GABA A inhibitor, together with GABA $(50 \mu \mathrm{M})$ to injured slices specifically inhibited the regeneration of the injured tissue, without affecting the viability of cells in the culture. Lower concentrations of bicuculline $(10$ and $50 \mu \mathrm{M})$ did not effectively inhibit the GABAinduced regeneration effect. The effect of GABA as supporting regrowth of neuronal tissue was blocked by suppression of glia proliferation by FUDR. These data may suggest that GABA could play a role as a growth factor in the injured tissue; this function could be mediated by the GABA A receptor and require the presence of glia cells.

Supported by Israeli Health Department, grant contract number 4841

Keywords: GABA, Regeneration, Hippocampus. 
A new approach to the temporal processing deficit in dyslexia Ben-Yehudah G. and Ahissar M. ${ }^{2}$

Depts. of Neurobiology and Psychology ${ }^{2}$, Hebrew University, Jerusalem

From the perceptual perspective, adult dyslexics can be divided to those with comprehensive deficits and those with mild deficits or none. The first have broader learning difficulties whereas the latter have very specific reading deficits (Banai \& Ahissar, Neuroscience Letters Supplement [2000]). We now asked whether this latter group of dyslexics suffers from attentional deficits. When we administered a standard attention test (Conners' Continuous Performance Test II), we found that although none of our participants would be diagnosed as having an attention disorder deficit, their scores were significantly and consistently worse than those of their intelligence-matched controls. Since this test involves different inter-stimulus intervals (ISIs), we asked whether these intervals are the source of their difficulties in the attention test.

Perceptual discriminations were thus measured under different ISIs. We first replicated previous results finding no substantial deficits in auditory frequency and intensity discriminations measured with 1-2s ISIs. With a longer ISI (5s), their frequency and intensity discriminations were both impaired (in contrast to other test groups). With very brief ISIs $(0.1-0.2 \mathrm{~s})$ their frequency discrimination was also substantially impaired. In the visual domain, their spatial frequency discrimination was similar to controls' when stimuli were presented simultaneously, but was significantly impaired when stimuli were presented sequentially.

These findings show that dyslexics have difficulties in retain-andcompare paradigms both in the visual and in the auditory modality (extending Ben-Yehudah et al., Brain 124:1381-1395 [2001]). The specificity of the deficits to atypical intervals (ISIs $\neq 1-2 s$ ) suggests an underlying deficit in temporal attention/anticipation rather than in perceptual memory.

Keywords: Dyslexia, Temporal processing, Attention, Auditory processing, Visual processing

GSK-3 and schizophrenia - relevance to the neurodevelopmental hypothesis of this disorder Bersudsky Y., Nadri C., Kozlovsky N., Belmaker RH. and Agam Stanly Center for Bipolar Research, Faculty of Health Sciences, Ben-Gurion University of the Negev, Beer-Sheva

GSK-3, a ubiquitous enzyme highly conserved during evolution, is a regulatory enzyme of an important signal transduction involved in developmental processes - the Wnt cascade. Kozlovsky et al. found $41 \%$ decrease in GSK $-3 \beta$ protein levels and $45 \%$ decrease of GSK-3 total activity ( $\alpha+\beta$ isoforms) in postmortem frontal cortex brain specimens from schizophrenic patients $v s$ comparison groups obtained from the Stanley Foundation Brain Bank. The present study addresses the question whether reduced frontal cortex GSK-3 levels in schizophrenic patients is altered in peripheral tissue and can be used as a diagnostic tool. To test that we measured fresh lymphocyte and lymphocyte-derived cell lines from schizophrenic patients and matched controls for GSK-3 $\beta$ protein levels and GSK-3 total activity. In fresh lymphocytes from schizophrenic patients there was a small $(14 \%)$ but significant reduction in GSK-3 $\beta$ levels $[75.35 \pm 57.20$ (S.D) $\mu \mathrm{U} / \mu \mathrm{g}$ protein for patients, $64.74 \pm 55.04$ (S.D) $\mu \mathrm{U} / \mu \mathrm{g}$ protein for controls, Wilcoxon, $n=18, p=0.014)$. There was no difference between patients and controls in lymphocyte GSK-3 activity. GSK-3 $\beta$ protein levels for lymphocyte-derived cell lines showed no difference between schizophrenic patients and normal controls. We conclude that the reduction in frontal cortex GSK-3 $\beta$ protein levels is reflected in fresh lymphocytes of schizophrenic patients. However, it is much smaller and could not be used as a diagnostic marker.

Keywords: Glycogen synthase kinase-3, Schizophrenia

Lymphocyte, neurodevelopment
Serotonin suppresses inhibitory $\mathbf{G A B A}_{\mathbf{A}} \mathbf{R}$ - mediated transmission in developing layer 4 of mouse barrel cortex Binshtok A.M. Fleidervish I.A. and Gutnick M.J. Koret School of Veterinary Medicine, The Hebrew University of Jerusalem, Rehovot 76100

During the first two postnatal weeks, as thalamocortical (TC) axons grow into S1 cortex, arborize, form synapses in Layer 4 and orchestrate segregation of neurons into discrete "barrels", TC cells transiently express the serotonin transporters responsible for 5-HT uptake from the extracellular space and concentration in synaptic vesicles. Since these cells are not themselves serotonergic, it has been suggest that they use 5-HT as a "borrowed" neurotransmitter. Using patch clamp techniques in tangential slices that primarily include layer 4 barrels, we have examined the postnatal development of synapses in layer 4 and have studied how 5-HT affects these synapses during development. In slices from P5-P13 mice, puff application of 5-HT induced complete and reversible blockade of stimulus-elicited GABAA-R mediated synaptic events. The effect was due to activation of presynaptic receptors, since responses elicited by direct GABA application were not affected. 5-HT did not have this effect on IPSCs in older animals $(P>18)$, and it had no significant influence on glutamatergic transmission at any age. Since there is evidence that immature neocortical neurons may have depolarizing resting potential, and that GABA may act as an excitatory neurotransmitter in developing neuronal circuits, we measured $V_{\text {rest }}$ and $\mathrm{Cl}^{-}$ equilibrium potential in unpenetrated layer 4 cells from cellattached recordings of single $\mathrm{K}^{+}$and $\mathrm{Cl}^{-}$channels. From age P5, layer 4 cells were well-polarized, and $\mathrm{Cl}^{-}$currents reversed near $\mathrm{V}_{\text {rest }}$. We conclude, therefore, that GABAergic transmission is "shunting" and inhibitory during the period of barrel development. It seems possible that during the time window when 5-HT becomes concentrated in presynaptic TC terminals, it is coreleased from these terminals along with glutamate, and that afferent activity thereby causes local disinhibition and consequent emergent bursts of synchronous cortical discharge. Such a mechanism could play a critical role in circuit formation

Supported by grant from the Israel Science Foundation

Keywords: Barrel cortex; Development; Serotonin; IPSCs

Posterior parietal (IPL) role in primate hand reaching to target behavior in the extrapersonal space (EPS) - a neurophysiological study in monkeys

Blum B.

Dept. of Physiology and Pharmacology, Sackler School of

Medicine Tel Aviv University, Tel Aviv 69978

Lesions in primate IPL cause hand-misreaching, definable as a dysfunction constrained to the perceptual/ dyspraxic, command/ motor aspects excluded. Hence the question was raised of IPL normal function in encoding integrated sensory spatial information for action schema in the EPS. Single neurone microelectrode recording was taken from awake monkey homologous area 7. Hand reaching to target (HR)-related single cell responses and also neuronal responses to visual, tactile, auditory, proprioceptive stimuli were obtained. Noteworthy are special characteristics defined: 1) At IPL's PG region same cell encoding monkey's hand reaching by stimulus-bound firing increment or decrement also shows response to simple visual or tactile stimulus. 2) HR related kinesthetic neurones with no visual response. 3) Neurones responding to visual or to another of the sensory modalities with no change in firing on HR. 4) Tactile cells at IPL's PF region showing response to touch at "target" i.e at behaviorally reward highlighted EPS location but not at "no target". 5) This IPL responsiveness could be 'learnt' by behavior experience (Blum, 1989). 6) Encoding interest/ reward-related events in the EPS including specificity for movement in that space of trainer's rewarding hand towards or away from monkey, or both directionsencoding EPS-inwards or EPS-outwards movement, thus demarcating monkey's EPS. Area 7 multiduty neurone distinct category response- same cell encoding with or without direction specificity different phases in monkey's and trainer's hand reach movement in EPS with characteristics that may be regarded components of neuronal domain cooperativity relative to high order manipulative behavior in the EPS.

Keywords: Infraparietal cortex; Hand reach; Extrapersonal space; Neurone learning response 
A novel, long-N-terminus isoform of $\mathrm{L}$-type $\mathrm{Ca}^{2+}$ channel found by screening the human genome sequence Blumenstein Y. ${ }^{1}$, Kanevsky N. ${ }^{1}$, Sahar G. ${ }^{2}$, Ivanina T. ${ }^{1}$ and Dascal N.

${ }^{I}$ Dept. of Physiology and Pharmacology, Sackler School of Medicine, Tel Aviv University; ${ }^{2}$ Thoracis and Cardiovascular Surgery, Rabin Medical Center, Petach Tiqva

The main subunit $\left(\alpha_{1 C}\right)$ of rat cardiac/neuronal L-type voltage dependent $\mathrm{Ca}^{2+}$ channel has two N-terminal (NT) isoforms, long and short, with unique initial segments of 46 and 16 amino-acids, respectively, followed by 108 fully conserved amino acids. Only a short-NT isoform has been found in humans, and only a long-NT isoform in rabbit. The initial segment of the rabbit long-NT plays an important role in channel gating and regulation by protein kinase $\mathrm{C}(\mathrm{PKC})$. Since human $\mathrm{Ca}^{2+}$ channels are regulated by PKC, we suspected that a long-NT isoform of human $\alpha_{1 C}$ should exist. By screening of the human genome for homology to rabbit long-NT cDNA, we identified a highly homologous stretch (termed exon 1a) in human genomic DNA. It contains a 5'-UTR sequence and is separated from the next known exon of $\alpha_{1 c}$ (exon $1 \mathrm{~b}$; presumably alternative; codes for the initial segment of shortNT) by an $\sim 800 \mathrm{~kb}$-long exon. The predicted 46 amino acid protein sequence is highly homologous to rabbit long-NT. Reverse transcriptase PCR showed the dominant presence of RNA composed of exons 1a, 2 and 3 in human cardiac RNA, and only a weak signal for RNA containing exon $1 \mathrm{~b}$. Western blot with an antibody against the rabbit long-NT detected a $\sim 240 \mathrm{kDa}$ protein (the size expected for $\alpha_{1 \mathrm{C}}$ ) in human atrium and ventricle. Expression of cRNA of the full-length long-NT $\alpha_{1 C}$ in Xenopus oocytes directed a PKC-sensitive $\mathrm{Ca}^{2+}$ channel, whereas the shortNT was PKC-insensitive. The long-NT isoform may underlie the $\mathrm{Ca}^{2+}$ current enhancement by PKC-activating transmitters (endothelin, angiotensin, etc.) in human tissues.

Keywords: $\mathrm{Ca}^{2+}$ channel; Isoform; Protein kinase $\mathrm{C}$; Human genome

Basal ganglia and movement: a more complex relationship than expected?

Boraud T. ${ }^{1}$, Paz R. ${ }^{1,2}$, Natan C. ${ }^{1}$, Vaadia E. ${ }^{1,2}$ and Bergman H., ${ }^{T}$ Physiology Dept., Hadassah medical school, Jerusalem; ${ }^{2}$ The Interdisciplinary Center for Neural Computation, Hebrew University, Jerusalem

Cortical information is processed through several cortico-basal ganglia loops (CBL). The degree of convergence and divergence of those pathways is still a matter of debate. This question is even more critical if we take into account inter-hemispheric communication. In this study, one monkey was trained with an unimanual/bimanual center-out task where instruction, movement and reward were clearly separated in time. We performed multielectrode recordings of the globus pallidus (GP), the internal part of which is considered as the main output structure of the basal ganglia, and of the motor cortical area during the task. Our goals were: i) to determine the responses of GP neurons to all the events in a behavioral motor task (instructions, movement and reward), ii) to determine the related timing of cortical and GP neurons during the task, iii) to determine the role played by basal ganglia in bimanual coordination. We recorded $116 \mathrm{GPe}$ and GPi cells. $62.1 \%$ displayed a response. Out of these task-related neurons, $27.8 \%$ responded to the instructions, $63.9 \%$ responded to the movement onset and $22.2 \%$ responded to reward delivery. More interestingly, $27.8 \%$ of task related neurons responded to 2 or more events. Finally, $34.7 \%$ of these task-related cells display different responses for unimanual and bimanual movements. We analyzed also the polarity of neuronal responses as well as the time relation between responding onset of GP and cortical neurons. Our data i) support CBL convergence hypothesis, ii) evidenced computation of bimanual information through the CBL. Keywords: Electrophysiology, Milti-electrode recording, Behavioral task
Herpes simplex virus type-1 latency associated gene effect upon apoptosis ex vivo Braun E. ${ }^{1}$, Mador N. ${ }^{1}$, Panet A. ${ }^{2}$ and Steiner I. ${ }^{1}$ Laboratory of Neurovirology, Dept. of Neurology, Hadassah University Hospital, Jerusalem $91120 ;{ }^{2}$ The Hebrew University,

Hadassah Medical School, Jerusalem 91120

Following primary infection, herpes simplex virus type 1 (HSV-1) persists in the peripheral nervous system in a latent state and can undergo periodic reactivations.

The only gene that is expressed during that period is the latency associated transcripts (LAT) gene. Intensive effort has been directed to reveal the role of this gene during latency and reactivation. Induction of apoptosis is critical in controlling productive and latent viral infection. Inhibition of the process could be an important mechanism for viral survival. Indeed, several viruses encode genes that interfere with apoptosis. We have postulated that the LAT gene may function as an antiapoptotic gene during latency. An earlier report (Perng G.C et al. Science 887:1500, [2000]) showed that LAT promotes neuronal survival in a neuronal cell line. We set forth to examine LAT function during apoptosis in a model that resembles the in vivo state. A transgenic mouse containing the coding sequence of the LATs under control of cytomegalovirus promoter was generated. Mouse embryonic fibroblasts (MEFs) were cultured and found to express the LAT gene. At present the ability of these cells to withstand apoptotic triggers is being examine and compared to non transgenic MEFs. Results will be presented.

Keywords: Herpes, Latency, Nervous system, Apoptosis

Parental separation leads to altered metabolic activation in different cortical brain areas

Braun K. and Bock J.

Dept. Zoology/Developmental Neurobiology, Otto von Guericke University, 39008 Magdeburg, Germany

While the basic wiring of the mammalian central nervous system and its functional pathways is genetically determined, its fine tuning throughout different phases of postnatal development is highly experience dependent. Normal brain development requires the precise interactions of environmental signals with genes and molecular signaling cascades that drive cellular differentiation and circuit formation. Early deprivation and traumatic experiences can interfere with these events, resulting in under- or maldeveloped brain circuits, which cause enduring behavioral and cognitive impairment. In order to identify those brain regions, whose functional development may be affected by juvenile traumatic experience we applied the ${ }^{14} \mathrm{C}-2$-fluorodesoxyglucose (2FDG)technique in two week old Octodon degus pups. Metabolic activation, densitometrically measured as 2FDG uptake, was compared between four experimental groups: i) pups, that were reared undisturbed with their parents, ii) pups, that were separated from their parents for 1 hour together with their littermates, iii) pups, that were separated from their parents and kept individually for 1 hour, iv) pups, that were separated from their parents and kept individually for 1 hour and, in addition, were exposed to maternal calls.

Compared to animals from group i) animals of the three separation groups (ii, iii, iv) revealed significantly reduced metabolic activity in the prefrontal cortex areas ACd (anterior cingulate cortex) and PrCm (precentral medial cortex) and also in the posterior cingulate cortex (Cg). Furthermore, individually isolated animals (group iii) showed lower metabolic activation in the $\mathrm{ACd}, \mathrm{PrCm}$ and $\mathrm{Cg}$ than animals isolated together with their littermates (group ii). Pups stimulated with the maternal call (group iv) showed a slightly but significantly increased metabolic activity in the $\mathrm{PrCm}$ and $\mathrm{Cg}$ compared to individually isolated animals without additional acoustic stimulation (group iii). No differences in 2-FDG uptake during separation were found in the amygdala. However, stimulation with the maternal call (group iv) significantly reduced 2-FDG uptake in the basomedial and lateral amygdala compared to the other three groups.

Our results indicate that early stressful situations lead to alterations of metabolic activity in distinct cortical areas. Since neuronal activation regulates the development of synapses during postnatal brain maturation, the stress-induced alterations of metabolism may interfere with the refinement of limbic connectivity patterns, resulting in altered processing of emotional stimuli in adulthood.

Supported by the state of Saxony-Anhalt LSA3006A/0088H and the Volkswagenstiftung

Keywords: Stress, Limbic system, Prefrontal cortex, Affective disorders 
Complex form discrimination by area 18 cells of the cat. Bregman T. and Yinon U.

Physiological Laboratory, Goldschleger Eye Research Institute, Tel-Aviv University Faculty of Medicine, Sheba Medical Center, Tel-Hashomer 52621

Given that cells in visual cortical area 18 in cats are able to encode information of complex shapes (Redies et al., Exp. Brain Res., 5: 469 [1986]; Hegde and Van Essen, J. Neurosci., 20, 0: RC 61 [2000]), we have studied their capability to encode the complex forms - animate contours (AC). AC (cat's body contours), various degraded and scrambled AC, ellipses and lines were presented monocularly and repeatedly to the same eye within the cell's receptive field. Each contour was presented several times. 82 complex cells in area 18 of 11 paralyzed and anesthetized cats were characterized. The main findings are as follows: 1) AC and lines elicited similar responses $(44 \%$ cells responded stronger to lines and $56 \%$ - to $\mathrm{AC} ; \mathrm{p}=0.31$, W-paired test); 2) the cells were able to discriminate between the whole and degraded AC: the responses to the whole $\mathrm{AC}$ were considerably stronger than to all degraded contours $(\mathrm{p}<0.01) ; 3)$ the cells differentiated in their responses scrambled contours with different element orientation $(p<0.001)$. Conclusions: The cells selective to any presented AC were not found in the area 18. Nevertheless, the results taken together point to the wide coding properties of these cells, and their involvement in the contour abstraction.

Supported by Miriam and Aaron Gutwirth Foundation

scholarship.

Keywords: Extrastriate, Object recognition, Receptive field

Antisense oligonucleotides suppressing acetylcholinestrase synthesis relieve neuromuscular weakness in experimental autoimmune myasthenia grayis

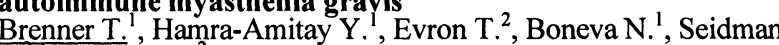
$\mathrm{S}^{2}$ and Soreq $\mathrm{H}^{2}$

Depts. of Neurology ${ }^{l}$ and Biological Chemistry ${ }^{2}$, Hadassah University Hospital and Hebrew University Medical School, Jerusalem

Myasthenia Gravis (MG) is an antibody-mediated, autoimmune neuromuscular disease in which the nicotinic acetylcholine receptor (AChR) is the major autoantigen. The typical neuromuscular weakness associated with $\mathrm{MG}$ is transiently relieved by pharmacological inhibitors of acetylcholinesterase (AChE). In our study we expanded the anticholinesterase repertoire to include 2-O-methyl-protected antisense oligonucleotides (ASON) targeted to AChE mRNA. In rats with experimental autoimmune myasthenia gravis (EAMG), Normal response to repetitive nerve stimulation and marked improvement in stamina and clinical profile was observed for at least 24 hours following a single oral administration of ASON. ASON selectively suppressed expression of the alternatively spliced readthrough or AChE-R enzyme isoform, attributing a previously unsuspected role to this splice variant in MG etiology. Elevated levels of AChE-R were detected in serum from MG patients and EAMG rats, emphasizing its involvement in these diseases. Our results show the beneficial effect of ASON treatment on muscle activity in EAMG and demonstrate a possibility of oral administration of this compound. The present findings represent the first preclinical application of antisense technology for treatment of chronic neuromuscular disorders and highlight potential advantages of gene-targeted drug therapy.

Keywords: Myasthenia Gravis, Acetyl cholinesterase, Anti-sense treatment.

Mid-hindbrain organizer determines development of dopaminergic and serotonergic neurons and regulates motor activity

Brodski C. ${ }^{1}$, Signore M. ${ }^{2}$, Vogt Weisenhorn D.M. ${ }^{1}$, Sillaber I. ${ }^{1}$, Oesterheld M. ', Acampora D. ${ }^{2,3}$, Simeone A. ${ }^{2,3}$ and Wurst W.' 'Max-Planck-Institute of Psychiatry, Kraepelinstr. 2-10, 80804Munich and GSF-Research Center, Institute for Mammalian Genetics 85758 Oberschleißheim, Germany: ${ }^{2}$ MRC Centre for Developmental Neurobiology, King's College London, Guy's Campus, New Hunts House, London SEI 9RT, UK: ${ }^{3}$ International Institute of Genetics and Biophysics, CNR, Via G. Marconi 12 80125 Naples, Italy

Midbrain dopaminergic neurons are specified rostral to the midhindbrain organizer (MHO) and hindbrain serotonergic neurons caudal to it. Here we report that in mutants in which the MHO is shifted caudally, the midbrain dopaminergic neuronal population expand to the ectopic positioned MHO and is enlarged. Complementary the size of the hindbrain serotonergic cell group is decreased. Also in mutants were the MHO is shifted rostrally, the dopaminergic and serotonergic cell populations are relocated at the new positioned MHO. However here, the size ratio of these two cell populations is changed in favour of the serotonergic neurons. In addition behavioural tests reveal that mice with a caudally shifted MHO are hyperactive and show a paradoxical calming effect after amphetamine treatment. Taken together, these results provide evidence that the position of the MHO during embryogenesis determines the location and number of midbrain dopaminergic and hindbrain serotonergic neurons in vivo. Parallels with the attention-deficit hyperactivity disorder in human suggest that an aberrant position of the MHO might be involved in the pathogenesis of this disease.

Keywords: Mid-hindbrain organizer, Dopamine, Serotonin, Attention-deficit hyperactivity disorder

\section{Differential effects of the neuropeptide proctolin on synaptic dynamics of pyloric synapses in Homarus americanus.} Brodt M. and Manor Y.

Life Sciences Department and Zlotowski Center for Neurosciences, Ben-Gurion University of the Negev, Beer-Sheva Neuronal networks are often subject to neuromodulation. Little is known about the effect of neuromodulation on short-term dynamics of synapses, and how such effects contribute to shape network output.

We chose to study this question in the pyloric circuit of the stomatogastric nervous system in the lobster Homarus americanus. In a proper modulatory environment, this circuit produces a fast $(0.5-2 \mathrm{~Hz})$ tri-phasic rhythm. The core of this circuit is a reciprocal inhibition between a pacemaker ensemble and a follower neuron. These synapses show short-term depression. The synapse from the follower to the pacemaker may be involved in frequency regulation of the pyloric rhythm.

When neuromodulatory inputs to the stomatogastric ganglion are blocked, the pyloric rhythm is abolished or slowed down significantly. We found that bath application of proctolin, an endogenous neuropeptide, restores the pyloric activity, though to frequencies smaller than control. Previous work has shown that proctolin excites a sub-set of pyloric neurons by inducing an inward current. The effect of proctolin on pyloric synapses was not examined.

We studied the temporal dynamics of the reciprocal synapses between the pacemaker and follower neurons. We found that proctolin has no effect on the synapse from the pacemaker to the follower neuron. However, proctolin potentiated the synapse from the follower to the pacemaker neuron, and decreased its frequency dependence. These results suggest that proctolin increases the synaptic efficacy between the follower and the pacemake neurons, thereby limiting the extent of excitation on the pyloric rhythm. Hence, though proctolin acts as an excitatory factor via its effects on the intrinsic properties of pyloric neurons, it may also limit the pyloric frequency to physiological bounds via its effects on the synapse from follower to pacemaker neurons

Keywords: Neuromodulation, Synaptic depression, Frequency dependence.

Learning-induced modifications in predisposition for LTP and LTD induction are correlated with changes in NMDAreceptor subunit composition

Brosh I. , Lebel D.', Quinlan E. ${ }^{2}$, Bear M. ${ }^{2}$ and Barkai E. Dept. of Morphology, Faculty of Health Sciences and Zlotowski center for Neuroscience, Ben-Gurion University, Beersheva.

${ }^{2}$ Dept. of Neuroscience, Brown University, Providence, RI, USA

We have previously shown that the susceptibility for enhancing synaptic connectivity by inducing long-term potentiation (LTP) in piriform cortex brain slices is markedly reduced after olfactory learning. Accordingly, predisposition for long-term depression (LTD) induction is increased. NMDA-mediated currents have been long thought to play a key role in inducing LTP and LTD. Moreover, experience-induced modifications in NMDA subunit composition were shown to affect LTP and LTP induction. We therefore examined whether olfactory learning is correlated with such a changes in NMDA-receptor subunit composition. 
Rats were trained in an olfactory discrimination task to distinguish between positive and negative odor cues until they demonstrated rule learning. One day after training completion, we applied immunoblot analysis to examine whether a change in the NMDAsubunit composition occurs after learning. Synaptoneurosoms prepared from the piriform cortex were probed with anti-NR2A and NR2B and NR1 polyclonal antibodies. The NR2A/NR2B ratio was significantly increased after training $(1.17 \pm 0.21, n=6$ in trained, $0.92 \pm 0.26, \mathrm{n}=8$ in naive and $0.86 \pm 0.12, \mathrm{n}=7$ in pseudo trained, $\mathrm{p}<0 . \overline{05}$ )

Application of the NMDA-receptor blocker MK-801, before each training session $(10 \mathrm{mg} / \mathrm{Kg})$, prolonged the period needed for rule learning from $6.1 \pm 1.4$ days in saline injected rats $(n=8)$ to $8.9 \pm 0.6$ days in rats injected with MK-801 $(n=8)$. However, it did not affect further acquisition of odor-memories, once rule learning was achieved.

We suggest that rule learning results with a modification in the subunit composition of the NMDA-receptor in a manner that reduces predisposition for LTP and increases predisposition for LTD induction. Concurrently, the importance of NMDA-mediated activity for further olfactory learning in decreased.

Keywords: Olfactory-learning, Piriform cortex, NMDA receptors

Towards a model for language recognition in multilingual language processing

Burteisen J.

Dept. of Linguistics, Bar Ilan University, Ramat Gan

Multilinguals identify constantly to which language words they hear or read belong, which language they use for speaking and writing. Two activation approaches have been proposed to describe how information for language identification is stored, retrieved and processed in multilingual brains. Either a language node is activated after a word has been accessed; or words are stored with language labels ("tags"). Both approaches are limited: they describe either perception, or production; and language identification is only possible at the word level. Experimental evidence, however, supports the claim that language can be identified at earlier stages of the perceptual process: consonant clusters can already determine the language even prior to access of the whole word.

In our activation-processing model, both multilingual perception and production will be considered. Languages are assumed to be organized in networks of features, letters, letter combinations, phonological and morphological representations, words and word combinations. All entries unique to one language are stored in language-specific networks. Overlapping areas of two or more language networks contain entries, shared across languages. In our model, a language is identified by processed activation, when the activation of one of the networks has reached a certain threshold. All indications of language are used in order to identify language quickly, accurately, and efficiently. Language-unique features or letters cause strong activation for only one system, sufficient to reach the threshold, and the language is clearly identified; the language of items from overlapping areas has to be ascertained by additional information, at another processing level.

Keywords: Language processing, Language recognition, Node, Tag

Vaccination with myelin-associated peptides or passive transfer of autologous $T$ cells specific to myelin basic protein after spinal cord injury in rats limits degeneration and cyst formation and induces sprouting

Butovsky O., Hauben E. and Schwartz $\mathrm{M}$.

Dept. of Neurobiology, Weizmann Institute of Science, Rehovot

Studies from our laboratory have shown that the spread of damage after central nervous system (CNS) injury (secondary degeneration) can be reduced by $\mathrm{T}$ cells specific to myelin selfantigens such as myelin basic protein (MBP) (Moalem et al., Nat Med 5:49-55 [1999]; Hauben et al., J Neurosci 20, 6421-6430 [2000]). In this study we examined whether this 'protective autoimmunity', obtained after passive $\mathrm{T}$ cell transfer or active vaccination with myelin antigens, is accompanied by neuronal sprouting, and if so, whether the twofold beneficial outcome of protection and regrowth is obtained at the cost of myelin loss. We show that after spinal cord injury in rats, accumulation of $T$ cells in the vicinity of the lesion site following active or passive vaccination is accompanied by a dramatic increase in MHC-Il expression on macrophages/microglia. This finding supports our earlier observation that activated anti-MBP $T$ cells induce expression of the costimulatory molecule $\mathrm{B} 7-2$, thereby possibly increasing the ability of microglia to support antigen-dependent $\mathrm{T}$ cell activation, which plays an important role in preserving neuronal tissue (Butovsky et al., FASEB $J 15,1065$ [2001]). The injured spinal cords of untreated control rats, after reaching a steady state, showed large necrotic cavities (cysts) containing macrophages with myelin inclusions. In contrast, neither cysts no phagocytic macrophages were observed in the corresponding areas in rats treated with anti-MBP T cells/ or in vaccinated rats. No sprouting was detected after injury in control rats; however the site of the lesion in vaccinated rats showed a marked increase in the density of sprouting. Our in vitro findings suggest that $\mathrm{T}$ cells can control the behavior of macrophage/microglia by altering the expression of TNF- $\alpha$ and TNF receptors on these cells.

Keywords: CNS, Spinal cord injury, Secondary degeneration, Autoimmune neuroprotection, Sprouting

Heterogeneity in the electrical behavior of olivary neurons revealed by dynamic clamp Chorev E. ${ }^{\text {. }}$, Yarom Y. ${ }^{1}$ and Manor Y. ${ }^{2}$

'Dept. for Brain Research, Life Sciences Institute, Hebrew University, Jerusalem ${ }^{2}$ Dept. of Life Sciences and Zlotowski Center for Neuroscience, Ben-Gurion University, Beer-Sheva Neurons in the inferior olivary nucleus exhibit subthreshold oscillations (STOs). These oscillations gradually appear during development, coincidental with the formation of gap junctions between olivary neurons. We are interested to study the mechanism underlying the STOs

Previously, we proposed a model to explain how STOs are generated. The key assumption of this model was that olivary neurons have a certain degree of heterogeneity in their channel densities. Depending on the combination of channel densities, an olivary neuron could be stable, spontaneously oscillating, conditionally oscillating or bistable. The model predicted that STOs could emerge when two quiescent neurons, belonging to different dynamical groups, are electrically coupled .

To examine this prediction, we artificially manipulate the combination of ionic conductances in individual neurons. With the dynamic clamp technique, we inject model leak or calcium conductances into olivary neurons. We use brain stem slices from immature rats, where gap junctions are not expressed. Depending on the amount of conductance added or subtracted from the original, "biological" level, this allows us to transform any olivary neuron into stable, spontaneous oscillator or conditional oscillator, For example, either decreasing the leak or increasing the calcium conductance evokes oscillatory activity in a stable olivary neuron

Next, with the dynamic clamp we electrically couple a biological olivary neuron to a model cell. We find that electrical coupling of a quiescent biological neuron to a quiescent model cell can produce STO-like activity. Moreover, different biological neurons require different coupling strengths when coupled to the same model neuron. These results support our hypothesis on the role of cellular heterogeneity and electrical coupling for producing STOs in the olivary nucleus.

Keywords: Inferior-olive, Coupling, Oscillations, Dynamicclamn.

A common mechanism underlies vertebrate calcium signaling and Drosophila phototransduction

Chorna-Ornan I. ${ }^{2}$ Joel-Almagor T. ${ }^{1}$, Cohen Ben-Ami H. ${ }^{1}$,

Frechter S. , Gillo B. ${ }^{1}$, Donald L.G. ${ }^{3}$, Selinger Z. ${ }^{2}$ and Minke B. ${ }^{1}$ ${ }^{\prime}$ Dept. of Physiology and Biological Chemistry. and the Kühne Minerva Center for Studies of Visual Transduction. Hebrew

University, Jerusalem 91120, Israel;. Dept. of Biochemistry and Molecular Biology ${ }^{2}$, University of Maryland School of Medicine ${ }^{3}$ Baltimore, MD 21201 USA

Drosophila phototransduction is an important model system for studies of inositol lipid signaling. Light excitation in Drosophila photoreceptors depends on phospholipase $C$, as null mutants of this enzyme do not respond to light. Surprisingly, genetic elimination of the apparently single inositol trisphosphate receptor (InsP $P_{3} \mathrm{R}$ ) of Drosophila, has no effect on phototransduction. This led to the proposal that Drosophila photoreceptors do not use the InsP $\mathrm{P}_{3}$ branch of PLC-mediated signaling for phototransduction, unlike most other inositol lipid signaling systems. To examine this hypothesis we applied the membrane-permeant Ins $\mathrm{P}_{3} \mathrm{R}$ antagonist 2-aminoethoxydiphenyl borate (2-APB) which has proved to be an important selective probe for assessing $\operatorname{InsP}_{3} \mathrm{R}$ involvement in various signaling systems. We demonstrated that $2-A P B$ is 
effective at reversibly blocking the response to light of Drosophila photoreceptors in a light-dependent manner at a concentration range similar to that effective in Xenopus oocytes and other cells. We show furthermore that 2-APB has no effect on the opening of the light sensitive channels, indicating that it operates upstream in the activation of these channels. The results indicate an important link in the coupling mechanism of vertebrate store-operated channels and Drosophila TRP channels, which may involve the $\mathrm{InsP}_{3}$ branch of the inositol lipid signaling pathway.

Keywords: Inositol lipid signaling, InsP3 receptor, 2-APB, TRP, Drosophila phototransduction.

Activation of the nuclear protein PARP-1 is involved in longterm memory formation in Aplysia

Cohen-Armon M.', Visochek L. ${ }^{1}$, Gui Z. ${ }^{2}$ and Schwartz J.H. ${ }^{2}$

${ }^{1}$ Sackler School of Medicine, Tel-Aviv University, Tel-Aviv, Israel; ${ }^{2}$ Center for Neurobiology \& Behavior, Columbia University, NYC, USA

PolyADP-ribosylation is a fast and transient modification of nuclear proteins catalyzed mainly by PARP-1. We have recently discovered that this most abundant and highly conserved nuclear protein is rapidly activated in electrically stimulated rat brain cortical neurons via a fast inositol 1,4,5,-trisphosphate $\left(\mathrm{IP}_{3}\right)-\mathrm{Ca}^{+2}$ mobilization, releasing $\mathrm{Ca}^{+2}$ into the nucleus (Homburg et al., $J$. Cell Biol. 150:293-307 [2000]). In accordance, PARP-1 was also activated by nerve growth factors and G-protein coupled receptors stimulating PLC activation and inositol phosphates turnover. In the pleural ganglia of Aplysia, PARP-1 was exclusively activated by a serotonergic stimulation evoking long-term potentiation (LTP). PARP-1 activation was accompanied by a concomitant intranuclear $\mathrm{Ca}^{+2}$ release. PARP-1 was not activated by serotonergic stimulation inducing a short-term response nor by stimulation inducing long-term depression (LTD). These findings indicate a novel nuclear mechanism involved in LTP, presenting the first evidence for a physiological role of polyADP-ribosylation in neuronal activity.

Keywords: PolyADP-ribosylation; PLC activation; Nuclear $\mathrm{Ca}^{+2}$; LTP.

\section{Does the novel peptide NAPVISPQ have a proliferating effect on different cell types?}

Cohen I., Divinski I. and Gozes I.

Dept. of Clinical Biochemistry, Sackler School of medicine, Te Aviv University, Tel Aviv 69978

The recently discovered, Activity-Dependant Neuroprotective Protein (ADNP) contains a sequence of 8-amino-acid peptide NAPVSIPQ (NAP), exhibiting potent neuroprotection. NAP has protected neurons in culture from cell death induced by the betaamyloid peptide toxin ( $J$. Neurochem.72:1283-1293 [1999]). NAP was shown to have qualities of a beta-sheet breaker (Segal-Ruder et al., this meeting). These properties have made NAP a possible candidate for treatment in neurodegenerative disorders such as Alzheimer's disease.

The current study examined the possible effect of NAP on cell proliferation. Four cell types were examined: MCF-7, HEK293 and neuroblastoma S5Y5. Cells were seeded in 96 well dishes at a concentration of $2 \times 10^{4}$ per well in the presence of NAP at concentrations ranging from $10^{-17}$ to $10^{-9} \mathrm{M}$ and grown for $72 \mathrm{hr}$. Cell growth was then estimated by mitochodrial activity (MTS). In the examined cells, NAP did not show any effect on cell proliferation. These results together with the previous findings that NAP has neuroprotective effects suggest that NAP may be a possible therapeutic agent in neurodegenerative disorders without side effects related to cellular proliferation.

Supported: ISF, BSF, Neufeld, ISOA, Gildor Chair

Keywords: NAP, Peptide, Proliferation, ADNP.

Polyglutamine stretches as determinants of androgen receptor localization, aggregation and activity in neurons possible modulation by melatonin

Cohen M. ${ }^{1}$, Cato A.C.B. ${ }^{2}$, Lupowitz Z. ${ }^{1}$ and Zisapel N. ${ }^{1}$

Dept. of Neurobiochemistry, Faculty of Life Sciences, Tel Aviv University, Tel Aviv; ${ }^{2}$ Forschungszentrum Karlsruhe, Institute of Genetics, Karlsruhe, Germany

Proteins containing polyglutamine stretches have recently gained much scientific awareness because of their involvement in a number of inherited neuromuscular diseases. Among these proteins is the androgen receptor (AR). Expansion of CAG trinucleotide repeats encoding expanded polyglutamine stretches in the AR has been identified as a pathogenic mutation in spinal and bulbar muscular atrophy (Kennedy's disease). Aggregation of the polyglutamine stretches in the cell nucleus and sometimes cytosol has been recognized as a common feature of all CAG repeat disorders. However, a direct link of the aggregate to the disease process is still elusive and no cure exists.

The pineal hormone melatonin acts as an androgen protagonist in vivo and in vitro. Past work from this laboratory indicated that melatonin causes nuclear exclusion of AR (1) and may potentially augment or inhibit neuronal cell death in neurons bearing the extended polyglutamine AR. We have created a neuroblastomaspinal cord line stably expressing the extended polyglutamine AR and studied the effects of melatonin androgen and their combination on AR localization and cell death. The AR was localized at the cell cytoplasm.

Melatonin treatment was associated with cytoplasmic localization of the AR whereas androgen treatment was associated with nuclear localization. Melatonin caused massive aggregation of the $\mathrm{AR}$ in the cell cytoplasm. The androgen caused some nuclear retention of the AR and did not cause aggregation. Both androgen and melatonin caused extensive cell death that was more pronounced in the presence of their combination. This is the first demonstration of modulation by melatonin of the localization and aggregation of the poly-CAG repeat AR. It deserves further assessment of the role of these phenomena in neuronal cell death. Keywords: Melatonin, Androgen receptor, CAG repeats, Localization, Aggregation

Functional interactions between synaptotagmin and voltagegated calcium channels

Cohen R. and Atlas D.

Dept. of Biological Chemistry, Hebrew University, Jerusalem Neurosecretion and regulated exocytosis depend on the rapid rise of calcium concentration, due to the opening of voltage-gated calcium channels. Synaptotagmin, a calcium and phospholipid binding protein, located primarily on synaptic vesicles, has been proposed to be the $\mathrm{Ca}^{2+}$ sensor of exocytosis. In order to establish the significance of $\mathrm{Ca}^{2+}$ channel-synaptotagmin interactions to the release machinery, we used Xenopus laevis oocytes as a heterologous expression system to characterize synaptotagmin influence on calcium channel current kinetics. Two distinct and highly conserved motifs throughout synaptotagmin isoforms, were shown to affect depolarization induced-secretion. Our results demonstrate that these motifs play an essential role in the interactions of synaptotagmin with, $\mathrm{Ca}_{v} 1.2$ and $\mathrm{Ca}_{v} 2.3$, neuronal voltage-gated channels.

Keywords: Regulated exocytosis, Synaptotagmin, Voltage-gated calcium channels

Inwardly rectifying potassium channel block by external $\mathbf{M g}^{2+}$ Cohen S, and Reuveny E.

Dept. of Biological Chemistry, Weizmann Institute of Science, Rehovot 76100

Inwardly rectifying potassium channels (Kir) are involved in many physiological actions, such as setting the state of excitability of nerve and muscle, potassium secretion and insulin release. Kir channels are also known to be blocked by divalent cations such as $\mathrm{Ba}^{2+}, \mathrm{Sr}^{2+}, \mathrm{Ca}^{2+}$, and $\mathrm{Mg}^{2+}$. The former two are known to block the channel deep within the pore and latter two are known to interact with the channel at the extracellular side. $\mathrm{Mg}^{2+}$ in particular is interesting because changes in its plasma levels have been linked to various disturbances in excitable tissues. We used single channel recordings of Kir2.1 channels expressed in xenopus oocytes to test the effect of $\mathrm{Mg}^{2+}$ ions on channel conductance. Inclusion of $\mathrm{Mg} 2+$ ions in the pipette solution reduced single channel conductance with $\mathrm{IC}_{50}$ of $1.8 \mathrm{mM}$ and Hill coefficient of 1.2. In addition, we tested the ability of $\mathrm{Mg}^{2+}$ ions to affect open channel noise characteristics. Given the blood plasma concentrations of free $\mathrm{Mg}^{2+}$ ions and the potency of these ions to block Kir2.1, we suggest that small changes in blood $\mathrm{Mg}^{2+}$ concentration may have a large effect on channel function. This therefore may contribute to larger changes in cellular excitability.

Keywords: Patch-clamp, Potassium channels, Open-channel noise 
L-DOPA increases release of noradrenaline in rat frontal cortex; a microdialysis study

Dayan L. and Finberg J.P.M.

Dept of Pharmacology, Rappaport Family Faculty of Medicine, Technion, Haifa

Degeneration of the dopaminergic nigrostriatal pathway is responsible for the motor deficit in Parkinson's disease (PD), however noradrenergic and serotonergic nuclei are also affected. A double role for noradrenergic pathway degeneration in PD has been postulated, including affective and motor components. LDOPA, the main drug used in the treatment of PD, is a precursor in the synthesis of noradrenaline (NA) as well as that of dopamine (DA). In order to assess the effect of treatment with L-DOPA on NA release, rats were treated acutely with L-DOPA and carbidopa $(50 \mathrm{mg} / \mathrm{kg}$ and $25 \mathrm{mg} / \mathrm{kg} \mathrm{p.o}$, respectively), and the frontal cortex was then perfused by microdialysis in the awake animal. Treatment with L-DOPA and carbidopa had no effect on the extracellular fluid concentration of NA, but significantly raised levels of dihydroxyphenylglycol (DHPG), as well as those of the DA metabolites DOPAC and HVA. A pretreatment with the selective noradrenergic neurotoxin, DSP- $4 \quad(50 \mathrm{mg} / \mathrm{kg}$ i.p), abolished the effect on DHPG, but did not reduce the increased concentrations of the DA metabolites. When the animals were treated with desipramine (1 $\mu \mathrm{M}$ via the probe), an increment in NA concentrations was observed following L-DOPA/carbidopa treatment. The results indicate that acute treatment with L-DOPA increases release of NA in the CNS. The mechanism for this release may be that elevated extracellular levels of DA induce release of NA by an amphetamine-like effect, since NA storage sites are saturated under physiological conditions, and addition of excess precursor would not be expected to substantially increase the storage pool available for exocytotic release.

Keywords: L-DOPA, Noradrenaline, Microdialysis, DSP 4

The perception of the quality of thermal pain

Defrin R., Ohry A., Blumen N. and Urca G.

Dept. of Physical Therapy and Dept. of Physiology and

Pharmacology, Sackler School of Medicine, Tel-Aviv University, Tel Aviv 69978

Several hypotheses have been developed in the attempt to explain the neural processes underlying the determination of different pain qualities (e.g., burning, pricking, etc.), two of which are still viable today." The theory of a "labeled-line code" (specificity) maintains that pain is processed by dedicated pain pathways, and predicts that excitation of a particular nociceptor by a stimulus always elicits the same quality of pain, regardless of the stimulus energy. The alternative view is that the perception of pain is not monopolized by properties of nociceptors, rather central processes integrate sensory information including those derived from the response of nociceptors to produce the intensity and quality of sensations of which pain is one.

To test these hypotheses we conducted quantitative and qualitative somatosensory testing in spinal cord injury (SCI) subjects with complete or partial loss of non-noxious thermal sensations (either warmth, cold or both) but who still perceive pain. We found that, in skin areas lacking any thermal sensibility, high intensity warm and cold stimuli always produced a sensation of pricking pain with no thermal quality. In skin areas in which only a single thermal modality remained intact, the quality of the perceived sensation was not determined by the thermal stimulus but by the intact modality (paradoxical sensation); cold stimuli were perceived as warm in areas in which only warm sensation was preserved and vice versa.

Our results show that the quality and intensity of nociception in these patients is highly dependent on conduction in non-noxious channels. Thus, our results lend strong support to the notion that the quality of pain is determined by central integrative processes utilizing sensory information conveyed, simultaneously, by both dedicated pain pathways and non-nociceptive pathways Keywords: Thermal pain, Pain quality, Paradoxical sensations, Specificity

\section{Differential protective effects of the femtomolar acting} peptide, NAP against oxidative stress in multiple cell lines Divinski I., Cohen I., Steingart R.A. and Gozes I.

Dept. of Clinical Biochemistry, Sackler School of Medicine, Tel Aviv University, Tel Aviv 69978

Oxidative stress plays a role in many neurodegenerative disorders, e.g. Alzheimer's disease and Parkinson's disease. The recently discovered, Activity-Dependant Neuroprotective Protein (ADNP; J.B.C. 276:706-714 [2001]) contains a sequence of 8-amino-acid neuroprotective peptide NAPVSIPQ (NAP). NAP exhibits potent neuroprotection against oxidative stress in PC12 cells (Brain Res. 854: 257-262 [2000]; J. Molec. Neurosci. 15:137-145 [2000]). The present study was designed to examine the breadth of NAP ability to protect different cell types from oxidative stress. The following cell lines were examined: COS-7, MCF-7, HEK293, BJ, PC12, NIH 3T3 and neuroblastoma S5Y5. Cells were exposed to $300 \mu \mathrm{M} \mathrm{H} \mathrm{O}_{2}$ for $16 \mathrm{hr}$. in the presence or absence of NAP at concentrations ranging from $10^{-17}$ to $10^{-9} \mathrm{M}$. In the COS-7, MCF$7, \mathrm{BJ}$ and NIH 3 T3 cell lines, NAP did not produce any protective effect in all the examined concentrations. In contrast, in PC12, HEK293 and Neuroblastoma S5Y5 cell lines, NAP exhibited a protective effect. In the PC12 cell line, treatment with $300 \mu \mathrm{M}$ $\mathrm{H}_{2} \mathrm{O}_{2}$ reduced cell survival to $30 \%$ as compared to control, while addition of NAP at concentrations ranging from $10^{-17}$ to $10^{-15} \mathrm{M}$ increased cell survival to $60-70 \%$. In HEK293 and neuroblastoma S5Y5 cells, NAP exhibited lesser potency and efficacy as compared to $\mathrm{PC} 12$ cell protection. These results suggest that in contrast to PC12, HEK293 and neuoroblastoma S5Y5 that respond to NAP, COS-7, MCF-7, BJ, NIH 3T3 are not affected by NAP. This implicates that COS-7, MCF-7, BJ and NIH 3T3 may not have receptors/binding proteins that recognize NAP, suggesting specificity for NAP activity.

Supported: ISF, BSF, Neufeld, ISOA, Gildor Chair.

Keywords: NAP, ADNP, Oxidative stress, Cell lines

The influence of training and experience on neuronal graft development

Döbrössy M.D. and Dunnett S.B.

The Brain Repair Group, School of Biosciences, Cardiff University, UK

The environment, training, and experience bear upon indices of brain function and plasticity. However, it is not known to what degree these changes are mirrored in neural grafts placed within the brain. It is thought that for animals to draw functional benefits from the graft, specific training is necessary to allow them to "learn to use the transplant". To further explore this concept, rats were pre-trained on the paw-reaching task, lesioned unilaterally in the lateral dorsal striatum with quinolinic acid, and given intrastriatal suspension grafts prepared from E15 whole ganglionic eminence. The grafts were allowed to mature for 20 weeks. During the entire post-graft period half the animals participated in a daily non-specific manual dexterity task. Training induced graft plasticity was evaluated using behaviour, electrophysiology and immunohistochemistry. The results shed light on the question of functional reconnectivity of the grafts, and more importantly, whether the graft integration with the host is responsive to behavioural manipulation.

Keywords: Environment, Training, Transplantation, Rat.

Chaos in the dopaminergic system of normal and depressive

brain ${ }_{\text {Dremencov E. }}^{1}$, Nacshoni E. ${ }^{2}$, Levi D. ${ }^{3},{ }^{3}$ and Yadid G. ${ }^{1}$

${ }^{7}$ Life Sciences Faculty, Bar-Ilan University, Ramat-Gan; ${ }^{2}$ Geha

Hospital, Petach Tikva; ${ }^{3}$ Dept. of Psychology, Tel-Aviv University, Tel Aviv

Flinders sensitive line (FSL) is a rat model of depression. Our previous studies found that FSL rats show functional abnorrralities of the ventral tegmental area (VTA) - nucelus accumbens circuit and its serotonergic regulation. The aim of this work was to assess nonlinear dynamics of the DA cells in the VTA. Male Sprague-Dawley (normal) and FSL rats $(250 \mathrm{~g}$ ) were used in the experiment. The animals were anesthetized with chloral hydrate and a tungsten 0.01 " $5 \mathrm{M} \Omega$ electrode was inserted into the VTA. Single unit recording was carried out by amplitude discrimination. DA cells were recognized by their firing patterns and data series of 2000 events were recorded. Short-time correlation was analyzed by reconstruction of probability density function (PDF) of interspike intervals (ISI). Long-time correlation was analyzed by the calculation of point correlation dimension (PD2) of interspike intervals. We found that ISI of the DA cells in the VTA of FSL rats showed Gaussian PDF, while ISI of the DA cells in the VTA of normal rats showed Power Law PDF which corresponds with deterministic chaos. Additionally, FSL rats showed higher $(3.92+0.11, n=8)$ mean PD2 value than normal animals $(3.19+0.23, n=7, p<0.02)$. Thus, DA cells in the VTA of FSL rats show a higher level of randomness than in VTA of normal animals. This suggests that nonlinear analysis may be a more accurate predictor for abnormality in the dynamics of depressive behavior.

Keywords: Depression-Dopaminergic, System-Nonlinear Analysis 
The use of cDNA microarray in neuropharmacological experiments

Drigues N., Sagi Y. and Youdim M.B.H.

The Eve Topf and USA National Parkinson Foundation Centers of Excellence for Neurodegenerative diseases, Bruce Rappaport Family Research Institute and Dept. of Pharmacology, Faculty of Medicine, Technion, Haifa

cDNA microarray is a relatively fast and throughput method for simultaneously analyzing the expression of thousands of genes and it is being widely employed, especially in the field of cancer. In spite of its advantages, most of its users confront considerable difficulties in interpreting the results. In our laboratory considerable experience has been obtained with this technique, especially in the field of neuropharmacology. In order to minimize both false positive and false negative results some aspects of the pharmaco-array trial should be addressed, among them considerations of setting up the experiment, number of replicates, analysis and verification of the results. Our experience with microarray has shown that it can be a powerful technique to apply to already established models and to delineate the mechanism of the action of drugs. We strongly encourage the use of several classes of drugs when a mechanism of action of an unknown is being established. The inclusion of a negative control, a drug with similar structure but devoid of the desired pharmacological action, should be included. For example, the anti-Alzheimerantidepressant and MAO-A and -B inhibitor drug, TV3326, induces gene expression changes in the forced swimming test model of depression, similar to other known antidepressants. However its optical isomer, not a MAO inhibitor, does not. In addition, the contribution of several intra- and inter-experimental repetitions is vital for establishing the significance of gene expression. Several relevant analysis and statistic tools will be

keywonted.

development

Reference frames in reaching to visual targets

Dvorkin A.Y. ${ }^{2}$, Flash T. ${ }^{2}$, Bentin S. , , Soroker N. ${ }^{3}$ and Behrmann

$\mathrm{M}^{4}$

${ }^{\prime}$ The Interdisciplinary Center for Neural Computation, The Hebrew University, Jerusalem, Israel; ${ }^{2}$ Dept. of Computer Science and Applied Mathematics, Weizmann Institute of Science, Rehovot; Israel ' Loewinstein Rehabilitation Center, Ra'anana, Israel; "Dept. of Psychology, Carnegie Mellon University,

Reaching toward a visual target involves the transformation of visual information into appropriate motor commands. Neuropsychological and neurophysiological studies have provided evidence that the brain makes use of multiple spatial reference frames and have indicated that the parietal cortex is central to the construction of such representations.

Studies of neglect patients with discrete lesions in the parietal cortex, (usually in the right hemisphere), have been concerned with the question with respect to what coordinate frame the 'left side' is defined. Previous studies have shown that spatial position might be coded with respect to the midline of the head, trunk or even the limb.

In an attempt to identify the reference frames used to represent target position during reaching, we measured and characterized constant and variable end-point errors in a double-step target displacement paradigm, while subjects reached toward visual targets, located within the horizontal plane.

Our results showed that in pointing, the orientation of the axes of maximum variability was along the average movement direction, (this may suggest a hand-centered reference frame), while in double-step movements various orientations were found, which could suggest the effect of motion planning rather than mere execution

The initial direction of motion in healthy young volunteers was found to depend on the time difference between the second target presentation and movement onset, and included averaged/nonaveraged and direct trajectories. In contrast, the results from one stroke patient showed that averaged modified trajectories were hardly found. In addition, long pauses were observed in doublestep movements in particular when the first target appeared ipsilesionally, with respect to the trunk-midline.

Keywords: Reference frame, Reaching, Variable error
Faces do pop out: a new high-level pop out effect

Elgavi-Hershler O. and Hochstein S.

Dept. of Neurobiology, and Neural Computation Center, Hebrew University, Jerusalem, 91904

In the past, only basic features were thought to pop out from an array of distractors. More recent research has shown that highlevel concepts may pop out as well. We presented subjects with line drawings of faces, cars and houses as targets or distractors. Reaction time was independent of set size from 16 to 64 items when the target was a face and the distractors cars or houses, but increased with size when the target was a car or a house on a background of houses or cars, respectively.

The data indicate that certain important categories of objects are not detected by a relatively slow, focused conjunction search, but by a faster system that is comparable to feature search.

Although previous research has maintained that faces do not pop out from an array of distractors, our research indicates differently. The discrepancy might be traced to the use of highly schematic faces in previous research, whereas our stimuli were more realistic. The results are in accord with recent RSVP experiments that indicate that perception and categorization of high-level objects can be much faster than previously thought. Specifically, the results indicate that the visual system may be using a fast system with spread attention to find the gist of the scene, and possibly a slower system with focused attention to fill in the details. This distinction was described in the recently proposed reverse hierarchy theory as, respectively, the "vision at a glance" and "vision with scrutiny" subsystems.

Keywords: Popout, Faces, Reverse hierarchy theory

6-hydroxydopamine increases ubiquitin-conjugates and protein degradation: Implications for the pathogenic of

Parkinson's disease.

Elkon H., Melamed E. and Offen D.

Dept. of Neurology, Felsenstein Medical Research Center, Beilinson Campus and Tel Aviv University, Sackler School of Medicine, and Rabin Medical Center, Petah Tiqva 49100

One of the hallmarks of Parkinson's disease (PD) is the finding of pathological structures containing inclusions of ubiquitinated proteins in the dopaminergic neurons in the substantia nigra termed Lewy bodies. The mechanism leading to the formation of those aggregates is unclear, although it was shown that mutations in alpha-synuclein, or in the ubiquitin-related enzyme UCH-L1 might induce such protein aggregation. We therefore examined the possible role of 6-hydroxydopamine, a dopaminergic neurotoxin used in PD experimental models, in causing protein degradation and its association with the ubiquitin system. Using anti-ubiquitin antibodies we found that exposure of SH-SY $5 Y$ neuroblastoma and PC12 cell lines to 6-OHDA increased, dose dependently, the levels of free ubiquitin and ubiquitin-conjugated proteins. Furthermore, metabolic labeling with ${ }^{35}$ S-methionine, demonstrated that 6-OHDA markedly increased protein degradation as indicated by the secretion of protein metabolites to the medium. Inhibition of the proteasome assembly by the specific inhibitor MG132, attenuated the protein degradation induced by 6-OHDA and potentiated its toxicity. Administration of the antioxidant $\mathrm{N}$-acetylcysteine to the 6-OHDA-treated cells, increased cell survival and reduced protein degradation. In conclusion, our findings suggest that 6-OHDA toxicity is associated with protein degradation, which is coupled to the ubiquitin system integrity.

Keywords: Parkinson's disease, 6-hydroxydopamine (6-OHDA), Ubiquitin proteasome pathway.

Interhemispheric Negative Correlation of fMRI Signal During

Motor Task

Yeshurun $\mathrm{Y}_{.}^{2}$, Kahn I. ${ }^{4}$, Malach R. ${ }^{5}$, Fried I. ${ }^{6}$ and Hendler T. ${ }^{2}$

${ }^{I}$ Hebrew University, Jerusalem, Israel; ${ }^{2}$ Functional Imaging Laboratory, Wohl Institute for Advanced Imaging, Tel Aviv

Sourasky Medical Center, Tel Aviv, Israel; ' Sackler School of Medicine, Tel Aviv University, Tel Aviv, Israel; "Massachusetts Institute of Technology, USA; ' Weizmann Institute of Science, Reovot, Israel; ${ }^{6}$ Epilepsy and Neurosurgery Units, Tel Aviv, Sourasky Medical Center, Tel Aviv, Israel

Interhemispheric primary motor cortex neural negative correlation has been demonstrated by Transcortical Magnetic Stimulation (TMS) (Ferbert et al J Physiol 1992;453:525-546) and EEG in healthy subjects as well as in patients(Hanajima et al Clin Neurophysiol. 2001 Apr;112(4):623-6). 
Here we address this issue by using fMRI to explore the interhemispheric negative correlation by blood-oxygenation-leveldependent (BOLD) contrast during the performance of unimanual motor tasks.

Methods: Seven healthy subjects performed a unimanual visually guided finger tapping versus rest with fixation task. Images were acquired on 1.5 GE scanner. fMRI data was analyzed using BrainVoyager 4.4 software.

Results: Contralateral activation of M1 and bilateral activation of the pre-motor cortex was observed as expected. An additional analysis showed that: 1 . There was a decrease of BOLD signal in the ipsilateral primary motor cortex (ROI) during the fingertapping task compared to the rest state. 2 . In the very same anatomical location (ROI) there was an increase of the BOLD signal during movement of the contralateral hand.

Discussion: Based on the connection between LFP and AMR signal on one hand (Logothetis et al. Nature. $2001 \mathrm{Jul}$ $12 ; 412(6843): 128-30$ ), and the relative separation of the two hemispheres' vascular supply on the other, we tend to interpret the findings as supporting interhemispheric inhibition during unimanual simple movements. We speculate that this may reflect bilateral neuronal competition.

Keywords: Interhemishperic inhibition, fMRI, Finger tapping,

Motor

Transgenic AChE exerts non-catalytic effects on hippocampal LTP via PKC modulation.

Farchi N ${ }^{1,2}$, Hochner B. ${ }^{1}$ and Soreq $\mathrm{H}^{2}$

Depts. of Neurobiology and Biological Chemistry ${ }^{2}$. The Institute of Life Sciences, The Hebrew University of Jerusalem, Jerusalem The involvement of the cholinergic system in LTP has been intensively investigated. Transgenic mice overexpressing the synaptic isoform of acetylcholinesterase (AChE-S) exhibit learning and memory impairments. Furthermore, their hippocampal LTP pattern is altered, displaying unstable immediate and late phases, which cannot be rescued by application of either physostigmine or carbachol. We therefore challenged LTP in mice transgenic for catalytically inactive AChE-S, which displayed failure pattern similar to that of AChE$\mathrm{S}$ mice. These findings suggest a non-catalytic involvement of $\mathrm{AChE}$ in the maintenance phase of LTP. Interestingly, excess of soluble AChE, produced in transgenic mice for the "readthrough" AChE isoform, AChE-R, does not alter the late phase of LTP but rather induces higher level of potentiation. Since PKC is a major component in LTP stabilization we tested the option that these alterations in LTP involve the PKC pathway. Immunoblot analysis of AChE-S and inactive AChE-S transgenic hippocampi demonstrated lower levels of PKC a significantly higher level of PKC $B I$ in AChE-R transgenic hippocampus. Moreover, chemical induction of LTP by the PKC activator, phorbol di-butyrate $(5 \mu \mathrm{M})$ lead to limited potentiation of $117 \pm 13 \%$ in AChE-S hippocampal slices compared with 160 $\pm 9 \%(P<0.04)$ in control slices. This suggests a direct correlation between LTP and PKC levels in the tested mouse lines. However, with preceding PKC activation, AChE-S slices were readily activated with tetanic stimulation to $185 \pm 11 \%$ of baseline level that long lasted with a stable potentiation. Altogether, a noncatalytic involvement of $\mathrm{AChE}$ predicts an active role in neuronal plasticity via the modulation of PKC

Keywords: Acetylcholinesterase, LTP, PKC.

Tuning curves of auditory cortex neurons under halothane anasthesia

Farkash D., Bar-Yosef O., Ulanovsky N., Ahdut L. and Nelken I. Dept. of physiology, Hebrew University - Hadassah Medical School and the Interdisciplinary Center for Neural Computation, Hebrew University, Jerusalem

Most studies of auditory cortex neurons are done under barbiturate anesthesia. Under these conditions, most neurons have purely onset responses, and have unimodal tuning curves. In our lab we work with halothane anesthesia for several years now. Anesthesia is known to strongly affect responses in auditory cortex, and this study summarizes our findings regarding responses to pure tones under halothane anesthesia.

We recorded tone responses of over 1200 neurons from the primary auditory cortex (AI) of 20 cats, anesthetized by breathing a mixture of $\mathrm{O} 2 \mathrm{~N} 2 \mathrm{O}(30 \% / 70 \%)$ with halothane $(0.2-2.0 \%$, as required). $115 \mathrm{~ms}$ - long tones were presented usually at 8-11 equally spaced levels and 45 frequencies linearly spaced along a logarithmic frequency axis over a range covering the full frequency response of the neuron.

We observed a much richer array of response types and tuning curve shapes than are found under barbiturate anesthesia. In particular, a substantial number of neurons had responses extending beyond onset, some of them showing responses lasting long after the offset of the stimulus. Neurons with all tuning curve shapes reported under barbiturate anesthesia were found, but other shapes were also found, including wide-band neurons that respond over a large frequency range. Thus, auditory cortex under halothane anesthesia shows richer physiological response types than under barbiturate anesthesia. Some of these responses resemble responses recorded from awake animals.

Keywords: Auditory cortex, Tuning curve, Frequency response area, Halothane anesthesia.

Immunocytochemical localization of $\mathbf{G A B A}_{\mathbf{A}}$ receptor subunit in crayfish neuromuscular junction

Feinstein N. ${ }^{1}$, Rashkovan G. ${ }^{\text {, }}$, Fritschy J.M. ${ }^{2}$ and Parnas I. ${ }^{1}$

The Otto Loewi Minerva Center for Cellular and Molecular Neurobiology, Dept. of Neurobiology, The Hebrew University of Jerusalem, Jerusalem, 91904, Israel; ${ }^{2}$ Institute of Pharmacology, University of Zurich, Zurich CH8057, Switzerland

The neuromuscular system of crustaceans has a remarkable similarity to mammalian CNS synapses, and as such it is a good model to study modulation of neurotransmitter release by presynaptic receptors. The crayfish muscle is innervated by a single excitatory axon releasing glutamate and a single inhibitory axon releasing GABA. The presynaptic excitatory terminal possesses $G A B A_{A}$ and $G_{A B A}$ receptors, and also receptors to glutamate, 5HT and dopamine. The presynaptic inhibitory terminal possesses glutamate and $\mathrm{GABA}_{\mathrm{B}}$ receptors (Miwa and Kawai, J.Neurophysiol. 63(1):173-180 [1990]).

In the present work we provide electron microscopy immunocytochemical results supporting the existence of GABA receptor on the inhibitory terminal. The $\mathrm{GABA}_{\mathrm{A}}$ receptor was identified by a prefixation immunostaining method using a polyclonal subunit-specific antiserum raised against an extracellular epitope on the N-terminal domain of $\mathrm{GABA}_{\mathrm{A}}$ receptor subtypes (in mammalian brain). In order to check whether the $\mathrm{GABA}_{\mathrm{A}}$ receptor staining is localized on an excitatory or inhibitory terminal it was required to eliminate one of the two axons. This was done by an intra-axonal injection of protease that caused lysis of one axon. Following such a treatment, the tissue was exposed first to a prefixation gold immunostaining of $\mathrm{GABA}_{\mathrm{A}}$ receptor and then to a postembedding gold immunostaining of GABA transmitter. These experiments showed that when the excitatory axon and terminals were eliminated, $\mathrm{GABA}_{\mathrm{A}}$ specific staining appeared on the remaining healthy inhibitory terminals. We conclude that both the excitatory and inhibitory presynaptic terminals in crustaceans posses $\mathrm{GABA}_{\mathrm{A}}$ and $\mathrm{GABA}_{\mathrm{B}}$ receptors. Keywords: $\mathrm{GABA}_{\mathrm{A}}$, Receptor, Immunogold, Localization

Mitochondrial calcium dynamics in hippocampal neurons Fendyur A. Kaiserman I. and Rahamimoff R.

Dept. of Physiology and the Bernard Katz Minerva Centre for CellBiophysics, Hebrew University-Hadassah Medical School, Jrusalem

Calcium ions play a key role in a wide variety of neuronal functions. One of the calcium regulators is the mitochondrion. In recent years, this organelle has been implicated in a large number of pathophysiological states. Hence we studied calcium dynamics in neuronal processes in the vicinity of mitochondria, using confocal microscopy.

Cultured hippocampal neurons (4-9 days old) of neonatal Sabra rats were used to study mitochondrial distribution in neuronal processes and calcium dynamics in the vicinity of these organelles. Using mitochondrial and calcium-specific fluorescent dyes (Fluo-4 and Mitotracker) we double stained hippocampal neurons and simultaneously sampled both mitochondrial location and calcium dynamics.

The following observations were made:

There is a non-homogenous distribution of mitochondria in neuronal processes.

Calcium concentration $[\mathrm{Ca}++]$ is higher at locations with high Mitotracker fluorescence.

The intra-mitochondrial calcium concentration is nonhomogenous. 
Intra-mitochondrial $[\mathrm{Ca}++]$ fluctuations were independent of calcium fluctuations in the cytosolic vicinity or of calcium fluctuations in neighboring mitochondria.

Intra-mitochondrial $[\mathrm{Ca}++]$ waves were observed

Occasionally, the juxta-mitochondrial $[\mathrm{Ca}++]$ was correlated with the mitochondrial [ $\mathrm{Ca}++]$.

These results indicate that mitochondria may serve as local regulators of intracellular calcium concentration.

Supported by the US-Israel BSF and ISF

Keywords: Calcium dynamics, Hippocampal neurons,

Mitochondria, Confocal microscopy.

\section{The influence of selective attention on pattern adaptation} Festman Y. and Ahissar M.

Dept. of Psychology, Hebrew University, Jerusalem 91905, Israel Prolonged viewing of high contrast grating causes two types of adaptation: a) an increase in the contrast threshold for detection of gratings with the same or similar spatial frequency and orientation (contrast adaptation), and b) a 3-4 $4^{\circ}$ bias in orientation judgment away from the adapted orientation (tilt aftereffect). We now asked whether these two types of adaptation are affected by the attentional mode of the viewer.

Contrast threshold and vertical judgments were measured before and after adaptation under three behavioral conditions. In all 3 conditions the observer was asked to compare between successively presented patches of gratings. In two conditions observers were asked to compare gratings' orientation and contrast, respectively, thus attending the area of the adapted stimulus, though not necessarily the relevant dimension. In a third condition they were asked to compare the size of fixation, thus focusing their attention on the central area.

We found that the magnitude of contrast adaptation did not depend on the behavioral task. Contrast thresholds were elevated by $\sim 1 \%$ in all three conditions. On the other hand, the magnitude of the tilt aftereffect was significantly smaller in the fixation condition $\left(2^{\circ}\right.$ compared with $-3.5^{\circ}$ in the other 2 conditions). Thus, lack of spatial attention decreases the tilt aftereffect but does not affect contrast adaptation

The different dependencies of contrast and orientation adaptation on the behavioral task suggest that these two types of adaptation stem from different mechanisms operating at different levels of visual processing.

Keywords: Selective attention; Spatial attention; Plasticity;

Contrast adaptation; Tilt aftereffect

\section{Physical Interaction of brain Voltage-Gated $\mathrm{K}^{+}$channels with} the exocytotic proteins in brain synaptosomes

Fili O. Bledi Y 2 , Linial M 2 , Lotan I.

Dept. of Physiology, Sackler School of Medicine, Tel-Aviv University; Dept. of Biological Chemistry, Life Science Institute,

The Hebrew University, Jerusalem

The role of voltage-gated potassium channels in neurotransmitter release by virtue of their ability to shape action potential in nerve terminals has already been estabblished. However, the possibility of a direct interaction between these channels and the synaptic release complex has never been examined.

The minimal comlex essential for synaptic release contain of the three SNARE proteins -syntaxin, VAMP and SNAP-25. Synaptotagmin is also involved in this complex, probably as a $\mathrm{Ca} 2+$ sensor. Recent studies have shown that presynaptic $\mathrm{N}$ and $\mathrm{P} / \mathrm{Q}$-type calcium channels interact directly with the complex. In previous studies we showed that the $\operatorname{Kv} \beta$ subunit interacts with syntaxin 1A. We showed that this interaction occurs in rat brain synaptosomes. In this study, we show that this interaction is not limited only to the $\mathrm{Kv} \beta$ subunit, but also to the $\mathrm{Kv} 1.1$ and to the Kv2.1 potassium channels' $\alpha$ subunits. Regarding the Kv1.1 channel, we could co-precipitate in rat brain synaptosomes syntaxin $1 \mathrm{~A}$ and synaptotagmin using antibodies against Kv1.1, and GST-fusion proteins corresponding to the cytoplasmic Nterminus of Kv1.1. In vitro binding experiments revealed that the interaction between syntaxin and the N-terminus of Kv1.1 is direct. regarding the Kv2.1 channel, we could co-precipitate syntaxin and synaptotagmin from rat brain synaptosomes using antibodies against Kv2.1. The interactions in brain synaptosomes were found to be sensitive to the physiological conditions of the synaptosomes. Overexpression of Kv2.1 in PC-12 cells caused a large increase in neurotransmitter release, triggered by depolarization with elevated concentrations of $\mathrm{KCl}$. These results may suggest that the interaction of Kv2.1 with syntaxin can affect neurotransmitter release.

Keywords: Synaptosomes; PC-12; Kv1.1; Kv2.1; SNARES
Novel apoptosis-related protein ARTS increases in discrete brain areas following nęurotoxins and injury Finberg J.P.M. ${ }^{1}$, Maor G. ${ }^{2}$, Reiter I. ${ }^{2}$, Dayan L. ${ }^{\text {, Lobova E. }{ }^{1} \text { and }}$ Larisch S

Depts. of Pharmacology $I$ and of Anatomy and Cell

Biology ${ }^{2}$, Rappaport Faculty of Medicine, Technion, Haifa:

${ }^{3}$ Pathology Dept, Rambam Medical Center, Haifa

The novel mitochondrial septin-like protein ARTS, mediates TGF$\beta$-induced apoptosis in certain peripheral cell lines such as COS and NRP-154 (Larisch et al, Nature, Cell Biol 2:915-921 [2000]). In the nervous system, however, ARTS may well play a neuroprotective, rather than a neurotoxic role, as is the case for TGF- $\beta$. Levels of ARTS in discrete areas of CNS have been studied by immunohistochemistry. In mouse, rat and man, only rare cells of substantia nigra pars compacta showed positive staining for ARTS. In rats and mouse, frequent ARTS-positive cells were seen in the granular layer of the hippocampus, and a much lower level in striatum. Additional scattered cell groups expressing ARTS were seen in cortex and thalamus, particularly piriform cortex. Following neurotoxic doses of methamphetamine and MPTP in C57-B16 mice, a profound increase in ARTS immunoreactivity was seen in granular cell layer of hippocampus, and increased numbers of ARTS-positive cells were also seen in striatum, but no increase in ARTS content was seen in substantia nigra. ARTS-positive cell bodies were seen around the site of penetration injury in cortex and sub-cortical areas of rat brain. Since the increased ARTS content was seen at short times after neurotoxin administration in mouse $(4 \mathrm{~h})$, or penetration injury in rat $(1 \mathrm{~h})$, it may be that ARTS plays a neuroprotective role in the acute response to injury. The absence of ARTS from the substantia nigra pars compacta may be a factor in the sensitivity of this area to oxidative stress and other types of challenges.

Keywords: Neuroprotection, Substantia nigra, TGF $\beta$, Neurotoxins

Somato-dendritic $\mathrm{Na}^{+}$channels cannot account for persistent $\mathrm{Na}^{+}$current in layer 5 neocortical neurons Fleidervish I.A. ${ }^{1}$, Astman N. ${ }^{2}$ and Gutnick M.J. ${ }^{1}$ Koret School of Veterinary Medicine, Hebrew University of Jerusalem, Rehovot ; ${ }^{2}$ Zlotowski Center for Neuroscience, BenGurion University of the Negev, Beer Sheva

Neocortical neurons exhibit a low-voltage-activated, slowly inactivating "persistent" $\mathrm{Na}^{+}$current $\left(\mathrm{I}_{\mathrm{Nap}}\right)$, that plays a role in neuronal excitability and synaptic integration. A prevalent view is that $I_{N a P}$ is mediated by late openings of the same channel that underlie fast-inactivating $\mathrm{Na}^{+}$current. We used patch clamp techniques in neocortical slices to determine whether late $\mathrm{Na}^{+}$ channel openings in somatic patches from Layer 5 pyramidal cells can account for $I_{\text {NaP }}$ as seen in whole cell recordings. In simultaneous cell-attached and whole cell current clamp recordings at the soma, brief $\mathrm{Na}^{+}$channel openings, corresponding to the fast-inactivating $\mathrm{Na}^{+}$current, were always seen during the action potential upstroke. However, there was no persistent $\mathrm{Na}^{+}$ channel activity at potentials at which $I_{\mathrm{NaP}}$ is prominent in whole cell recordings. Kinetic analysis of recordings from multi-channel cell-attached patches at $37^{\circ} \mathrm{C}$ revealed that somatic $\mathrm{Na}^{+}$channels do not demonstrate "modal gating" behavior, and that the probability of single late openings and mini-bursts is extremely low $\left(\mathrm{p}<10^{-5}\right.$ per channel). Local application of TTX $\left(10^{-7}\right)$ only blocked whole-cell recorded $I_{\text {Nap }}$ when puffed near the axon, but was not effective when applied in the region of the apical dendrite or soma. Our data suggest that the cinannels responsible for $\mathrm{I}_{\mathrm{NaP}}$, as seen in whole cell recordings from the soma, are located in the axon.

Supported by a grant from the German-Israeli Foundation for Scientific Research and Development.

Keywords: Na channel; Persistent sodium current; Neocortical neuron

A neural model for motion processing in the visual cortex: analysis of induced motion phenomenon Furman M. and Gur M. Dept. of Biomedical Engineering, Technion, Haifa

The visual images on the retina are constantly moving, due to object, eye and body movements. The ability to estimate objects real motion is crucial for normal functioning of the visual system. In a previous work we presented a physiologically based neuralnetwork model for motion processing in areas V1, middletemporal (MT) and middle-superior-temporal (MST) of the visual cortex. Here, we present an expanded model that include both 
velocity and direction of movement analysis, according to motion selectivity properties of visual cortex neurons. The model was used to simulate the perceptual phenomenon of induced motionthe illusory movement of a static object seen against a moving background. The input to the model depicted a stationary object against a moving textured background, and the responses were interpreted in terms of perceived direction and velocity of movement. The model illustrated how the motion analysis mechanisms used by the visual cortex can account for the perceptual phenomenon of induced motion.

Keywords: Visual cortex, Induced motion, Neural network, Model

Pain behavior and nerve electrophysiology in the CCI model of neuropathic pain.

Gabay E. and Tal M.

Dept. of Anatomy and Cell Biology, Hebrew University-Hadassah, Jerusalem

Rats with an experimental painful peripheral neuropathy created by placing loosely constrictive ligatures around the sciatic nerve (the CCI model) display heat and mechanical allodynia in the affected limb. However, electrophysiological properties of the afferent axons that survive the injury, and hence that are responsible for behavioral responsiveness postoperatively, have not been investigated. Histological studies indicate that the major pathology in $\mathrm{CCI}$ is a loss of large diameter myelinated fibers distal to the ligatures. The question arises therefore, which axons are responsible for the behavioral changes following the injury? Electrophysiological recordings of axons central to the lesion that respond to electrical stimulation distal to the lesion revealed a decrease from $5.2 \pm 0.44$ to $0.54 \pm 0.09$ axons/microfilament 5-9 days post operative (dpo). The value recovered to $1.5 \pm 0.28$ in the $\mathrm{CCI}$ group by $12-15$ dpo. The ratio between $\mathrm{A}$ and $\mathrm{C}$ axons in the control group was $4: 1$ and emained the same in all experimental animals tested, 5-15 dpo. Mechanical stimulation of a distinct area in the paw served by the injured nerve, using von Frey monofilaments, was performed to study how axons in each microfilament were responsive. In the control group $91 \% \pm 0.6$ of the microfilaments had at least one axon with a receptive field. This decreased to $17 \% \pm 2.9$ in the CCI group 5-9 dpo, but recovered to $44 \% \pm 4.2$ by $12-15$-dpo. The threshold for response to mechanical stimulation was not changed by $\mathrm{CCI}$.

Conclusion: In the CCI model there is an equal decrease in the number of $\mathrm{A}$ and $\mathrm{C}$ axons, preserving a constant ratio between the two populations unlike the histologic findings.

Keywords: Nerve injury, Neuropathic pain.

The rhythmogenic capacity of the caudal-lumbar and sacrococcygeal segments of the neonatal rat spinal cord Gabbay H. and Lev-Tov A.

Dept. of Anatomy \& Cell Biology, The Hebrew University School of Medicine, Jerusalem

The rhythmogenic capacity of the tail innervating segments of the spinal cord (L4-Ca3) was studied in isolated spinal cord and tailspinal cord preparations of neonatal rats. Bath application of noradrenaline (NA) or NMDA and NA produced rhythmic sacrococcygeal activity before, and following mid-lumbar transection of the cord. The rhythm could be accelerated by increasing the NMDA concentration, and blocked by bath application of $\alpha 1$ and $\alpha 2$ adrenoceptor antagonists.

The rhythm induced by NMDA/NA in the isolated sacrococcygeal or coccygeal segments of the cord elicited rhythmic tail movements. These movements were produced by an alternating activation of the left and right muscles, and by coactivation of flexors extensors and abductors on a given side of the tail. NMDA/NA also produced a regular rhythmic activity in reduced sacral and coccygeal preparations, and a slower non-alternating rhythm in L4-L6 segments. This L4-L6 rhythm was not coupled to the sacrococcygeal rhythm. Thus, the tail innervating lumbar segments of the cord contributed very little to rhythmogenesis of tail movements.

The NMDA/NA induced rhythm persisted in each half of the midsagittally split sacrococcygeal cord. Therefore, it is suggested that crossed and reciprocal inhibitory pathways are not required for rhythmogenesis of tail movements.

In summary, the rhythmogenic circuitry associated with tail movements is distributed mainly along the sacrococcygeal segments of the spinal cord, and bath application of NA/NMDA was found to be an effective mean to obtain functional activation of this network.

Keywords: Pattern generation, Spinal cord, Noradrenaline.
Rasmussen's encephalitis: a human epilepsy associated with multiple autoantibodies on both sides of the blood-brain

barrier. ${ }^{2}$ Ganor ${ }^{2}$ Amrom D. ${ }^{1}$, Freilinger M. ${ }^{1}$, Goldberg H. ${ }^{1}$, Ben Zeev B. , Verhulpen D. ${ }^{1}$, and Levite M ${ }^{1,2}$

${ }^{1}$ Sackler School of Medicine, Tel Aviv University, Tel Aviv; ${ }^{2}$ Dept

of Neurobiology, Weizmann Institute of Science, Rehovot

Epilepsy has always been considered as a CNS problem. Rasmussen's encephalitis (RE), a severe form of child epilepsy, is the first well-defined CNS disorder in which an autoantibodymediated pathology has been demonstrated: RE patients harbor autoantibodies against the glutamate receptor subtype 3 of AMPA receptors (GluR3). Such anti-GluR3 antibodies, from human, rabbit or mice origin, bind neurons, affect GluR3 function, and cause neuronal death by either an excitotoxic or complementmediated manner. Based on these observations, anti-GluR3 autoantibodies are currently considered as an RE characteristic feature, and used as specific markers for this disease which is often difficult to diagnose.

In a study performed with sera and CSF of six patients with established or highly suspected RE, we found elevated levels of autoantibodies against two defined GluR3 peptides. Most interestingly, the patients CSF and sera harbor a kaleidoscope of other disease-associated autoantibodies: anti-double-stranded DNA Abs, which unlike non-specific anti-ssDNA Abs, recognize the DNA phosphate-deoxyribose backbone, and are found in elevated levels almost exclusively in systemic lupus erythematosus. Furthermore, some RE patients harbor antibodies directed against glutamate decarboxylase as in autoimmune diabetes and stiff-man syndrome, against cardiolipin and $\beta 2$ glycoprotein, as in anti-phospholipid syndrome, and against some nuclear antigens as in various autoimmune diseases. Taken together, our findings show that RE patients harbor not only antiGluR3 antibodies but multiple disease-associated autoantibodies, suggest to treat them as autoimmune patients bearing autoantibodies on both sides of the BBB (i.e in serum and CSF), and shed new light on the connection between autoimmunity and epilepsy.

Keywords: Epilepsy, Autoimmunity, Glutamate receptors;

Rasmussen's encephalitis

\section{Math1- a Molecular Controller of Neural Development} Gazit R. and Ben-Arie N

Dept. of Cell and Animal Biology, Institute of Life Sciences, The Hebrew University of Jerusalem, Jerusalem 91904, Israel.

Transcription factors control the genesis of neurons and glia cells that make the brain. Math1 (mouse atonal homolog 1) is a bHLH transcription activator essential for neurogenesis of the cerebellar granule cells (CGC). In order to characterize functions of Math1 at the cellular level, we study CGC progenitors from knockout (KO) mice in vitro, avoiding complex in vivo secondary effects. Primary cultures of Rhombic Lip (progenitor's birthplace) indicated high viability of CGC cells and that the promoter of Math1 remained active in culture, as indicated by a reporter gene. However, while Math1 expression was normally downregulated with time in control cells, KO cells maintained an abnormal high activity for 6 days. KO cells did not proceed with normal differentiation, in contrast to control that extend neurite in culture. Hence, Math1 has an important intrinsic role in the maturation of CGC and in its negative autoregulation. Finer examination of other cellular and differentiation properties, and the ability of putative downstream genes to rescue the phenotype are in progress. Our studies will enable better understanding of in mammalian neurogenesis and the role of transcription factors in this process.

Keywords: Cerebellar granule cells, Differentitaion, Neurite outhgrowth, Math 1

\section{Structural determinants of external barium block in} homomeric and heteromeric KCNQ1 channels: implications for permeation and gating

Gibor G. ${ }^{1}$, Schottelndreier H. ${ }^{1}$, Peretz A. ' and Attali B. ${ }^{\prime}$

Dept of Physiology \& Pharmacology, Sackler School of Medicine, Tel Aviv University, Tel Aviv 69978

We used the permeant ion $\mathrm{Ba}^{2+}$ to probe the permeation pathway of KCNQ1 channels engaged in homomeric or heteromeric complexes and to evaluate the impact of the $\beta$ subunits on the channel pore properties. Homomeric KCNQ1 and heteromeric $\mathrm{KCNQ} 1 / \mathrm{KCNE} 1$ and $\mathrm{KCNQ} 1 / \mathrm{KCNE} 3$ channels were recorded in Xenopus oocytes, using the two-electrode voltage-clamp 
technique. Extracellular $\mathrm{Ba}^{2+}$ ions produce a time- and voltagedependent block of KCNQ1 currents. For homomeric KCNQ1 channels, $2 \mathrm{mM} \mathrm{Ba}^{2+}$ produce a $35 \%$ block at $+30 \mathrm{mV}$, compared to $68 \%$ block at $-30 \mathrm{mV}$. While the sensitivity to $\mathrm{Ba}^{2+}$ block is very similar for $\mathrm{KCNQ} 1$ and $\mathrm{KCNQ} 1 / \mathrm{KCNE} 1$ channels, the pattern of $\mathrm{Ba}^{2+}$ inhibition is different for the constitutively open heteromeric KCNQ1/KCNE3 channels. At $-140 \mathrm{mV}, 0.5 \mathrm{mM}$ $\mathrm{Ba}^{2+}$ produce only $6 \%$ block of KCNQ $1 / \mathrm{KCNE} 3$ current , while $10 \mathrm{mM} \mathrm{Ba}{ }^{2+}$ elicit a block of $83 \%$. Electrical distance calculation suggest that $\mathrm{Ba}^{2+}$ binds deep in the pore and that at very negative voltages $\mathrm{Ba}^{2+}$ dissociates in the inward direction. In the presence of $\mathrm{Ba}^{2+}$, activation of KCNQ1 and KCNQ1/KCNE3 channels is very slow, suggesting a progressive unblock of $\mathrm{Ba}^{2+}$ ions at positive potentials. Consequently, $\mathrm{Ba}^{2+}$ block switches constitutively open KCNQ1/KCNE3 channels into time- and voltage-dependent channels. $\mathrm{Ba}^{2+}$ ions also affect channel gating by producing a marked rightward shift in the voltage dependence of activation of KCNQ1 and KCNQ1/KCNE1 channels and by accelerating channel deactivation.. The structural determinants of $\mathrm{Ba}^{2+}$ block will be presented and discussed in light of the crystal structure of the homologous bacterial $\mathrm{KcsA} \mathrm{K}^{+}$channel.

Keywords: Potassium channels, Permeation, KCNQ, Barium

Combined administration of dimethyl sulfoxide (DMSO) and haloperidol prevents catalepsy in rats and neuronal toxicity in neuronal cultures.

Gil-Ad I., Shtaif B., Weizman A.

Felsenshtein Medical Research Center. Campus Rabin, Petah

Tiqva,49100 and Tel-Aviv University.

Haloperidol is used in the treatment of schizophrenia and psychotic disorders. Haloperidol therapy is frequently accompanied by extrapyramidal symptoms (EPS). Recent reports suggest the involvement of oxidative insult in the etiology of EPS. DMSO is an organic solvent and a potent free radical scavenger. Haloperidol $1-3 \mathrm{mg} / \mathrm{kg}$ ) was administered I.P to adult male rats. The drug was dissolved in three different vehicles: lactic acid $(1 \%)$, sesame oil and DMSO (0.5-10\%). In the first two vehicles haloperidol induced a marked catalepsy in rats, registered half hourly during three hours. In contrast, in animals treated with haloperidol-DMSO, ho catalepsy was found and no apparent undesired effectss were registered. Addition of vitamin $\mathrm{E}$ did not modify significantly the cataleptic pattern observed with each drug-vehicle solution.

Plasma prolactin levels measured two hours after haloperidol administration were significantly higher compared to basal levels in all the three treatment regimens, suggesting that prevention of cataleptic behavior is not associated with unblockade of dopaminergic receptors

In vitro :Haloperidol (in lactic acid), induced a dose dependent decrease in neuronal cell viability both in primary mouse embryo brain origin, and in human neuroblastoma (SK-N-SH). The drug in DMSO $(0.01-0.1 \%)$ induced a significantly lower neurotoxic effect. Vehicles alone did not modify neuronal cell viability. We conclude that combined administration of haloperidol and DMSO prevents catalepsy in rats without modifying the inhibitory effect of haloperidol on central DA receptors.

We hypothesize that central antioxidative activity of DMSO could account for the protective effect of DMSO against haloperidolinduced catalepsy in rats and neurotoxicity in culture.

Keywords: Haloperidol, Catalepsy, DMSO, Neurotoxicity.

Lysyl oxidase in CNS injury; accelerated functional recovery by inhibitor treatment after spinal cord injury in rodents Gilad G.M. ${ }^{1}$, Kagan H.M. ${ }^{2}$ and Gilad V.H.

Research and Development, Laboratory of Neuroscience, Assaf Harofeh Medical Center, Zrifin 70300, Israel and ${ }^{2}$ Dept. of

Biochemistry, Boston University School of Medicine, Boston, MA, USA

Sites of CNS injuries present a dynamic scene where hematogenous cells, microglia, astrocytes and fibroblasts, interact in a time-dependent manner to perform wound-healing activities, some of which are supportive while others disruptive of axonal regeneration. Modulations of the extracellular matrix (ECM) and scar formation at CNS injury sites are considered prohibitive for successful axon regeneration, thus restricting functional recovery. The present study is based on the premise that lysyl oxidase (LO), an extracellular enzyme that catalyzes cross-linkages of ECM proteins, is involved in modulating the ECM and in scar formation at CNS injury sites, and therefore, its inhibition may be conducive for regeneration and recovery of function. We provide evidence, which indicates that after brain injuries in adult rats, active extracellular LO molecules appear in a spatiotemporal manner in the site of injury and, as indicated by mRNA Northern blot analysis, cells in the region of injury synthesize this enzyme. LO immunoreactivity was not associated with glial fibrillary acidic protein (GFAP)-positive astrocytes. After unilateral spinal cord transection in adult mice, daily treatment (for $20 \mathrm{~d}$ ) with the LO inhibitor, ß-aminopropionitrile $(100 \mathrm{mg} / \mathrm{kg})$, resulted in accelerated and more complete functional recovery. The mode of functional recovery, however, indicated that axonal regeneration of long descending tracts did not occur. The findings indicate that active LO molecules are synthesized and secreted by cells attracted to CNS injury sites and imply that LO may participate in ECM modulation thus hindering recovery after CNS injuries. Keywords: $\beta$-aminopropionitrile, Enzyme inhibitor, Extracellular matrix, Regeneration

\section{Riluzole ameliorates motor function in myelin oligodendrocyte} glycoprotein (MOG)-induced experimental autoimmune encephalomyelitis (EAE)

Gilgun-sherki Y., Offen D., Panet H., Mosberg-Galili R. and Melamed E.

Depts. of Neurology, Felsenstein Medical Research Center, Beilinson Campus and Sackler School of Medicine, Tel Aviv University, and Rabin Medical Center, Petah Tiqva 49100

Glutamate neurotoxicity was shown to play a role in the pathogenesis of multiple sclerosis (MS). Here we study whether treatment with riluzole, an inhibitor of glutamate release, might demonstrate beneficial effect in experimental autoimmune encephalomyelitis (EAE) model induced by myelin oligodendrocyte glycoprotein (MOG). Mice were treated with riluzole $(10 \mathrm{mg} / \mathrm{kg} \times 2 / \mathrm{d}$, i.p. $)$, before, during or after the induction of EAE by the pMOG 35-55. We found that while the control mice developed significant clinical manifestations with complete hind limb paralysis, the riluzole-treated were completely resistant or showed only mild clinical signs. The beneficial effect of riluzole treatment was effective when administrated (i.p.) during or even after the EAE-induction. Histological examination of CNS tissue sections showed multifocal areas of perivascular lymphohistiocytic inflammation with loss of myelin and axons in the control mice while only focal inflammation and minimal axonal damage was demonstrated in the riluzole treated mice. No difference could be detected in the immune potency as indicated by $T$ cell proliferative responses to MOG. In conclusion, we have demonstrated that riluzole attenuate the severity of MOG-induced EAE and reduced axonal damage. Our results suggest that riluzole, and probably other glutamate antagonists may be beneficial in the treatment of MS.

Keywords: Multiple sclerosis, Experimental autoimmune encephalomyelitis (EAE), Myelin oligodendrocyte glycoprotein (MOG), Riluzole

\section{Synaptophysin: purification and characterization of its channel activity}

Gincel D. and Shoshan-Barmatz V.

Dept. of Life Sciences and Zlotowski Center for Neuroscience, Ben-Gurion University of the Negev, Beer-Sheva

Synaptophysin was solubilized from rat brain synaptosomes with relatively low concentration of Triton X-100 (0.2\%) and was purified (95\%) using a single step chromatography on hydroxyapatite/celite column. Purified synaptophysin was reconstituted into planar lipid bilayer and its channel activity was characterized. In asymmetric $\mathrm{KCl}$ solutions (cis $300 \mathrm{mM} /$ trans $100 \mathrm{mM}$ ), synaptophysin forms a fast fluctuating channel with conductance of $414 \pm 13 \mathrm{pS}$ in $+60 \mathrm{mV}$. The open probability of synaptophysin channels was decreased upon depolarization, and channels were found to be cation selective. Synaptophysin channels showed high selectivity for $\mathrm{K}^{+}$over $\mathrm{Cl}^{-}\left(\mathrm{P}_{\mathrm{K}^{+}} / \mathrm{P}_{\mathrm{Cl}^{-}}>8\right)$ and are highly selective for $\mathrm{K}^{+}$over $\mathrm{Li}^{+}, \mathrm{Na}^{+}, \mathrm{Rb}^{+}, \mathrm{Cs}^{+}$, or choline ${ }^{+}$. This is the second demonstration of synaptophysin channel activity, but the first biophysical characterization of its channel properties. The availability of large amount of purified synaptophysin and its characteristic channel properties might help to establish the function of synaptophysin in exocytosis and/or endocytosis.

Keywords: Synaptophysin, Synaptic vesicles, Ion channels 
The relationship between spontaneous activity and functional architecture in primary visual cortex (V1)

Goldberg J.A. and Sompolinsky $\mathrm{H}$.

Racah Institute of Physics and the Center for Neural Computation, Hebrew University, Jerusalem 91904

A recent optical imaging study of the cat V1 demonstrated that spatial maps of cortical activity occurring during spontaneous discharge are highly correlated with the maps evoked by oriented stimuli (Tsodyks et al., Science, 286:1943-6). One interpretation is that in the absence of external stimuli V1 spontaneously switches between a finite set of quasi-stationary cortical states. Alternatively, the intrinsic cortical dynamics yield irregular spatiotemporal patterns whose spatial profiles resemble the responses evoked by oriented stimuli due to the underlying functional architecture of V1.

To resolve this issue, we studied a network model of a patch of V1, consisting of excitatory and inhibitory neurons. Each neuron is labeled by its preferred orientation (PO) and its location on the 2D cortical sheet. The layout of POs on the cortical patch resembles the experimentally observed pinwheel structure. We considered several profiles of intra-cortical interactions. We find that the experimentally observed patterns of spontaneous maps suggest that in contrast to recent suggestions, intra-cortical excitatory connections depend not only on distance between neurons but also on the difference between their POs. This architecture combined with strong neuronal adaptation yields irregularly evolving patterns of activity that resemble the observed characteristics of the spontaneous maps. Thus, spontaneous activity yields important information on the architecture and dynamics of the cortical circuits.

Keywords: Dynamics, Attractors, Cortical feedback, Orientation

The spatial organization of electrically coupled networks of interneurons in neocortex.

Golomb D. and Amitai Y.

Dept. of Physiology, Faculty of Health Sciences, Ben-Gurion

University, Beer Sheva.

Inhibitory interneurons of the neocortex are electrically coupled to cells of the same type through gap junctions. We studied the spatial organization of two types of interneurons in the rat somatosensory cortex: fast-spiking (FS) parvalbuminimmunoreactive $(\mathrm{PV}+)$ cells, and low threshold-spiking (LTS) somatostatin-immunoreactive (SS+) cells. Paired recordings in layer 4 demonstrated that both the probability of coupling and the coupling coefficient drop steeply with intersomatic distance. The dendritic arbors of FS and LTS cells were reconstructed from electrophysiologically characterized, biocytin-filled cells; the two cell types had only minor differences in the number and span of their dendrites. However there were marked differences in the density and spatial distributions of the two interneuron types: in general, there was a higher density of PV+ cells than SS+ cells $\left(92 \pm 9\right.$ and $67 \pm 12$ cells $/ \mathrm{mm}^{2}$ respectively). PV+ cells were especially dense in layer 4 , while SS+ cell density peaked in the subgranular layers. From these data we calculate that each interneuron is electrically coupled, directly or indirectly, to between 20 and 50 other interneurons. The large number of electrical synapses implies that each interneuron type participates in a continuous syncytium over wide areas. To evaluate the functional significance of these findings, we examined several simple architectures of coupled networks analytically. We present a mathematical method to estimate the average electrical coupling each cell receives from its neighbors, and the average leak conductance of cells, and we estimate that these have the same order of magnitude: around $10 \mathrm{nS}$. These quantitative results have important implications for the effects of electrical coupling on the dynamic behavior of interneuron networks.

Keywords: FS cells, LTS cells, Gap junctions, Dendritic fields, Coupling coefficient, Coupling conductance, Network architecture

The role of interleukin-1 in hippocampal-dependent memory processes and neural plasticity Goshen II. Kemsler A. ${ }^{3}$ Iverfeldt K. ${ }^{I}$ Dept. of Psychology, The Hebrew University of Jerusalem, Jerusalem, Israel; ${ }^{2}$ Dept. of Psychology, Haifa University, Haifa, Israel; ${ }^{3}$ Dept. of Neurobiology, Weizmann Institute of Science, Rehovot, Israel; ${ }^{4}$ Dept. of Neurochemistry and Neurotoxicology, Stockholm University, Stockholm, Sweden

Interleukin-1 (IL-1) is a pleiotropic cytokine, produced by immune cells in the periphery as well as glia and neurons within the brain. To examine the role of IL-1 signaling pathways in the physiological mechanisms underlying learning memory and neural plasticity we used two models of disturbed IL-1 signaling: Mice with targeted deletion of the IL-1 receptor type I (IL-1r KO) and mice with transgenic over-expression of brain IL-1 recepto antagonist (IL-1raTG). Because TNF $\alpha$ and IL-1 were previously shown to have many common functions, we also examined mice with a targeted deletion of the TNF type I receptor (TNFrKO). Compared to their respective wild type controls, IL-1rKO and IL1raTG mice, but not TNFrKO mice, displayed significantly disrupted memory, when tested with a spatial memory paradigm of the Morris water maze (MWM), known to require normal hippocampal functioning. When tested with a non-spatial memory paradigm (visible platform), which does not lean on hippocampal mechanisms, IL-1rKO and IL-1raTG mice showed no memory impairment. Short-term plasticity in dentate gyrus (DG) and CA1 synapses of IL-1rKO mice was disrupted, as evidenced by enhanced paired-pulse inhibition and suppressed paired-pulse facilitation, both in vivo and in vitro. Long-term plasticity was completely absent in these mice, which displayed no LTP following high frequency stimulation, either in the DG of anesthetized animals, or in CA1 of hippocampal slices. These results suggest that signaling via the IL-1 type I receptor within the hippocampus plays a critical role in learning and memory processes as well as in short- and long-term plasticity.

Partly supported by the Smith Laboratory for Collaborative

Research in Psychobiology.

Keywords: Interleukin-1 (IL-1), Memory, LTP, Hippocampus

Rapid efflux into blood contributes to the elimination of excess glutamate in brain.

Gottlieb M. and Teichberg V.L.

Dept. of Neurobiology, The Weizmann Institute of Science,

Rehovot 76100, Israel.

The brain extensive vascularization has traditionally been seen as the main supply route to the brain of obligatory metabolites and as a barrier barring the entry into the brain of potentially harmful molecules. We wish here to provide evidence for an additional prominent role of the brain vascularization which is to protect the brain by allowing the rapid elimination to blood of the excitotoxic neurotransmitter glutamate. We have followed the efflux into blood of $\left[{ }^{3} \mathrm{H}\right]$ glutamate after its injection into the rat brain ventricular system. The levels of $\left.{ }^{3} \mathrm{H}\right]$ glutamate increase in blood immediately after the injection and reach a plateau. The initial rate of efflux corresponds to $0.6 \pm 0.1 \%$ per minute of the $\left[{ }^{3} \mathrm{H}\right]$ glutamate input and decreases to $0.2 \pm 0.05 \%$ per minute after $20 \mathrm{~min}$ of the $\left[{ }^{3} \mathrm{H}\right]$ glutamate ICV administration reflecting the diffusion and uptake of $\left[{ }^{3} \mathrm{H}\right]$ glutamate into brain tissue and the decrease of its free concentration around the brain capillaries. These rates are decreased by the concomitant intraventricular injection of non labeled glutamate indicating that the latter and $\left[{ }^{3} \mathrm{H}\right]$ glutamate compete for the same glutamate transporters on the antiluminal side of the capillary endothelium. Though glutamate is transported from the brain to blood, this process takes place in spite of a very unfavorable gradient since the glutamate concentration in blood is around $180 \mu \mathrm{M}$ and that in the CSF around $1 \mu \mathrm{M}$. We propose that the elimination of synaptic or excitotoxic glutamate takes place not only into neuronal or glial compartments but also into blood via the extremely dense brain capillary network.

Keywords: Brain-blood efflux, Glutamate, Transport, Neuroprotection

\section{NAP: mechanism of femtomolar neuroprotection through} immunomodulation

Gozes I. ${ }^{1}$, Romano J. ${ }^{1}$, Zaltzman R. ${ }^{1}$, Beni-Adani L. ${ }^{2}$, Beni S.M. ${ }^{3}$, Steingart R.A. , Ashur-Fabian O. ${ }^{1}$ Levy Nissenbaum O. ${ }^{4}$ Brenneman D.E. ${ }^{5}$ and Shohami E

${ }^{I}$ Dept. of Clinical Biochemistry, Sackler Faculty of Medicine, Tel Aviv University, Tel Aviv 69978, Israel; ${ }^{2}$ Sourasky Medical Center, Tel Aviv University: ${ }^{3}$ Pharmacology, The Hebrew University School of Pharmacy, Jerusalem $91120 ;{ }^{4}$ Life Science, Tel Aviv University, Israel; ${ }^{5}$ Section on Developmental and Molecular Pharmacology, LDN, NICHD, NIH, Bethesda, MD 20892, USA.

The eight-amino-acid peptide (NAP), derived from ActivityDependent Neuroprotective Protein - ADNP (J. Neurochem. 72: 1283-1293 [1999]), provides femtomolar neuroprotection. Recent studies suggest that part of NAP activity may be indirect through 
immunomodulation. Thus, NAP attenuates tumor necrosis (TNF $\alpha$ ) secretion immediately after head trauma and protects against TNF $\alpha$-associated cell death (J. Pharmacol. Pharm. Exp. Therap. 296:57-63 [2001]). Utilizing gene atlas arrays, we discovered that closed head injury $(\mathrm{CHI})$ is associated with the upregulation of Mac-1, an adhesion molecule of the integrin $\beta 2$ family (CD11b/CD18), and a major factor in the complement system. $15 \mathrm{~min}$. after injury, mice were divided into two groups, control and NAP-treated and a single subcutaneous injection of NAP or vehicle was administered. A third group served as shamtreated. Each mouse was assessed for its clinical function, using neurological severity score. 30-45 days after CHI, total cerebral cortex RNA was prepared from the site of injury andfrom parallel regions in peptide-treated and sham brains. Reversed transcription yielded radioactive cDNA preparations, that were further hybridized to Atlas array membranes containing 1200 cDNAs spots (Clontech). Results indicated that Mac-1 mRNA specifically increased after $\mathrm{CHI}$ and decreased after NAP treatment. In a different set of experiments, NAP was chronically injected for the first 3 weeks of life to apolipoprotein E-deficient mice exposed to head trauma at 4 months. RT-PCR analysis indicatedthat Mac-1 expression increased after head trauma and was inhibited by NAP treatment. Thus, Mac-1 is suggested as a marker for the long-term outcome of head injury and as a potential target for NAP protective actions.

Support: ISF, BSF, Neufeld, ISOA, Gildor Chair

Keywords: Trauma, Neuroprotection, Mac-1, ADNP.

\section{Rectification of biochemical and behavioral abnormalities} manifested in an animal model for depression

Green T., Deri I., Zangen A., Yadid G.

Faculty of L ife Sciences, Bar Ilan University, Israel

The brain mechanisms underlying depressive symptoms, such as anhedonia, are evasive and need to be elucidated. FSL rats are a genetic animal model of depression that exhibit increased anhedonia in response to chronic mild stress, and there are substantial alterations in their serotonergic and catacholaminergic functions. We examined whether antidepressant treatment could alter the reward circuits, the functions of which are based on abnormal neurophysiological activity in FSL rats. We found that extracellular dopamine levels in FSL rats did not increase in response to serotonin stimulation, as opposed to control rats. However, chronic antidepressant treatment normalized the serotonin-dopamine interaction. Moreover, FSL rats exhibited a lower increase in extracellular $\beta$-endorphin levels in response to serotonin stimulation, as opposed to control rats. However, the serotonin- $\beta$-endorphin interaction of FSL rats was restored following chronic antidepressant treatment. In addition, behavioral deficiencies in FSL rats, manifested as increased immobility during a swim test, were significantly improved following desipramine treatment. Finally, we examined the reinforcement effect of cocaine on FSL rats, using the self-administration paradigm. Our results show that the great majority of FSL rats did not proceed to acquire cocaine via self-administration. Following chronic treatment with desipramine, FSL rats were allowed to selfadminister cocaine. We found that $70 \%$ of FSL rats responded to cocaine. We suggest that treatment with desipramine may have restored the serotonin-dopamine- $\beta$-endorphin cross-talk in FSL rats, and thus normalized the response of FSL rats to cocaine. Thus, this model may prove useful for examining reward systems in states of anhedonia.

Keywords: Depressive behavior, Serotonin, Dopamine, $\beta$ endorphin, Cocaine self-administration

\section{Incidence of different cell death mechanisms in the newborn} rat retina

Guimarães C.A. and Linden R.

Programa de Neurobiologia - Instituto de Biofisica da UFRJ, $C C S /$ bloco $G$, Ilha do Fundão, 21949-900, Rio de Janeiro, RJ, Brasil

Introduction: There are different types of programmed cell death occuring either in physiological and pathological processes. Two of them, apoptosis and autophagy, share some halmarks as cell shrinkage and plasmatic membrane intergrity maintainance. Anisomycin (ANI), a protein synthesis inhibitor, induces cell death in recent post-mitotic cells of the newborn retina. Degenerating cells are condensed, heavily stained with neutral red and are found among healthy looking cells, sugesting plasmatic membrane integrity.
Objectives: Our aim in this study is to identify different types of cell death induced by anisomycin in the retinal tissue and their mechanisms of execution.

Methods: Newborn retinal explants were kept for $24 \mathrm{~h}$ in vitro in control conditions or in the presence of cell death inhibitors. Explants were fixed, tissue criosections were made and processed for histology or immunocytochemistry and analysed under light microscopy.

Results: Bongkrekic acid, a permeability transition pore inhibitor, completely inhibited cell death induced by anisomycin. AcLEHD-CHO, a caspase-9 inhibitor peptide, Z-DEVD-FMK, a caspase- 3 inhibitor peptide, and Ac-VEID-CHO, a caspase- 6 inhibitor, inhibited aproximately $50 \%$ of cell death induced by ANI. Furthermore, immunocytochemistry for activated caspase-3 and TUNEL stained aproximately $50 \%$ of dead cells in tissue sections. Co-incubation of retinal explants with caspase- 3 and caspase- 6 inhibitors did not have a synergic effect, but the coincubation with caspase- 9 and caspase- 6 inhibitors completely blocked cell death induced by anisomycin. 3-methyl-adenine (3MA), an autophagy inhibitor, also reduced cell death induced by anisomycin to the same levels reached with apoptosis inhibitors described above. Co-incubation of retinal explants with autophagy and apoptosis inhibitors did not have a synergic protective effect and TUNEL staining decreases in explants treated with $3 \mathrm{MA}$

Conclusions: ANI induces at least 2 types of cell death, both dependent on mitochondrial committment and caspase activation. The first one is caspase-9 and caspase-3-dependent and involves an autophagic process. The second type of cell death involves caspase-6 activation and possibly corresponds to the TUNEL negative cells. According to our results, apoptosis and autophagy seem to be different stages of the same cell death process, sugesting an overlap of these two features.

\section{Principles of neocortical interneuron recruitment}

Gupta A. ${ }^{1}$, Wang $\mathrm{Y}^{2}$ and Markram $\mathrm{H}$.

${ }^{\top}$ Dept. of Neurobiology, Weizmann Institute of Science, 76100 Rehovot, Israel; ' Section of Neurobiology, Yale University School of Medicine, 333 Cedar Street, New Haven, CT 06520-8001, USA GABAergic interneurons constitute only a minor fraction (20$30 \%$ ) of neocortical cells, but are essential for normal brain function. Recent studies have expanded our knowledge of how this small and highly diverse population of cells, inhibit neighboring neurons, but the properties of their recruitment are still largely unknown. We therefore obtained simultaneous wholecell patch clamp recordings $(n>120)$ from several anatomical and electrophysiological distinct types of interneurons receiving convergent inputs from up to three presynaptic pyramidal cells (PCs) in layers 2-4 of rat neocortical slices. Glutamatergic synapses formed by PCs onto interneurons were diverse in their dynamic properties, being either facilitating or depressing. Morphologically distinct interneuron types were found to receive both types of synapses. However, distinct electrophysiological subclasses of a given morphological interneuron type invariably received either only facilitating or depressing synapses (synapse mapping principle). Indeed, inputs from several PCs converging onto a single interneuron target all form synapses of the same type $(n>25)$, and single pyramidal neurons innervating different electrophysiological interneuron subclasses (divergence) differed in their temporal dynamics (differential synaptic transmission). Facilitating synapses varied widely in their underlying kinetic properties and synaptic strenghts: some target interneurons could be discharged by a single presynaptic PC, whereas others could not be discharged by even three convergent PC-inputs. Interneurons, therefore, differ in their thresholds for recruitment, alluding to functionally unique positions within the neocortical microcircuitry during network activity. Finally, in many cases PCs were found to be reciprocally connected to their target interneurons indicating that many interneurons may directly affect the population of PCs responsible for their initial recruitment. Our findings show that innervation of interneurons by PCs follows distinct organizational principles suggesting that each type of interneuron is uniquely recruited during activity of the neocortical microcircuitry.

Keywords: Neocortex, Interneurons, Pyramidal cells, Recruitment 
A dissociation between brain activity and perception: fMRI responses to chromatic flicker that is not perceived Gur M. ${ }^{1}$ and Grienfeld A

${ }^{1}$ Dept. of Biomedical Engineering, Technion, Israel

When two isoluminant colors alternate at frequencies $>10 \mathrm{~Hz}$, we perceive only one fused color with a minimal sensation of brightness flicker. In an earlier work (Gur and Snodderly, 1997) we have shown that color opponent cells in V1 of the alert monkey follow isoluminant flicker at 15 and $30 \mathrm{~Hz}$. Thus there was cortical activity that represented visual stimulation, yet was not perceived. Here we present an fMRI study performed on human observers at the Functional Brain Imaging Laboratory, TelAviv Sourasky Medical Center. Subjects viewed red/green flickering lights that were fused to a steady yellow percept at 15 $\mathrm{Hz}$, while flicker was very clear at the lower frequencies of 2.5 and $7.5 \mathrm{~Hz}$. There was activity in area 17 in response to color flicker at the 2 low frequencies as well as at $15 \mathrm{~Hz}$ where no color or flicker was perceived. Thus in humans as in monkeys primary visual cortex is active but, seemingly, this activity fails to affect conscious perception. The implication of our findings to theories about the relation of brain activity and consciousness will be discussed.

Keywords: Visual cortex, Perception, Color flicker, fMRI,

Consciousness

The in vivo targeting of a magnetite-bound lentiviral vector by an extra-corporeal magnetic field

Haim $\mathrm{H}^{1}{ }^{1}$, Panet $\mathrm{A}^{2}$ and Steiner I.

${ }^{7}$ Laboratory of Neurovirology, Dept. of Neurology, Hadassah

University Hospital, Jerusalem; ${ }^{2}$ Dept. of Virology, The Hebrew

University-Hadassah School of Medicine, Jerusalem,

Current in vivo gene transfer technology is generally limited to the local application of the gene-carrying vector to the target site. The targeted delivery of a systemically administered vector to selected tissues continues to pose a major challenge. Obstacles include the immune response, hemodynamic parameters affecting vector biodistribution and inefficient impartion of host-cell range selectivity. In order to surmount the above and achieve sufficient cellular transduction at the target site following systemic administration, we attempted a novel method of gene transfer. Magnetite nanoparticles ( $50 \mathrm{~nm}$ in diameter), coated by a hydrophilic polymer matrix with endstanding cationic DEAE groups, electrostatically bind to the anioniz envelope of most viruses. Using a lentiviral vector carrying the $\beta$-galactosidase reporter gene, we exposed cell cultures to the virus-magnetite complex and demonstrated localization of infection to the cells overlying a magnet positioned under the culture dish. Transduction efficiency was significantly increased. Examining the ability of the complex to mediate organ specific transduction, we are currently conducting the first in vivo experiments. Balb/c mice are intravenously injected mice with $10^{7}$ infectious units, simultaneously exposing the lateral cranium to a magnetic field for the first 10 minutes post-injection. One week later the animals are sacrificed and brains removed, fixated and stained by X-gal. Transduction efficiency relative to control mice is quantified by measurement of ONPG tissue levels. The results of the in vivo experiment will be presented.

Keywords: Gene therapy, Nervous system, Lentivirus, Targeting, Magnetite nanoparticles.

Direct interactions of two voltage-gated $\mathrm{K}^{+}$channels, Kv1.1 and Kv2.1 with proteins of the exocytotic apparatus; syntaxin $1 A$ and SNAP-25

Harari S. Chikvashvili D. and Lotan I.

Dept. of Physiology and Pharmacology, Sackler School of Medicine, Tel-Aviv University, Tel Aviv

Recently, we suggested that direct interaction between voltagegated $\mathrm{K}^{+}(\mathrm{Kv})$ channels and proteins of the exocytotic machinery may contribute to neurotransmitter release (Fili et al., 2001). We could demonstrate that a physical interaction between syntaxin 1A and the $\mathrm{Kv1} 1 \mathrm{1} / \mathrm{Kv} \beta$ channel occurred in fresh synaptosomes, involved also other protein component of the exocytotic machinery, synaptotagmin and SNAP-25, and was altered following the triggering of transmitter release. Further, we defined the interaction between the $\mathrm{Kv} 1.1 / \mathrm{Kv} \beta 1.1$ channel and syntaxin $1 \mathrm{~A}$ in Xenopus oocytes and in vitro. Here we extended the study in oocytes and in vitro and characterized the interaction of $\mathrm{Kv1} 1 / \mathrm{Kv} \beta 1.1$ with SNAP-25. We show that SNAP-25 coprecipitates with the channel from whole oocytes and plasma membranes. SNAP-25 interacts in vitro with both $\mathrm{Kv} \beta 1.1$ and the $\mathrm{N}$-terminus of Kv1.1. The sites of interaction within the $\mathrm{N}$ terminus are further characterized and are compared to those of syntaxin 1A. Also we extend the study to another $\mathrm{Kv}$ channel belonging to another subfamily, Kv2.1. This channel coprecipitates with syntaxin $1 \mathrm{~A}$ in synaptosomes (personal communication by Yaniv Bledi and Michal Linial). We show that Kv2.1 co-precipitates with syntaxin and SNAP-25 from oocytes and can bind in vitro syntaxin and SNAP-25.

Keywords: Kv channels, Syntaxin, SNAP-25

The wasp Ampulex Compressa injects venom directly into prey central nervous system

Haspel G., Rosenberg L. and Libersat F.

Zlotowski Center for Neuroscience and Department of Life

Sciences, Ben-Gurion University, Beer-Sheva

In contrast to other venomous animals that paralyze their prey, the venomous parasitoid wasp Ampulex Compressa subdues its prey by the induction of a bout of grooming followed by a five-week long lethargic state. During the lethargic state the cockroach is docile enough for the wasp to pull it into a burrow where the wasp larva eats it alive. The cockroach is always stung by the wasp twice, first in the thorax and then in the head

To localize the site of stinging we injected wasps with a mixture of $\mathrm{C} 14$ radiolabeled amino acids. After the amino acids were incorporated into the venom, we allowed the wasps to freely sting several cockroaches.

First, the amount of radioactivity in the ganglia of stung cockroaches was assessed using liquid scintillation of the different ganglia and tissue. Significantly higher levels of radioactivity were detected in the head ganglia and the first thoracic ganglion. In contrast, radioactivity levels in the third thoracic ganglion and non-neuronal tissue were much lower and comparable to control values.

Second, microscopic emulsion autoradiography was carried out to determine the precise location of injection. Radioactivity was detected in the central area of the brain ganglion, the subesophageal ganglion and the first thoracic ganglion. No radioactivity could be detected in the second thoracic ganglion or in ganglia stung by non-radiolabeled wasps.

To our knowledge, this is the first direct evidence of a venomous animal stinging into the central nervous system of its prey.

Keywords: Venom, Wasp, Cockroach, Central-body

Acuity demand as a driving force in the organization of highlevel object areas

Hasson U. ${ }_{1}$, Levy I. ${ }^{1,2}$, Avidan G. ${ }^{1,2}$, Behrmann M. ${ }^{3}$ Hendler T. ${ }^{4,5}$ Malach R.

${ }^{1}$ Weizmann Institute of Science, Rehovot Israel; ${ }^{2}$ Hebrew

University, Jerusalem, Israel; ${ }^{3}$ Carnegie Mellon, Pittsburgh, PA, USA; ${ }^{4}$ Sourasky Medical Center, Tel Aviv, Israel; ${ }^{5}$ Tel Aviv University, Tel Aviv, Israel

We have recently proposed center-periphery organizing principle in which object representations are laid-out in the cortex according to resolution needs. Thus, objects whose recognition depends on detailed scrutiny (e.g. faces) will be associated with foveal-biased representation, while objects whose recognition depend on lowresolution, large-scale integration (e.g. buildings) will be associated with periphery-biased representation. A direct prediction stemming from this hypothesis is that each object category must be associated with a unique profile of eccentricity preferences, based on the acuity which is needed for their recognition. In this study we tested this hypothesis by examining the relationship of center/periphery organization to the representation of letters and words, whose recognition depends crucially on foveal vision. As controls, we tested additional object categories whose recognition is not obviously dependent on foveal vision (computers, cars). Our result revealed a strong association between letters, words and central visual field representations, while the activation to computers and cars was distributed towards peripheral-biased regions. These results indicate that the resolution-based organizing principle can predict the activation pattern of different object categories within high-level object areas.

Funded by Israel Academy 644/99-1 grant.

Keywords: AMRI, Object recognition, Ventral stream. 
T cell-based therapeutic vaccination for traumatic spinal cord injury

Hauben E., Mizrahi T., Agranov E. and Schwartz M.

Dept. of Neurobiology, The Weizmann Institute of Science,

\section{Rehovot}

The outcome of incomplete spinal cord injury (ISCI) is often more severe than expected, because in addition to the degeneration induced directly by the insult, the inury triggers a self-destructive process leading to secondary degeneration of initially spared neurons. Neuronal degeneration after injury to the rat optic nerve or spinal cord can be reduced by passive or active $T$ cell-based immunization with myelin-associated self-antigens such as myelin basic protein (MBP) (Hauben et al, $J$ Neuroscience, 20:6421-30 [2000]). This "protective autoimmunity" plays a physiological role in the spontaneous recovery from CNS trauma (Yoles et al, $J$ Neuroscience, 21:3740-8 [2001]). Here we show that (a) mice devoid of mature $\mathrm{T}$ cells (nude mice) recover significantly worse from ISCI than the wild type, and (b) female rats and mice, which are inherently capable of sustaining a stronger post-traumatic $T$ cell response than males, recover significantly better than males from ISCI. We also describe a post-traumatic vaccination protocol for promoting functional recovery from ISCI. To boost protective autoimmunity through vaccination, with minimal risk of inducing experimental autoimmune encephalomyelitis (EAE), we used altered MBP peptides in which the replacement of one amino acid by another renders the peptide non-encephalitogenic. Rats vaccinated, immediately after ISCI, with the altered MBP peptide did not develop EAE symptoms and their functional outcome was significantly improved (Hauben et al, J Clinical Investigation, 108(4):591-9 [2001]). Several myelin-derived peptides, including Nogo, were effective in inducing a benign T-cell-mediated neuroprotective response. Accurate interpretation of the role of autoimmunity in CNS recovery may lead to the development of a therapeutic vaccine for treatment of ISCI.

Keywords: Neuroprotection, Autoimmunity, Paralysis, Myelin.

Generalization and the evolution of internal states of neural microcircuits

Häusler S. ${ }^{1}$, Maass W. ${ }^{1}$ and Markram $\mathrm{H}^{2}$

Institute for Theoretical Computer Science, Technische

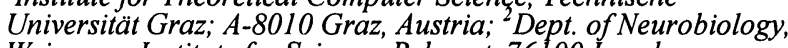

Weizmann Institute for Science, Rehovot, 76100 Israel

Liquid state machines store salient information about previous input patterns in form of high dimensional complex dynamical states, whereas their readout transforms equivalent states to a stable output. We have examined the ensembles of trajectories of internal states caused by similar inputs, to which a readout neuron after training is supposed to assign theoretical same output. More precisely, we have analyzed to what extent these trajectories share some common properties. In order to measure this quantitatively we have compared these ensembles of trajectories of internal states of a simulated neural microcircuit with randomly drawn arbitrary internal states of the same circuit, that were not caused by related circuit inputs. Furthermore we have analyzed through computer simulations the generalization capabilities of such simulated neural microcircuits. We have tested to what extent a readout neuron can give correct inputs to novel inputs, both to inputs that were generated by the same distribution as the training inputs, and to inputs which differ essentially from those inputs on which the system had been trained.

Keywords: Microcircuits, Generalization, Dynamical systems

Classification power of readout neurons in neural microcircuits

Häusler S. ., Maass W. ${ }^{1}$ and Markram $\mathrm{H}^{2}$

Institute for Theoretical Computer Science, Technische

Universität Graz; A-8010 Graz, Austria; ${ }^{2}$ Dept. of Neurobiology, Weizmann Institute for Science, Rehovot, 76 I00 Israel

Recently Maass, Markram \& Natschläger proposed a new theoretical model, called "liquid state machine", which represents a convenient framework for neural computations in real time for rapidly time varying input functions. The liquid may for example be a computer model of a recurrent neural microcircuit, whose complex dynamical state serves as a source for readout neurons to extract salient information about past and present stimuli. Their capability to transform different transient internal states of the liquid into given target outputs strongly depends on how well trajectories of different input streams can be separated in the high dimensional state space. We show that if time-varying inputs are injected into a sufficiently large recurrent neural microcircuit, a single readout neuron is in principle able to classify the inputs. This effect is well-known in the machine learning community (since support vector machines rely on it), but has hardly been explored in the context of computational neuroscience. We have quantitatively analyzed the classification power of a single readout neuron in dependence of the size of the recurrent neural microcircuit into which the input has been injected. In order to test the potential classification power of a single readout neuron we studied how well different input patterns could be linearly separated in the state space of the liquid. For this purpose we used Fisher's linear discriminant analysis to find the linear transformation (projection) to a low dimensional subspace that separates the different sets of liquid states in an optimal way and determined the hyperplane orthogonal to this transformation with the least classification error. Further we studied the dependence on various parameters like the structure and the size of the liquid, the neuron types, the synaptic properties as well as the special form of the input patterns. The study also demonstrates the capability of recurrent neural microcircuits to store and retrieve memory in real time without stable states.

Keywords: Neural coding, Pattern classification

\section{Molecular diversity of pyramidal cells}

Herzberg C., Toledo-Rodriguez M., Mae S.L., and Markram H. Dept. of Neurobiology, Weizmann Institute of Science, Rehovot Pyramidal cells are excitatory (glutamatergic) cells and the most abundant $(70-80 \%)$ neurons in the neocortex. This cell type exhibits a stereotypical morphology in layer 2-5 and most pyramidal cells display similar discharge patterns. Pyramidal cells in layer 6 may display diverse morphologies and a small fraction of pyramidal cells through the layers exhibit different electrophysiological behaviors. Very little is known about the molecular diversity of pyramidal cells. We recently developed single cell multiplex RT-PCR protocols that allow simultaneous detection of mRNAs for around 40 proteins from neurons morphologically and electrophysiologically characterized. The mRNA profile includes 3 calcium binding proteins, 9 neuropeptides, 4 enzymes and over 30 ion channel alpha and beta subunits. Whole-cell patch clamp recordings from pyramidal cells in all neocortical layers were performed in somatosensory cortex neocortical slices of juvenile rats (P13-P16). After whole-cell the electrophysiological properties of the cells were studied in detail, the neuron was loaded with biocytin for subsequent $3 \mathrm{D}$ anatomical computer reconstructions and at the end of the experiment cytoplasm was aspirated for subsequent single cell RT-PCR.

Keywords: Pyramidal neurons, Single cell RT-PCR, Ion channel, Electrophysiology.

\section{Chronic treatment from weaning with amitriptyline prevents} hyperanxiety and depressive-like behavior in prenatallystressed rats.

Herzog-Raalbag P., Poltyrev T., Bejar C. and Weinstock M. Dept. of Pharmacology, School of Pharmacy, Hebrew University of Jerusalem, Israel

Adult offspring of rats stressed during the last week of pregnancy display heightened anxiety in intimidating environments and a faster acquisition of immobile posture than controls [C] in the forced swim test (FST). The aim of this study was to see whether an antidepressant, amitriptyline could reduce the symptoms of anxiety and depression in prenatally-stressed. [PS] rats, when given daily from weaning to adulthood. To avoid handling, water or amitriptyline $(4.5 \mathrm{mg} / \mathrm{kg} / \mathrm{day})$ was administered to PS and C rats in the drinking water, from the age of 3 to 8 weeks. The rats' behavior was assessed at 10 weeks in a mildly stressful environment, the open field (OF) and in the plus maze, (PM) a validated test for anxiety. PS rats given water showed significantly less exploration than $\mathrm{C}$ in the OF and spent less time in the open arms of the PM, indicating hyperanxiety; PS, $2.6 \pm 1.4 \mathrm{sec}$; C, $11.5 \pm 3.1$ sec. PS rats given amitriptyline showed significantly more exploration in the $\mathrm{OF}$ and spent much more time $(40.0 \pm 9.7$ sec, $\mathrm{P}<0.01$ ) in the open arms of $\mathrm{PM}$, resembling behavior induced by diazepam. By contrast, amitriptyline had no effect in $\mathrm{C}$ rats. In FST, amitriptyline also decreased immobility in PS rats from 210 \pm 17 to $135 \pm 21 \mathrm{sec}(\mathrm{P}<0.01)$ but increased immobility in $C$ rats from $131 \pm 10$ to $189 \pm 4 \mathrm{sec}$. In conclusion, the data show that early treatment with amitriptyline abolishes the symptoms of hyperanxiety and depression induced by prenatal stress.

Keywords: Prenatal stress; Anxiety; Depression; Amitriptyline. 
Auditory Cortex Specialization Processing Temporal Aspects of Natural Speech: Onset Time of Voicing vs. Analogous NonSpeech Stimuli

Horev N. ${ }^{2}$, Most T. ${ }^{2}$ and Pratt $\mathrm{H}^{1}$

${ }^{7}$ Evoked Potentials Laboratory, Technion-Israel Institute of

Technology, Haifa; ${ }^{2}$ Dept. of Communication Disorders, School

Of Health Professions, Tel Aviv University.

Speech is thought to be perceived and processed in a unique way by the human auditory system. In this study we show evidence for different brain processing of speech and analogous non-speech stimuli.

Cortical auditory evoked potentials (AEPs) were obtained from Hebrew-speaking listeners using 21 scalp electrodes in an active identification task.

Speech stimuli were selected from a naturally produced $/ \mathrm{ba} /-/ \mathrm{pa} /$ continuum that varied in voice-onset-time (VOT), which refers to the time between the stop burst and the onset of voicing. Nonspeech stimuli were selected from a synthesized two-formants continuum that varied in the onset time of the first formant in relation to the second formant. (Formant-onset-time - FOT). The formants frequencies and the intensity of the FOT stimuli were adjusted to match the spectra of the speech stimuli and to elicit similar behavioral responses.

Analysis of the AEPs suggested that the differentiation in processing speech and non-speech signals starts as early as $100 \mathrm{~ms}$ after stimulus onset: VOT values had a significant effect on primary auditory cortex activity, whereas FOT values affected later activity. In addition, the non-speech stimuli of $+15 \mathrm{~ms}$ FOT evoked larger late responses, which might reflect the innate (not speech-specific) sensitivity for onset-asynchronies of this magnitude. This effect was reduced in response to the parallel VOT stimulus, presumably as a result of the acquired specialization in processing language-specific acoustic cues.

Source estimation mapped these effects to the auditory cortices showing different lateralization patterns depending on stimulus type (speech/non-speech) and stage of processing.

Keywords: Evoked potentials, Hemispheric specialization, Auditory cortex

Changes in morphology and electrophysiology of neurons and glial cells in mouse sensory ganglia following partial obstruction of colon

Huang TY. and Hanani M.

Hebrew University-Hadassah Medical School, Mount Scopus,

\section{Jeruselam 91240}

Obstruction of visceral organs induces hypertrophy of neurons innervating these organs, but there is little information about the changes in electrical properties of the neurons and in the characteristics of glial cells in the corresponding sensory ganglia. In this study we investigated the morphological and electrophysiological changes in neurons and satellite glial cells (SCs) of mouse dorsal root ganglia (DRG) after obstruction of the colon. We first studied the sensory projections to mouse colon using Dil retrograde tracing and found that both DRG L1 and S2 innervate the colon. After 6 days of obstruction we observed the following cellular changes: 1. The thresholds for firing an action potential, membrane potentials and input resistances of the neurons were significantly lower than controls. The proportion of neurons with spontaneous action potentials increased about 4-fold in $\mathrm{S} 2$ and $\mathrm{L} 1$. The proportion of neurons with subthreshold potential oscillations increased by $116 \%$ in S2 and $102 \%$ in L1. 2 . About $14 \%$ of SCs in S2 and $11 \%$ in L1 became dye coupled to SCs surrounding neighboring neurons and thus formed dye coupling between glial envelopes. 3. The cross-sectional area of $\mathrm{DRG}$ neurons increased by $35 \%$. 4. Inflammatory responses were observed within the colon wall. These results suggest that obstruction-induced inflammation caused hyperexcitability and hypertrophy of the neurons and dye coupling between glial envelopes. These changes are similar to those observed in DRGs after axotomy of sciatic nerves. We propose that changes in DRGs contribute to visceral pain, as is the case for somatic pain.

Keywords: Dorsal root ganglia, Glial cells, Intestinal obstruction, Electrophysiology

\section{Stress and pain responses of rats lacking cholecystokinin $A$} (CCKA) receptors.

Hurwitz I. Avnon Y.L. ${ }^{2}$, Malkesman O. ${ }^{1,2}$, David A. ${ }^{1}$ and Weller A.

${ }^{I}$ Interdisciplinary Program in the Brain Sciences and ${ }^{2}$ Dept. of Psychology, Bar Ilan University, Ramat-Gan, Israel.

CCK mediates several behavioral effects, including stress induced ultrasonic vocal responses (USV) of infant rats (reduced via the
$\mathrm{CCK}_{\mathrm{A}}$ receptor) and opioid-analgesia (modulated via the $\mathrm{CCK}_{\mathrm{B}}$ receptor). In this study we studied CCK involvement in stressresponsiveness by using Otsuka Long Evans Tokushima Fatty (OLETF) rats that do not express $\mathrm{CCK}_{\mathrm{A}}$ receptors and comparing their responses to Long Evans Tokushima (LETO) control rats. 11-12-day-old OLETF and LETO pups from 10 and 14 litters, respectively, were tested in the isolation test and number of USV were counted. The protocol was $2 \mathrm{~min}$ in an empty container, then $8 \mathrm{~min}$ with either water or milk available. In addition, young adult OLETF and LETO rats (63-70 days old) were challenged with a moderate thermal pain stimulus by immersing the tail in hot water $\left(49 C^{\circ}\right.$ or $\left.53 C^{\circ}\right)$ and measuring the tail flick response. Rate of USV (USV/min) was significantly greater ( 5 fold) during the first 2 min, and during the subsequent $8 \mathrm{~min}$ (12 fold) in the container with water, in OLETF compared to LETO pups. In the milk test condition, USV rate of OLETF pups was reduced, and was not different from controls. Adult OLETF rats reacted to thermal pain significantly faster than controls at both test temperatures. The results support previous findings, suggesting that CCK mediates infant vocal responsiveness through the $\mathrm{CCK}_{\mathrm{A}}$ receptor. The hyperalgesia found in OLETF rats may be due to an alteration in the number or sensitivity of $\mathrm{CCK}_{\mathrm{B}}$ receptors

Supported by the Israel Science Foundation.

Keywords: USV, Pain, CCK.

\section{Immunomodulation of experimental autoimmune}

encephalomyelitis by recombinant human alpha-fetoprotein Irony-Tur-Sinai M. and Brenner T.

Laboratory of Neuroimmunology, Dept. of Neurology, HadassahHebrew University Medical Center, Jerusalem

Alpha-fetoprotein (AFP) is an immunomodulating glycoprotein that is synthesized during embryonic development by the fetal liver and the yolk sac. AFP concentrations in maternal human serum increase gradually in the second and third trimesters of pregnancy and decline rapidly at the time of delivery. The immunomodulatory effects of AFP on both humoral and cellular immune responses have been described in detail. Clinical remissions during the second half of pregnancy have been reported in several autoimmune diseases, such as myasthenia gravis (MG), multiple sclerosis, rheumatoid arthritis, systemic lupus erythromatosis and thyroiditis, and may be attributed to the immunosuppressive effect of AFP. Furthermore, we previously showed that human AFP, isolated from human maternal or fetal serum, suppresses experimental autoimmune encephalomyelitis (EAE) and experimental autoimmune MG. Recently, following the synthesis of immunoactive recombinant human AFP (rhAFP), cloned and expressed in large quantities in E.coli, we tested this preparation in vivo and in vitro. Our findings show that treatment with rhAFP of mice during induction of EAE prevents the development of the disease. The effect was dose-dependent; large concentrations of AFP completely protected the treated animals from EAE, and lower doses conferred partial protection. AFP serum levels of treated mice were comparable to that of women at the third trimester of pregnancy. In addition, rhAFP suppressed Tcell response to the encephalitogenic autoantigen myelin oligodendrocyte glycoprotein and to the mitogens phytohemagglutinin and concanavalin $\mathrm{A}$. We hope that as a result of our studies, AFP, a naturally occurring immunomodulatory glycoprotein, will be considered a candidate in the management of human autoimmune diseases.

Keywords: Alpha-fetoprotein, Experimental autoimmune encephalomyelitis, T-cell reactivity, Immunomodulation

Plasticity of Neuronal Firing Mode by Regulation of a T-Type

Ca $^{2+}$ Channel
Jiang Y. and Yaari Y.

${ }^{2}$ Dept. of Physiology, Hebrew University-Hadassah School of Medicine, Jerusalem, Israel, ${ }^{2}$ Dept. of Neuropathology, ${ }^{3}$ Dept. of Epileptology, University of Bonn Medical Center, Bonn, Germany. The potential for activity-dependent plasticity is a fundamental property of the brain. While activity-dependent, long-term changes in synaptic function have been studied intensely, persistent alterations in intrinsic firing properties and their cellular mechanisms have received only minor attention.

Previous analysis of the firing behavior of CA1 hippocampal neurons following a $2 \mathrm{~h}$ period of status epilepticus (SE), induced by injection of the convulsant pilocarpine, has shown that most neurons were converted from regular to burst firing neurons 
(Sanabria et al, J. Physiol. 532:205-216 [2001]). The fraction of bursterjs reached a maximum at day 5 (64\% compared to $<3 \%$ in control animals) following $\mathrm{SE}$ and decreased thereafter over a time course of several weeks. The novel bursting was not blocked by the $\mathrm{Ca}^{2+}$ channel antagonists nifedipine, $\omega$-conotoxin GVIA or MVIIC or $\omega$-agatoxin TK, but proved highly sensitive to low concentrations of $\mathrm{Ni}^{2+}$ known to block $\mathrm{T}-/ \mathrm{R}-\mathrm{type} \mathrm{Ca}^{2+}$ channels. To test whether up-regulation of either R- or T-type $\mathrm{Ca}^{2+}$ channels underlies novel intrinsic bursting, we have recorded these current subtypes in isolated CA1 neurons and in hippocampal slices using patch-clamp techniques. The T-type $\mathrm{Ca}^{2+}$ current proved 2.4 -fold increased, while the R-type current was not significantly altered. These changes were accompanied by a significant increase in $\alpha_{1 \mathrm{H}}$, but not $\alpha_{1 \mathrm{E}}, \alpha_{1 \mathrm{G}}$, or $\alpha_{1 \mathrm{I}}$ subunit mRNA levels determined by realtime RT-PCR on day 3 after SE.

These data suggest that an episode of intense neural activity induces a persistent change in the firing mode of CAl neurons that may be caused by the transcriptional up-regulation of the $\alpha_{1 \mathrm{H}} \mathrm{Ca}^{2+}$ channel subunit.

Supported by DFG (SFB 400 \& Be 2078/1-1), BMBF/MOS, ISF, the Humboldt Foundation and BONFOR

Keywords: Burst-firing, Calcium channels, Pyramidal cells, Hippocampus, Epilepsy, Rat

\section{'Compulsive' lever pressing after post-training signal attenuation is abolished by fluoxetine and increased by lesion to the orbital cortex: A novel rat model of obsessive compulsive disorder?}

Joel D., Avisar A. and Doljansky J.

Dept. of Psychology, Tel-Aviv University, Tel Aviv 69978

We (Joel \& Avisar, Behavioural Brain Research 123:77-87 [2001]) have recently developed a new rat model of obsessive compulsive disorder (OCD) based on the hypothesis that obsessions and compulsions result from a deficient response feedback mechanism or a deficient signaling that the conditions have changed following the organism's response. As a result, the successful completion of an action does not lead to its cessation, as would normally occur. In our procedure, attenuation of an external feedback for operant behavior results in excessive emission of this behavior without an attempt to collect a reward. The latter suggests that the lever-press behavior is not only excessive but also "inappropriate" or "unreasonable", thus fulfilling DSM-IV criteria for compulsive behavior. In line with this hypothesis, 'compulsive' lever-pressing (1) is abolished by the serotonin re-uptake inhibitor, fluoxetine, but not by the anxiolytic drug, diazepam, in accord with the differential efficacy of these drugs in treating OCD patients; (2) is affected by manipulations of the dopaminergic system (Joel et al, Behavioral Neuroscience [in press]), in line with findings implicating abnormalities of this system in OCD; and (3) is enhanced following lesion to the orbital cortex but not to the medial prefrontal cortex, in line with findings implicating the orbitofrontal cortex but not the dorsolateral prefrontal cortex in the production of obsessions and compulsions. These results provide an initial foundation for a novel rat model of OCD.

Keywords: Obsessive compulsive disorder (OCD); Animal model; Operant behavior

\section{Corneal nerve damage in diabetic rats - correlation between} sensitivity and morphology

Kadar T. ${ }^{\mathrm{P}}$, Glover JP. ${ }^{1}$, Zuckerman A. ${ }^{2}$, Hermelin R. and Robison WG jrit

'National Eye Institute, National Institutes of Health, Bethesda, MD 20892, USA; ${ }^{2}$ Israel Institute for Biological Research, Ness Ziona

Decreased sensitivity is a typical phenomenon of the diabetic cornea. However, its pathogenesis is still not clear. The present study was aimed to characterize the clinical and morphological alterations of corneal innervation in the galactose-fed rat model of diabetic ocular complications. Corneal sensitivity and the structure of corneal nerves were investigated in galactosemic rats and in aged-matched control animals (24 months old), and compared to corneal function in normal eight month-old control animals. Corneal sensitivity was measured by means of a Cochet-bonnet aesthesiometer, using the blink reflex as an objective parameter. Whole-mount flat corneas, stained by the Karnovsky method for acetylcholinesterase activity were used for light microscopy observations and computerized morphometric analysis of neural density.

Corneal sensitivity declined significantly with age and further deterioration was seen in the galactosemic rats. Age-related morphological changes were found in the corneal nerve structure of the 24 months old rats, expressed mostly as a dense network of fine regenerative fibers originating from the subepithelial plexus. This regenerative process was not seen in the galactosemic corneas, where the density of the nerves was markedly reduced. In addition, most galactosemic corneas exhibited a unique pattern of irregular blister-like axonal swellings, suggested to be an expression of frustrated regeneration. A close relationship was found between the corneal nerve density and the degree of sensitivity. It is concluded that the abnormal corneal nerve changes and loss of corneal epithelial nerve endings might be the basis for the clinical abnormalities, reported in the diabetic cornea. Keywords: Corneal innervation, Diabetes, Acetylcholinesterase (AChE)

In V1 duplex cells, the form of responses to gratings depends on temporal frequency

Kagan I. ${ }^{1}$ Gur M. ${ }^{1,2}$ and Snodderly D.M. ${ }^{2,3}$

Dept. of Biomedical Engineering, Technion, Haifa, Israel; ${ }^{2}$ Schepens Eye Research Institute; and ${ }^{3}$ Dept. of Ophthalmology and Program in Neuroscience, Harvard Medical School, Boston $M A$

Our earlier studies have shown that responses to gratings of "duplex" cells, the dominant type in V1 of alert monkeys, are diverse and can not be predicted from receptive fields' spatial maps. These cells have overlapping increment and decrement activating regions (ARs), but many have a significant fundamental (F1) harmonic in responses to drifting sinusoidal luminance gratings. Conversely, transient stimuli, such as flashing bars and counterphase gratings, evoke mostly on-off, or frequency doubled (second harmonic, F2) responses. This mixture of "linear" and "nonlinear" properties suggests that the temporal dynamics of interactions between increment and decrement ARs and the suppressive surround play an important role in shaping duplex cells responses. Therefore, in this study we used gratings of varying temporal frequency to study the responses of duplex cells in parafoveal V1 of a monkey performing a fixation task. We have found that many cells responded with a significant linear (F1) modulation to high temporal frequency gratings, but show frequency doubled (F2) responses at low temporal frequencies. This temporal frequency-dependent F2 modulation differs from low spatial frequency doubling that we observed in a large portion of duplex cells. These results reveal an elaborate spatiotemporal structure of duplex cells receptive fields.

Keywords: Primary visual cortex, Gratings, Spatial frequency, Temporal frequency

Association between the cell cycle and neural crest delamination through specific regulation of G1/S transition Kalcheim C. and Burstyn-Cohen T. Dept. of Anatomy and Cell Biology. Hebrew University of Jerusalem-Hadassah Medical School. Jerusalem 91120

Delamination of premigratory neural crest cells from the dorsal neural tube depends upon a gradient of BMP4 activity along the neuraxis which is generated by changing levels of noggin. The latter are in turn modulated by an inhibitory activity from the dorsomedial somite which coordinates the timing of cell emigration with the elaboration of a migratory mesodermal substrate. Cell-intrinsic mechanisms also regulate delamination. Here we show that neural crest cells synchronously emigrate from the neural tube in the S-phase of the cell cycle. Specific inhibition of the transition from G1 to $\mathrm{S}$ both in vivo and in vitro blocks delamination, whereas inhibition at $S$ or $G 2$ phases has no immediate effect. Thus, the transition between $\mathrm{G} 1$ to $\mathrm{S}$ is necessary for the epithelial-to-mesenchymal conversion of crest precursors and may be required for the cells to respond to environmental signals that trigger delamination.

Keywords: Neural cest, Control of proliferation, Migration, Morphogenesis. 
Micromolar concentrations of hydrogen peroxide modulate neuronal plasticity In CA1 region of rat hippocampus in a bimodal manner

Kamsler A. and Segal $\mathrm{M}$.

Dept. of Neurobiology, Weizmann Institute of Science, Rehovot 76100

Hydrogen Peroxide $\left(\mathrm{H}_{2} \mathrm{O}_{2}\right)$ is produced in cells as a by product of oxygen metabolism. Excess amounts of $\mathrm{H}_{2} \mathrm{O}_{2}$ are toxic and may underlie neurodegenerative diseases such as Alzheimer's and Parkinson's diseases. We have examined the effect of $\mathrm{H}_{2} \mathrm{O}_{2}$ on neuronal transmission and synaptic plasticity in CA1 region of rat hippocampus slices. We found a bi-modal, concentration dependent effect of $\mathrm{H}_{2} \mathrm{O}_{2}$ on synaptic physiology. High concentration of $\mathrm{H}_{2} \mathrm{O}_{2}(5 \mathrm{mM})$ drastically reduces all neuronal activity as is indicated by a marked suppression of excitatory post synaptic potentials (EPSP's). Lower concentration of $\mathrm{H}_{2} \mathrm{O}_{2}(20$ $\mu \mathrm{M})$ did not affect baseline properties of EPSP's but prevented Long Term Potentiation (LTP) of reactivity to afferent stimulation following a tetanic train of stimuli. This concentration also inhibited the phosphorylation of extracellular regulated kinase (ERK) which is necessary for LTP induction. Conversely, applying a similar protocol of stimulation under even lower concentration of $\mathrm{H}_{2} \mathrm{O}_{2}(1 \mu \mathrm{M})$ resulted in LTP that was increased two fold compared to control. Such potentiation could be achieved in control slices by multiple trains of stimulation. We are currently investigating the molecular mechanisms underlying this dual action of $\mathrm{H}_{2} \mathrm{O}_{2}$. Our data suggest a role for $\mathrm{H}_{2} \mathrm{O}_{2}$ as a second messenger in signal transduction cascades that control neuronal excitability

Keywords: Hydrogen peroxide, Synaptic plasticity, Hippocampus

Induction of experimental antiphospholipid syndrome with CNS involvement in 5 different mouse strains

Katzav A. ${ }^{1}$, Pick CG. ${ }^{2}$, Korczyn A.D. ${ }^{1,3}$, Litvinjuk Y. ${ }^{1,5}$, Shrot $S^{1}{ }^{1}$ Lenz D. ${ }^{1}$, Blank M. ${ }^{4}$, Sirota P. ${ }^{5}$, Shoenfeld $\mathrm{Y}^{4}$ and Chapman J. ${ }^{1,3}$ Depts. of Physiology and Pharmacology, Anatomy ${ }^{2}$, and Neurology, Sackler Faculty of Medicine, Tel Aviv University, Ramat Aviv 69978; , ${ }^{4}$ Dept. of Medicine B and Research Unit of Autoimmune Diseases, Sheba Medical Center, Tel Hashomer 52621; ${ }^{5}$ Abarbanel Mental Health Center, Bat Yam

Objective: The antiphospholipid syndrome (APS) includes systemic and central nervous system (CNS) pathology associated with antibodies to a complex of phospholipids and $\beta_{2}$-glycoprotein I $\left(\beta_{2}\right.$-GPI). We have recently reported the induction of APS associated with behavioral and cognitive deficits in Balb/C female mice that developed 4-5 months following immunization with $\beta_{2-}$ GPI. In the present study, we examined the influence of genetic factors on the ability to induce experimental APS with CNS involvement by testing several mouse strains immunized with $\beta_{2}$ GPI. Methods: Female mice from five strains were immunized once with $\beta_{2}$-GPI in complete Freund's adjuvant (CFA) or with CFA alone (controls). Neurological assessment in a staircase test and in a T-maze alternation test was performed 4-5 month following the immunization. Results: Immunization with $\beta_{2}$-GPI resulted in elevated levels of antibodies against negatively charged phospholipids and $\beta_{2}$-GPI in all five mouse strains. Autoantibody levels were significantly higher in $\mathrm{Balb} / \mathrm{C}, \mathrm{ICR}$, and $\mathrm{C} 57 / \mathrm{Bl}$ mouse strains compared to $\mathrm{AKR}$ and $\mathrm{C} 3 \mathrm{H} / \mathrm{eb}$ mouse strains. Hyperactivity reflected by higher number of stairs climbed in 3 minutes, was seen in the $\mathrm{Balb} / \mathrm{C}$, ICR, and $\mathrm{C} 3 \mathrm{H} / \mathrm{eb}$ mouse strains. Anxiety reflected by more frequent rears, was seen in the $\mathrm{Balb} / \mathrm{C}$, $\mathrm{AKR}$, and $\mathrm{C} 3 \mathrm{H} / \mathrm{eb}$ mouse strains. Cognitive decline in the T-maze alternation test was seen in the $\mathrm{Balb} / \mathrm{C}, \mathrm{C} 3 \mathrm{H} / \mathrm{eb}$, and $\mathrm{C} 57 / \mathrm{Bl}$ mouse strains. Conclusion: In this study we demonstrate the ability to induce APS with CNS involvement in different mouse strains. These results indicate that there is no clear correlation between autoantibody levels and neurological impairment, and suggest a complex genetic immune interaction.

Learning and memory affecting aplysia feeding: nitric oxide and protein synapsis

Katzoff A., Shochat M. and Susswein A.J.

Faculty of Life Sciences, Bar Ilan University, Ramat Gan 52900

We have examined the roles of Nitric Oxide (NO), and protein synthesis, in learning and memory affecting Aplysia feeding. Aplysia were trained with an inedible food. Aplysia orient to the food, bite and attempt to swallow it, before rejecting it, and then stopping to respond. Memory is shown by a reduced response to the food. The role of NO was examined by using L-NAME, a competitive inhibitor of $\mathrm{L}$ - arginine for NO Synthase, and by using the NO scavenger PTIO. Protein synthesis was blocked with anisomycin.

Treatment with L-NAME or PTIO before training with inedible food did not affect orientation and feeding responses during training, and animals learned to stop responding to the food. However, short term $(0.5 \mathrm{hr})$, intermediate-term $(4 \mathrm{hr})$ and longterm $(24 \mathrm{hr})$ memories were all blocked, indicating that NO is required for all forms of memory.

Treatment with anisomycin before training with inedible food did not affect training Short-term memory was also not significantly different from that in control animals. However, long-term memory was blocked

We also examined the effects of blocking NO transmission or protein synthesis following training. Injecting L-NAME immediately after training had no effect on either short- or longterm memory. By contrast, injecting anisomycin immediately following training blocked long-term, but not short-term memory. Thus, NO transmission and protein synthesis have different roles in memory formation. NO is needed for all forms of memory, but only during training, whereas protein synthesis is needed only for long-term memory, during the consolidation period.

Keywords: Nitric Oxide, Protein synthesis, Learning and memory.

Peptides of affiliation: oxytocin advances weaning in the rat, vasopressin does not.

Kavushansky A. and Leshem M.

Dept. of Psychology, Haifa Universi3ty. Haifa

Weaning enables the infant to separate from its mother, consolidate other social interactions, and forage and explore. We believe this "behavioral metamorphosis" is mediated by changes in the neurochemical substrates of attachment and affiliation, such as the oxytocin and vasopressin systems examined here. Eighteen-day-old rat pups ('sucklings') and thirty-five-day-old 'weanlings' were given intracerebroventricular injections of oxytocin, its antagonist (OTA) (both $0.5 \mu \mathrm{g} / 1 \mu \mathrm{l}$ ), vasopressin (lng), its antagonist (VPA) $(0.5 \mu \mathrm{g})$ or vehicle. Pups were tested in a behavioral maze monitoring exploration, and responses to their anesthetized dam or awake sibling. The only age-dependent effects were that oxytocin completely abolished nipple attachment in sucklings, and in weanlings, OTA increased time spent near the dam. We conclude that the vasopressin system does not influence weaning as tested in our maze (although other effects on affiliation were observe4d). Oxytocin contributes to weaning by inhibiting nipple attachment a the initiation of weaning, and, in weanlings, reducing the need for proximity to the dam. In psychological parlance this could be interpreted as respectively reducing the infant's need for 'contact comfort' and a 'secure base', thereby promoting separation from the dam.

Keywords: Affiliative behavior, Oxytocin, Vasopressin,

Weaning, Rat.

Presenilin 1 independently regulates $\beta$-catenin stability and transcriptional activity

Killick R. ${ }^{1}$, Pollard C.L. ${ }^{1}$, Asuni A.A. ${ }^{1}$, Mudher A.K. ${ }^{1}$

Richardson J.C. ${ }^{2}$, Rupniak H.T.R. ${ }^{2}$, Lovestone S. ${ }^{1}$ and Anderton B.H. ${ }^{1}$

${ }^{I}$ Dept. of Neuroscience, Institute of Psychiatry, King's College London, De Crespigny Park, Denmark Hill, London, SE5 8AF United Kingdom.; 'GlaxoSmithKline Research and Development Ltd, Gunnels Wood Road, Stevenage, Hertfordshire, SGI $2 N Y$

United Kingdom

Presenilin 1 (PS1) regulates $\beta$-catenin stability, however, published reports regarding the direction of its effect are contradictory. We have examined the effects of wild-type and mutant forms of PS1 on the membrane, cytoplasmic, nuclear and signaling pools of endogenous and exogenous $\beta$-catenin by immunofluorescence microscopy, subcellular fractionation and in a $\beta$-catenin/Tcf dependant transcription assay. We found that PS1 destabilizes the cytoplasmic and nuclear pools of $\beta$-catenin when they are stabilised by Wnt or Dvl but not when stabilization occurs at lower levels of the Wnt pathway (FRAT1 overexpression and $\mathrm{LiCl}$ inhibition of GSK-3 $\beta$ ). The PS1 mutants we examined were all less able to inhibit the stability of $\beta$-catenin. PS1 also inhibited the transcriptional activity of endogenous $\beta$-catenin and the PS1 mutants were again less inhibitory at the level of Dvl but showed a different pattern of inhibition towards transcription below Dvl. The transcriptional activity of exogenously expressed wild-type $\beta$ catenin and a truncated form, $\triangle \mathrm{N} 89 \beta$-catenin which lacks the 
GSK-3 $\beta$ phosphorylation sites, were also inhibited by wild-type and mutant PS1. We conclude that PS1 negatively regulates the stability and transcriptional activity of $\beta$-catenin at different levels in the Wnt pathway, that the effect on transcriptional activity appears to be independent of GSK-3 $\beta$ mediated degradation of $\beta$ catenin and that mutations in PS1 differentially affect the stability and transcriptional activity of $\beta$-catenin.

Keywords: Alzheimer's, Wnt, $\beta$-catenin.

Protective and destructive autoimmunity in CNS degenerative disorders common players and different regulation

Kipnis J., Yoles E., Shaked I. and Schwartz M.

Dept. of Neurobiology, Weizmann Institute of Science, Rehovot 76100

Experimentally induced neuroprotection mediated by endogenous autoreactive $\mathrm{T}$ cells was recently shown in our laboratory to be a physiological response to central nervous system (CNS) insult. This finding revealed an apparent conflicting effect of these encephalitogenic $T$ cells, causing autoimmune diseases on one hand and protecting neurons from post-traumatic degeneration on the other. In this study we show that myelin-associated autoreactive $\mathrm{T}$ cells are essential for endogenous neuroprotection, but are effective only in combination with regulatory $\mathrm{T}$ cells, which on their own, however, are insufficient for neuroprotection. When this multicellular mechanism is missing or malfunctioning, as in the case of individuals or strains with a genetic propensity to develop autoimmune disease, the insult-evoked response mediated by encephalitogenic $T$ cells may lead to autoimmune disease rather than neuroprotection. Our findings imply that beneficial autoimmunity and the resistance to autoimmune diseases are regulated by a common mechanism. Future animal studies of nerve regeneration and neurotrauma should take into account the genetically determined ability of particular strains to exhibit endogenous neuroprotection (Kipnis, J., et.al. 2001. J Neurosci. 21:4564-4571; Schwartz, M., and J. Kipnis. 2001. Trends Mol Med. 7:252-258). These findings compelled us to modify our understanding of autoimmunity and autoimmune diseases, as well as the role of autoimmunity in non-autoimmune CNS disorders.

Keywords: Neuroprotection; CNS; EAE; Beneficial autoimmunity

Salt preference in patients with $\mathrm{CAH}-21-\mathrm{OH}$ deficiency congenital adrenal hyperplasia).

Kochli A. Rakover Y. , and Leshem M.

Dept. of Psychology, Haifa University. 'Pediatric Endocrine Unit, Ha'Emek Medical Center, Afula

little is known about the determinants of salt intake in humans. It is commonly believed that increased salt intake in humans is due to availability and dietary habits established in infancy. However, studies have failed to confirm this belief. In contrast, research in animals has shown that salt intake is determined by physiological and genetic factors. In humans, the only reports of physiologically-related salt appetite have been sporadic and anecdotal, in cases of Addison's disease. Here we report on a systematic study of salt appetite in $\mathrm{CAH}$ patients with various mutations of the $\mathrm{CAH}-2 \mathrm{l}-\mathrm{OH}$ gene. We compared their salt appetite to that of their relatives, both carriers and non-carriers. Preliminary results of our study suggest that salt preference may be increased in some cases.

Keywords: Congenital adrenal hyperplasia, Genetic, Salt, Salt intake

\section{Developmental aspects of clustering in verbal fluency in}

Hebrew

Koren R., Kofman O. and Berger A.

Dept. of Behavioral Sciences and Zlotowski Center for Neuroscience, Ben-Gurion University of the Negev, Beer Sheva

Verbal fluency is an executive function (EF) task that requires the timed production of words according to specific criteria. Phonological fluency involves naming words beginning with a certain letter and semantic fluency involves naming words belonging to a particular semantic category. The fluency task requires that the participant search for words from the appropriate sub-category, produce the words, and switch to another subcategory. Healthy participants spontaneously cluster words according to phonological cues (first sound, rhyme, etc.) or semantic cues (sub-categories). Clustering has been used as a measure of EF in brain-damaged adults such as Parkinson's disease and Alzheimer's disease patients (Troyer et al. JINS 4:137-143 [1998]). Developmental aspects of fluency were tested in children in grades 3 and 5 using the sounds ר, ג, פי, שי,
$(\mathrm{R}, \mathrm{G}, \mathrm{P}, \mathrm{SH}, \mathrm{D})$ for the phonological fluency and the categories animals, food, clothing, things that are seen on the street for semantic fluency. The number of words produced, number of clusters, cluster size, repetition errors and rule break errors were analyzed for differences between grade and gender. It was hypothesized that there would be greater fluency, more frequent and longer clusters and fewer errors in the fifth grade pupils. Verbal fluency was higher in fifth grade pupils, as was the number of clusters in the semantic, but not phonological fluency task. The findings confirm the hypothesis that the ability to produce clusters, which depends on the frontal lobe increases with development Keywords: Phonological fluency, Semantic fluency, Executive functions, Development.

Blood-Brain Barrier Disruption In Humans Is Associated With Abnormal Cortical Rhythm Generation and Neurological Signs and Symptoms

Korn A. ${ }^{-}$Golan $\mathrm{H}^{2}$, and Friedman A. ${ }^{1}$

Dept.s of Physiology and Neurosurgery, Ben Gurion University and Soroka Medical Center, Zlotowski Center of Neuroscience, Beersheva, 84105, Israel. ${ }^{2}$ Nuclear Medicine Dept., Rabin Medical Center, Golda-Hasharon Campus, Petach-Tikva, Israel.

Numerous pathological diseases of the human central nervous system have been reported to involve perturbation of blood-brainbarrier (BBB) integrity. Both the mechanisms underlying BBB disruption and the physiological and functional consequences of such pathology are poorly understood. We report a series of 13 patients, aged 15-54, who were found to have a focal BBB disruption in single photon emission computerized- tomography (SPECT) following injection of $99 \mathrm{mTc}-$ Diethylenetriaminepentaacetic Acid (Tc-DTPA). Of these patients, 5 were 6-12 months post-surgery for removal of a benign meningioma, 5 were post-mild head injury, 2 suffered from idiopathic seizures and one was 36 months post-radiosurgery for an arterio-venous malformation. All patients displayed symptoms that were consistent with partial or complex seizures, but none were symptomatic during the examination. Except for BBB disruption, no other gross anatomical lesions were found in brain $\mathrm{CT}$ or magnetic resonance imaging (MRI). To explore the effects of BBB disruption on cortical activity, high-density 128 channel electroencephalography was collected. Spectral analysis revealed abnormally high-powered slow wave $(3-6 \mathrm{~Hz})$ activity in all patients. In 9 patients, low-resolution-electrotomography (LORETA) localized the slow activity to originate in the same region as the BBB lesion. Regions of abnormal activity were significantly larger in patients displaying prolonged clinical history, despite similar size of their BBB lesions. Our data suggest that focal BBB lesions, as the solely detected anatomical brain pathology, may adversely affect cortical activity. Future studies are needed to clarify which clinical disorders may be associated with pathologies of brain microvasculature, and what is their natural history.

Keywords: Blood Brain Barrier, SPECT, Spectral analysis, LORETA

Molecular basis of cerebellar granule cell development Krizhanovsky V. and Ben-Arie $\mathrm{N}$

Dept. of Cell and Animal Biology, Institute of Life Sciences, The Hebrew University of Jerusalem, Jerusalem 91904, Israel.

The cerebellum is essential for coordination of movement and posture, and its dysfunction disrupts balance and impairs control of speech, limb and eye movements. Hence, normal development, survival and well being of the cerebellar neurons are essential for maintaining proper cerebellar function. One of the key genes for cerebellar development is Mathl, which encodes a basic helixloop-helix transcription factor. Mathl is expressed in cerebellar granule cells precursors and their deviates, and is essential for their generation, as identified in Mathl knock out mice. To characterize molecular mechanisms controlling the generation and survival of cerebellar granule cells we aimed at identifying Math I target genes. We utilized DNA arrays to compare multiple gene expression in cerebella of Mathl knockout and control mice. From 1200 genes tested, 28 were differentially expressed. A confirmatory semi-quantitative RT-PCR was performed on 17 of them, of which 14 displayed different expression between Math1 null and wild type cerebella. The deferentially expressed genes participate in proliferation, apoptosis and patterning, while others are transcription factors and neuronal specific genes. To identify possible Math1 direct targets we performed a database search for putative Mathl binding sites in genomic sequences flanking their 
human orthologs. In four of the identified genes, a sequence very similar to Mathl autoregulatory binding site was found. Hence, these genes may be putative direct Mathl targets. This is first identification of Mathl direct targets, thus our results shed light on cerebellar granule neuron development at the molecular level. Keywords: Math1, Cerebellar granule cells, Transcription factor, Target genes.

\section{Modulation of neuronal plasticity relevant genes, CAM-L1} and laminin, in rat brain, by antidepressants and stress Laifenfeld D. ${ }^{1}$, Karry R. ${ }^{1}$, Grauer E. ${ }^{2}$, Klein E. . , Ben-Shachar D. ${ }^{1}$ Taboratory of Psychobiology and Dept. of Psychiatry, Rambam Medical Center and B.Rappaport Faculty of Medicine, Technion ${ }^{2}$ Dept. of Pharmacology, Israel institute of biological research, Ness Ziona.

Stressful life events have been implicated as a predisposing factor in depression, and altered noradrenergic transmission has been observed in both stress and depression. Recent research focusing on an involvement of long term intracellular processes in stress as well as in response to antidepressant treatment led us to study long term noradrenergic effects on neuronal plasticity and relevant gene expression. We have previously reported a differentiating role for $\mathrm{NE}$ on the SH-SY5Y noradrenergic cell-line, including an ability to promote neurite outgrowth in these cells, accompanied by a NE induced increase in the expression of the cell adhesion molecule CAM-L1 and of laminin, interacting proteins that play a role in neuronal differentiation and neurite outgrowth. Presently, we studied alterations in the expression levels of CAM-L1 and laminin in healthy rats treated with either the noradrenergic antidepressant desipramine or the SSRI fluoxetine. We found consistent alterations in the expression levels of CAM-L1 and laminin, which differed according to brain region (hippocampus, frontal cortex, striatum). Moreover, we found that rats exposed for 6 weeks to variable unpredictable stress, previously suggested as an animal model for post-traumatic-stress-disorder (PTSD), also exhibited altered expression of both CAM-L1 and laminin, in a brain region specific manner and in a pattern different from that observed consequent to antidepressant treatment. Given the ability of NE to regulate the above-mentioned genes, we suggest a possible mechanism whereby nroadrenergic regulation of genes involved in synaptic connectivity may be associated with the pathophysiology and the treatment of depression.

Keywords: Depression, Stress, Norepinephrine, Neural cell adhesion L1, Laminin

\section{Mechanisms of cholinergic sensitivity to neurotoxic stimuli} Landman N. ${ }^{1}$, Greenberger V. ${ }^{1}$, Groner Y. ${ }^{2}$ and Segal M. Depts. of Neurobiology and Molecular Genetics', Weizmann institute of Science, Rehovot 76100

The cholinergic system of the basal forebrain has been shown to play an intricate role in normal learning and memory function. Its significance is exemplified by the fact that selective neurodegeneration of cholinergic neurons in Alzheimer's disease (AD) strongly correlates with the degree of cognitive impairment. However, most of the AD research thus far failed to determine the underlying mechanisms of selective cholinergic cell death.

We hypothesized that oxidative stress is a potential underlying mechanism of cholinergic degeneration. Immunohistochemical examination of the basal forebrain cholinergic system in brain sections of Tg-SOD1 mice, a model of oxidative stress, showed a $30-40 \%$ lower number of ChAT-positive neurons in Tg-SOD1 mice as compared to age-matched controls. We then used prenatal (D19) cultures from normal rat basal forebrains to assess the sensitivity of basal forebrain cholinergic neurons (BFCN) to neurotoxic stimuli. At 10 or 14 days post plating, cultures were exposed to various concentrations of $\mathrm{H}_{2} \mathrm{O}_{2}$ or NMDA (10-100 $\mu \mathrm{M})$. Low doses of $\mathrm{H}_{2} \mathrm{O}_{2}(10-30 \mu \mathrm{M})$ or NMDA $(10-50 \mu \mathrm{M})$, which did not affect survival of non-cholinergic neurons, resulted in degeneration of $50 \%$ of ChAT-positive neurons. These results suggest that BFCN are hypersensitive to neurotoxic stimuli.

Various neurotoxic stimuli have been shown to exert their effect through changes in calcium homeostasis. To examine the potential role of calcium-related mechanisms in BFCN degeneration, basal forebrain cultures were exposed to the calcium ionophore A23187. This treatment resulted in decreased survival of cholinergic vs. non-cholinergic neurons, suggesting that there is a difference between the two cell populations in the ability to handle sustained calcium load. This possibility is currently under investigation. Keywords: Alzheimer's disease, Cholinergic degeneration, Neurotoxicity
Processing of Frequent and Deviant Speech Stimuli as Revealed by LORETA

Laufer I.. and Pratt $\mathrm{H}$

Evoked Potentials Laboratory, Technion-Israel Institute of Technology, Haifa, Israel;

The mismatch negativity (MMN), which is elicited by deviance, was evoked in ten normal hearing, right handed native Hebrew speakers, whose age ranged between $19-30$ years.

Subjects had to discriminate between two V-C-V sequences (/aga/ and /ada/), which were auditory objects formed by fusion of speech elements, which differed in occurrence (standard and deviant).

Former research using either tones or complex sounds found that the sources of the mismatch magnetic field (MMF) are located bilaterally in the supratemporal planes, as well as in the right inferior parietal cortex.

The purpose of this study was to localize the MMN generators in the context of speech, using brain functional imaging (LORETAlow resolution functional tomography), which directly estimates the current density throughout the brain's activated areas using AEPs as input.

In agreement with former findings, results show that the MMN involves the activation of bilateral supratemporal planes, which tended to be more extensive in the right hemisphere. However, in the present study additional hemispheric differences in processing were found: activity in response to the frequent stimulus was more enhanced in the left supratemporal plane, whereas activity in response to the rare stimulus tended to be more enhanced in the right supratemporal plane.

These results corroborate other findings which suggest differential hemispheric processing of change detection, and a more prominent role of the right hemisphere in this process.

Keywords: Evoked potentials, Auditory object, Auditory cortex

On the role of feedback in learning with liquid neural networks

Legenstein R. ${ }^{1}$, Maass W. ${ }^{1}$ and Markram $\mathrm{H}^{2}$

Institute for Theoretical Computer Science, Technische Universität Graz; A-8010 Graz, Austria; ${ }^{2}$ Dept. of Neurobiology, Weizmann Institute for Science, Rehovot, 76100

The role and mechanisms of learning in recurrent neural microcircuits are still not well understood. In contrast to previous approaches based on attractor neural networks, we study learning paradigms in liquid neural networks which were recently introduced by Maass, Markram and Natschläger. The so-called liquid, a computer model for a neural microcircuit, is used to store information about past inputs in its high-dimensional dynamic state. Stable internal states are not required for a stable output, since readout-neurons can be trained to transform transient internal states into stable outputs. So far, the readout had been coupled with the liquid merely by feed-forward connections. We investigate several different architectures that make use of feedback connections from the readout into the liquid. In this variation of the model, information about past readout-output is available in the transient state of the liquid and may be used to stabilize the output. This yields a number of interesting improvements in the performance of the network after learning.

Keywords: Liquid computing; Recurrent neural networks; Learning

Completion effects in the human visual cortex

Lerner $Y^{1}$, Hendler $T^{2,3}$ and Malach $R$.

${ }^{7}$ Weizmann Institute of Science, Rehovot 76100 , Israel; ${ }^{2}$ Sourasky Medical Center, Tel Aviv 64239, Israel; ${ }^{3}$ Tel Aviv University, Tel Aviv, 69978

Human ability to recognize partially occluded objects is one of the most remarkable characteristics of the visual system. To what extent completion effects can be traced in the activity of specific areas within human visual cortex? Using fMRI, we studied completion related activation in high order object areas - i.e. lateral occipital complex (LOC). Subjects viewed three categories of images: a) intact line drawings of animals or abstract shapes ("whole"), b) the same shapes but occluded by parallel stripes that occupied about half of the images ("grid"), and c) the same visual parts as in b, but scrambled so that the relative position of regions between the stripes was replaced while the local feature structure remained intact ("scrambled"). Behavioral experiments revealed that subjects could successfully recognize the objects in the "grid" condition but not in the "scrambled" condition. fMRI data 
obtained from 11 subjects demonstrated higher activity in the LOC for the "whole" and "grid" conditions compared to the "scrambled" images (\% signal change: "whole" - $1.3 \pm 0.17$, "grid" $-1 \pm 0.14$, "scrambled" $-0.6 \pm 0.08$ ). Since the local features in both these conditions were identical the enhanced response obtained in LOC indicates the presence of non-local completion effects in this area. Furthermore, this pattern of activity was comparable to subjects' recognition performance. In contrast, early retinotopic areas responded similarly in both the "grid" and the "scrambled" conditions. Overall, these results point to the LOC as a central cortical area in which object completion effects are manifested.

Supported by Israel Academy 8009/00-1 and MP 6971 grants.

Keywords: Functional MRI, Object recognition, Visual areas, Occlusion.

\section{The cognitive effects of the apolipoprotein $\mathbf{E}$ genotype are elicited by environmental stimulation at a young age Levi O., Yakovlev G. and Michaelson D.M. \\ Dept. of Neurobiochemistry, Tel Aviv University, Tel Aviv}

A growing body of evidence suggests that the pathological effects of the allele E4 of apolipoprotein E (apoE), a key 4risk factor for Alzheimer's disease, are related to impairments in neuronal maintenance and repair. Recent studies indicate that apoE plays a critical role in brain development. The extents to which the roles of apoE in neurodegeneration and development are related and share common mechanisms is an enigma. In the present study we addressed these issues and examined whether cognitive development can be affected differentially by the different apoE genotypes. This was performed by subjecting 3 weeks' old mice transgenic for the human apoE isoforms apoE3 or apoE4 on a mouse null apoE background and control mice to an enriched environment for 10 weeks, and comparing, by means of T-tests, the extent to which this affected their learning and memory capabilities. This revealed that the learning ability of the controls and apoE3 transgenic mice increased markedly following exposure to the enriched environment, whereas that of the apoE4 was unaltered. Furthermore, the working memory (delayed match to sample on the T-maze) of the control and apoE3 transgenic mice also improved by experiencing the enriched environment, whereas that of the apoE4 transgenic mice was unaltered and similar to that of the non-treated apoE3 and control mice. The finding that cognitive development is affected by the apoE genotype provides a novel link between the developmental and neurodegenerative functions of apoE and suggests that the latter may be mediated by impairments in remodeling reconstructive processes.

Keywords: Alzheimer's disease, Apolipoprotein E, Learning and memory development

Correlation between blood-free testosterone concentration and sleep duration in healthy young men

Levinger $\mathrm{U}^{1}$, Gat $\mathrm{Y}^{2}$ and Gornish $\mathrm{M}$.

Depts. of Internal Medicine B, ${ }^{2}$ Gynecology and ${ }^{3}$ Radiology,

Rabin Medical Center, Petach Tigva, and Sackler School of

Medicine, Tel Aviv University, Tel Aviv

Background: Healthy young men who underwent varicocelle chemical embolisation, required less sleep. Testosterone, an androgenic anabolic steroid, affects the central nervous system. We measured serum free testosterone in a group of these patients, to determine whether there is a correlation with sleep duration

Methods: Thirty young men in general good health, underwent varicocelle chemical embolisation for infertility treatment. They were requested to complete a questionnaire regarding their sleep habits and duration of sleep. They were asked to fill in the questionnaire before treatment and again

3 months after start of treatment. Serum free testosterone was measured at the same times.

Results: In 30 young men (mean age $29 \pm 7$ ) serum free testosterone increased 3 months after the varicocelle procedure $(6.5 \pm 3.5$ vs. $11 \pm 3.1 ; \quad \mathrm{P}<0.05)$. Night sleep duration significantly decreased during the same period $(7.6 \pm 2.1$ vs $6.1 \pm$ $1.4 ; \mathrm{P}<0.05)$. Neither mood nor behavior patterns were altered in this group.
Conclusions: An increase in free serum testosterone concentration, following varicocelle chemical embolisation, was associated with a decreased need for sleep and a shortened sleep duration.

Keywords: Testosterone, Sleep, Varicocelle.

Gene profile and signal transduction events for neuroprotective and proapoptotic actions of 3,3epigallocatechin-3-gallate

Levites Y., Mandel S. and Youdim M.B.H.

Eve Topf and US National Parkinson's Foundation Centers of Excellence for Neurodegenerative diseases, Bruce Rappaport Family Research Institute and Depts. of Pharmacology, Faculty of Medicine, Technion, Haifa

Tea extracts have been previously reported to possess radical scavenger, iron chelating and anti-inflammatory properties in a variety of tissues. Recently we have shown that green tea extract and major green tea polyphenol, EGCG prevented MPTP-induced dopaminergic neuronal loss in mice. In the present work we extended this in vivo study to neuronal culture where two parkinsonism-inducing neurotoxins, 6-OHDA and $\mathrm{MPP}^{+}$were employed. Pretreatment for $15 \mathrm{~min}$ with EGCG $(0.1-5 \mu \mathrm{M})$ prevented $6-\mathrm{OHDA}(20 \mu \mathrm{M}$, from 40 to $70 \%$ cell survival) and $\mathrm{MPP}^{+}(400 \mu \mathrm{M}$ from 60 to $85 \%$ cell survival) induced human neuroblastoma (NB) SH-SY5Y cells death, whereas higher concentrations $(10-50 \mu \mathrm{M})$ lead to cell death. In order to elucidate genetic events in neuroprotection and neurodegeneration induced by EGCG, RNA from NB cells treated with EGCG $(1$ and $50 \mu \mathrm{M})$ was extracted, CDNA probes were synthesized and hybridized to a custom array consisting of 25 genes related to apoptosis and cell survival. EGCG $(1 \mu \mathrm{M})$ reduced the expression of $\mathrm{Bax}, \mathrm{mdm} 2$, TRAIL and 2 21 Waf1 mRNAs. However, a high, toxic concentration of EGCG $(50 \mu \mathrm{M})$ elevated the expression of Bax, Caspase 6, GADD 45, mdm2 and TRAIL mRNAs while Bcl-w was down-regulated. We further examined signal transduction events as possible mechanistic action of EGCG. The low, $1 \mu \mathrm{M}$ EGCG concentration did not affect the levels of phosphorylated ERK $_{1 / 2}$ by itself, but prevented their decrease induced by $6-$ OHDA. Similarly, this concentration did not change phosphorylated SAPK/JNK levels related to control, but did not manage to prevent the induction caused by 6-OHDA. However, a high EGCG concentration $(50 \mu \mathrm{M})$ exacerbated the effect of 6 OHDA on both MAPKS. This concentration range may reflect its respective therapeutic window in vivo. This study demonstrates that EGCG has concentration dependant neuroprotective and proapoptotic activity and these have been confirmed by gene expression analysis.

Keywords: EGCG, Neuroprotection, Apoptosis, Signal transduction

3,3-epigallocatechin-3-gallate attenuates neuronal cell death in 6-OHDA and MPTP models of Parkinson's disease: possible gene targets

Levites Y. , Mandel S. ${ }^{1}$, Maor $\mathrm{G}^{2}$ and Youdim M.B.H.

${ }^{T}$ Eve Topf and US National Parkinson's Foundation Centers of Excellence for Neurodegenerative diseases, Bruce Rappaport Family Research Institute and Depts of Pharmacology and Cell Biology ${ }^{2}$, Faculty of Medicine, Technion, Haifa

Oxidative stress is a contributing factor to the pathogenesis of neurodegenerative disorders such as Parkinson's and Alzheimer's diseases. Recently we have shown that tea extracts inhibit ironinduced lipid peroxidation of brain mitochondrial fraction as well as 6-hydroxydopamine-induced cell damage in neuronal cell lines and MPTP-induced dopaminergic neurons loss in vivo. In an attempt to elucidate the neuroprotective skills of major tea polyphenol, 3,3-epigallocatechine-3-gallate (EGCG) and possible gene targets for its action, the MPTP (N-methyl-4-phenyl-1,2,3,6tetrahydropyridine) model of Parkinson's disease was employed. C57-BL mice were treated with EGCG $(4-20 \mu \mathrm{mols} / \mathrm{kg} /$ day $/ 14$ days, orally). MPTP $(24 \mathrm{mg} / \mathrm{kg} /$ day, i.p.) was given for the last 5 days. 3 days after last injection, the mice were decapitated and brains were used for further investigations. Striatal Dopamine (DA), 3,4-dihydroxyphenylacetic acid (DOPAC) and homovanillic acid (HVA) content were determined by HPLC. MPTP caused a marked reduction in DA levels ( $40 \%$ of control). However, EGCG $(4 \mu \mathrm{mols} / \mathrm{kg} /$ day $/ 14$ days) conferred a significant protection against MPTP induced DA loss, as indicated by striatal dopamine and its metabolites content $(80 \%$ of control) as well as by Tyrosine hydroxilase (TH) content and activity and Superoxide dismutase (SOD) and Catalase activity. Gene expression profile induced by EGCG was analyzed using Atlas mouse cDNA 
expression array. The expression of number of different genes involved in signal transduction pathways leading to neuronal survival, such as c-jun, CREBP1, MAP3K, MEKK $1 / 2$, STAT1, IGF1R was up regulated. This study is the first to demonstrate neuroprotective activities of EGCG as well as a detailed profile of simultaneous gene changes in brain as a result of EGCG consumption.

Keywords: EGCG, Neuroprotection, Parkinson's disease

Conformational polymorphism of wild-type and mutant prion proteins: A molecular dynamic study Levy Y. and Becker O.

Dept. of Chemical Physics, School of Chemistry, Tel Aviv

University, Tel Aviv 69978

Conformational transitions are thought to be the prime mechanism of prion diseases. In this study the dynamics of a wild-type prion protein (PrP), as well as the D178N and E200K mutant proteins, were explored, enabling the characterization of the normal isoforms $\left(\mathrm{PrP}^{\mathrm{C}}\right)$ and partially unfolded isoforms $\left(\mathrm{PrP}^{\mathrm{PU}}\right)$ of the three prion protein analogs. It was found that the three PrP analogs differ in three respects: (1.) The relative stability of the $\operatorname{PrP}^{\mathrm{C}}$ and the $\mathrm{PrP}^{\mathrm{PU}}$ states, (2.) the transition pathways from $\mathrm{PrP}^{\mathrm{C}}$ to $\mathrm{PrP}^{\mathrm{PU}}$, and (3.) the relative stability of the three helices in the $\operatorname{PrP}^{C}$ state. In particular it was found that while helix 1 (residues 144-156) is the most stable helix in wild-type PrP, its stability is dramatically reduced by both mutations. This destabilization is due to changes in the charge distribution that affects the internal salt-bridges responsible for the greater stability of this helix in wild-type PrP. Though both mutations result in similar destabilization of helix 1 , they a have different effect on the overall stability of $\operatorname{PrP}^{\mathrm{C}}$ and of $\operatorname{PrP}^{\mathrm{PU}}$ isoforms and on structural properties. The destabilization of helix 1 by mutations provides additional evidences to the role of this helix in the pathogenic transition from the $\left(\operatorname{PrP}^{c}\right)$ to the pathogenic isoform $\left(\mathrm{PrP}^{\mathrm{Se}}\right)$.

Keywords: Prion proteins, Conformational transition, D178N and E200K

\section{Identification of determinants in a cytosolic domain of L-type $\mathrm{Ca}^{2+}$ channel responsible for channel kinetics} Livneh A. and Atlas D.

Dept. of Biological Chemistry, Hebrew University of Jerusalem, Jerusalem 91904

Influx of $\mathrm{Ca}^{2+}$ through voltage gated calcium channels is necessary for release of neurotransmitter from nerve endings. The kinetic properties of these channels determine the amount of $\mathrm{Ca}^{2+}$ ions entering the cell, and thus determine the amount of transmitter released. We used Xenopus laevis oocytes as a heterologous expression system for mRNAs of mutated calcium channel $\alpha 1$ subunit, and measured the kinetic properties of inward currents. We show that replacing negatively charged motif in a cytosolic domain of the $\mathrm{Ca}_{\mathrm{v}} 1.2$ subunit changes the kinetic properties of the cardiac L-type channel. The mutated channel display kinetic properties similar to neuronal channels. We discuss the possible role of this motif in channel kinetics, and its physiological and structural implications.

Keywords: Calcium channel, Xenopus oocytes, Activation rate, Inactivation rate

\section{Synaptic information efficacy: bridging the gap between} biophysics and function

London M. ${ }^{1}$, Shraibman A. ${ }^{2}$ and Segev I. ${ }^{1}$

Dept. of Neurobiology and Center for neural computation ${ }^{\prime}$, Dept of Computer Science ${ }^{2}$, Hebrew University, Jerusalem

We provide a new functional measure, termed the "synaptic information efficacy" SIE, to assess the impact of synaptic input on spike output. SIE is the mutual information (in bits/s) shared by the pre-synaptic input and post-synaptic output spike trains. To estimate SIE we use a novel method based on a modern compression algorithm. This method detects the effect of a single synaptic input on the post-synaptic spike output, in the presence of massive background synaptic activity in neuron models of progressively increased realism. SIE increases both with the increase in time-locking between the input synapse activity and the output spike, as well as with increase in the average number of output spikes initiated by the synapse, and it is critically dependent on the "context" within which the synapse operates. Systematic exploration of the effect of the synaptic and dendritic parameters, and the statistics of the background input on the SIE provides a fresh look at the synapse as a communication device and furnishes a novel quantitative measure concerning "how much the dendritic synapse informs the axon".

Supported by the BSF and the Israel Science Foundation Key words: Synaptic efficacy, Dendritic processing, Information Theory

\section{Modulation of complement-receptor-3 (CR3/MAC-1)} mediated myelin phagocytosis

Makranz C., Slobodov U., Reichert F. and Rotshenker S.

Dept. of Anatomy \& Cell Biology, Hebrew University Faculty of Medicine, Jerusalem

The removal of damaged myelin is central to repair after injury to axons and in autoimmune demyelinating diseases. Myelin contains molecules that inhibit the growth of adult axons. The removal of injury-produced degenerating myelin eliminates myelin-related inhibition of regeneration. In autoimmune demyelinating diseases, myelin that was first damaged by autoimmune mechanisms can activate the complement system, which leads to the formation of membrane attack complexes that further disrupt myelin. The removal of autoimmune-damaged myelin may slow down this vicious circle where already damaged myelin leads to the further disruption of intact myelin. Macrophages and microglia remove myelin by phagocytosis. We have previously suggested that macrophages and microglia may be present at different functional/activation states that determine the efficiency by which they phagocytose myelin, and further documented that macrophages that were exposed to different inflammatory environmental conditions differed in their capacity to phagocytose and degrade myelin. We have previously suggested that the modulation of myelin phagocytosis may be carried out through CR3/MAC-1, which mediates and dominates receptor-mediated myelin phagocytosis. We presently document that CR3/MAC-1mediated myelin phagocytosis is subject to modulation by a variety of mechanisms. First, by cytokines GM-CSF and M-CSF, which upregulate cell surface expression of CR3/MAC-1. Second, by complement, which augments CR3/MAC-1-mediated myelin phagocytosis. Third, by extracellular ligands, which produce conformational changes in CR3/MAC-1; some inhibit and other augment CR3/MAC-1-mediated myelin phagocytosis. Fourth, by altering intracellular cAMP levels, which modulates CR3/MAC-1mediated myelin phagocytosis.

Keywords: Wallerian-degeneration, Regeneration, Demyelination, Macrophage, Microglia

Mother-infant interaction in rats lacking the $\mathrm{CCK}_{\mathrm{A}}$ receptor. Malkesman O. ${ }^{1,2}$, Avnon Y.L. ${ }^{1}$, Hurwitz I. ${ }^{2}$ and Weller A ${ }^{T}$ Dept. of Psychology and ${ }^{2}$ Interdisciplinary Program in the Brain Sciences, Bar Ilan University, Ramat-Gan, Israel.

Stuuies have shown that the peptide cholecystokinin (CCK) is involved in mother- infant interaction through the activation of $\mathrm{CCK}_{\mathrm{A}}$ receptors. The Otsuka Long Evans Tokushima Fatty (OLETF) rat lacks functional $\mathrm{CCK}_{\mathrm{A}}$ receptors because of a genetic abnormality. In this study, we investigated whether this abnormality affects the infant's and/or the mother's behavioral characteristics during interaction. 10 OLETF dams and 10 control (Long Evans Tokushima LETO) dams were observed during a 10 min interaction. Each dam interacted separately with one OLETF and one LETO pup. Infant ultrasonic vocalizations (USV) and maternal behaviors were counted

We found that OLETF pups emitted more ultrasonic vocalizations, received more body and anogenital licking, and were carried in mouth by the dam more than controls. In addition, OLETF dams carried pups less frequently, but nursed the pups more than the control dams. In accordance with previous findings (Weller \& Dubson, Pharmacol Biochem Behav 59: 843-851 [1998]), these results suggest that OLETF pups, like normal pups given a selective $\mathrm{CCK}_{\mathrm{A}}$ antagonist, elicit and obtain more maternal care from the dam. Therefore, activation of $\mathrm{CCK}_{\mathrm{A}}$ receptor may reduce infant's need for contact and interaction.

Supported by the Israel Science Foundation

Keywords: Maternal behavior, Mother-infant interaction, USV, $\mathrm{CCK}_{\mathrm{A}}$ receptors. 
Early gene expression changes in MPTP model of Parkinson's

Mandel S. ${ }^{1}$, Maor G. ${ }^{2}$ and Youdim M.B.H. ${ }^{1}$

'Eve Topf and US National Parkinson's Foundation Centers of Excellence for Neurodegenerative Diseases, Bruce Rappaport Family Research Institute and Dept.s of Pharmacology and ${ }^{2}$ Cell Biology, Faculty of Medicine, Technion, Haifa

MPTP-induced dopaminergic cell death in mice, human and nonhuman primates is considered a model most closely resembling idiopathic Parkinson's disease. Recently, we reported specific nigro-striatal gene profile changes in the chronic 5 days MPTP model, in the late stage of degeneration, employing cDNA expression array. As an attempt to elucidate the early (3-24 hr) cascade of events in MPTP-induced cell death, time dependent gene expression profile of acute MPTP treated mice was investigated by means of quantitative real-time RT-PCR and ininvestigated by means of quantitative real-time RT-PCR and in-
situ hybridization. The expressions of cyclin B2, N-methyl-Daspartate (NMDA2A), IL-1 3 , glutathione peroxidase, GDNF and glutathione reductase mRNAs, were maximally induced already three hours after exposure to MPTP and then they declined to control levels or even below it, except for glutathione peroxidase and GDNF, whose expressions remained high along a period of twenty-four hours and 5 days, respectively. An elevation in the expression of IL-10, IL-1 R type II, parkin and oxidative stressinduced protein, A170 mRNAs, begin to manifest six hours after MPTP exposure. Glutathione-S-transferase and NADPH cytochrome P450 mRNAs induction, became evident only twenty-four hours after MPTP injection. Tyrosine hydroxylase (TH) mRNA alterations strictly correlated with TH protein changes in the substantia nigra. Immunoreactivity of TH along the initial $24 \mathrm{~h}$ MPTP treatment period, showed no dopaminergic cell loss in the SN. This finding strengthens the importance of investigating the initial gene expression changes induced by acute MPTP dosage, in contrast to the chronic treatment, where gene expression is examined only at the time most neurons have died.

Keywords: MPTP, Microarray, Neuroprotection, Parkinson's disease

\section{Period doubling of calcium spike firing in a model of a}

Purkinje cell dendrite

Mandelblat Y., Etzion Y., Grossman Y. and Golomb D.

Zlotowski Center for Neuroscience and Dept. of Physiology, Ben-

Gurion University, Beer-Sheva 84105.

Recordings from cerebellar Purkinje cell dendrites have revealed that in response to sustained current injection, the cell firing pattern can move from tonic firing of $\mathrm{Ca}^{2+}$ spikes to doublet firing and even to quadruplets or more complex firing. Blockade of $\mathrm{Na}$ currents doesn't change these firing patterns substantially. We show that the experimental results can be viewed as a slow transition of the neuronal dynamics through a period-doubling (PD) bifurcation. We develop and study a simple, onecompartment model of Purkinje cell dendrite. The effects of parameters such as temperature, applied current and potassium reversal potential in the model resemble their effects in experiments. The model dynamics involve three time scales. $\mathrm{Ca}^{2+}$. dependent $\mathrm{K}^{+}$currents, with intermediate time scales, and a very slow hyperpolarizing current (Iy). Fast-slow analysis of the neuronal dynamics, with the activation variable of ly considered as a parameter, reveals that the transitions occurs via a cascade of PD bifurcations of the fast-and-intermediate subsystem as this slow variable increases. We carry out another analysis, with the $\mathrm{Ca}^{2+}$ concentration considered as a parameter, to investigate the conditions for the generation of doublet firing in systems of this type. We find that PD in these systems can occur only if:

1. The time scale of the intermediate variable (here, the decay rate of the calcium concentration) is slow enough in comparison with the inter-spike interval of the tonic firing at the transition, but is not too slow;

2 . There is a bistability of the fast subsystem of the spike generating variables.

Keywords: Purkinje cell, Dendrite, Doublet firing, Period doubling

Synaptic depression mediates bistability of neuronal networks. Manor Y. ${ }^{1}$ and Nadim F.

${ }^{7}$ Life Sciences Dept. , Ben-Gurion University of the Negev, Beer

Sheva; ${ }^{2}$ Biology Dept., Rutgers University.

Synaptic depression is a common dynamical property observed in central synapses. We show that when synaptic depression is incorporated in reciprocally inhibitory loops, bistability in network function can emerge. Experiments were performed by coupling biological pacemaker neuron to a model neuron using artificial inhibitory depressing synapses. This hybrid biological-model network exhibited two modes of oscillation, one where the oscillation frequency was determined solely by the intrinsic properties of the biological pacemaker and the other where the frequency was largely affected by the dynamics of the depressing synapses. These two modes of oscillation were often present in the same parameter range to produce bistability. The activity of the network could be switched from one mode to the other by engaging a regenerative process that increased, or decreased, the strength of both synapses. In the bistable regime, this process could be triggered, for example, by a brief current pulse injected in either cell. This mechanism suggests that, in rhythmically active systems, network reconfiguration can occur by allowing depressed synapses to recover from or decay to their depressed state.

Keywords: Synaptic depression, Bistability, Reciprocal inhibition, Dynamic clamp.

BDNF occludes tetanic stimulation induced-LTP in the rat dentate gyrus in vivo-electrophysiological and molecular

characterization.
Maroun M.,3 ${ }^{1,3}$, Dumas S. ${ }^{2}$, Mallet J. ${ }^{2}$ and Laroche S. ${ }^{1}$

ILaboratoire de Neurobiologie de l'Apprentissage, de la memoire et de la Communication, CNRS UMR 8620, Universite Paris Sud, 91405 Orsay, France; 2Laboratoire de Genetique Moleculaire de la Neurotransmission et des Processus Neurodegeneratifs, CNRS

UMR C9923, Hopital de la Pitie Salpetriere, 75013 Paris,

France; 3 Present address: Dept. of Psychology, University of Haifa, Haifa 31905

Brain-derived neurotrophic factor (BDNF) has been reported to induce long-lasting potentiation of synaptic efficacy in dentate gyrus (DG) neurons of the rat hippocampus in vitro and in vivo. There is some evidence to suggest that the mechanisms underlying BDNF-induced potentiation share some similarities with those that underlie long-term potentiation (LTP). In LTP, one key mechanism is the activation of genes and de novo synthesis of proteins.

In the present study, we investigated whether BDNF-induced potentiation in the DG occludes LTP produced by tetanic stimulation and examined the molecular mechanisms underlying BDNF-induced potentiation.

The results show that intrahippocampal infusion of BDNF $(2 \mu \mathrm{M})$ induced a significant and long-lasting potentiation of the EPSP recorded in the DG in response to perforant path (PP) stimulation, compared to controls. Furthermore, prior infusion of BDNF occluded further potentiation in dentate granule cells produced by tetanic stimulation of the PP, suggesting that both processes share, at least in part, common underlying mechanisms.

Using in situ hybridization, we are currently analyzing the molecular basis of BDNF-induced potentiation. We are investigating whether BDNF induces transcription of the immediate early genes (IEGs) Zif268 and Arc, and of the mRNA encoding CaMKII. These three genes have been shown to be differentially regulated in LTP

The results from this project will shed light on the molecular mechanisms mediating the late phases of BDNF-induced potentiation and of tetanic stimulation-induced LTP in the DG in vivo.

Keywords: BDNF, LTP, Plasticity, Genes

High B value Q-space analyzed diffusion: a new method of brain imaging following demyelination in Alzheimers and vascular dementia

Mayzel-Oreg O. ${ }^{1}$, Assaf Y. ${ }^{1,2}$, Gigi A. ${ }^{2}$, Ben-Bashat D. ${ }^{2}$

Verchovsky R ${ }^{3}$, Mordohovitch M. ${ }^{3}$, Graif M. ${ }^{2}$, Rider-Groswasser $\mathrm{I}^{3}$, , Hendler T. ${ }^{2}$, Cohen Y. ${ }^{1}$ and Korczyn A.D. ${ }^{3,4}$

${ }^{i}$ School of Chemistry, Tel-Aviv University, Tel-Aviv 69978, Israel;

${ }^{2}$ Wohl Institute for Advanced Imaging and ${ }^{3}$ Dept. of Neurology Tel Aviv Sourasky Medical Center, Tel Aviv, 64239, Israel; ${ }^{4}$ Sackler Faculty of Medicine, Tel viv University, Tel Aviv 69978

Diffusion imaging at high $b$ values was shown to be highly sensitive to white matter (WM) pathology (Assaf et al, MRM 2001 , accepted for publication). Diffusion images, when analyzed using the q-space approach, show areas of abnormal white matter in multiple sclerosis (MS) not detected by other MRI methods like $\mathrm{T}_{2^{-}}, \mathrm{T}_{1}$-weighted imaging, FLAIR, MTR and DTI.

High $b$ value q-space analyzed diffusion MR images acquired on human subjects with vascular dementia (VaD) showed massive WM loss. These areas of WM tissue loss were not detected with 
the other imaging methods. In areas of hyperintense signal in the FLAIR and $\mathrm{T}_{2}$-weighted images, there was a marked reduction in WM density as observed by the high b value, q-space analyzed images, suggesting tissue degeneration in those areas.

High b value q-space analysis also appears to be sensitive to WM changes in images acquired on human subjects with Alzheimer disease $(\mathrm{AD})$, especially in frontal white matter areas. Again, these areas of WM loss observed with q-space analysis were much less apparent using other imaging modalities.

To summarize, the high $b$ value $q$-space diffusion MRI is apparently highly sensitive to WM changes and may be useful in the estimation of tissue loss in $\mathrm{VaD}$ and $\mathrm{AD}$. This method might contribute to the discrimination between different WM pathologies such as $\mathrm{VaD}$ and $\mathrm{AD}$.

Keywords: Alzheimer, Vascular dementia, q-Space, Diffusion MRI.

\section{Dexanabinol is neuroprotective in the MPTP model of Parkinson's disease \\ Meilin S., Bar-Joseph A., Lavie V., Grienstein Y. and Fink G. Pharmos Ltd, Kiryat Weizmann, Rehovot 76326}

Parkinson's disease (PD) is a neurodegenerative disorder characterized by reduction in striatal dopamine (DA) content caused by the loss of dopaminergic neurons in the Substantia Nigra pars compacta (SNpc) and their projections to the striatum. In the present study we investigated whether Dexanabinol (dex), a non-psychotropic cannabinoid and non-competitive NMDA receptor antagonist, with anti-oxidant and anti-inflammatory activities, has a protective effect on central dopaminergic neurons from MPTP toxicity. Mice were treated with MPTP or saline IP. Mice were injected IP with either Dexanabinol $(10,20,30 \mathrm{mg} / \mathrm{kg}$ or twice with $20 \mathrm{mg} / \mathrm{kg}$ at 24 hours interval) or its vehicle $(5 \mathrm{ml} / \mathrm{kg})$ prior to the first MPTP administration. The animals were followed for body weight seven days after which their brains were removed, cryosectioned $(20 \mu \mathrm{m})$ at the levels of the $\mathrm{SN}$ and the striatum and immunohistochemically labeled by rabbit anti mouse tyrosine hydroxylase (TH)(1:100 Calbiochem). The MPTP-treated (dex treated animals and non treated animals) animals lost about $10 \%$ of their body weight within the first $24 \mathrm{~h}$ after administration. However the body weight of the non-treated animals remained low $(\mathrm{p}<0.05)$ whereas the dex treated group gain weight. $\mathrm{TH}$ labeling revealed a $60 \%$ decrease $(p<0.05)$ in the number of TH immunoreactive neurons in the SNpc following MPTP administration. Dexanabinol $20 \mathrm{mg} / \mathrm{kg}$ increased by $25-30 \%$ the number of TH positive cells compared to vehicle $(p<0.05)$. These results suggest that Dexanabinol is neuroprotective in MPTPinduced PD model.

Keywords: Parkinson's Disease, MPTP, Dexanabinol

Dominant-negative synthesis suppression of $\mathrm{Cav} 2.2$ channels, induced by truncated constructs.

Meir A. ${ }^{1}$, Bertaso F., Raghib A., Davis A., Page K.M. and Dolphin A.C.

Dept. of Pharmacology, University College London, Gower Street, London, WCIE 6BT: UK. 'New address: Alomone labs P. O. Box 4287, Jerusalem 91042

Voltage-gated calcium $\left(\mathrm{Ca}_{\mathrm{V}}\right)$ channels play a major role in the normal functioning and pathophysiology of neurons and other excitable cells. Ca $a_{y}$ channels consist of a four transmembrane domains (each composed of $6 \alpha$-helixes) protein $(\alpha 1)$ and auxiliary subunits. Alternative splicing and mutations of the $\alpha 1$ subunit often result in transcription and translation of partial channels. In purification studies of $\mathrm{Ca}_{\mathrm{v}} 2.1$ and $\mathrm{Ca}_{\mathrm{v}} 2.2$, a substantial protein band of about $95 \mathrm{kD}$ was observed, which was identified as the first two domains of $\mathrm{Ca}_{\mathrm{v}} 2.1$. In Episodic Ataxia 2 (EA2), truncation mutations occur after the end of Domain that substantial portions of the Cav 2.1 channel may be synthesized and inserted into the plasma membrane.

In order to examine the effect of such proteins, we have constructed hemi-channel proteins (Domain ${ }_{\text {I-II }}$ and Domain ${ }_{\text {III-IV }}$ of $\mathrm{Ca}_{\mathrm{y}} 2.2$ ) and co-expressed them in COS-7 cells either alone or together with full-length $\mathrm{Ca}_{\mathrm{v}} 2.2$ and auxiliary subunits $(\alpha 2-\delta 1$ and $\beta 4$ ). In addition to electrophysiological measurements (whole cell and cell attached), we used Green Fluorescent Protein (GFP) tagged channels to examine the level of expression. Co-expression of the two complementary hemi-channels resulted in fully functional channels with low level of expression. Co-expression of
Domain $_{\mathrm{I}-\mathrm{II}}$ with the full-length $\mathrm{Ca}_{\mathrm{V}} 2.2$ resulted in large inhibition of the whole cell Cay 2.2 current densities and of the level of detected GFP. However, the single channel properties of $\mathrm{Ca}_{\mathrm{v}} 2.2$ under these conditions were unchanged.

Together these results indicate that $\mathrm{Ca}_{\mathrm{y}}$ related proteins inhibit the membrane insertion of the full-length channel but have no effect on the channel properties once expressed in the membrane Further biochemical data indicate that synthesis of the full-length channel is suppressed by constructs containing Domain

Keywords: Calcium, Channel, Cav, Truncation, Domain

Molecular correlates of plasticity in a one-day-old chick as revealed by differential display.

Meiri N., Adelheit S., Radoul M.

Institute of Animal Science, Agriculture Research Organization The Volcani Center, Bet Dagan 50250 Israel.

Long-term storage of information, whether, memory, habituation, sensitization or accommodation to changing environment, is thought to be subserved by functional and/or structural remodeling of neuronal circuits. This work comes to answer two questions:

1. What are the molecular pathways involved in memory storage? 2. Are the pathways involved, common to both declarative and non declarative memory?

Since memory formation is expected to involve structural changes in network organization, the ideal model for identifying the involved genes will focus on a behavioral paradigm that emphasizes growth and development, where structural changes and hence the involved genes might be amplified. The day-oldchick declarative passive avoidance, and non-declarative thermal conditioning behavior paradigms, are used here to identify the aforementioned molecular changes. In a passive avoidance learning paradigm a chick learns to avoid a bead coated with an unpleasant tasting substrate. The intermediate medial hyperstriatum ventrale (IMHV) is centrally involved in storing this memory. Memory for temperature accommodation is neuroanatomicaly located in the anterior hypothalamus preoptic area $(\mathrm{AH} / \mathrm{PO})$

After either passive avoidance training or thermal conditioning the IMHV or $\mathrm{AH} / \mathrm{PO}$ respectively are dissected in a time course ranging from minutes to 24 hours and changes in mRNA induction is monitored using mRNA fingerprinting and differential display. Preliminary screening of about $20 \%$ of IMHV and AH/PO mRNA from passive-avoidance trained chicks and thermal conditioning revealed a clear induction of 24 genes which are being analyzed. Among the induced genes are 14-3-3, N-CAM and HSP90 which have been implicated with both growth and memory.

Keywords: Memory, Passive avoidance, Thermotolerance, chicks, Differential display.

\section{Memory in liquid neural networks}

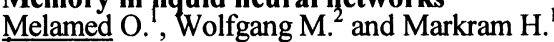

${ }^{7}$ Dept. of Neurobiology, Weizmann Institute for Science, Rehovot 76100, Israel; ${ }^{2}$ Institute for Theoretical Computer Science, Technische Universität Graz; A-8010 Graz, Austria

A key challenge is to understand memory storage and retrieval in neural microcircuits while an organism is engaging a rapidly changing environment. Current models propose that perceptions lie within a particular pattern of electrical activity, which requires specific modifications in the microcircuitry to store and retrieve memories. In the framework of a new theory of information processing in recurrent neural networks, termed "liquid computing", all memories are potentially available at any moment in time within the current state of activity of the network. The plasticity of the microcircuitry is required to improve the capability of the network to maintain past inputs in the current state, rather than to store any specific memory. In this model, the input as a function of time results in complex perturbations in the activity of a recurrent neural network, much like the effect of sound waves on the surface of a liquid. These perturbations represent an analogue environment in which past inputs are integrated across multiple time scales and from which readout elements can learn to extract information at different moments back in time. We used a recurrent network to represent the liquid 
and a parallel set of neurons to represent the readout element. The liquid was modified in terms of synaptic properties, connectivity structure and in terms of the number and types of neurons. Spike train inputs with different temporal structures were used as environmental stimuli to the liquid and the readout was trained to retrieve past activity patterns using only the current state of activity. The study demonstrates the capability of recurrent neural networks to provide a liquid environment that maintains information of past inputs for subsequent real-time memory retrieval.

Keywords: Liquid computing; Recurrent neural networks; Memory storage and retrieval.

\section{Particle tracking in recurrent neural networks: a method for} measuring dynamical properties

Melamed O. and Markram H.

Dept. of Neurobiology, Weizmann Institute for Science, Rehovot, 76100

Neural networks provide a powerful method to explore dynamic activity patterns that may emerge in the nervous system. A general approach to measure, characterize, and analyze the potential dynamics of a recurrent neural network is however, still missing. We show that by tracking the probability of finding a "test particle" in any one neuron, a particle distribution can be obtained, which provides the necessary information to derive the dynamical properties of an active recurrent neural network. We show that a spectrum of dynamic properties including the number, location and structure of attractor states, measures of contribution of a single neuron to the dynamic activity state of the entire network, structure of intersecting recurrent sub-loops, as well as various physical measures such as entropy and "kinetic energy" of the network. Network particle distributions can also be used in learning algorithms to engineer specific neural networks.

Keywords: Recurrent neural networks; Dynamical properties; Particle probabilities

\section{TNF alpha decreases learning capacity and NGF expression in}

Mendelson . $^{3}$, Levav T. ${ }^{1}$, Green T. ${ }^{1}$, Huleihel $\mathrm{M}^{2}$ and Golan

Depts. of Developmental Molecular Genetics ${ }^{I}$ and Immunology ${ }^{2}$, Faculty of Health Sciences and Zlotowski Center for Neuroscience, Ben-Gurion University, Beer-Sheva

TNF- $\alpha$ and other cytokines, which are polypeptide growth factors, produced mainly by and involved in the regulation of immune response. Recently, cytokines were demonstrated in the central nervous system (CNS) and were shown to control neuronal functions. Exposure of brain slices to extrinsic TNF $\alpha$ attenuated the induction of synaptic plasticity in different regions of the hippocampus. This may be directly affected by TNF- $\alpha$ or mediated through other factors. To study the involvement of TNF $\alpha$ in neuronal functions we have examined developmental and behavioral aspects in the TNF $\alpha \quad$ knok-out (TNF $\alpha-\mathrm{KO}$ ) mice compared with wild type (wt). TNF $\alpha-\mathrm{KO}$ mice showed significant and specific advantage in performance of spatial learning compared to $\mathrm{wt}$, as examined in the Morris water maze (32.2 sec. and $20.4 \mathrm{sec}$. respectively, in the correct quarter). No significant differences were observed in the performance of both study groups of mice in exploratory behavior, activity, anxiety, or motor coordination. Examination of nerve growth factor (NGF) levels in the mice brains following the accomplishment of the probe test show increased levels of NGF in the TNF-KO mice hippocampi as compared to the wt $(70$ and $28 \mathrm{ng} / \mathrm{mg}$. prot. respectively). This difference was specific to the hippocampus region, since no difference in NGF levels at the frontal cortex, were observed between the $\mathrm{KO}$ and wt mice $(76$ and $89 \mathrm{ng} / \mathrm{mg}$. protein). To explore the possibility that elevated levels of NGF represent developmental difference between both groups of mice, we have examined NGF levels at postnatal days $(2,7,14$ and 21$)$. Higher levels of NGF were detected in TNF-KO mice as compared to wt newboris in all examined days. Accordingly, we suggest that TNF $\alpha$ may control neuronal development and function; this effect could be mediated through NGF. Thus, TNF $\alpha$ may be important in brain regions where NGF is a key molecule for accurate circuite formation and function.

Keywords: TNF $\alpha$, NGF, Learning and memory, Development.
Studying architecture of $\mathbf{G}$ protein coupled $\mathbf{K}^{+}$channels using fluorescence based approaches

Meydani I., Kalmanzon E. and Reuveny E.

Dept. of Biological Chemistry, Weizmann Institute of Science, Rehovot

G-protein-activated potassium channels (GIRK) belong to the large superfamily of the inwardly rectifying potassium channels. Members of this family form functional tetramers, in which GIRK1 and GIRK4 channel subunits are linked in alternating positions. The activation of GIRK channels is mainly mediated by the free $G \exists$ ( subunits of the $G$ protein, through a direct interaction with the cytosolic $\mathrm{C}$ and $\mathrm{N}$ termini of the channel. It is not known how the channel $\mathrm{N}$ - and $\mathrm{C}$-termini cytoplasmic domains are oriented relative to each other and whether these domains undergo conformational changes during channel gating. In the present study we use the fluorescence resonance energy transfer (FRET) technique to elucidate the relative orientation of the cytoplasmic domains. We fused either the $\mathrm{N}$ - or the C-termini of GIRK 1 and GIRK 4 with CFP or YFP at various combinations and expressed them in either HEK293 cells for FRET measurements or in Xenopus oocytes for functional assay. We found various degrees of FRET efficiency depending on the fusion position, suggesting a specific architecture for assembly. In addition, these fused channel proteins had various degrees of gating capabilities. We conclude that using FRET it is possible to determine the orientation of membrane protein subunits assembly.

Functional implications of the direct interactions of the voltage-gated potassium channel Kv2.1 with syntaxin $1 \mathrm{~A}$ and SNAP-25

Michaelevski I. and Lotan I.

Dept. of Physiology and Pharmacology, Sackler School of Medicine, Tel-Aviv University, Tel Aviv

Previously, we identified direct interaction of both $\alpha$ and $\beta$ subunits of the $\mathrm{Kv} 1.1 / \mathrm{Kv} \beta 1.1$ voltage-gated potassium channel with syntaxin $1 \mathrm{~A}$ and SNAP-25. Further, we characterized the functional implication of the interaction of syntaxin with the channel in Xenopus oocytes and showed that syntaxin 1A increases the extent of inactivation and affects biphasically the current amplitude (Fili et al., 2001). Recently, we found that another voltage-gated potassium channel, belonging to a different subfamily, Kv2.1, interacts physically with syntaxin $1 \mathrm{~A}$ and SNAP-25 in oocytes and in vitro. Here we describe the functional implication of the interaction of syntaxin $1 \mathrm{~A}$ with $\mathrm{Kv} 2.1$ channel in Xenopus oocytes. Syntaxin 1A did not affect the current
amplitude of $\mathrm{Kv} 2.1$, in contrast to $\mathrm{Kv} 1.1 / \mathrm{Kv} \beta 1.1$, but affected kinetic parameters of the C-type inactivation of the current. Syntaxin 1A enhanced the entrance to and slowed down the recovery from inactivation. Currently, we characterize the functional effects of the interaction of SNAP-25 with the channel and the interplay between the syntaxin and SNAP-25 modulations of the channels.

Keywords: Kv channel, Syntaxin, SNARE complex, Gby.

Spike driven synaptic dynamics -- a self-saturating Hebbian paradigm

Mongillo G. ${ }^{1}$ and Amit D.J. ${ }^{1,2}$

${ }^{T}$ Racah Institute of Physics, Hebrew University, Jerusalem, Israel:

${ }^{2}$ Dept. of Physics, University of Rome "La Sapienza", Rome, Italy

A detailed spike-driven synaptic dynamics is simulated in a large network of spiking neurons, implementing the full double dynamics of neurons and synapses. The repeated presentation of a set of external stimuli is shown to structure the network to the point of sustaining Working. Memory (celective delay activity). When the synaptic dynamics is analyzed as a function of pre- and post-synaptic spike rates in functionally defined populations, it reveals a novel variation of the Hebbian plasticity paradigm: In any functional set of synapses (between pairs of neurons stimulated-stimulated; stimulated-delay; stimulated-spontaneous etc...) there is a finite probability of potentiation as well as of depression. This leads to a saturation of potentiation or depression at the level of the ratio of the two probabilities. When one of the two is much larger than the other, the familiar Hebbian mechanism is recovered. But where correlated working memory is formed, it prevents over-learning. Constraints relevant to both the stability of the acquired synaptic structure and the regimes of global activity allowing for structuring, are expressed in terms of the parameters describing the single-synapse dynamics.

The synaptic dynamics is discussed in the light of experiments observing precise spike timing effects and related issues of biological plausibility.

Keywords: synaptic plasticity, Hebbian learning, Spiking neurons, Working Memory. 
Intracellular evidence for diverse functional role of inhibition in the primary visual cortex

Monier C., Chavane F., Baudot P., Borg-Graham L. and FregnacY. Unite de Neurosciences Integratives et Computationnelles, UPR CNRS 2191, Gif-sur-Yvette, France

In the primary visual cortex, most cells respond selectively to the orientation of edges and their direction of motion. Evidence for the presence of inhibitory input in non-preferred orientation is contradictory. We thus decided to quantitatively readdress the respective contribution of excitatory and inhibitory input using intracellular recordings of visual cortical cells. Tuning of subthreshold responses to moving light bars was established by separating depolarizing and hyperpolarizing components and trialto-trial variability of the membrane potential was measured to distinguish between no or weak inputs and silent shunting inhibition. In patch recordings under voltage clamp, a decomposition method was applied to extract the excitatory and inhibitory conductance tunings. Results show that optimal orientations of significant hyperpolarizing component are observed in majority for non-preferred orientations. We can distinguish between two situations, when the dominant direction of excitatory input is in the preferred direction and when it is not. In the first case, the inhibitory tuning is in the preferred, crossoriented or in the opposed direction. In the second case the cell presents excitatory tuning selectivity for orientation/direction but not in the preferred direction of the spike rate and inhibitory tuning has the same optimal direction as excitatory input. In addition, in some examples we show that large conductance increase was concomitant with a reduction of trial-to-trial variability. In conclusion, inhibition seems to play diverse computational role, critical in cases where the inhibition shapes directly the orientation or the direction selectivity.

Keywords: in vivo intracellular recordings, Visual cortex, Inhibition

High Dehydroepiandrosterone levels decrease the effect of electronvulsive shock (ECS) in an animal model of depression Morad O. ${ }^{1,2}$, Yadid G. ${ }^{2}$, Weizman A. and Maayan R.

${ }^{T} F M R C$, Faculty of medicine, Tel -Aviv University, Tel Aviv; ${ }^{2}$

Fculty of Life Sciences, Bar Ilan University Ramat Gan

Dehydroepiandrosterone (DHEA) and dehydroepiandrosterone sulfate (DHEAS) are neuroactive steroids which are synthesized in the brain and display allosteric antagonistic properties at GABAA receptor mediating behavioral changes. In a previous study, we found that high basal levels of DHEAS can predict a lack or low response to electroconvulsive therapy (ECT) in depressive patients (Maayan et al ,Biol Psychiatry. 1;48(7):693-701[2000]). The present study had three main aims: To determine the effect of ECS on the behavior of FSL rats (an animal model of depression), to measure the levels of pregnenolone, DHEA and DHEA-S in plasma and brain after ECS, and to evaluate whether DHEA injected parallel to ECS treatment diminishes the behavioral effect of the ECS. Our results show that rats immobility in swim test, as a measure of depressive behavior, was reduced by ECS treatment $(49 \pm 5.53 \mathrm{sec} / 5 \mathrm{~min}$ vs $107 \pm 11.8 \mathrm{sec} / 5 \mathrm{~min}$ in controls, $\mathrm{p}<0.05)$. Injection of DHEA parallel to ECS treatment reversed the rats immobility almost to level found in na)ve-FSLs $(88 \pm 13.8 \mathrm{sec} / 5$ min). All groups that were injected with DHEA showed a significantly increase in serum and cortex levels of DHEA. This increase was dramatically high in the cortex of rats receiving ECS parallel to DHEA $(104.8 \pm 10.1 \mathrm{pmol} / \mathrm{gr}$ tissue vs $23.9 \pm 4.1 \mathrm{pmol} / \mathrm{gr}$ tissue in DHEA group, $\mathrm{p}<0.001)$, this group showed also a significant decrease in serum pregnenolone level compared to the three other groups. These results indicate that ECS in a rat model of depression alter serum and brain neurosteroids and this alteration may associate with behavioral outcome.

Keywords: Neurosteroids, DHEA, ECS, GABA.

The action of L-DOPA and other dopaminergic agents in hemiparkinsonian guinea pigs

Moses D. ' and Finberg J.P.M. ${ }^{2}$

${ }^{7}$ Neurology Dept., Rambam Medical Center, Haifa; ${ }^{2}$ Dept. of Pharmacology, Faculty of Medicine, Technion, Haifa

The rat made hemiparkinsonian by unilateral 6-hydroxydopamine injection into the nigro-striatal pathway is one of the most widely utilized Parkinson's disease models, however, rat striatum differs significantly from the human in MAO isoenzyme content and affinity to dopamine as well as in the pattern of dopamine metabolism. Guinea pig (GP) brain more closely resembles the human in the abovementioned parameters. We therefore studied turning behavior in the hemiparkinsonian GP. Male albino GPs were injected with 6-hydroxydopamine into the right substantia nigra. Rotational behavior was recorded after challenge with apomorphine, methamphetamine, and different doses of L-DOPA methylester. Animals were sacrificed after behavioral testing and dopamine content in striatal tissue was measured by HPLC with electrochemical detection. Apomorphine $(18 \mathrm{mg} / \mathrm{kg}$ ) and L-DOPA methylester $(4,8$ and $15 \mathrm{mg} / \mathrm{kg})$ induced turning contralateral to lesioned striatum; methamphetamine $(7 \mathrm{mg} / \mathrm{kg})$ induced ipsilateral turning. Apomorphine- induced turning was highly predictive of more than $95 \%$ striatal dopamine depletion. Apomorphine caused turning only at high dose but at lower doses it induced severe dyskinesias without turning. In animals with greater than $95 \%$ depletion of striatal dopamine, acute deprenyl treatment alone $(2 \mathrm{mg} / \mathrm{kg})$ induced ipsilateral turning but of lower intensity than direct agonists. GP showed greater sensitivity than rat to L-DOPA induced turning and may be a superior model than the rat to study the neuropharmacological aspects of L-DOPA and dopaminergic agonist treatment of Parkinson's disease.

Keywords: Parkinson's disease, Dopamine, Locomotor behavior

Real-time computing without stable states: a new framework for neural computation based on perturbations

Markram H. ${ }^{1}$, Maass W. ${ }_{1}^{2}$, Natschläger T. ${ }^{2}$, Melamed O. ${ }^{1}$, Rotem A. and Wasserstrom A.

${ }^{1}$ Dept. of Neurobiology, Weizmann Institute for Science, Rehovot, 76100, Israel; ${ }^{2}$ Institute for Theoretical Computer Science, Technische Universität Graz; A-8010 Graz, Austria

A key challenge for neural modeling is to explain how a continuous stream of multi-modal input from a rapidly changing environment can be processed by stereotypical recurrent circuits of integrate-and-fire neurons in real-time. We propose a new framework for neural computation that provides an alternative to previous approaches based on attractor neural networks. It is shown that the inherent transient dynamics of the highdimensional dynamical system formed by a neural circuit may serve as a universal source of information about past stimuli, from which readout neurons can extract particular aspects needed for diverse tasks in real-time. Stable internal states are not required for a stable output, since transient internal states can be transformed by readout neurons into stable target outputs due to the high dimensionality of the dynamical system. Our approach is based on a rigorous computational model, the liquid state machine that, unlike Turing machines, does not require sequential transitions between discrete internal states. Like the Turing machine paradigm, it allows for universal computational power under idealized conditions, but for real-time processing of timevarying input. The resulting new framework for neural computation has novel implications for the interpretation of neural coding, for the design of experiments and data-analysis in neurophysiology, and for neuromorphic engineering.

Keywords: Recurrent neural networks; Neural coding; Temporal integration

\section{Omega-3 fatty acid treatment of depressive breakthrough during unipolar maintenance} Nemets B. Stahl Z. and Belmaker RH. Ministry of Health Mental Health Center, Faculty of Health Sciences, Ben Gurion University of the Negev, Beersheva

Studies have reported that countries with high intake of fish oil have low rates of depression. We studied a specific omega- 3 fatty acid, the ethyl ester of eicosapentaenoic acid (E-EPA) as an adjunct to antidepressant treatment for breakthrough depression in recurrent unipolar patients on maintenance therapy. Design was four-week parallel group double-blind add-on to ongoing antidepressant therapy. Twenty patients participated, seventeen females and three males, all with diagnosis of current major depression.. Highly significant benefits were found by week 3 of treatment for eicosapentaenoic acid compared to placebo. It is not possible to distinguish whether eicosapentaenoic acid augments antidepressant action in the manner of lithium or has independent antidepressant properties of its own.

Keywords: Eicosapentaenoic acid, Omega-3, Fatty acid, Depression, Clinical trial 
Dextrorphan potentiates morphine analgesia without preventing morphine tolerance in preweanling rats Neufeld M. and Frenk H.

Dept. of Psychology, Tel-Aviv University, Ramat Aviv, Israel

Tolerance to both acute and chronic morphine administrations is a known problem, which hinders proper pain management in adult humans. NMDA antagonists are known to prevent the development of morphine tolerance and potentiate its acute analgesic effects in adult mammals. In infant animals the issue of morphine analgesia and tolerance is yet not fully understood. In the current research investigated the effects of the NMDA antagonist dextrorphan (dex) on the development and expression of morphine tolerance in rat pups. Two and eight days old rat pups were injected twice daily for a week with dex+morphine, saline + morphine, dex + saline or saline + saline. At 9 or 15 days of age, respectively, each group was tested with one of the above combinations and tested for analgesia using the tail flick paradigm. Dextrorphan was not able to prevent the development of morphine tolerance when chronically co-administered with morphine. It did, however, potentiate the analgesic effects of morphine even in morphine tolerant pups. Dextrorphan alone had a differential effect in both age groups, having an analgesic effect at age 15 but not 9 days. Furthermore, a non-analgesic dose at 15 days of age was not efficacious in potentiating morphine analgesia. These results may shed some light on the ontogeny of the interaction between NMDA and opiate receptors.

Keywords: NMDA, Tolerance, Infant rats

Protective and harmful autoimmunity in the CNS: experimental data and mathematical modeling Nevo U. ${ }^{1,2}$, Kipnis J. , Golding I. ${ }^{2}$, Akselrod S. ${ }^{2}$, Neumann A. ${ }^{3}$ and
Schwartz M.

IDepat. of Neurobiology, Weizmann Institute of Science, Rehovot; ${ }_{3}^{2}$ School of Physics and Astronomy, Tel-Aviv University, Tel-Aviv; ${ }^{3}$ Dept. of Biology, Bar-Ilan University, Ramat-Gan

Autoimmune response is widely viewed as an unfortunate outcome of the presence and activation of harmful immune components directed towards self-antigens. It is now evident, however, that autoimmune response, at least in the central nervous system (CNS), can be beneficial and 'protect'the tissue from damage. (Moalem et al., Nat. Med. 5:49-55 [1999]; Hauben et al., J.Neurosci. 20(17):6421-30 [2000]). This 'protective autoimmunity' is a spontaneous physiological response to insult, at least in some strains (Yoles et al., J. Neurosci. 21(11):3740-8 [2001]; Kipnis et al., J. Neurosci. 21:4564-4571 [2001]). Recently it became clear that the same $T$ cells are responsible for both the protective and the destructive (i.e., disease-related) activities associated with the autoimmune response, and that the way in which the response is regulated will determine whether its outcome is beneficial or harmful.

We have developed a mathematical model of the adaptive autoimmune response to CNS insult. Using basic rules for the dynamics of autoimmunity, with no discrimination between self and non-self, the following situations were simulated: (1) a wellregulated autoimmune response leading to inhibition of the degenerative process, thus reducing the damage; (2) a harmful autoimmune reaction resulting from an insult that occurs in the absence of a timely adaptive autoimmune response or in the presence of an inadequately regulated response; (3) a relapsing/remitting pattern of response to an insult, resulting from loose regulation coupled to intense immune activation. The model accommodates the novel view that protective autoimmunity can be conferred by any mechanism that allows an appropriately controlled adaptive anti-self response (Schwartz and Kipnis, TIMM 7(6):252-8 [2001]).

Keywords: Autoimmune, Protective autoimmunity, Immune model, Neuroprotection.
Comparison of multifocal electroretinogram topography between normal and ocular hypertensive Cynomolgus monkeys and humans Ofri R. ${ }_{\text {Raz D. }}$ Seeliger MW. ${ }^{2}$, Percicot CL. ${ }^{3}$, Lambrou GN. ${ }^{3,4}$ and

${ }^{1}$ Koret School of Veterinary Medicine, Hebrew University of Jerusalem, Israel; ${ }^{2}$ University Eye Hospital, Dept. II, Tuebingen, Germany; ${ }^{3}$ Novartis Ophthalmics, Basel, Switzerland; ${ }^{4}$ Dept. of Ophthalmology, Faculty of Medicine, Strasbourg, France

Purpose. To compare the functional retinal topography between cynomolgus monkeys and humans, and to look for differences in ocular hypertensive eyes. Methods. Multifocal electroretinograms (MF-ERGs) were recorded from both eyes of 9 9-year old Cynomolgus monkeys (Macaca fascicularis) with experimentally induced unilateral hypertension. After positioning, 7 minuterecordings were obtained, with a stimulus of 103 unscaled hexagons. First and second order responses were evaluated by single element analysis as previously described (Seeliger et al., Invest Ophthalmol Vis Sci 39:718-23 [1998]), and compared to human records. Results. The MF-ERG in monkey showed essentially the same amplitude and implicit time distribution found in humans, except for the lack of a marked delay in the region of the blind spot. In ocular hypertensive monkeys, amplitudes were generally larger, and the range of implicit time variations was greatly reduced both centrally and peripherally, together with an amplitude loss of the second order component. Conclusions. No substantial differences in the functional retinal topography between cynomolgus monkeys and humans were detected, reinforcing the value of this model. The widespread loss of implicit time variability in ocular hypertension may indicate global retinal damage and be related to the visual field loss. Supported by a grant from Novartis Ophthalmics (Ofri $R$ ) and by DFG grants SFB 430 C2 and Se837/1-1 (Seeliger MW) Keywords: Glaucoma, Multifocal electroretinogram, Animal model

In vivo evaluation of demyelination in mouse brain by magnetization transfer imaging

Olivson A. ${ }^{1}$, Ben-Hur T. ${ }^{2}$, Mizrachi-Kol R. ${ }^{2}$, Goelman G. ${ }^{1}$ ${ }^{T}$ Hadassah Hebrew University Hospital, Medical Biophysics Dept., MRI/NMR Laboratory, ${ }^{2}$ Hadassah Hebrew University Hospital, Neurology Dept.

Multiple sclerosis (MS) is a demyelinating autoimmune disease of the central nervous system. The relatively new magnetic resonance imaging techniques, exploiting the effect of magnetization transfer (MT) between immobile protons of macromolecules, such as myelin, and mobile protons of free water (MT-MRI), is accepted as a promising method in following the development of MS. This techniques is currently used to detect and quantify the white matter abnormalities in the brain and spinal cord of MS patients. However, the practical realization of MTMRI is not trivial and the results depend on the experimental set chosen. In addition, it is important to develop radiological means with experimental models of MS, since evaluation of efficacy of novel therapies currently relays solely on clinical and pathological grounds.

Our study is aimed to assess the ability of MT-MRI to follow in vivo the development of chronic experimental autoimmune encephalomyelitis (CR-EAE) in mice with further perspectives to use this techniques in validation of therapeutic treatments that cause remyelination. CR-EAE was induced by s.c. injection of $300 \mu \mathrm{g}$ MOG with complete freund adjuvant. The MT-MRI was performed on 4.7T Bruker Biospec system and the magnetization transfer ratio (MTR), was calculated for the control, untreated brain, and at different time points during CR-EAE development. The optimization of the experiment, methodology of data analysis and MTR changes induced upon demyelination, will be discussed. Keywords: Multiple sclerosis, Magnetization transfer MRI, Demyelination 
Astrocyte activation in vivo is modulated isoform-specifically by apolipoprotein E3 and not by apolipoprotein E4 Ophir G., Meilin S., Efrati M. and Michaelson D.M Dept. of Neurobiochemistry, Tel Aviv University, Tel Aviv Genetic and immunohistochemical studies revealed that the allele E4 of apolipoprotein $E$ (apoE) is an important risk factor for Alzheimer's disease (AD) and that the chronic brain inflammation associated with this disease is more pronounced in subjects who carry this allele.

In the present study, we employed mice transgenic for the human apoE isoforms apoE3 or apoE4 on a null mouse apoE background and intracerebroventricular injection of LPS, to investigate the possibility that brain inflammation is affected by the apoE genotype. Accordingly, LPS treatment of 12 months' old mice resulted in marked activation of brain astrocytes in the apoEdeficient and apoE4 transgenic mice but not in the control and apoE3 transgenic mice. LPS-dependent microglial activation, however, was not affected either by apoE deficiency or by the apoE3 and apoE4 transgenes. Furthermore, the brain apoE levels of the LPS-treated apoE4 and apoE3 transgenic mice were comparable. These results show that LPS-induced astrogliosis in apoE transgenic mice is regulated isoform-specifically by apoE3 and not by apoE4 and suggest a novel mechanism via which the phenotypic expression of the apoE genotype may be mediated. Keywords: Alzheimer's disease, Apolipoprotein E, Inflammation, Transgenic

\section{Effects of reversible inactivation of TE, perirhinal and}

entorhinal cortex on serial memory in monkeys.

Orlov T., Yakovlev V., Hochstein S. and Zohary E.

Dept. of Neurobiology, Hebrew University, Jerusalem 91904

Macaque monkeys can utilize a combination of different mnemonic strategies to retrieve lists of ordered images (Orlov et al., Nature 404:77-80 [2000]). These include ordinal-number categorization, stimulus-stimulus association and working memory. We found that categorization of the image ordinal number was the default and dominant mnemonic routine. We propose the existence of "category label" neurons (sensitive to images from a specific category), which encode the ordinal position of the images in long-term memory. The goal of the present experiment was to find cortical regions involved in image categorical memory. Two monkeys were trained with fractal images on a Serial recall task and a control DMS task. In one monkey, we reversibly inactivated sites in TE, perirhinal and entorhinal cortex by microinjection of the GABA-agonist muscimol while performing these tasks. Three mkl of $5 \%$ muscimol was injected bilaterally in 28 sites of perirhinal (10), enthorhinal (8), TE (6) and parahippocampal (4) areas. Localization of injection was controlled with MRI images. Reaction time (RT) in the serial recall task increased significantly after muscimol injections in 10 sites (TE -3 , perirhinal - 4, entorhinal $-5, \mathrm{P}<0.01)$. The mean $\mathrm{RT}$ increment relative to the baseline was $219.7+/-35.6 \mathrm{~ms}$ or $27.7+/-5.1 \%$ ). This was mirrored by an increase in the error rate $(10.6+\% 5.6 \%)$. No changes were observed following saline injections in the same sites. Muscimol injections in two sites in entorhinal cortex produced categorization specific changes in the RT. These results suggest that medial temporal cortical areas may be involved in ordinal number categorization and memory.

Keywords: Temporal order, Categorization, Muscimol, Perirhinal cortex, Entorhinal cortex.

\section{Can the Ca Voltage hypothesis account for calcium induced} release in the absence of depolarization?

Parnas H. and Jaimovich A.

The Otto Loewi Minerva center for cellular and molecular Neurobiology, Dept of Neurobiology, Alexander Silberman institute of life science, The Hebrew University, Jerusalem

The Ca-Voltage hypothesis suggests that the release of neurotransmitter from nerve terminals is governed by two factors. The first is the calcium concentrations in the vicinity of the release sites. The second is depolarization mediated relief of a tonic block imposed by presynaptic autoreceptors.

However. it was shown earlier (Schneggenburger and Neher, Nature 406:889-893 [2000]) that even in the absence of depolarization, elevation of intracellular $\mathrm{Ca}^{++}$to sufficiently high levels can promote release (hence denoted $\mathrm{Ca}$ induced release). These results seem to contradict the Ca-Voltage hypothesis.
We suggest a way to explain the above conflicting results in the framework of the $\mathrm{Ca}-$ Voltage hypothesis. We suggest that $\mathrm{Ca}$ induced release can be directly derived from the molecular scheme underlying depolarization induced release.

To check for this possibility, we constructed a kinetic model based on the molecular mechanisms that underlie the Ca-Voltage hypothesis, and the results of the model were compared to the experimental results of Schneggenburger and Neher.

The simulation results show that, as expected, even in the total absence of depolarization, the molecular basis underlying the $\mathrm{Ca}$ voltage hypothesis can produce substantial release. This occurs, providing that $\mathrm{Ca}$ concentration is significantly higher than under conditions where depolarization is applied. Furthermore, the hypothesis explains the different behavior of the time courses of $\mathrm{Ca}$ induced release and depolarization induced release.

Keywords: Ca-Voltage hypothesis, Ca-induced release autoreceptors.

\section{2-arachidonoyl glycerol (2-AG) alters neurotransmitters} following weight loss

Patursky I. , Avraham Y. ${ }^{1}$, Hao S. ${ }^{1}$, Ben-Shushan D. ', Mechoulam $\mathrm{R}^{2}$, Berry EM.

Depts. of Human Nutrition and Metabolism ${ }^{\prime}$ and the Dept of Medicinal Chemistry and Natural Products ${ }^{2}$, Hebrew UniversityHadassah Medical School, Jerusalem

Understanding the interaction between nutrition and brain function may lead to strategies for the treatment of the diseases associated with weight loss such including anorexia nervosa.

Cannabis sativa and its active constituent tetrahydrocannabinol (THC) are known to increase appetite. With the discovery of the endogenous cannabinoids - arachidonoyl ethanolamide (Anandamide, ANA), a fatty acid amide and 2-arachidonoyl glycerol (2-AG) a fatty acid ester - it became possible to examine the effect of these brain constituents on appetite.(Hao et al,
European J. of Pharmacology 392:147-156[2000]) 2-AG is present in both the brain and periphery and parallels THC and anandamide in most of their activities. $2-A G$ is also present in human and bovine milk. The activity of $2-\mathrm{AG}$ alone is low; but it can be significantly increased by 2-palmitoylglycerol and 2 linoleoylglycerol which are present in the body alongside 2-AG. They enhance its activity by delaying hydrolysis and lowering the uptake rate into the cells - the so-called "entourage" effect.

We have shown that, in young female Sabra mice, very low doses of $2 \mathrm{AG}+$ entourage $(0.001 \mathrm{mg} / \mathrm{kg})$ affect food consumption, cognitive function, cathecholaminergic and serotonergic pathways. NE, dopamine, 5-HT and their intermediates increased significantly in the hypothalamus While in the hippocampus dopamine, Dopac and 5HT increased significantly and these effects were reversed after treatment with a CBI receptor antagonist.

The use of small quantities of endocannabinoids to avoid psychotropic side effects might have important implications for the possible treatment of anorexia nervosa

Keywords: 2-AG, Neurotransmitters, Weight loss, CB1 receptor antagonist.

\section{Differentiating the types of attention required for feature and} conjunction search

Pavlovskaya M. ${ }^{1}$, Ring H. ', Groswasser Z. ${ }^{1}$ and Hochstein S. ${ }^{2}$ 'Loewenstein Rehabilitation Hospital, Raanana, Sackler School of Medicine, Tel Aviv University and ${ }^{2}$ Hebrew University, Jerusalem, Israel

We address a long-standing conflict in the visual search literature by studying search performance of neglect patients using laterally presented search arrays, and comparing performance on feature and conjunction search tasks. The issue relates to whether feature search is performed "pre-attentively" i.e. supposedly independently of attention(Treisman \& Gelade, Cogn. Psychol., 12, 97-136 [1980]) or with "spread attention" i.e. irrespective of the number or eccentricity of the elements, but demanding attention, nevertheless (Treisman, $Q$. J. Expl Psychol. A, 40, 201237 [1988]).These questions address the very nature of attention and of feature search. If neglect had to do only with focusing attention, and if feature search were a pure pre-attentive task, we would expect that left-neglect patients would have no problem in performing a feature task in the left visual field.

Five neglect patients and six normal controls were tested with stimulus arrays composed of $3 \times 3,5 \times 5$ or $7 \times 7$ elements presented centrally, or in the left (LHF) or right hemifield (RHF) at three 
possible eccentricities. Array centers were at $0^{\circ}$ or $2.5-6.5^{\circ}$ of visual angle from the fixation cross. We found conclusive evidence that feature search is definitely susceptible to inattention effects. When search arrays are placed centrally, neglect subjects have a more difficult time finding targets that fall in the neglected hemi-array. When arrays are placed laterally, there is a tremendous difference between neglect subject performance in finding "pop-out" targets when the array is in their neglected hemifield than when it is in the attended hemifield.

We conclude that the difference between feature and conjunction search is indeed related to attention however, not to the need for or independence of attention, but rather in the type of attention needed. Feature search requires spread attention, as indicated by its parallel processing mode, while conjunction search requires focused attention, giving it a serial nature and set-size dependence.

Keywords: Visual attention, Feature search, Conjunction task

\section{Neural correlates during learning of new visuomotor mapping} Paz R., ${ }^{1,2}$, Boraud T. ${ }^{1,3}$, Natan C. ${ }^{1}$, Bergman $\mathrm{H}^{1,2}$ and Vaadia ${ }_{1}^{1,2}$ Israel; ${ }^{2}$ The Interdisciplinary center for neural computation, Hebrew University, Jerusalem. Israel; ${ }^{3}$ Laboratoire de Neurophysiologie, CNRS UMR 5543, Universite Victor Segalen, Bordeaux, France

We examined activity of neurons in primary motor cortex (MI) while a monkey was learning a new visuomotor mapping.

The task includes two epochs. The first included standard 8 directions center-out trials ("standard epoch"). In the second, we introduced unfamiliar transformations (rotation) between the manipulanda and the cursors motion on the screen. At the end these two epochs ( $\sim 350$ trials), an additional set of similar two epochs (with the same transformations) was repeated. Each day ("session") a different transformation was introduced.

We compared neuronal activity between the matching standard epochs in the two sets, both in the preparatory activity (a hold period) and in the movement related activity.

We found that the activity of many of the task related neurons was significantly modified, especially in properties of their tuning curves. Some exhibited change in preferred direction, some in baseline activity and some in amplitude.

Furthermore, we used the population vector method to predict the actual movement from the neuronal activity and found that the accuracy of prediction improved in the second set of standard movement (after the transformation epoch) compared to the first set. This improvement was specific to the direction of the transformation done in that session and only when performed with neurons from that session.

We conclude that changes of neuronal activity in MI occur during learning of new visuomotor mapping and suggest that they reflect reorganization of neuronal networks that supply better information on the newly acquired behavior.

Keywords: M1, Motor cortex, Electrophysiology

\section{Goi-GDP negatively regulates the basal activity of $G$ protein} activated $\mathrm{K}^{+}(\mathrm{Kir} 3)$ channe

Peleg S., Ivanina T. and Dascal N.

Sackler School of Medicine, Tel Aviv University, Tel Aviv 69978

The GIRK channels display basal activity $\left(\mathrm{I}_{\text {basal }}\right)$; acetylcholine $(\mathrm{ACh})$ activates GIRK via $\mathrm{m} 2$ receptor $(\mathrm{m} 2 \mathrm{R})$, causing an evoked current $\left(\mathrm{I}_{\mathrm{ACh}}\right)$. The activation is mediated by direct binding of $\mathrm{G}_{\beta \gamma}$ released from $\mathrm{G}_{\mathrm{i} / \mathrm{o}}$ proteins, to GIRK. Recently, we have proposed that $\mathrm{G} \alpha$ may play an important role in GIRK channel gating. This was after we have found, unexpectedly, that increasing the density of channels leaded to a correlated increase in $\mathrm{I}_{\text {basal }}$, whereas the extent of activation by $A C h, R_{a}$ (ratio $I_{A C h} / I_{\text {basal }}$ ) was reduced. We assumed that there is a blocking molecule, present in Xenopus oocytes in limited amounts, that keeps GIRK closed at rest. Coexpression of $G \alpha_{i 3}$ or $G \alpha_{i 1}$ restored low $I_{\text {basal }}$ and increased $R_{a}$. We next explored the question which form of $G \alpha_{i}$ inhibits the channel at rest. Is it $\mathrm{G} \alpha_{\mathrm{GTP}}, \mathrm{G} \alpha_{\mathrm{GDP}}$ or the heterotrimer $\mathrm{G} \alpha \beta \gamma$ ? We prepared $\mathrm{G} \alpha$ mutants: $\mathrm{G} \alpha_{13} \mathrm{Q} 205 \mathrm{~L}$ (constitutively active, GTPbound), $\mathrm{G} \alpha_{\mathrm{i} 3} \mathrm{G} 204 \mathrm{~A}$ (constitutively inactive, GDP- bound) and coexpressed them with the channel at high density. Constitutively inactive $G \alpha_{13}$ reduced $I_{\text {basal }}$, whereas the constitutively active $G \alpha_{13}$ had much smaller and variable effects. We have done also experiments in excised patches with purified proteins $G \alpha_{i l G D P}$ alone and with $G_{\beta \gamma}$ subunit mixed earlier to form heterotrimer.
Preliminary experiments suggest that purified $\mathrm{G} \alpha_{\mathrm{GDP}}$ inhibited the channel most efficiently. In addition biochemical experiment showed better binding of $G \alpha_{\mathrm{GDDP}}$ than the heterotrimer $\left(G \alpha_{1} \beta \gamma\right)$ to the channel. We propose that the active regulator of the basal activity of GIRK is $\mathrm{G \alpha}_{\mathrm{iGDP}}$ alone, however the heterotrimer remains a plausible candidate.

Keywords: GIRK channel, G protein, Gating

Reduce sensorimotor cortical basal activity in a rat model of Parkinson's disease as measured by fMRI

Pelled G. ${ }^{1}$, Bergman $\mathrm{H}^{2}$ and Goelman $\mathrm{G}$

HBRC, medical biophysics and nuclear medicine dept., Hadassah Hebrew University Hospital, Jerusalem, Israel. physiology dept. Hadassah Medical School, The Hebrew

University, Jerusalem, Israel

The unilateral 6-OHDA rat model of Parkinson's disease (PD) is a well established model for studying the functional changes that occur in the brain as a consequence of the dopaminergic lesion. Our previous findings have shown significantly bilateral sensorimotor cortical activity, compare to sham-operated rats, as measured by functional magnetic resonance imaging (fMRI) Since fMRI measures the changes in the oxy/dioxy-hemoglobin, we examined if this higher fMRI signal is a result of over activity or if it corresponds to changes in the cortical basal level. For this purpose we measured the sensorimotor basal level in $6 \mathrm{PD}$ model rats and 6 sham-operated rats using fMRI. 70 Gradient-Echo EPI images of 4 cortical slices were obtained during rest. Regions of interest (ROI) of the right and the left motor and sensory cortex were chosen and their intensity was normalized. The analysis was done in two different ways: 1). Calculation of the mean and the standard deviation (SD) of all the pixels in the ROI of each image and then their average along time. 2). Calculation of the mean and SD of each pixel in the ROI along time, and then the average of all the pixels. Our results show that there is a significant decrease $(\mathrm{P}<0.01)$ of the mean basal activity level in the motor and sensory cortex of the PD model rats compare to the sham-operated rats. These results agree with the classical basal ganglia circuit model, and explains the discrepancy of our previous results with the model.

Keywords: fMRI, BOLD, Parkinson's disease, Sensorimotor cortex

Observation of high correlation between fMRI signal and dendrites density

Pelled G. ... Bergman $\mathrm{H}^{2}{ }^{2}$ and Goelman G. ${ }^{1}$

HBRC, medical biophysics and nuclear medicine dept.

Hadassah Hebrew University Hospital, Jerusalem, Israel. ${ }^{2}$ physiology dept., Hadassah Medical School, The Hebrew University, Jerusalem, Israel

Measuring the functional magnetic resonance imaging bloodoxygenation-level-dependent (BOLD) signal simultaneously with electrophysiology recording, has shown high correlation of the BOLD signal with the local field potentials (LFP) in the visual cortex of the monkey (Logothetis et al, Nature 412:150-157 [2001]). The LFP recording represents the summation of the activity in the dendrites, as opposed to single unit recording, which represents the output activity of the neuron. Measuring the BOLD signal in the different layers of the cortex, can expand our understanding of the neuronal components it relates to, since the cortex is composed of 6 layers when the lower layers consist mostly of the cell bodies and the dendrites, while the upper layers consist the white matter. The BOLD technique measures the changes in the oxy/dioxy-hemoglobin ratio during rest represents the basal level activity. We measured the BOLD signal in six urethane-anesthetized rats during rest. Four slices in the cortex were chosen, and 50 Gradient-Echo EPI images were obtained. Regions of interest (ROI) in the motor and sensory cortex were chosen and their intensity was normalized. The analysis was done in two different ways: 1). Calculation of the mean and the standard deviation (SD) of all the pixels in the ROI of each image and then their average along time. 2). Calculation of the mean and SD of each pixel in the ROI along time, and then the average of all the pixels. Our results show that the mean of the ROI intensity is similar in both analysis, but the SD is different, and that the lower layer of the cortex display significantly $(\mathrm{P}<0.01)$ the largest BOLD signal. The latter result supports Logothetis results, that the BOLD technique is correlated to the dendritic component of the neuronal activity.

Keywords: AMRI, BOLD, LFP 
Functional correlation maps of inter-laminar pyramidal neurons

PennY., Silberberg G., Wu C.Z. and Markram H.

Dept. of Neurobiology, Weizmann Institute of Science, Rehovot

The neocortex is parcellated into numerous functional columns that are approximately the diameters of the basal dendritic and axonal arbors of pyramidal neurons and stretch across all six layers. The intriguing feature of functional columns is that there are no anatomical boundaries at the microcircuit level, suggesting that boundaries are formed dynamically depending on the topography and structure of the input. In order to explore the evolution and dynamics of the activity in microcircuits we obtained multineuron whole-cell recordings of pyramidal neurons across layers and across columns. Sub-threshold cross correlations were obtained before, during and after the network was stimulated with a solution containing high $\left[\mathrm{K}^{+}\right]$and low divalent ions. Preliminary data indicates specific interlayer and cross layer correlation maps.

Keywords: Pyramidal cells; Neocortex; Interlayer; Intercolumnar;

Subthreshold cross-correlations.

Interaction of KCNE1 and KCNQ1 C-termini: implication for $I_{K S}$ channel assembly and gating

Peretz A. ${ }^{\text {, Schottelndreier H. }}$, Schmitt N. ${ }^{2}$, Gibor G. ${ }^{1}$, Pongs O. ${ }^{2}$ and Attali B.

Dept. of Physiology and Pharmacology, Sackler School of Medicine, Tel Aviv University, Israel Institut fuer Neurale Signalverarbeitung, ZMNH, Hamburg, Germany.

The I $\mathrm{ISS}_{\text {s }}$ potassium channel complex consists of the heteromultimeric assembly of two structurally distinct $\alpha$ and $\beta$ subunits called KCNQ1 and KCNE1, respectively. Mutations in KCNQ1 and KCNE1 genes produce the long QT (LQT) syndrome, a human genetic cardiovascular disease. We recently identified a small domain, cad, in the KCNQ1 C-terminus (aa. 589-620) which functions as an assembly domain :or KCNQ1 $\alpha$ subunits. KCNQ1 do not express functional $\mathrm{K}^{+}$channels in transfected-CHO cells without this domain. Co-expression of KCNQ1 510-620 with WT KCNQ1 suppresses $\mathrm{K}^{+}$channel activity, probably by inhibiting the tetrameric assembly of WT subunits in a dominant-negative fashion. A Jervell and LangeNielsen (JLN) mutation eliminates this assembly domain, suggesting that JLN patients are defective in KCNQ1 subunit assembly. Co-expression of KCNE1 with KCNQ1 510-620 and WT KCNQ1, fully restores a $\mathrm{I}_{\mathrm{KS}}$-like $\mathrm{K}^{+}$channel activity. Conversely, a naturally occurring C-terminal mutant of KCNE 1 (KCNE1W87R) is unable to restore functional $\mathrm{K}^{+}$currents, as does WT KCNE1. It is noteworthy that the KCNE1 W87R mutant exhibits altered gating properties, relative to WT KCNE1, with rightward shift of the voltage-dependence of activation and faster deactivation kinetics. In contrast, a N-terminal deletion mutant of KCNE1 (KCNE1 $\triangle 39-43$ ) is capable to retrieve functional $\mathrm{K}^{+}$ channel activity, when co-expressed with KCNQ1 510-620 and WT KCNQ1. In all, these data suggest that the KCNE1 $\beta$ subunit protects WT KCNQ1 $\alpha$ subunits from the dominant-negative inhibition of the tetrameric assembly exerted by KCNQ1 510-620. This protecting effect is exerted through an interaction of the KCNE1 C-terminus with the cad of KCNQ1

Keywords: Potassium channels, LQT, KCNQ, KCNE

PI3 kinase activation underlies GDNF-mediated protection against 6-OHDA in dopaminergic cells

Perez R., Ding Y-M., Jaumotte J., Klann E. ${ }^{2}$, Lin E., Ugarte S., and Zigmond $M$.

University of Pittsburgh School of Medicine; ${ }^{2}$ Baylor College of Medicine, USA

Parkinson's disease (PD) is associated with selective loss of dopamine (DA) neurons in the substantia nigra (SN). GDNF, a potent neurotrophic factor, is protective in animal models of PD and we have explored the mechanisms underlying this neuroprotective action in several in vitro systems. First, primary cultures were prepared from the SN-rich region of neonatal rat mesecephalon. Antibodies to tyrosine hydroxylase (TH) and to GABA were used to visualize these two types of neurons. Cultures were treated for 15 min with 6-hydroxydopamine (6-OHDA, $<100$ (m), a neurotoxin that is concentrated in DA neurons and can initiate oxidative stress. 6-OHDA selectively destroyed $\mathrm{TH}+$ cells with little or no damage to GABA neurons. Pretreatment with the DA transport inhibitors nomifensine (10 (M) or GBR12909 (2 (M) prevented loss of DA neurons, providing further evidence of specificity. Much of the death caused by 6-OHDA appeared apoptotic, and this was blocked by the caspase 3 inhibitor $z$ DEVD-fmk. GDNF $(100 \mathrm{ng} / \mathrm{ml})$ prevented the development of TUNEL staining, suggesting that it, too, was able to block apoptosis. To explore the signaling cascades underlying the action of GDNF we used a dopaminergic cell line, MN9D. GDNF treatment activated both Erk1/2 and Akt as measured on western blots. Although the MEK inhibitor UO126 $(20(\mathrm{~m})$ did not reduce the neuroprotective effects of GDNF, the effect was blocked by the PI3 kinase inhibitor LY294002 (50 (m). Thus, our data suggest that the PI3K/Akt pathway is an important mediator of GDNF's ability to inhibit the oxidative stress-induced apoptosis in DA neurons.

Keywords: Cell death, Oxidative stress, Parkinson's disease, Trophic factors

\section{$\alpha 7$ acetylcholine receptor mRNA in peripheral blood} lymphocytes: A possible marker for schizophrenia Perl O. ${ }^{\text {, Ilani T. }}{ }^{1}$, Strous R.D. ${ }^{2}$, Lapidus R. ${ }^{2}$, and Fuchs S. ${ }^{1}$ ${ }^{1}$ Dept. of Immunology, Weizmann Institute of Science, Rehovot 76100, Israel; ${ }^{2}$ Beer Yaacov Mental Health Center

Acetylcholine mediates synaptic transmission in the vertebrate central nervous system through the activation of two major receptor subtypes, the muscarinic and nicotinic acetylcholine receptors (AChRs). Several recent studies have suggested that nicotinic $\alpha 7$ AChR may be associated with some aspects of schizophrenia. In search for peripheral biological markers for schizophrenia that may enable measurable and rapid diagnosis of this disorder we have investigated $\alpha 7 \mathrm{mRNA}$ levels in peripheral blood lymphocytes (PBLs) of schizophrenic patients and healthy controls. Blood samples were collected from 34 medicated and unmedicated (drug naïve) schizophrenic patients, and from 21 healthy smokers and nonsmokers. RNA was prepared from isolated lymphocytes and its amount and quality determined. PCR products specific for human $\alpha 7 \mathrm{AChR}$ were quantified by densitometry using Scion image analysis software. A significant decrease (20-95\%) of $\alpha 7 \mathrm{mRNA}$ levels in PBLs of schizophrenic patients has been observed, compared with controls. The decrease in $\alpha 7$ mRNA levels was not a result of medication, since unmedicated patients showed the same range of reduction as hospitalized schizophrenic patients. In addition, we have ruled out the possibility that the observed decrease in $\alpha 7$ mRNA levels resulted from nicotine consumption by smoking, as healthy smokers exhibited the same levels of $\alpha 7$ mRNA as nonsmokers. If the observed changes in $\alpha 7 \mathrm{mRNA}$ levels in PBLs, reflect the state of this receptor in the brain, our findings support the assumption that a deficit in $\alpha 7 \mathrm{AChR}$ is involved in the pathophysiology of schizophrenia. We propose that $\alpha 7$ AChR mRNA may serve as a peripheral marker for schizophrenia.

Keywords: Peripheral blood lymphocytes (PBL); $\alpha 7$ acetylcholine receptor; Schizophrenia; mRNA; RT-PCR.

Activity-dependent neuroprotective protein (ADNP) gene expression is developmentally regulated

Pinhasov A. ${ }^{1}$, Goldsweig AM. ${ }^{\text {, }}$, Brenneman D.E. ${ }^{2}$, and Gozes I. ${ }^{1}$ 'Dept. of Clinical Biochemistry, Sackler School of Medicine, Tel Aviv University, Tel Aviv, Israel 69978; ${ }^{2}$ SDMP, Laboratory of Developmental Neurobiology, NICHD, National Institute of Health, Bethesda, MD 20892 USA.

The glial-derived Activity-Dependent Neuroprotective Protein (ADNP) is associated with cellular protection (J. Biol. Chem. 276: 708-714 [2001]). ADNP is widely distributed with increased expression in the hippocampus, cerebral cortex and cerebellum. ADNP contains an 8 amino acid peptide NAPVSIPQ (NAP) that exhibits neuroprotective activity, stimulates brain development $(J$. Neurochem.72:1283-1293 [1999]) and improves learning and memory (J. Pharmacol. Pharm. Exp. Therap. 293:1091-1098 [2000]). To further evaluate the role of ADNP in the organism and its importance in the CNS, the expression of the protein during development was evaluated. Using whole mount in situ hybridization, ADNP mRNA was detected at early stages of the mouse embryonic development (E9.5). RNA was extracted daily from embryonic mouse brains (E12-birth). Utilizing Northern blot hybridization, ADNP mRNA was detected as constitutively expressed at all stages tested, with an apparent slight decline at birth. In comparison, when RNA was extracted daily from whole embryos (E7-birth), a peak ADNP expression was observed at E9.5 followed by a gradual decline toward birth 
Postnatal expression of ADNP mRNA was measured in mouse brains at day 1 , week 3 , month 3 and month 18 . Results showed that ADNP gene expression decreased during maturation up to postnatal day 21 , then reached a plateau and declined with aging. Taken together, these data indicate an important role for ADNP during brain development and aging.

Support: ISF, BSF, Neufeld, ISOA, Gildor Chair.

Keywords: ADNP, Northern blots, In situ hybridization, Gene expression.

\section{Down-regulation of central nervous system focal inflammatory responses}

Pinto F., Lazarovici P. and Brenner T.

Laboratory of Neuroimmunology, Dept. of Neurology, HadassahHebrew University Medical Center, Jerusalem

Inflammation of the central nervous system (CNS) occurs during various neurological disorders such as infections, multiple sclerosis and following injury. It involves activation of glial cells, which both respond to, and synthesize a range of inflammatory mediators (cytokines, free radicals and lipid mediators) within the CNS.

Stimulated glial cells are a commonly used model for CNS inflammation. However, individual cell type responses are influenced by the presence of other cell types due to contact or interaction, secretion of modulatory factors, etc.

Thus, the attempt to understand the sequence of events leading to the irreversible CNS damage caused by the inflammatory process, is hampered by the questionable relevance of the various in vitro models to the actual in vivo situation. Therefore, in the present study we used glial-neurons co-cultures in an attempt to elucidate the roles of neurons in the production of inflammatory mediators by LPS-stimulated glial cells.

Pervious studies in our laboratory indicated that rat stimulated glial cells induce the expression and the secretion of phospholipase $\mathrm{A}_{2}$ and that its stimulation is associated with increased production of the inflammatory mediators $\mathrm{PGE}_{2}$ and NO.

Culturing neurons with mixed glial cells caused a marked decrease in the production of nitric oxide and prostaglandins. In addition we found a reduction of PLA2 activity as compared with cultures containing only glia.

The finding that the presence of neurons in the culture reduces the secretion of inflammatory mediators supports our initial hypothesis that a combination of different cell types provides a more accurate research system.

Keywords: Central nervous system

Mechanisms of EAE-associated sickness behavior

PollakY. ${ }_{2}^{1}$ Ovadia $\mathrm{H}^{2}{ }^{2}$, Orion $\mathrm{E} .^{1}$ and Yirmiya $\mathrm{R}^{1}{ }^{1}$

'Dept. of Psychology, The Hebrew University of Jerusalem, Mount Scopus, Jerusalem, Israel; ${ }^{2}$ Dept. of Neurology, Hadassah-

Hebrew University Hospital, Jerusalem, Israel

Experimental autoimmune encephalomyelitis (EAE) is an inducible disease in which Th1 response activates effector cells and elicits inflammation and tissue damage within the central nervous system. Recently we showed that a behavioral syndrome precedes and accompanies the acute phase of the disease, as well as subsequent relapses. The behavioral syndrome consists of decreases in food intake, sucrose solution consumption and social exploration. To explore the mechanisms underlying the behavioral alterations, competitive RT-PCR and radio immunoassay (RIA) studies were conducted. These studies showed elevations in the expression of pro-inflammatory cytokines, such as interleukin (IL)- 1 and 6 and tumor necrosis factor $\alpha$ (TNF- $\alpha$ ), and prostaglandin $\mathrm{E}_{2}\left(\mathrm{PGE}_{2}\right)$, concordant in time with the behavioral alterations. Further studies showed that administration of IL-1 receptor antagonist (IL-1ra) and indomethacin, a prostaglandins synthesis inhibitor, attenuated some of the components of the behavioral syndrome. In contrast, pentoxifylline, a TNF- $\alpha$ inhibitor, had no affect on the behavioral symptoms. Taken together, our data suggest that inflammatory mediators underlie several components of the behavioral alterations associated with EAE.

Keywords: EAE, Sickness behavior, Pro-inflammatory cytokines, Prostaglandins
An EEG Study of Neural Integration in Schizophrenia During a Visual Somatosensory Illusion Pressman A. ${ }^{1}$, Peled A. ${ }^{2}$, Modai I. ${ }^{2,3}$ and Geva A.B. 'Dept. of Electrical and Computer Engineering Ben-Gurion University of the Negev, Beer Sheva, Isracl; ${ }^{2}$ Institute for Psychiatric Studies, Sha'ar Menashe Mental Health Center, Mobile Post Hefer 38814, Hadera, Israel; ${ }^{3}$ Bruce Rappaport Faculty of Medicine, Technion, Israel Institute of Technology, Haifa

The neuronal mechanisms that provide for a rapid interaction between visual and somatosensory neuronal systems probably involve short-term synaptic plasticity and neuronal reorganization. In this study we investigate neuronal processing and organization of the rubber hand illusion (RHI) with EEG evoked responses. The RHI is an illusion in which tactile sensations are referred to synthetic alien limb, it was first described by Botvinick and Cohen (1998), which have proposed that this illusion speaks of a threeway interaction (i.e., integration) among vision, touch and proprioception.

Going from pre-illusion condition to an illusion state a marked dipole (i.e., depolarization of electrical recording) over the central (parietal) and occipital regions is evident in control subjects. This dipole is evident up to around 0.2-millisecond range i.e., early processing intervals that probably reflect information processing of lower-level neuronal hierarchical systems such as those of unimodal multimodal association regions.

In schizophrenic patients these relations of hierarchical organization during the formation of the RHI are significantly altered. Early wave-forms of the evoked responses do not differ much at the time of the illusion compared non illusion epochs. The conditions associated with the illusion prevails only after 0.4 milliseconds. From this work it seems that the hierarchical organization of information processing in schizophrenic patients is disturbed compared to controls.

\section{Calcium dynamics in secretory vesicles}

Raveh A., Blank P., Coorssen J., Epstein J., Shani L., Zimmerberg J. and Rahamimoff $R$.

Dept. of Physiology and the Bernard Katz Minerva Centre for Cell Biophysics, Hebrew University Hadassah Medical School, POBox 12272, Jerusalem 91120, Israel; The Laboratory for Cellular and Molecular Biophysics, NICHD, NIH, Bethesda, MD, USA.

Membrane fusion is a fundamental cellular process involved in transmitter release and fertilization, triggered by elevated $\mathrm{Ca}^{2+}$ concentrations $\left[\mathrm{Ca}^{2+}\right]$. Extracellular and intracellular sources may contribute to this elevation. Since secretory vesicles contain high $\left[\mathrm{Ca}^{2+}\right]$, we examined its dynamics in sea-urchin eggs, using confocal microscopy and stochastic analysis of the fluorescence data. The study of the fluctuations in fluorescence intensities in intact eggs, shear-isolated membrane cortices and vesicles, labeled with fluorescent $\mathrm{Ca}^{2+}$ indicators (Fluo-4 and Fluo-4 AM), revealed a complex calcium dynamics..

The following observations were made:

1. The fluorescence intensity decayed faster in secretory vesicles than fluorescent beads or cytoplasm, most probably due to bleaching in a confined volume

2. The variance of the fluorescence intensity was higher than that of beads of comparable mean intensity.

3. Calcium waves were inferred from oscillations in the intravesicular fluorescence signal.

The non-stationary behaviour of the calcium signals in the secretory vesicles may indicate that the vesicles have a role in regulation of the fusion process. It may also suggest the existence of a novel pathway of presynaptic regulation of transmitter release.

Supported by the US-Israel BSF and ISF

Keywords: Calcium, Secretory vesicles, Fluctuation analysis

Isolated housing in laboratory rats: selective or non selective effects on drug seeking behavior

Raz S. and Berger B.D.

Dept. of Psychology, University of Haifa, Haifa

Isolated housing increases aggression and interferes with the performance of a cooperation task in male rats. Although the behavioral mechanisms for these actions of isolated housing are not known, presumably isolated housing increases or exacerbates irritability or stress that are also known to influence drug seeking behavior in humans. 
The present series of studies attempts to determine if isolated housing affects voluntary morphine consumption in laboratory rats and if so, to further determine the selectivity of this effect. Adult male Wistar rats, were housed in one of two conditions: isolated housing (1 per cage) or social housing (2 per cage). Intake of water alone, water + morphine $\mathrm{HCl}(0.5 \mathrm{mg} / \mathrm{ml})$, water + quinine $\mathrm{HCl}(0.024 \mathrm{mg} / \mathrm{ml})$ or water $+\mathrm{d}$-amphetamine sulfate $(0.025 \mathrm{mg} / \mathrm{ml})$ were measured repeatedly over several weeks both in one - bottle (forced) and in two - bottle (choice) tests.

Isolated housing significantly increased intake of morphine, quinine, and amphetamine across the series of forced - choice trials. However when morphine, quinine, or amphetamine were presented Vs water in a series of choice trials, isolated housing significantly increased intake only of morphine and quinine. There were no differences in water intake between the groups in these choice trials.

These results indicate that environmental and situational factors influence drug intake in laboratory rats as they do in humans, and thus support the utility of this animal model to the study of drug seeking behavior in humans. More studies are required in order to evaluate the conditions under which drug intake is affected by these manipulation.

Keywords: Drug seeking behavior, Isolated housing, Morphine.

Dissociation of working and reference memory in LTPdeficient GluR1-/- mice

Reisel D. ${ }^{1}$, Schmitt W. ${ }^{1}$, Andersen P. ${ }^{3}$, Seeburg P.H. ${ }^{2}$, Sakmann $\bar{B}^{2}{ }^{2}$, Sprengel R. ${ }^{2}$, Deacon R.M.J. ${ }^{1}$, Bannerman D.M. ${ }^{P}$ and Rawlins J.N.P.

Dept. of Experimental Psychology, University of Oxford, UK; ${ }^{2}$ Max-Planck Institute for Medical Research, University of Heidelberg, Germany; ${ }^{3}$ Dept. of Physiology, University of Oslo, Norway

L- $\forall$-amino-3-hydroxy-5-methylisoxazole-4-propionate (AMPA) receptor activity is required for long-term potentiation (LTP), a postulated model of cellular mechanisms underlying learning and memory. GluR1 (GluR-A), one of four AMPA receptor subtypes, is thought to mediate a postsynaptic mechanism of LTP expression. Previous studies have demonstrated that GluR1deficient mice lack LTP in the afferent pathways to the CA1 area of the hippocampus, yet their spatial reference memory is intact (Zamanillo et al, Science 284:1805-1811 [1999]). The present study investigated the role of AMPA GluR1 in working memory. Using male GluR $1^{-}$mice, we examined the functional significance of this receptor subtype in a rewarded alternation task on the elevated T-maze, a measure of spatial working memory. Performance on the T-maze was dramatically impaired by GluR 1 deletion (wild-types: $81.0 \%$, mutants: $50.8 \% \mathrm{~F}(1,26)=58.4$; $\mathrm{p}<0.0001)$. We then tested the same GluR $1^{-1}$ mice on the standard version of the Morris water maze task, a measure of spatial reference memory. In agreement with the previous study, performance in the Morris water maze was unimpaired; there was no difference between GluR $1^{--}$mice and controls on a probe test with the hidden platform removed (wild-types: $44.6 \%$, mutants: $46.9 \% \mathrm{~F}<1 ; \mathrm{p}>0.2)$. These results support an important role for AMPA GluR1 in spatial working memory but not in spatial reference memory.

Keywords: AMPA, GluR1 (GluR-A), Working memory.

$\mathrm{Na}^{+}$ions may be an important second messengers acting via

GBy

Rishal I. and Dascal N.

Dept. of Physiology and Pharmacology, Sackler School of Medicin, Tel Aviv University, Tel Aviv69978

The G-protein activated inwardly $\mathrm{K}^{+}$channels (GIRK) mediate postsynaptic inhibitory effect of various transmitters in brain and in atrium via 7-helix, G-protein coupled regeptors (GPCRs). GIRKs are normally activated by neurotransmitters via direct binding of $G \beta \gamma$ subunits released from $G \alpha_{i} \beta \gamma$ heterotrimers. However, GIRKs are also activated by cytosolic $\mathrm{Na}^{+}$. An aspartate in the proximal C-terminus of GIRK2 (D226N) is crucial for the gating by $\mathrm{Na}^{+}$(Ho et al, J. Biol. Chem. 274: 8539-8648 [1999]). Surprisingly, channel composed of GIRK $1_{\mathrm{WT}}\left(\mathrm{Na}^{+}\right.$-insensitive subunit) and GIRK2 2 226N (a single point mutation, that abolishes the direct $\mathrm{Na}^{+}$-dependent activation of GIRK) behaves in a same way as wild-type channel. $\mathrm{Na}^{+}$influx increases $\mathrm{I}_{\text {basal }}$ of GIRK1/2 and GIRK1/GIRK2 2226 similarly, an effect that is strongly affected by c $\beta A R K$ and is fully eliminated when $\mathrm{I}_{\mathrm{N}}$-train is given in the presence of tetrodotoxin (TTX). In excised Xenopus oocyte membrane patches, elevated $\mathrm{Na}^{+}$activates a GIRK $1_{\mathrm{F} 1375}$ channel (which, unlike the GIRK $1_{\mathrm{WT}}$, is able to form functional homotetrameric channels devoided of a direct $\mathrm{Na}^{+}$effect) and this effect is G $\beta \gamma$-dependent. To explain the above results, we proposed that $\mathrm{Na}$ causes the release of $\mathrm{G} \beta \gamma$ from $\mathrm{G} \beta \gamma$-binding proteins and, thus, elevates free G $\beta \gamma$ concentration. Direct binding experiments showed that $\mathrm{Na}^{+}$reduces the affinity of $\mathrm{G} \beta \gamma-\mathrm{G} \alpha_{\mathrm{GDP}}$ binding, supporting our hypothesis. Thus, an important conclusion from the work done so far is that there are two processes of GIRK activation by $\mathrm{Na}^{+}$, one direct and other $\mathrm{G} \beta \gamma$-dependent and that $\mathrm{Na}^{+}$may be an important second messenger acting via $\mathrm{G} \beta \gamma$.

Keywords: $\mathrm{Na}^{+}$, GIRK channel, $\mathrm{G}$ protein.

Voltage Dependent Ionic Currents in the Octopus Arm Muscle Fibers.

Rokni D. and Hochner B.

Dept. of Neurobiology and Interdisciplinary Center for Neural Computation, Institute of Life Sciences, Hebrew University, Jerusalem 91904

The octopus arm is a model for studying the motor control of flexible arms. In a previous study we characterized the synaptic inputs and membrane properties of the octopus arm muscle cells (Matzner et al., J. Neurophysiol. 83: 1315, 2000). In order to better understand the transformation of neural signals into muscle action, we characterized the ionic currents in dissociated muscle cells, which were then incorporated into a $\mathrm{HH}$ type model. By applying a discontinued whole cell voltage clamp technique to these isopotential cells, we found three types of ionic currents: a $\mathrm{Ca}^{++}$current, a transient $\mathrm{K}^{+}$current, and a sustained $\mathrm{K}^{+}$current. The $\mathrm{Ca}^{++}$current began activating between -40 and $-30 \mathrm{mV}$ (resting potential $\sim-70 \mathrm{mV}$ ), was sensitive to nifedipine and inactivated in a $\mathrm{Ca}^{++}$dependent manner. These properties signify this current as an L-type $\mathrm{Ca}^{++}$current. The transient $\mathrm{K}^{+}$current was quickly activated at voltages more depolarized than $-40 \mathrm{mV}$ and inactivated rapidly $(\tau \sim 10 \mathrm{~ms})$. This current was blocked by $10 \mathrm{mM} 4 \mathrm{AP}$ and was insensitive to $20 \mathrm{mM}$ TEA. Thus, this current was categorized as an A-type $\mathrm{K}^{+}$current. The sustained $\mathrm{K}^{+}$current began activating at about $-35 \mathrm{mV}$, inactivated very slowly. $(\tau \sim$ $150 \mathrm{~ms}$ ), was blocked by $10 \mathrm{mM}$ TEA and was less sensitive to 4AP. Thus, this current belongs to the delayed rectifier family. The properties of these currents were incorporated into a model cell using the Neuron modeling simulator. Current injections to the model cell elicited action potentials and oscillations comparable to those obtained in current clamp experiments, suggesting that these three ionic currents are responsible for the rich excitable behavior of the octopus arm muscle cells.

Keywords: Muscular system, $\mathrm{K}^{+}$current, $\mathrm{Ca}^{++}$current, Cephalopods

The role of ERKI/II in late phase LTP

Rosenblum K.' Voss K. ${ }^{2}$ and Bliss TVP

'Brain Research, Haifa University, Haifa 31905 , Israel. ${ }^{2}$ Division of Neurophysiology, National Institute for Medical Research, Mill Hill, London NW7 1AA,UK.

Inhibitors of extracellular regulated kinase (ERKI/II), in common with inhibitors of protein synthesis, affect long-term memory without impairing short-term memory. Previous studies have shown that ERKI/II activity modulates the initial as well as subsequent levels of LTP obtained by tetanic stimulation. To dissociate between the roles of ERKI/II in early and late plasticity we recorded the field EPSP in CA1 region of the hippocampus for at least 4 hours post-tetanus and applied the MEK inhibitor PD98059 $(38 \mu \mathrm{M})$, either $40 \mathrm{~min}$ before or immediately after the tetanus. In slices exposed to PD98059 $40 \mathrm{~min}$ before the induction of LTP there was a significant reduction in the potentiation $20 \mathrm{~min}$ post-tetanus $(32 \% \pm 8.5 \%$ versus $62 \pm 4.9 \%$, $\mathrm{p}<0.01)$. The potentiation of the drug-treated slices continued to decline over the following four hours $(26 \pm 7.1 \%$ compared to $54 \pm 4.2 \%$ in the control group after $60 \mathrm{~min}, \mathrm{p}<0.005$, and after 240 min, $18 \pm 6.3 \%$ compared to $48.3 \pm 4.7 \%$, $p<0.005$ ). In slices exposed to PD98059 immediately $(2 \mathrm{~min})$ after the tetanus there was no significant differences between control and the drug treated slices 20 or $60 \mathrm{~min}$ after induction $(62 \pm 4.9 \%$ versus $44 \pm 11 \%$ respectively at $20 \mathrm{~min}, \mathrm{p}>0.05$, and $54.7 \pm 4.2 \%$ versus $41.6 \pm 7 \%$ at $60 \mathrm{~min}, \mathrm{p}>0.05)$, but potentiation was significantly greater in control than in drug treated slices when measured 240 min post induction $(48 \pm 4.6 \%$ versus $18.5 \pm 6.3 \%, \mathrm{p}<0.005)$. Thus, ERKI/II activity is needed for the full expression of both early and late LTP.

Keywords: Long term memory, Synaptic plasticity, Gene induction 


\section{A physical liquid processor}

Rotem A. Melamed O. and Markram H.

Dept. of Neurobiology, Weizmann Institute for Science, Rehovot

A recent model shows that the dynamical state of recurrent networks embeds a continuous input across multiple time scales, solving the fundamental problem of temporal integration. In this system, readout neurons can train to retrieve information across any combination of time-scales in order to solve complex temporal processing tasks. The dynamical state of the recurrent neural network was equated with physical perturbations in a liquid. We therefore explored whether it is possible to use an actual physical liquid as a recurrent neural network and tested the capability of such a system to process sound information. Sound was translated into a mechanical vibration, which was then used to perturb the surface of water in a chamber. The perturbations on the surface of the water were captured using a video camera. The movie of perturbations was digitized and each pixel value was used to scale the current that was injected into a parallel set of readout neurons at each moment in time. The synapses onto the readout neurons were trained using a modified perceptron learning rule, to generate a target output which represented a specific computation on the sound input. We tested the system using standard voice recognition tasks, as well as complex temporal integration and memory retrieval tasks. We will present data showing how a physical liquid can act as a universal analogue processor and show how such a system can solve complex temporal processing tasks.

Keywords: Liquid computing; Physical processor; Temporal processing; Sound and voice processing

\section{$\beta$-endorphin mediates rewarding effects of cocaine: a} neurochemical, $\mathrm{fMRI}$ and behavioral study,

Roth Deri I., Aleli M., Pelled G. ${ }^{1}$, Goelman G. ${ }^{1}$ and Yadid G. Faculty of Life Sciences, Bar-Ilan University and ${ }^{1}$ Dept of Medical biophysics and Nuclear Medicine, Hadassah Hebrew University Hospital Jerusalem

The mechanism that leads to hedonism and motivation involved in drug craving is poorly understood. $\beta$-endorphin is an endogenous opioid peptide that acts as a neuromodulator and neurotransmitter in the central nervous system. The neurons in the brain that synthesize and release $\beta$-endorphin are predominantly present in the hypothalamic arcuate nucleus (Ar.N), and projects a dense innervation to the nucleus accumbens (N.Ac). Although $\beta$-endorphin is known for its analgesic effect it also has rewarding properties. Thus, leading to the hypothesis that $\beta$-endorphin may be spontaneously released in the brain not only in response to pain thereby producing reinforcement and hedonia. We used in vivo microdialysis for concomitant assessment of regional release of $\beta$-endorphin in the brain following cocaine exposure. Our results demonstrate that acute exposure or self-administration of cocaine stimulates the release of $\beta$-endorphin in the NAcc and the Ar.N. We also used functional magnetic resonance imaging (fMRI) to correlate with our neurochemical measurements using the blood oxigenation level-dependent (BOLD) contrast fMRI technique. Our results show significant focal signal increases in the. N.Ac and the Ar.N following cocaine exposure. In order to realize the crucial role of the endorphinic system in cocaine seeking behavior, we conducted a behavioral analysis using the self administration technique. Specific lesioning of the hypothalamic endorphinic neurons abolished the cocaine-seeking behavior of the rats. These results suggest that the endorphinic system of the Ar.N may be implicated in the brain circuitry mediating cocaine-induced reinforcement

Keywords: $\beta$-endorphin, Cocaine, fMR

Brain mapping in vicinity to lesions: differential probing of tissue by functional and diffusion weighted MRI Rotshtein P. ${ }^{3}$, Gigi A. ${ }^{1,3}$, Assaf Y. ${ }^{1}$, Bleich M. ${ }^{1,3}$, Pianka P. ${ }^{1,4}$, Stein A. , Palti D. , Rider-Groswasser I. ${ }^{1,3}$, Graif M. ${ }^{1,3}$, Fried I. ${ }^{2,4}$ and Hendler T

Whol Institute for Advanced Imaging', Functional neurosurgery unit, Tel Aviv Sourasky Medical center ; Dept. of Psychology.

Sackler School of Medicine, Tel Aviv University, Tel Aviv

Functional brain mapping by MRI has evolved in the last decade into a clinical tool for pre-operative evaluation of critical function in vicinity to lesions. It aimed to minimize the risk for functional deficits due to surgical intervention. However, functional MRI (fMRI) signals depends mainly on the hemodynamic response in activated gray matter and is not indicative of white-matter organization that might be crucial for safe surgical intervention. Diffusion tensor imaging (DTI) is a well-established method for in-vivo imaging of the white matter in the human brain, but its clinical application has not been fully explored. We present our recent experience in utilizing fMRI and DTI for pre-surgical brain mapping. Ten patients suffering from various brain lesions (e.g. tumor, dysplasia) underwent mapping by fMRI and DTI as part of their pre-surgical evaluation. Functional and connectivity information obtained by combined imaging approaches in MRI: Critical functional representations (e.g. language, motor) were mapped by blood oxygenation level dependent contrast, fiber tracks were mapped by DTI and both were aligned anatomically. In some cases, significant fMRI activation was observed within a lesion suggesting the presence of viable tissue. This was supported by DTI maps showing the existence of sufficiently organized white matter near the activated region. In other cases, when activation was seen in vicinity to lesion, the DTI maps assisted in avoiding injury of functionally relevant fiber tracks. The combination of methods provides validation of the functional findings on one hand, and additional connectivity information on the other hand. It is hoped that the use of these techniques will improve brain surgery outcome by decreasing behavioral side effects.

Keywords: Pre-operative mapping, Clinical fMRI, Diffusion Tensor Imaging

Ketamine induces apoptosis in the developing brain in mice Rudin M. ${ }^{1}$, Gazit V., Tashlykov V. ${ }^{2}$, Tendler Y. ${ }^{2}$ and Katz Y. ${ }^{1,2}$ ${ }^{T}$ Dept. of Anesthesiology, HaEmek Medical Center, Afula, Israel, ${ }^{2}$ Laboratory of Anesthesia, Pain and Neural Research, Bruce Rappaport Faculty of Medicine, Technion-Israel Institute of Technology, Haifa, Israel

Programmed cell death, or apoptosis, plays a very important role in nervous system development during embryogenesis and postnatal life. The interplay of different proapoptotic and antiapoptotic factors is necessary for the regulation of the final count of neurons in the developing brain. Currently it is established that one of the key roles in synaptogenesis and neuronal survival is played by the ionotropic glutamate receptors. Activation of these receptors may change global neuronal architecture and plasticity by mediation of neuronal death. The present study demonstrates that a non-competitive NMDA receptor antagonist, which is known as a dissociative anesthetic ketamine and is widely used in the practice of anesthesiology, can result in excessive apoptosis in neonatal mice even with singledose administration. Apoptotic effect was confirmed by observation of neurodegeneration (silver stain) and DNA fragmentation (TUNEL technique). The extent of apoptotic neuronal damage was clearly dose-dependent, but a remarkable increase in the rate of apoptosis was shown even after subclinical dose of ketamine $(1.25 \mathrm{mg} / \mathrm{kg})$. Use of high-dose ketamine caused widespread apoptosis, with lack of postapoptotic potentiation of formation of new synapses (demonstrated by reduced levels of synapsins I and II) that suggests long-term neuronal damage. In spite of the above morphological changes, no gross neurobehavioral effects, as determined by righting reflex, were detected. These results indicate that administration of ketamine in obstetric and pediatric practice could be a trigger of a pathological rate of apoptotic elimination of neurons that may have long-term consequences.

Keywords: Ketamine, Apoptosis, Neurodegeneration, Synapsins.

Learning-induced enhancement of synaptic potentials in pyramidal neurons: post synaptic modifications

Saar D. ${ }^{1}$, Grossman $\mathrm{Y}^{\mathrm{P}}$ and Barkai E

Dept.s of Physiology and Morphology ${ }^{2}$, Faculty of Health

Sciences and Zlotowski Center for Neuroscience, Ben-Gurion University, Beersheva

We studied the effect of olfactory learning-induced modifications in piriform (olfactory) cortex pyramidal neurons on the propagation of PSPs. Rats were trained to distinguish between odors in pairs, in an olfactory discrimination task. Three days after training completion, PSPs were evoked in layer II pyramidal cells in piriform cortex brain slices by electrical stimulation of two pathways. Stimulation of layer Ib activated the intra-cortical fibers that terminate on the proximal region of the apical and basal dendrites. Stimulation of layer Ia activated the afferent axons that originate from the olfactory bulb and terminate on the distal apical 
dendrites. PSP rise time in neurons from trained rats was significantly shorter in both the intrinsic $(2.64+0.58 \mathrm{~ms}, \mathrm{n}=26)$ and the afferent $(3.04 \pm 0.49 \mathrm{~ms}, \mathrm{n}=17)$ pathways, compared to neurons from pseudo-trained $(2.93 \pm 0.62 \mathrm{~ms}, \mathrm{n}=53, \mathrm{p}<0.05$ and $3.66 \pm 0.98 \mathrm{~ms}, \mathrm{n}=43, \mathrm{p}<0.01$ respectively) and naive rats $(3.05 \pm$ $0.51 \mathrm{~ms}, \mathrm{n}=34, \mathrm{p}<0.005$ and $3.67+0.63 \mathrm{~ms}, \mathrm{n}=22, \mathrm{p}<0.001$ respectively). The post-burst AHP, that is generated by potassium channels probably located at the proximal region of both apical and basal dendrites, reduced the amplitude of the proximal intrinsic pathway PSPs more effectively than PSPs that were generated distally by the afferent fibers. Although AHP has smaller amplitude in neurons from trained rats, the AHP conductance shunted the PSPs more efficiently in these neurons by an average of $30 \%$ in the intrinsic pathway and $53 \%$ in the afferent pathway.

We suggest that in neurons from trained rats PSPs are generated electrotonicly closer to the soma.

Keywords: Olfactory-learning, Pyramidal neurons, PSPs, Electrotonic distance

\section{Minimal requirements for $\mathbf{G} \beta \gamma$-mediated activation of $\mathbf{G}$} protein-coupled potassium channels using a tandem tetrameric approach

Sadja R.D. and Reuveny E.

Dept. of Biological Chemistry, Weizmann Institute of Science, Rehovot 76100

$\mathrm{G}$ protein-coupled inwardly rectifying potassium channels, GIRK/Kir3.x, are important elements in maintaining the resting potential of excitable cells. The gating of these channels mainly involves the association of the $G \beta \gamma$ subunits of the $G$ protein, which are released upon stimulation of neurotransmitter receptors. In the brain, GIRK channels are responsible for slow postsynaptic inhibitory signaling and in pacemaker cells of the heart, GIRK form the muscarinic-gated atrial potassium channel ( $\mathrm{I}_{\mathrm{KACh}}$ ), responsible for slowing of the heart rate. GIRK channels are believed to function as a tetrameric complex. Each of the channel tetrameric monomers was previously found to bind the $G \beta \gamma$ subunits followed by transduction of a conformational signal via the channel transmembrane domains to open the channel. It is still not clear how many $G \beta \gamma$ subunits are required for this action or what are the minimal channel elements involved in the gating process. We employed a tandem tetrameric approach to study these questions. We constructed a series of tetramers containing at various positions channel elements from the $G$ protein independent inwardly rectifying $K$ channels and tested the ability of these tetramers to undergo gating by $G$ protein coupled receptor stimulation. Our results indicate that tetramers containing less than the full complement of $\mathrm{G} \beta \gamma$ binding domains can still undergo receptor mediated gating, suggesting that G $\beta \gamma$ gating stoichiometry is less than four.

Keywords: Ion channels, G proteins, Gating

Neuroprotective and neurorescuing properties of rasagiline and TV3326 in MPTP model of Parkinson's disease

Sagi Y ${ }^{1}$, Weinreb O. ${ }^{1}$ Weinstock M. ${ }^{2}$, and Youdim M.B.H. ${ }^{1}$

The Eve Topf and USA National Parkinson Foundation Centers of Excellence for Neurodegenerative diseases, Bruce Rappaport Family Research Institute and Dept. of Pharmacology, Faculty of Medicine, Technion, Haifa; ${ }^{2}$ Dept. of pharmacology, Hebrew University, Jerusalem

The mechanism of MPTP (1-methyl-4-phenyl-1,2,3,6tetrahydropyridine) selective dopaminergic neurotoxicity is associated with its metabolism by monoamine oxidase (MAO) $-\mathrm{B}$ to the active toxin $\mathrm{MPP}^{+}$. Selective MAO-B inhibitors, including rasagiline, protect against MPTP neurotoxicity in nonhuman primates and rodents (mice). The cholinesterase-MAO inhibitor anti-Alzheimer drug, TV3326, has been developed from the pharmacophore of the anti-Parkinson drug, rasagiline. It inhibits cholinesterase as a consequence of its carbamate moiety. This drug, on chronic treatment, is also a brain selective inhibitor of $\mathrm{MAO}-\mathrm{A}$ and $-\mathrm{B}$, while its optical isomer, TV3279, is devoid of this activity. We have examined the neuroprotective and neurorescuing properties of TV3326 and TV3279 in the mice MPTP model. Chronic i.p. administration of TV3326 (75 or 150 umole $/ \mathrm{kg})$ or TV3279 $(150 \mu \mathrm{mole} / \mathrm{kg})$ daily for 14 days prior to MPTP $(24 \mathrm{mg} / \mathrm{kg} /$ day $\times 4$ days) treatment, demonstrated total neuroprotective activity only with TV 3326 by preventing the fall in striatal tyrosine hydroxylase activity, dopamine and its metabolites DOPAC and HVA levels. This action of TV3326 can be explained by the observed inhibition of both brain MAO-A and -B ( $70 \%)$ in these animals. Similar to other nonselective MAO inhibitors, it initiates dopaminergic behavioral syndrome in response to L-dopa. Thus, besides its possible anti-Alzheimer property, TV3326 might also have anti-Parkinson activity. This is not unwarranted since both selective MAO-A (moclobemide) and MAO-B inhibitors (selegiline and rasagiline) have been shown to possess beneficial anti-Parkinson activities. Results of neurorescue by TV3326, TV3279 and rasagiline will be presented.

Keywords: MPTP, TV3326, Rasagiline, Neuroprotection

The role of GABA transporter in circadian chloride regulation in $S C N$ neurons

Sagiv N. ${ }^{1}$, Belenky $\mathrm{M}^{2}$ and Yarom $\mathrm{Y}^{1}$

Depts. of ${ }^{1}$ Neurobiology and ${ }^{2}$ Cell and Animal Biology, Institute of Life Sciences, Hebrew University, Jerusalem

The mammalian circadian clock, located in the suprachiasmatic nucleus (SCN), is composed of dansely packed GABAergic neurons. We have previously shown that GABA has a dual effect on SCN neurons, excitatory during the day, and inhibitory at night (Wagner et al. Nature 387: 598-603 [1997]). This duality has been attributed to changes in intracellular $\mathrm{Cl}$ - concentration along the circadian cycle. We have demonstrated that chloride is transpored into and out of SCN neurons by two mechanisms, the efficiency of which is objected to diurnal regulation (Wagner et al. J. Physiol (in press) [2001]). We investigated the role of the GABA transporter, GAT1, in regulating chloride concentration in SCN neurons. This transporter located in the SCN on neuronal cell bodies, axon terminal, dendrites and glia, translocates GABA along with chloride and sodium. We studied the decay time of an induced GABA current in the absence and presence of the GAT1 inhibitor SKF-89976A. The blocker caused elongation of the decay time constant, and had a larger effect on inward than on outward currents. We next investigated the influence of the blocker on the recovery of intracellular $\mathrm{Cl}$ - concentration. Loading or depletion of intracellular $\mathrm{Cl}$ - was induced by a prolonged GABA application, which generated either influx or efflux of chloride, depending on the membrane holding potential. Recovery was deduced from the change in chloride reversal potential calculated from the response to a test GABA pulse presented at $30 \mathrm{sec}$ delay after the first GABA pulse. Recovery from chloride depletion was slower in the presence of SKF-89976A. This effect was more evident during subjective night.

Keywords: GABA, SCN, Circadian, Transporter

Studying a control mechanism of the octopus arm extension using a computerized dynamic model

Sagiv R. ${ }^{1}$, Yekutieli Y. ${ }^{\prime}$, Hochner B. ${ }^{1}$ and Flash T. ${ }^{2}$ Tept. of Neurobiology, Institute of Life Science and Center for Neuronal Computation, Hebrew University of Jerusalem Jerusalem 91904, Israel; ${ }^{2}$ Dept. of Applied Mathematics, Weizmann Institute of Science, Rehovot 76100, Israel

A two dimensional dynamic model of the octopus arm was developed in an earlier study. The model includes both external forces (gravity, buoyancy and water drag force) and internal forces (muscle forces and constant volume constraint force) and it is controlled by an activation signal - a simplified neuronal command of an activation wave that travels along the arm. The model was used to study a stereotypical motion of the octopus arm - the reaching movement where a bend in the arm travels towards the tip (Sagiv. et al. Eur. J. Neurosci., Vol 12 (suppl 11) p:99 [2000]). In the current study we introduce two issues. 1. The muscle model was upgraded from a linear - spring like model to a more realistic non-linear model based on experimental data. We show that during the simulated reaching movement the fully activated muscles proximal to the bend point work mainly in an isometric manner and the muscles distal to the bend point are passive. 2. We investigate the control possibilities of the extension motion in which two parameters are used: the maximal muscle force and the time it takes the wave of activation to travel along the arm. We show that there is a relationship between these two parameters, which suggests a possible minimal force control scheme.

Keywords: Octopus, Motor-control, Dynamic-model, Muscularhydrostat. 
The transition from inter-ictal to ictal like epileptic discharges is mediated by a calcium-activated cationic current.

Schiller Y

Dept. of Neurology, Rambam Medical Center, Haifa

Epilepsy is characterized asymptomatic inter-ictal spikes and symptomatic epileptic seizures. The main difference between them lies in their duration. While inter-ictal spikes are produced by a single synchronized burst, epileptic seizures are composed of recurrent synchronized bursts lasting many seconds. The main goal of this study was to elucidate the cellular mechanisms that sustain ongoing synchronized firing during electrographic seizures. The study was performed in Bicucculine treated neocortical brain slices and all recording were performed using whole-cell patch clamp recordings from single neurons.

A prolonged depolarizing envelope followed the entire course of seizures. It had an average amplitude of $12.7 \pm 1.1 \mathrm{mV}$ an average half-width of $13.3 \pm 1.4$ seconds and reversal potential of $5.4 \pm 2.4$ $\mathrm{mV}(\mathrm{n}=24)$. It was unaffected by changing the intracellular chloride concentration or adding mGluR blockers. The seizure associated prolonged depolarization waveform (SAPDW) was dependent on the intracellular calcium concentration. The initia bursts of seizures were associated with large dendritic calcium transients (up to $70 \mu \mathrm{M}$ ). Addition of intracellular BAPTA eliminated the SAPDW. Taken together these findings indicated SAPDW was mediated by a calcium dependent cationic current Addition of extracellular Flufenamic acid (FFA, $200 \mu \mathrm{M}$ ), a pharmacological blocker of the calcium activated cationic current $\left(I_{c a n}\right)$ reversibly eliminated both SAPDW and electrographic seizures $(n=7)$. Similarly, FFA eliminated seizures in neocortical brain slices exposed to exposed to a magnesium free extracellular medium $(n=5)$. These results indicated $I_{c a n}$ was responsible for sustaining recurrent burst firing during electrographic seizures and hence mediated the transition between inter-ictal and icta epileptic discharges. Blockade of $I_{c a n}$ can serve as a novel potential target for antiepileptic treatment.

Keywords: Epilepsy, calcium, cationic current.

\section{Involvement of $\mathrm{T}$ and $\mathrm{B}$ lymphocytes in the endogenous} response evoked by glutamate toxicity

Schori H. Yoles E. and Schwartz M.

Dept. of Neurobiology, Weizmann Institute of Science, Rehovot Glutamate is a neurotransmitter in the CNS. When its concentration exceeds physiological levels it becomes cytotoxic and acts as a major mediator of toxicity in acute and chronic injuries. Our laboratory showed that the ability of CNS-injured rats or mice to cope with glutamate-induced stress is genetically controlled. After exposure to glutamate, the rate of neurona survival in C57BL/6J mice, known to be susceptible to experimental autoimmune encephalomyelitis (EAE), was significantly lower than in mice that are resistant to EAE (e.g $\mathrm{Balb} / \mathrm{c})$. In the present work we examined the role of $\mathrm{B}$ and $\mathrm{T}$ cells in CNS recovery. Using Balb/c mice we showed that after intravitreal injection of glutamate or ammonium-ferrous sulfate hexahydrate (a toxic oxygen species), the loss of retinal ganglion cells in mice devoid of mature $T$ cells (nude) was significantly greater than in matched wild-type controls. In addition, using C57BL/6J mice lacking the MHC class II chaperone invarian chain (Ii-/- mice), a mutant in which the B cells do not mature we showed that recovery in the knockout mice was significantly better than in their matched wild-type controls. These findings could be partially reversed by supplying the nude mice with splenocytes and the Ii- $/$ - mice with $\mathrm{B}$ cells derived from their matched wild-type controls. The results suggest that in EAEresistant mice, potentially toxic physiological compounds, when present in excessive amounts, can recruit and activate a T-celldependent self-protective immune mechanism, and that in EAEsusceptible mice the ability to cope with glutamate toxicity is improved in the absence of B cells.

Keywords: glutamate toxicity, retinal ganglion cells, protective autoimmunity, neurodegeneration.

The effect of the novel peptide NAPVISPQ on the aggregation of the beta-amyloid peptide

Segal-Ruder Y., Ashur-Fabian O., Skutelsky E. and Gozes I

Sackler School of Medicine, Tel-Aviv University, Tel-Aviv 69978 One of the main features of the Alzheimer's disease is the formation of insoluble deposits in specific areas in the brain. The main component of these deposits is a hydrophobic 39-43 amino acid peptide termed the beta-amyloid peptide $(A \beta)$. It had been shown in many studies that $A \beta$ deposits cause neurodegeneration.
The recently discovered protein Activity-Dependent Neuroprotective Protein (ADNP) contains a sequence of an 8 amino acids NAPVSIPQ (NAP) that has shown neuroprotective features (Ann N Y Acad. Sci. 897: 125-135 [1999]) and the A $\beta$ neurotoxin (J. Neurochem. 72: 1283-1293 [1999]). The current study was designed to find out whether NAP can block the aggregation of $A \beta$.

Methods used to evaluate aggregation: 1) Fluorimetry based on association with thioflavine T. 2) Electron microscopy. 3) A high-throughput screening method using synthetic amyloid 96well plate (Nature Biothec. 15: 258-263 [19971). This assay is based on aggregation of radio-labeled $A \beta(1-40)$ onto $A \beta(1-40)$ coated plates (Synthaloid plates). 4) Congo red staining of rat cerebral cortical cultures. The experiments were conducted using either the $A \beta$ fragment amino acids $25-35$ or the natural $A \beta 1-40$ fragment. In all the experimental paradigms, NAP had shown an ability to block $1 \mathrm{rr}$ delay A $A \beta$ aggregation peaking at two NAP against $A \beta$ may be due in part to peptide-mediated disruption of amyloid beta sheets.

Supported: ISF, BSF, Neufeld, ISOA, Gildor Chair. The Synthaloid plates were from Biosource International, a kind gift from Dr. M. Scott, The R.W. Johnson Pharmaceutical Research Institute, Spring House, PA).

Keywords: Neuroprotection, peptide, ADNP, beta amyloid.

Learning-induced reduction in post-burst AHP is mediated by activation of PKC

Seroussi Y., Brosh I. and Barkai E.

Dept. of Morphology, Faculty of Health Sciences and Zlotowsk center for Neuroscience, Ben-Gurion University, Beer Sheva We have previously shown that pyramidal neurons in the rat piriform cortex from trained rats have reduced post- burst AHP for three days after odor-discrimination learning. In the present study we examined whether this long-lasting reduction in AHP is mediated by second messenger systems

The broad-spectrum kinase inhibitor $\mathrm{H} 7(10 \mu \mathrm{M})$ increased the AHP in neurons from trained rats (form $4.24+1.24 \mathrm{mV}, \mathrm{n}=25$ in N.S.R. to $5.89+1.00, n=11$ in $\mathrm{H} 7, \mathrm{P}<0.01$ ), but not in neurons from pseudo trained $(6.02 \pm 1.76, n=20$ in N.S.R. compared with $6.60+1.53, \mathrm{n}=11$ in H7) and naive (from $5.79+1.41, \mathrm{n}=17$ in N.S.R. compared with $6.04+2.12, \quad n=12$ in H7) rats. Consequently, the difference in AHP amplitude between neurons from trained and control animals was diminished. The specific PKC inhibitor GF-109203x $(10 \mu \mathrm{M})$ had a similar effect (averaged AHP amplitudes in GF were $7.37+2.05, n=13$ in trained, $6.31+1.10, n=12$ in naive and $5.82+1.45$ in pseudo trained). The PKC activator OAG $(10 \mu \mathrm{M})$ significantly reduced the AHP in neurons from naive $(2.92+0.77, n=8)$ and pseudo trained $(3.66 \pm 1.18, \mathrm{n}=7)$ rats, but not in neurons from trained rats $(3.30+1.25, \mathrm{n}=12)$, so that the difference between the groups was abolished. The PKA-specific inhibitor H-89 $(10 \mu \mathrm{M})$ increased the AHP in neurons from all groups to a similar extent, and the difference in AHP amplitude between neurons from trained rats and controls remained

We suggest that while the post-burst AHP in piriform cortex pyramidal neurons is modulated by both PKC and PKA, a PKCdependent process maintains the learning-related reduction of AHP in these cells.

Keywords: olfactory-learning, pyramidal neurons, post-burst AHP, PKC

Activity-dependent change in large random cortical networks Shahaf G. and Marom S.

Dept. of Physiology, Faculty of Medicine, Technion, Haifa,

31096 Israel

In a recent study we have shown that large random networks of cortical neurons developing in-vitro may be "trained" to express input-output relations using a simple closed-loop arrangement in which the network has a capacity to affect its own stimulation parameters (Shahaf and Marom, $J$ Neurosci in-press; Marom and Shahaf, $Q$ Rev Biophys in-press). During the training process, the functional connectivity, defined in terms of correlated activities with various time delays, changes in response to the stimuli. We are now characterizing the nature of the change in functional connectivity in the presence of repetitive stimuli. We show that activity-dependent change in the correlation between neural activities follows a Hebbian-like rule. This Hebbian behavior is observed in correlated activities with time delays extending up to 100 milliseconds, demonstrating that the synaptic learning rule is scaleable to polysynaptic connections.

Keywords: Activity-dependence; Cortical-networks; Hebb; 
Cellular and molecular aspects of protective autoimmunity in the CNS

Shaked I., Kipnis J., Porat Z. and Schwartz M.

Dept. of Neurobiology, The Weizmann Institute of Science

Central nervous system (CNS) trauma triggers a process of continuous spread of damage termed secondary degeneration. It was previously shown in our laboratory that autoimmune $T$ cells reactive to myelin proteins prevent the spread of damage and thus confer neuroprotection on the damaged CNS tissue (Moalem G et al, Nature Medicine 5:49-55 [1999]). This experimental phenomenon was later found to be the result of boosting the body's own physiological response to CNS trauma ( Yoles et al, J Neuroscience [2001]). Recent studies demonstrated that CNS secondary degeneration correlates with susceptibility to experimental autoimmune encephalomyelitis (Kipnis $\mathrm{J}$ et al, $\mathrm{J}$ Neuroscience 21:4564-4571[2001]). In studying the mechanism of action of protective autoimmunity at the site of injury and the way in which the endogenous autoimmune response is regulated, I found that site-specific recruitment of cellular elements of the innate immune response following optic nerve crush differs amongst susceptible and resistant rat strains in regards to EAE development. I also gained insight regarding the role of autoimmune $\mathrm{T}$ cell reactive to myelin proteins in activation of cellular elements innate immune response in the CNS. Further identification of each of the participants in the beneficial inflammation that occurs at the site of the damaged CNS and their molecular regulation will lead us to further understand the mechanism of "autoimmune" neuroprotection.

Keywords: Neuroprotection, EAE, Protective autoimmunity, Innate immunity.

\section{Preliminary evaluation of oral anticonvulsant treatment in the} quinpirole model of bipolar disorder Shaldubina $A_{+}^{1}$, Einat $\mathrm{H}^{1}{ }^{1}$, Szechtman $\mathrm{H}^{2}{ }^{2}$, Shimon $\mathrm{H}^{1}{ }^{1}$ and

'Zlotowski Center for Neuroscience, Faculty of Health Sciences, Ben Gurion University of the Negev, Beersheva, Israel, ${ }^{2}$ Dept. of Psychiatry and Behavioral Sciences, McMaster University, Hamilton, Ontario, Canada

A potential model for bipolar disorder, quinpirole-induced biphasic locomotion, was used for a preliminary evaluation of behavioral effects of oral anticonvulsant treatment. Quinpirole, a $D_{2} / D_{3}$ agonist, induces a biphasic locomotor response starting with inhibition and followed by excitation, resembling the oscillating nature of bipolar disorder. The present study developed a paradigm for oral administration of anticonvulsants that resulted in therapeutic blood levels and tested the effects of treatment on the quinpirole-induced response.

Eleven days treatment with valproate (12 g/liter water), phenytoin $(6 \mathrm{~g} / \mathrm{kg}$ food), and carbamazepine $(8 \mathrm{~g} / \mathrm{kg}$ food) resulted in therapeutic blood levels and in a borderline significant reduction in quinpirole-induced hyperactivity without effects on the hypoactive phase. Valproate effects became more significant at the height of the hyperactivity response. Eleven days treatment with topiramate $(30 \mathrm{mg} / \mathrm{kg})$ resulted in a significant attenuation of quinpirole-induced hyperactivity, qualitatively similar to the effects of the other anticonvulsants.

The results suggest that mood-stabilizing anticonvulsant drugs including topiramate may attenuate quinpirole-induced hyperactivity.

Keywords: Anticonvulsant, Bipolar, Topiramate, Quinpirole.

\section{The role of the right ventromedial prefrontal cortex in} empathy

Shamay-Tsoory S.G. ${ }^{1,2}$, Tomer R. ${ }^{1,2}$, Berger B.D. ${ }^{2}$ and AharonPeretz J.

'Rambam Medical Center, P.O.B 9602, Haifa 31096, Israel'

${ }^{2}$ Dept. of Psychology, University of Haifa 31905

Patients with brain damage may show altered emotional and social behavior, such as lack of empathy in social situations, especially when the damage is located in the prefrontal cortex (PFC). However, the neuroanatomical basis of impaired empathy has not been studied in detail. The empathic response of patients with localized lesions in the prefrontal cortex $(n=25)$ was compared to responses of patients with posterior $(n=17)$ and healthy control subjects $(n=19)$. Patients with prefrontal lesions were significantly impaired in empathy as compared to posterior patients and healthy controls. However, among patients with posterior lesions, those with damage to the right hemisphere were impaired (though not as severely as patients with prefrontal lesions), whereas those with left posterior lesions displayed empathy levels similar to healthy controls. A comparison of lesion sizes of 8 patients with the most profound empathy deficit revealed that lesions in the right ventromedial area were significantly larger than the lesions in either the left or right dorsolateral region. Superimposition of lesions of these patients indicated an involvement of right ventromedial impairment in seven of eight of these patients. These findings suggest that prefrontal structures play an important part in a network mediating the empathic response and specifically that the right ventromedial cortex has a unique role in integrating cognition and affect to produce the empathic response.

Keywords: Prefrontal cortex, Empathy, Brain injury.

\section{Nonlinear population codes in correlated neural networks} Shamir M. and Sompolinsky $\mathrm{H}$.

Racah Inst. of Physics and Center for Neural Computation,

Hebrew Univ., Jerusalem, Israel 91904

It is widely accepted that information in the cortex is coded in a distributed manner; an example of which is the broad tuning of neurons in M1 hand area, to the direction of voluntary reaching movements. Although the responses of single neurons are highly stochastic, it is often assumed that that the system can overcome this noise by averaging over large populations of neurons. However, experiments show that considerable correlations exist in trial-to-trial variability of firing rates of different cells, and recent theoretical study suggests that these noise correlations limit the information capacity of the system. Hence, simple averaging schemes such as the Linear Population Vector (LPV) are inadequate for extracting information from correlated populations. This raises serious questions regarding the utility of population codes in the brain. Here we study coding of information in the variances and cross-correlations of neuronal firing, in correlated populations. We show that the information embedded in the second order statistics of the neuronal responses is not suppressed by the presence of noise correlations. However, linear readouts are inadequate for extracting this information. A simple readout model, termed the Bilinear Population Vector (BPV), is proposed for extracting information embedded in neuronal correlations and variances. This readout is a nonlinear generalization of LPV. Assuming Gaussian statistics, we show that BPV is locally optimal for discrimination tasks. Application to directional coding yields that estimation error provided by BPV decreases inversely with the size of the system while the error of LPV saturates to a finite limit due to noise correlations. Finally we investigate plausible neuronal implementations of BPV.

Supported by the Israeli Science Foundation and the US-Israel $B S F$.

Keywords: Population coding, Fisher information, Nonlinear readout.

Effects of Postoperative Pain Management on the Immune

\section{Response to Surgery}

Shavit Y. ${ }^{2}$ Beilin B. ${ }^{2}$, Bessler $\mathrm{H}^{3}$, Trabekin $\mathrm{E}^{4}$, Maybord E. ${ }^{2}$, Zeidel A. ${ }^{2}$ and Mordashov B. ${ }^{2}$

${ }^{4}$ Dept of P sychology, Hebrew University of Jerusalem; ${ }^{2}$ Dept of Anesthesiology, and ${ }^{3}$ Research Institute, Rabin Medical Center Golda-Hasharon Campus, Petah Tiqva: and ${ }^{4}$ Dept of Anesthesiology, Schneider Children's Medical Center, Petah Tiqva.

Surgery is associated with immune suppression, which could affect infection rate, healing process, and tumor metastases disseminated during surgery. This suppression is the combined result of tissue damage, anesthesia, postoperative pain, and psychological stress. The present study compared the effects of several postoperative pain management techniques on immune alterations in the postoperative period.

Patients hospitalized for abdominal surgery were randomly assigned to one of three postoperative pain management techniques: Opiates on demand (intermittent opiate regimen, IOR), patient-controlled analgesia (PCA-IV), and patientcontrolled epidural analgesia (PCEA). Postoperative pain was assessed using the visual analogue scale. Blood samples were collected before, 24,48 and $72 \mathrm{hrs}$ following surgery. The following immunological assays were performed: Production of IL-1 $\beta$, IL-2, and IL-6; natural killer cell cytotoxicity (NKCC); and lymphocyte mitogenic responses.

Patients of the PCEA group exhibited lower pain scores during coughing in the first $24 \mathrm{hrs}$ postoperatively compared with patients of the IOR and PCA groups. Mitogenic responses were 
suppressed in all groups in the first $24 \mathrm{hrs}$; and returned to preoperative values by 72 hrs in the PCEA group, but remained suppressed in the PCA group. Postoperative levels of IL-1 $\beta$ and IL-6 increased in the IOR and PCA groups, while remained almost unchanged in the PCEA group. NKCC were suppressed postoperatively in all groups; there were no significant differences among the groups.

Patients receiving an epidural mixture of opiate and local anesthetics (PCEA group) exhibited reduced suppression of lymphocyte proliferation and attenuated proinflammatory cytokine response in the postoperative period.

Keywords: Postoperative pain, Immunity, Cytokines, Opiates, Inflammation

\section{The role of opiates in infant-mother bonding in sheep}

Shavit M. ${ }^{1}$, Nowak R. ${ }^{2}$, Keller M. ${ }^{2}$ and Weller A.

Dept. of Psychology, Bar Ilan University, Ramat-Gan, Israel; 'UMR6073 CNRSIINRA/University of Tours INRA PRC, Nouzilly, France.

In sheep, the mother-young relationship is characterised by the existence of individual recognition and the rapid development of an exclusive bond. The current research examined the role of the opioid system as a possible neurochemical mechanism in the establishment of a preference by the lamb for its mother. At birth, lambs were allocated at random to one of three experimental groups ( $n=10-11 /$ group). A single i.p. injection of the opioid receptor antagonist Naltrexone $1.5 \mathrm{mg} / \mathrm{kg}$ (Nalt ${ }_{1.5}$ ) or $3 \mathrm{mg} / \mathrm{kg}$ $\left(\mathrm{Nalt}_{3}\right)$ or a saline control, was administered within minutes following birth. Ewes and their newborn lambs were left undisturbed until $24 \mathrm{~h}$ and $48 \mathrm{~h}$ of age, when lambs were tested for their choice between their mother and an alien ewe. At 24h., lambs in the Nalt $\mathrm{N}_{1.5}$ group spent less time near the mother compared to control $(\mathrm{P}<0.01)$ and $\mathrm{Nalt}_{3}$ treated lambs $(\mathrm{P}<0.05)$. Furthermore, both control and $\mathrm{Nalt}_{3}$ groups spent significantly more time near the mother than near the alien ewe $(P<0.01$ and $P=0.05$ respectively), while no statistical difference was obtained for $\mathrm{Nalt}_{15}$. Moreover, Nalt ${ }_{1.5}$ treated lambs tended to spend more time near the alien ewe. The effect of $\mathrm{Nalt}_{1.5}$ persisted at $48 \mathrm{~h}$. No other significant difference in the behavior of the lambs was observed during both tests. These results support the hypothesis that opioid receptors are involved in the establishment of the preference for the mother and that the formation of this unique bond can be impaired by a single injection of an opioid receptor antagonist at birth, depending on the dose given.

Keywords: sheep - lamb - newborn - mother - opiates -

Naltrexone

\section{Growth morphology of two-dimensional insect neural}

networks

School of physics and astronomy and ${ }^{2}$ Dept. of Zoology, Tel-Aviv University,

We are studying the morphological development of neural networks in order to understand the rules that govern network selforganization. Two-dimensional in vitro networks generated from cultured locust ganglion cells offer an attractive model system due to the large size of the neurons, and their ability to grow in relative isolation as well as to develop elaborate networks.

We focused on two aspects of network formation. First, we characterized the morphology of the single neuron, and studied the effects of neuronal vicinity on the neuron's growth pattern. Second, we investigated the dynamics of the neuronal growth. We followed the timing and sequence of events leading from single isolated neurons to an interconnected network using time lapse techniques. We tested for possible pre-determined growth patterns, and for the effect of neuronal vicinity on the basic patterns.

Our results demonstrate the presence of some predetermined growth characteristics together with a strong effect of neuronal vicinity. In the absence of external stimuli (no close neighbor cells), growth cones split, demonstrating a preferred branching angle. This preferred angle gave rise to a much wider, environment-dependent range of angles in the presence of neighboring neurons.
During the growing process, growth cones connected to self or non-self neurites. As connections stabilized, tension was generated along the neurites. The structure of the single neurons as well as the network as a whole simplified as the network matured. Fully developed networks were characterized by the generation of large cell clusters connected by nerve-like structures.

Keywords: Self-avoidance, Self-organization, Growth orphology, neural network, Culture, Locust

\section{A genetic haplotype shared by Jewish patients suffering from vacuolationg megalencephalic leukodystrophy relates to an ancestral mutation in the MLC1 gene. \\ Shinar Y., Ben Zeev B., Englister Y. and Pras E. \\ Sheba Medical Center, Tel HaShomer}

The criteria for the diagnosis of this newly defined autosomal recessive neuro-degenerating disease are: gross motor delay and megalencephaly from infancy, slow motor deterioration and a typical pathologic image of brain white matter. Twelve Israeli patients presented with variable disease severity and with heterogeneous neurological symptoms. Following the localization of the responsible gene to a $2 \mathrm{cM}$ region on chromosome $22 \mathrm{q}_{\mathrm{tel}}$ by Topco et al. we screened the DNA of the Israeli families at this interval with 8 polymorphic markers including 5 newly developed ones. Analysis of linkage between the disease and these markers narrowed the disease locus down to the 550,000 base pairs at the $\mathrm{q}_{\text {tel }}$; A maximal LOD score of 5.95 was obtained at the STS28616 marker site $(\theta=0.0)$. All of the Libyan and Turkish Jewish carrier chromosomes shared a common haplotype composed of the three most telomeric markers, suggesting a common founder. Recently Leegwater et al. identified KIAA0027 at $22 q_{\text {tel }}$ as the disease gene (MLC1) in most affected families. We have sequenced the entire coding region of this gene and detected a $291 \mathrm{G} \rightarrow \mathrm{A}$ substitution at the extremity of exon 2 in the Jewish patients. The mutation leads to the substitute of glycine by glutamic acid (G59E) within a predicted transmembrane region of the putative protein. The phenotypic disease variability in these patients clearly indicates that other factors are involved in determining the final phenotype. In an Agrawali-Indian patient of a consanguineous family we have detected a single base pair insertion within exon 2 , leading to a frameshift in translation of 13 amino acid codons. In a patient of a mixed Ashkenazi-Libyan ancestry we found the G59E mutation on the Libyan chromosome, however, no sequence variation was yet found on the Ashkenazi chromosome. Parenntal screening is now available for the Israeli affected families.

Keywords: Leukodystrophy, Linkage analysis, MLC1 mutation, Myelin

Layer I of neocortex inhibits underlying laminae.

Shlosberg D. and Amitai Y.

Dept. of Physiology, Faculty of Health Sciences, Ben-Gurion

University, Beer Sheva

Mammalian cortical layer I is essentially white matter, consisting of axons of sub- and intra-cortical origin, as well as apical dendritic tufts of pyramidal neurons. A prominent feature of layer $I$ is an extensive plexus of GABAergic axons that can be traced down to smooth stellate cells in other laminae (Martinotti cells). We postulated that the integrated effect of layer I on the underlying cell populations is an inhibitory one. Field potentials were recorded from mouse brain slices using two extracellular electrodes placed at least $1 \mathrm{~mm}$ apart in layers II-III while electrical stimulation was delivered alternatingly to the two corresponding cortical columns. Recordings were made prior to and following layer I dissection.

In control conditions, the removal of layer I did not result in any epileptic phenomena. However, larger field potential amplitudes were observed following stimulations at control-equal intensities. When inhibition was partially blocked by low concentrations of the $\mathrm{GABA}_{\mathrm{A}}$ receptor blocker picrotoxin $(5-6 \mu \mathrm{M})$, the removal of layer I resulted in a significant rise in the propagation velocity of an epileptic field potential, and a significant reduction in the threshold stimulus intensity required to generate these events. There was no change in both parameters following layer I dissection when $\mathrm{GABA}_{\mathrm{A}}$ - mediated inhibition was completely blocked (picrotoxin, $100 \mu \mathrm{M}$ ).

Our results show that removal of layer I render the underlying cortical cell populations greater excitability, implying that layer I confers a net inhibitory influence upon them.

Keywords: Epilepsy propagation, Martinotti cells, Dendritic inhibition. 
Neuroprotective Role of 2-Arachidonoyl Glycerol, an Endogenous Cannabinoid, in Brain Injury Shohami E. ', Panikashvilli D. ', Simeonidou C. ${ }^{1}$, and Mechoulam R.

Depts. of Pharmacology ${ }^{I}$ and Dept. of Medicinal Chemistry and Natural Products ${ }^{2}$, The Hebrew Univeristy School of Pharmacy, Jerusalem

The metabolic arachidonic acid cascade is triggered in the brain after injury and some of its products contribute to brain damage. However, protective mechanisms are also set in motion, and reports suggest that 2-arachidonoylglycerol (2-AG), an endocannabinoid identified in recent years, may contribute towards neuroprotection. In the present study we investigated: 1) The temporal changes of $2-A G$ in mouse brain after closed head injury (CHI). 2) The neuroprotective effect of exogenous 2-AG, and 3) the role of CB1 receptor in this effect. Methods: 1) Mice were subjected to $\mathrm{CHI}$ using a weight-drop device. At various time intervals $(1-24 \mathrm{~h})$ after CHI their brains were removed, lipid extraction and separation were performed and $2-\mathrm{AG}$ was identified and quantified using GC-MS analysis. 2) 2-AG $(0.1-10 \mathrm{mg} / \mathrm{kg}$ ) was injected after $\mathrm{CHI}$, and its effect on edema (water content), clinical recovery, infarct volume (TTC staining) and hippocampal cell viability (H\&E) were evaluated. 3). CB1 antagonist (SR$141761 \mathrm{~A})$ was injected with $2-\mathrm{AG}$ and edema was evaluated at 24h. Results: 1) A massive increase of 2-AG levels was found peaking ( $\sim 10$-fold) at $4 \mathrm{~h}$. 2) $2-\mathrm{AG}$ significantly reduced brain edema and infarct volume, improved clinical recovery and increased neuronal survival. In the presence of 2-linoleyl glycerol and 2-palmitoyl-glycerol, 2-AG improved clinical recovery even at $1 \mathrm{mg} / \mathrm{kg}$. This is known as the "entourage" effect. 3) The protective effect of 2-AG was abolished by the CB1 antagonist dose-dependently. Conclusions: We suggest that 2-AG may represent a novel endogenous neuroprotective pathway, and serve as a basis for a therapeutic approach to the untreatable condition of brain trauma.

Keywords: Traumatic brain injury; Endocannabinoid,

Neuroprotection

Failure of fluoxetine to inhibit olanzapine - induced body weight gain and leptin stimulation in schizophrenic patients Shtaif B.1, Gil-Ad I.1, Poyurovsky M.2, Nechmad A.1, Maayan R.1 and Weizman A.1

Lab Biological Psychiatry, Felsenstein Institute Petah-Tiqva 49100 and Tel-Aviv University; 2Tirat Carmel Hospital. Haifa

Weight gain and increased adipose tissue are undesired sideeffects frequently associated with the treatment of olanzapine (Zyperaxa) and other atypical neuroleptics in schizophrenic patients. The suggested mechanism for the olanzapine-induced weight gain is its serotoninergic (5-HT) and histaminergic receptor antagonistic activity. Fluoxetine (Prozac), a selective serotonin reuptake inhibitor (SSRI), enhances central 5-HT activity and was reported to lower food intake and leptin levels. The aim of the study was to evaluate the effect of combined administration of fluoxetine and olanzapine on weight gain and on hormonal profile in schizophrenic patients. Thirty schizophrenic patients (21 men and 9 woman) during first episode of schizophrenia were studied. Patients were treated with olanzapine $(10 \mathrm{mg} /$ day $)$ with either fluoxetine $(20 \mathrm{mg} /$ day $)$ or placebo for eight weeks. Body weight was registered and blood samples were taken before initiation, and at the end of the study for the determination of prolactin, cortisol, TSH, Leptin, and serum serotonin. Our data showed that olanzapine treatment caused a significant increase in body weight in both placebo and fluoxetine co-treated patients. The increase in body weight was accompanied by a significant increase in leptin levels (from 4.3 to $10.0 \mathrm{ng} / \mathrm{ml}$ and form 5.1 to $11.2 \mathrm{ng} / \mathrm{ml}$ ) in the olanzapine+placebo and olanzapine + fluoxetine groups respectively. Serum serotnin levels slightly increased after olanzapine, and significantly decreased after the combined olanzapine+ fluoxetine treatment. No significant difference was registered in prolactin, cortisol, and TSH levels. Our data suggest that SSRI are ineffective in preventing olanzapine-induced increased body weight gain, and that this undesired side -effect is independent of olanzapine 5HT2 receptor antagonistic activity.

Keywords: Olanzapine, Fluoxetine, Weight gain, Leptin.
Functional correlation maps in neocortical microcircuits Silberberg G., Wu C.Z. and Markram H.

Dept. of Neurobiology, Weizmann Institute of Science, Rehovot

The neocortex consists of a diverse set of neurons intricately and precisely interconnected to form a recurrent microcircuit. The anatomy and physiology of the individual neurons and synaptic connections have been studied extensively, but the manner in which these neurons are active with respect to each other (their functional relationship) is however, not known. We developed an approach based on correlated sub-threshold synaptic activity to explore these functional relationships. Simultaneous multiple neuron whole-cell recordings were obtained from different neurons in rat neocortical slices and synaptic input was recorded before, during and after the network was stimulated with a solution containing high $\left[\mathrm{K}^{+}\right]$and lowered divalent ions. Cross correlations of the sub-threshold membrane potential during network excitation were used to represent functional relationship between neurons. The electrophysiological identity of the different neurons was obtained from a detailed electrophysiological investigation and the anatomical identity was obtained after loading cells with biocytin and $3 \mathrm{D}$ anatomical reconstructions. The study reveals characteristic functional correlations between neurons depending on the type of neurons involved and the underlying synaptic architecture. We found that the functional correlations can also be used to predict detailed microcircuit properties. We conclude that this approach offers new potential insight into the functional structure of the neocortical microcircuit during activation.

Keywords: Multineuron recordings; Subthreshold correlations Neocortex; Microcircuitry

\section{Acoustic and linguistic priming effects interact to create efficient language processing Sinai A. and Pratt $\mathrm{H}$.}

Evoked Potentials Laboratory, Technion-Israel Institute of Technology, Haifa

Brain potentials were recorded from native Hebrew (first language) speakers that also speak English (second language), using 21 scalp electrodes. Stimuli were word pairs from seven linguistic categories: words and pseudowords in Hebrew and English, words in English and Hebrew pronounced with the other language accent and words played backwards. Acoustic and linguistic parameters (acoustic envelope, pre-voicing, vowels, consonants) were controlled and balanced.

Peak amplitudes and latencies of potentials of the response to the second word in the pair were measured, analysis of variance was conducted on peak measures in addition to low resolution electromagnetic tomography analysis (LORETA) in the time frames around the ERP peaks. Results from both analysis methods indicate early (from around $100 \mathrm{~ms}$ after stimulus onset) differences in processing of the same words according to the preceding word's category. When preceded by words played backwards those differences were greater than all other categories and resembled the mismatch negativity (MMN) component. The diffuiences were located to tempro-parietal regions.

Conclusions: Auditory processing from its early stages affected by previous context including acoustic and semantic features of the previous stimuli.

Keywords: Priming, Second language, ERP's, LORETA

Analysis of $\mathrm{M}_{2}$ muscarinic receptor knockout mice reveals the mechanism that controls acetylcholine release

Slutsky I.', Wess J., Gomeza J.', Parnas I. ${ }^{2}$ and Parnas H. ${ }^{1}$

${ }^{T}$ The Otto Loewi Minerva Center for Cellular and Molecular Neurobiology, Dept. of Neurobiology, The Hebrew University, Jerusalem, Israel; ${ }^{2}$ Laboratory of Bioorganic Chemistry, National Institute of Diabetes and Digestive and Kidney Diseases,

Bethesda, MD 20892, USA.

Neurotransmitter release (amount and kinetics) in fast synapses is considered to be controlled by $\left[\mathrm{Ca}^{2+}\right]_{\mathrm{j}}$. However, recent studies suggest that the action potential may play a direct role in determining the kinetics of release by a mechanism which involves presynaptic autoreceptors. In this study, we used the mouse phrenic-diaphragm preparation as a model system to provide direct experimental support for this novel concept. Experimental manipulations that affected $\left[\mathrm{Ca}^{2+}\right]_{i}$ greatly changed the amount but not the kinetics of acetylcholine release in wildtype mice. In contrast, in mutant mice lacking functional 
(presynaptic) $\mathrm{M}_{2}$ receptors, treatments that affected $\left[\mathrm{Ca}^{2+}\right]_{\mathrm{i}}$ strongly affected both the amount and the kinetics of transmitter release. These results provide key experimental support for the novel concept that the primary mechanism controlling the kinetics of neurotransmitter release involves presynaptic autoreceptors. Keywords: $\mathrm{M}_{2}$ muscarinic presynaptic receptor, Time-course of ACh release, Knockout mice.

\section{Trajectory modifications through practice: the emergence of} effector and context specific novel trajectories in adults Sosnik R. ${ }^{1}$, Hauptmann B. ${ }^{2}$, Flash T. ${ }^{1}$ and Karni A. ${ }^{1}$

Teizmann Inst. of Science, Rehovot, Israel; ${ }^{2}$ Humboldt University of Berlin, Berlin, Germany

When we practice a motor task, we plan our sequences of basic movement components for effective performance. To study the modification through practice of motor representation, 16 subjects (Ss) underwent training (3-4 weeks, 80-150 sessions) on sequences of handwriting-like trajectories connecting 4 target points (4 segments) on a digitizing tablet (the instructions were to move "as fast and accurately as possible"). Here we report the results of the kinematic analysis in 9 Ss showing large practice related performance gains. Initially, the task was accomplished using straight trajectories, each with a bell shaped velocity profile. However, prolonged training resulted in trajectories that were shown to be maximally smooth for pairs of targets-connecting trajectories (co-articulation). This "global planning" mode was characterized by the emergence of a single curved path (replacing a pair of straight trajectories) and a robust decrease in the error between the predicted maximally smooth movement (according to the minimum jerk model) and the actual performance. The "global planning" mode was also characterized by increased inter-trial path variance for subsequent points along the path. In transfer experiments, the new curved trajectories were not sensitive to size scaling, start or end points or to changing movement direction, but were effector (hand) and task context specific. Our results support the notion that motor planning can be modulated to a large degree by experience and suggest that new effector and context specific "movement primitives" can evolve as a function of long-term practice on a sequence of movements in adults.

Supported by Dominic-Einhorn Fund.

Keywords: Motor control, Sequence learning, Co-articulation, Movement primitives

Chronic variable stress as a potential animal model for PTSD: modulation by early life maternal care. Spreekmeester E. ${ }^{1}$, Menard J. ${ }^{1}$, Sharma S. ${ }^{1}$, Diorio J. ${ }^{1}$, Rochford $\mathrm{J} .{ }^{1}$, Meaney MJ. ${ }^{1}$ and Grauer $\mathrm{E}^{1,2}$

'Douglas Hospital Research Center, McGill Univrsity, Montreal, Canada, and Israel Institute for Biological Research, NessZiona, Israel

Chronic variable stress exposure in rats was previously examined as a potential animal model of PTSD. Variations in early life maternal care may contribute to the development of this disorder. As adults, rat offspring of mothers with low licking and grooming (low-LG) scores were previously shown to display elevated behavioral and physiological responses to an acute stress, compared to offspring of high LG mothers (high-LG). We examined long term effects of six weeks of intermittent, variable stress exposure on behavior and on corticosterone (CORT) response in high-LG and low-LG. Non-stressed low-LG showed decreased habituation rate, compared to the high-LG. Stress further decreased the habituation rate in both high-LG and lowLG. Initial reactivity to the open field was lower in stressed lowLG compared to all other groups. Low-LG showed a higher acoustic startle response compared to high-LG. Stress reversed this effect in low-LG only. On a test of defensive burying, low-LG buried more than high LG. Following stress, burying was increased in the high-LG only. Non-stressed high-LG preferred sucrose more than low-LG. Chronic stress further decreased sucrose preference in low-LG but had no effect on high-LG. In addition, previously stressed female rats showed a decrease in their licking and grooming maternal care. Finally, CORT recovery is slower in the high-LG. Chronic stress reduces the CORT response to a subsequent acute stress in both high and low-LG. These data suggest that variations in maternal care differentially affect subsequent behavioral and endocrine responses to chronic stress in adult rats and may thus interact with the development of PTSD.

Keywords: Corticosteron, Open field, Defensive burring, Sucrose preference
Homocysteine-reducing strategies in schizophrenia

Stahl Z. Levine J. and Belmaker RH.

Ben Gurion University of the Negev, Beer Sheva

Elevated plasma homocysteine has been found to be a risk factor for Alzheimer's disease as well as in cerebral vascular disease, suggesting that some risk factors can accelerate or increase the severity of several CNS disease processes. Kruman (Kruman et al, $J$ Neurosci 20:6920-6 [2000]) reported that homocysteine elicits a DNA damage response in neurons that promotes apoptosis and hypersensitivity to excitoxicity. An oral methionine load has classically and consistently been reported to exacerbate schizophrenia and is of course converted to homocysteine.

Two strategies can be used in order to lower plasma homocysteine levels. One enhances the methylation of homocysteine by exogenous administration of folate and B-12 (cobalamin). Another uses low methionine diet, thus enhancing the formation of methionine from homocysteine, leading to reduced levels of homocysteine.

Therefore, we are screening chronic schizophrenic patients in our catchment area for plasma homocysteine levels and collecting a $10 \mathrm{cc}$ blood sample for plasma homocysteine on 150 patients. The highest $25 \%$ of plasma homocysteine will be offered a treatment trial of folic acid and B-12 plus a low methionine diet or placebo tablets plus a control diet in a blind randomized fashion. The low methionine diet is similar to a vegetarian diet. The control diet will be vegetarian with supplement disguised to preserve double blind. Diet treatment will last 3 months and 10-15 patients are expected to enter and complete each arm of the study. Psychiatric and dietetic rating scales will be administered every two weeks and plasma homocysteine measured. No changes will be made in patients' ongoing neuroleptic treatment.

This poster presents homosyteine levels and preliminary results of the first stage of the survey.

Keywords: Schizophrenia, Homocysteine, Methionine low diet.

Premenstrual Cyclic Psychosis in Female Adolescents

Stein D. 1 Blumenshon R. ${ }^{2}$, and Witztum E ${ }^{3}$

Stein D. Chaim Sheba Medical Center, Tel Hashomer, ${ }^{2}{ }^{*}$ Ness Ziona Mental Health Center, ${ }^{3}$ Ben Gurion University, Beer Sheva

*Affiliated with the Sackler Faculty of Medicine, Tel Aviv University, Tel Aviv, Israel

Premenstrual cyclic psychosis is an uncommon disorder, not included under accepted definitions of functional psychoses or the premenstrual syndrome. We present three 14-15 years female adolescents who developed an acute psychosis a few days before menstruation, which resolved completely upon bleeding or several days later, only to reappear with the following cycle. An extensive laboratory work-up did not show any significant physical disturbances. Psychotropic treatment had no effect on the course of the psychosis. Treatment with steroid hormones (progesterone) in the second half of the cycle in one patient, and with a combined progesterone/estrogen contraceptive agent in another resulted in full recovery within several cycles. The third patient showed a spontaneous remission within four cycles. Remission continued in all cases after discontinuation of hormonal treatment for a period of 1-4 years. We discuss several possible etiologies for this disorder including a cycloid non-specific affective disorder, a temporary functional hypothalamic-pituitary dysfunction, and the association of the pathophysiology of premenstrual cyclic psychosis with anovulation. We also discuss the role of psychotropic and hormonal treatment in this disorder.

Keywords: Psychosis, Menstruation, Affective disorders

Function of the HSV-1 latency associated transcripts in a transgenic mouse model

Steiner I. ${ }^{\prime}$, Mador N. ${ }^{1}$ and Panet A. ${ }^{2}$

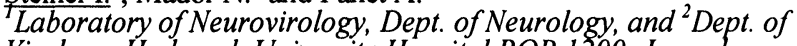
Virology, Hadassah University Hospital POB 1200, Jerusalem, 91120

Objective: To study the action mechanisms of herpes simplex virus type $1(\mathrm{HSV}-1)$ latency-associated genes during latent infection in the human nervous system. Background: During latent HSV-1 infection of human peripheral sensory ganglia limited viral transcription takes place and has been linked to the viral reactivation ability, but its action mechanisms are unknown. It was suggested that splicing of the 1.5 latency-associated transcript (LAT) is a neuronal-specific event and we have shown that the LATs repress viral replication in neurons thus facilitating establishment of HSV-1 latent infection. Methods: To study the 
action mechanisms of these genes, a transgenic mouse containing the coding sequence of the LATs was generated. Results and Conclusions: Phentotypically the transgenic mouse developed and grew well and no gross or histological abnormality was observed. Both LATs were expressed and spliced in neuronal (trigeminal ganglia, brainstem, brain) and non neuronal (liver, spleen, muscle) tissues. However, differential metabolism of these transcripts was observed in neuronal versus non-neuronal tissues, in association with the reactivation ability of the virus. At the time of abstract submission, infection experiments are on going. The findings related to the ability of the LATs to protect the viral reservoir from constant cytopathic infections and to serve as a barrier that prevents recurrent herpes infection to penetrate the same ganglion, and spread into the brain leading to herpes encephalitis will be presented.

Keywords: Herpes virus, latent infection, viral pathogenesis, nervous system

\section{Activity-dependent neuroprotective protein promotes PC12 cell survival. \\ Steingart R.A. ${ }^{1}$, Brenneman D.E. ${ }^{2}$ and Gozes I. ${ }^{1}$ \\ 'Dep. of Clinical Biochemistry, Sackler School of Medicine, Tel Aviv University, Tel Aviv 69978, Israel; ${ }^{2} S D M P, L D N$, NICHD,} NIH, Bethesda MD 20892, USA.

The human Activity-Dependent Neuroprotective protein (hADNP) is a $114 \mathrm{kD}$ novel protein which was recently cloned (J. Biol. Chem. 276:706-714 [2001]). The ADNP transcript was relatively enriched in the human cerebellum, cerebral cortex and hippocampus. In the present study, hADNP cDNA was subcloned into a vector that contains the VP22 protein, a Herpes viral protein that is able to penetrate through cell membranes. Thus, fusion VP22-proteins gain the capability of membrane penetration. The fusion protein, VP22-ADNP, was expressed in E. Coli and further purified. To address the question of cellular neuroprotection, the purified fused protein was incubated with PC12 cells and was detected within the cells after a $40 \mathrm{~min}$. incubation period. Results showed that VP22-ADNP treatment protected against $\beta$ amyloid peptide $(\mathrm{A} \beta)$ toxicity in $\mathrm{PC} 12$ cells. Furthermore, treatment with $300 \mu \mathrm{M} \mathrm{H}_{2} \mathrm{O}_{2}$ reduced $\mathrm{PC} 12$ cell survival to $47 \%$ as compared to control, while, incubation with 1 or $2 \mu \mathrm{g}$ VP22-ADNP prior to the $\mathrm{H}_{2} \mathrm{O}_{2}$ treatment significantly increased cell survival to $65 \%$ or $75 \%$, receptively. In order to examine if ADNP inhibits apoptosis caused by $\mathrm{H}_{2} \mathrm{O}_{2}$ the amount of the pro-apoptotic protein p53 was determined. $\mathrm{H}_{2} \mathrm{O}_{2}$ slightly increased p53 protein level while treatment with VP22-ADNP prior $\mathrm{H}_{2} \mathrm{O}_{2}$ significantly reduced p53 protein level. Moreover, treatment with VP22-ADNP alone reduced P53 level. These results suggest that ADNP has a role in supporting neuronal survival as well as protecting under stress conditions. Furthermore, this protection is via the inhibition of apoptosis. Support: ISF BSF Neufeld, ISOA, Gildor Chair.

Keywords: ADNP, Oxidative stress, Apoptosis

Morphological studies of synaptic plasticity and learning in rat hippocampus

Stewart M.G. ${ }^{\text {, }}$ Eyre M.D. ${ }^{1}$ and Richter-Levin G. ${ }^{2}$

Dept. of Biological Sciences, The Open University, Walton Hall, Milton Keynes. MK7 6AA UK; ${ }^{2}$ Dept. of Psychology, University of Haifa,. 31905

The effects of spatial learning on hippocampal synaptic and neuronal structure in male Sprague-Dawley rats were examined 3, 9 and $24 \mathrm{~h}$ after training in a Morris water maze. Control rats were either naïve animals or those exposed to swimming only without spatial learning. Ultrathin sections were prepared for electron microscopy and images taken from the middle molecular layer of the dentate gyrus granule cell dendritic field. Synapse density (Nv) and size of excitatory synapses were measured using unbiased counting techniques. Synapses were identified by the presence of a post-synaptic density and presynaptic vesicles, and were categorised as axo-dendritic or axo-spinous, and as perforated or non-perforated.

A significant increase occurred in non-perforated axo-spinous (NAS) synapse density in rats $9 \mathrm{~h}$ after training compared to control animals. Conversely, mean height underwent a significant but transient decrease, the reduction being greatest at $9 \mathrm{~h}$. A transient decrease also occurred in the mean apposition zone area per NAS synapse. Synapse to neuron ratios were calculated and also showed a significant increase 9 hours after training for nonperforated axo-spinous synapses, as compared to controls. These results suggest that a transient restructuring of synaptic circuits occurs as a result of training in the water maze paradigm, the data contrasting with more subtle alterations in hippocampus which we found previously 5 days after water maze training (Rusakov et al, 1997, Neuroscience 80(1),69-77), or the longer term alterations $24 \mathrm{~h}$ after LTP (Stewart et al Neuroscience 2000, 100(2), 221227).

Supported by: BBSRC grant S04435

Coordinated action of rhythmogenic networks in the neonatal rat spinal cord.

Strauss I. and Lev-Tov A

Dept. of Anatomy \& Cell Biology, The Hebrew University School of Medicine, Jerusalem,

The present work used surgical manipulations and drug application to specific regions of the spinal cord to test the longitudinal coupling between the limb and tail moving networks in isolated spinal cords of newborn rats.

Rostrocaudal coupled activation of the locomotor and tail-moving networks could be obtained by application of 5HT/NMDA with or without dopamine, or by noradrenaline (NA) with or without NMDA to a single compartment bath, or to the thoracolumbar compartment of a dual chamber bath. Caudorostral spread of rhythmic activity was observed for tens of seconds when NA or NA/NMDA were added to the sacrocaudal compartment of the split bath. Surgical manipulations of the white matter funiculi revealed that propriospinal pathways traveling in the ventral, ventrolateral and lateral funiculi contributed to the rostrocaudal coupling between the two networks.

Stimulation of sacrocaudal afferents induced a robust rhythmic activity in the sacrococcygeal cord and in the flexor-dominated segments of the lumbar cord (L1-L3). Less regular rhythm was observed under these conditions in the extensor dominated L5 segment. Pressure ejection of calcium to specific sacral segments and surgical lesions of the white matter funiculi revealed that the rhythm induced by sacrocaudal afferent stimulation in the lumbar cord depended on efficacious synaptic activation of $\mathrm{S} 3 / \mathrm{S} 4$, and on intact ventral funiculi.

In summary, coordinated limb and tail movements during various rhythmic motor behaviors are made possible by a strong coupling between the locomotor and tail-moving networks. This coupling is obtained by propriospinal pathways traveling in most of the white matter funiculi.

Keywords: Pattern generation, Spinal cord, Propriospinal pathways.

\section{Evaluation of Donepezil in the Management of Schizophrenia} with Associated Dementia

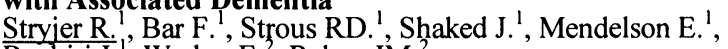
Buchiri J.', Werber E. ${ }^{2}$, Rabey JM. ${ }^{2}$

${ }^{I}$ Beer Yaakov Mental Health Center, ${ }^{2}$ Asaf Harofeh Hospital, Sackler School of Medicine, Tel Aviv University

Background: Comorbid schizophrenia and dementia is a common clinical phenomenon, however management of the coexisting illnesses remains incomplete. Donepezil, a cholinesterase inhibitor, may be beneficial in the management of symptoms of Alzheimer Disease; a disease in which cholinergic pathways in the cerebral cortex and basal forebrain are well known to be compromised. Impaired cognition in elderly schizophrenic patients has been observed to be over two-thirds, however there are no published studies reporting the use of cholinesterase inhibitors in the management of schizophrenia and associated dementia. Method: Nine patients with chronic schizophrenia (6 residual, 2 paranoid, 1 undifferentiated, mean age 67) were administered donepezil (mean dose $7.5 \mathrm{mg}$ ), mean treatment duration 20 days (for open pilot study) and 60 days (for double blind study). Patients were evaluated with MMSE, ADAS-Cog. PANSS and the CGI scales. Results: Seven patients (4 in double blind, 3 in the pilot study) improved significantly according to the CGI. The MMSE improved from a score of 17.8 to $21.8(\mathrm{p}<0.05)$, ADAS-Cog improved from 27.5 to 21.3 . PANSS improved from 109 to $92 \quad(\mathrm{p}<0.05)$. Two patients showed no change. Conclusions: Donepezil appears to be an effective treatment for the management of comorbid schizophrenia and dementia. Schizophrenia creates a vulnerable state in which even mild neuropathological degeneration is expressed in more severe cognitive impairment. Dementia associated with chronic schizophrenia may be based on a different neuropathophysiological mechanism compared to dementia not associated with schizophrenia. Cholinergic modulation may affect both positive and negative psychotic symptoms

Keywords: Donepezil, Dementia, Schizophrenia 
Decreases in synapsins in mouse brain after head injury Tashlykov V. ${ }^{1}$, Gazit V. ${ }^{1}$, Pick CG. ${ }^{2}$ and Katz Y.

${ }^{1}$ Laboratory of Anesthesia, Pain and Neural Research, Bruce Rappaport Faculty of Medicine, Technion, Haifa; ${ }^{2}$ Dept. of Anatomy and Anthropology, Sackler Faculty of Medicine, Tel Aviv University, Tel Aviv; ${ }^{3}$ Dept. of Anesthesiology, HaEmek Medical Center, Afula

Mild traumatic brain injury (MTBI) involves numerous metabolic and biochemical processes that may eventually lead to irreversible neuronal damage and death. Signaling events occurring in synaptic terminals are believed to play important roles in either promoting or preventing neuronal cell death in various physiological settings. This process correlates with mechanical and biochemical changes of synaptic vesicle membrane proteins. Using a non-invasive closed-head plummet drop model in mice, we found that low-weight groups $(5,10$ and $20 \mathrm{~g})$ had negligible microscopic changes in neurons or glial cells, but in high-weight groups $(25$ and $30 \mathrm{~g})$ there was a prominent degree of apoptosis at $72 \mathrm{~h}$ post injury in the cingulated, temporal and frontal cortex areas. To understand the signaling events occurring in synaptic terminals due to neuronal cell death, we used Western blot analysis to measure isoforms of vesicle-binding neuronal phosphoproteins synapsins I and II (Syn Ia, Ib, IIa, IIb). Brains were removed from mice after traumatic brain injury $(n=6)$ and compared with brains obtained from non-traumatized mice $(n=6)$. We did not found any change in levels of Syn Ib and IIb. In general, Syn Ib appeared to have the most widespread distribution, which is identical with other synapse-specific proteins, meaning that there is no change in synaptic contact levels after MTBI. A decrease in Syn Ia and IIa levels in the high-weight groups $(>20 \mathrm{~g})$ may demonstrate a dysfunction of inhibitory synapses. We conclude that neurodegeneration due to MTBI is expressed by dysfunction of inhibitory synapses, without a change in synaptic contact levels.

Keywords: Neurodegeneration, Minor traumatic brain injury, Synapsins.

\section{Apoptosis in traumatic brain injury in mice}

Tashlykov V. ${ }^{1}$, Gazit V.', Pick C.G. ${ }^{2}$ and Katz Y., ${ }^{1,3}$

Laboratory of Anesthesia, Pain and Neural Research, Bruce Rappaport Faculty of Medicine, Technion, Haifa; ${ }^{2}$ Dept. of Anatomy and Anthropology, Sackler Faculty of Medicine, Tel Aviv University, Tel Aviv; ${ }^{3}$ Dept. of Anesthesiology, HaEmek Medical Center, Afula

Mild traumatic brain injury (MTBI) produces prolonged and lasting cognitive and emotional derangements, manifested as amnesia, concentration disability, depression, apathy and anxiety. We simulated MTBI state by applying a non-invasive closed-head plummet drop model in mice. Mice weighing $5,10,15,20,25$ or $30 \mathrm{~g}$ were dropped from an $80-\mathrm{cm}$ height. Mice (6 per group) were decapitated at $1,6,24,48$ and $72 \mathrm{~h}$ post injury. Brains were stained with hematoxylin-eosin (H\&E) to detect gross morphological damage and thereafter with silver staining and TUNEL to qualitatively determine degree and location of apoptosis. We also measured level of the p53 tumor suppressor that is involved in the apoptotic pathway of cell death, using Western blot analysis. The low-weight group $(5 \mathrm{~g})$ demonstrated no significant levels of damaged cells in H\&E and silver impregnation. There was a weight-dependent degree of neurodegeneration in the cingulate cortex of mice exposed to high-weight plummets $(>10 \mathrm{~g})$. Other brain areas exhibited a plateau pattern of neurodegeneration in all groups treated with weights above $15 \mathrm{~g}$. TUNEL assay correlated with our findings of silver-impregnated degenerative neurons in two high-weight groups (25 and $30 \mathrm{~g}$ ). The most prominent degree of apoptosis was shown at $72 \mathrm{~h}$ post injury in the cingulate and temporal cortex areas. p53 levels were increased in the brain of mice treated with 10,15 and $20 \mathrm{~g}$ plummets. This suggests that some portion of posttraumatic neurodegeneration is p53-dependent apoptosis, which may explain the behavioral alterations seen in posttraumatic syndrome.

Keywords: Apoptosis, Mild traumatic brain injury, Neurodegeneration.
Molecular basis of electrophysiological diversity of neocortical

interneurons
Toledo-Rodriguez M. ${ }^{1}$, Wu C.Z. ${ }^{1}$, Luo J.Y. ${ }^{1}$, Mae S.L. ${ }^{1}$, Attali B. ${ }^{2}$ and Markram $\mathrm{H}$.

${ }^{I}$ Dept. of Neurobiology, Weizmann Institute of Science, Rehovot 76100; ' Dept. of Physiology, Sackler School of Medicine, Tel Aviv University, Tel Aviv

Neocortical GABAergic neurons exhibit a daunting heterogeneity in electrophysiological properties. At least 15 major electrophysiological subclasses have been identified. This electrophysiological diversity is due to different constellations $\mathrm{K}^{+}$, $\mathrm{Ca}^{2+}$, and non-specific ion channels. Studies investigating the ion channel basis of the electrophysiological behavior have been limited to a few channels. Recently we have developed a series of single cell multiplex RT-PCR protocols that allow the simultaneous investigation for the expression of over 30 voltage activated ion channel alpha and beta subunits at the single cell level. We have included virtually every channel subunit that may play a role in shaping the neurons electrophysiological behavior. Whole-cell patch clamp recordings were obtained from interneurons in neocortical slices, a detailed electrophysiological analysis carried out in which over 50 parameters of the passive and active properties of the neurons was obtained and cytoplasm was aspirated for subsequent single cell multiplex RT-PCR. During recording, neurons were also loaded with biocytin in order to allow subsequent 3D anatomical computer reconstructions, morphometric analysis and objective anatomical classification of interneurons. We will present the results of detailed correlations between mRNA profiles of ion channels and the different electrophysiological features of the cell as well as correlations between expression patterns and anatomically defied neurons. Keywords: Single Cell RT-PCR, Ion channels, Interneurons, Electrophysiology.

Molecular profiles of anatomically and electrophysiologically characterized neocortical interneurons Toledo-Rodriguez M., Luo J.Y., Wu C.Z., Mae S.L. and Markram $\mathrm{H}$. Dept. of Neurobiology, Weizmann Institute of Science, Rehovo 76100

The neocortex is composed of a small fraction of highly diverse interneurons. These interneurons are diverse in terms of anatomical and physiological as well as biochemical properties. The biochemical properties have been assessed in terms of protein or mRNA expression profiles. Studies relating morphology and electrophysiological with molecular profiles have mostly focused on protein or mRNA for the calcium binding proteins, calbindin, parvalbumin and claretinin as well as the neuropeptides, vasoactive intestinal peptide neuropeptide $\mathrm{Y}$, somatosatin and cholecystokin. Recently we developed a single cell multiplex RTPCR protocol that allows the detection of mRNA encoding for a larger spectrum of biochemical markers in combination with a comprehensive quantitative anatomical and electrophysiological characterization of the neuron. We derived an m-Code to characterize the anatomy of the cell that comprised of about 50 parameters of the axonal and dendritic morphology obtained by morphometric analysis of $3 \mathrm{D}$ reconstructed neurons. An e-Code, with more than 50 parameters of the passive and active properties of the behavior of the neurons was used to as a biophysical marker of the cell type. The g-Code was obtained from the mRNA expression profile which extended the ahove to include substance $\mathrm{P}$, corticotropin releasing hormone, cálititonin gene related peptide, dynorphin, proenkephalin, proopiomeianocortin, nitric oxide synthase and choline acetyltransferase. A detailed correlation between the g-, e- and $\mathrm{m}$-Codes will be presented.

Keywords: Single Cell RT-PCR, Interneuron, Anatomy, Electrophysiology, mRNA.

\section{Coordinated shuttling affects post session reward} consumption and neurotransmitter metabolism Tsoory M. ${ }^{1}$, Youdim M.B.H. ${ }^{2}$ and Schuster R.

${ }^{1}$ Dept. of P sychology, University of Haifa, ${ }^{2}$ The Eve Topf and USA National Parkinson Foundation Centers of Excellence for Neurodegenerative Disease and Dept. of Pharmacology, The Bruce Rappaport Faculty of Medicine, Technion, Hifa

To explain why and how cooperation occurs, an 'INDIVIDUAL' perspective focuses on individual behavior and outcomes, while a 'SOCIAL BEHAVIOR' perspective focuses on the influence of the presence and behaviors of participants. We study the latter using a model in which pairs of laboratory rats are rewarded with 
a saccharine solution for coordinating shuttles in a shared chamber with unrestricted social interaction. Previous research has shown that coordination and post-session consumption of the reward solution (PSRC) are both influenced by social interaction. We report here on evidence for adaptive changes in neurotransmitter systems selectively associated with social cooperation. A threestage procedure was used: 1) baseline PSRC was measured in five groups maintained on water deprivation; 2) three groups were rewarded for individual shuttling; 3 ) the same three groups were rewarded as follows: COOP-pairs were rewarded for coordinated shuttles; SOC- pairs were together but rewarded independently for individual shuttling; INDIV- continuation of Stage 2. A fourth group, IND-NC, received rewards not contingent on any behavior. The fifth group, CONT, were never rewarded and maintained in home-cage conditions. PSRC was measured throughout all stages. The COOP group alone developed coordinated shuttling and an increase in post-session reward consumption from Stages 2 to 3 , demonstrating selective effects of coordinated shuttling and supporting the 'SOCIAL' perspective. These data also point to physiological systems underlying cooperation. Results from HPLC analysis examine whether coordinated behavior is associated with adaptive changes in dopamine, noradrenaline and serotonin function in the frontal cortex, hippocampus and striatum. Keywords: Laboratory rat, Laboratory model, Social behavior, Coordinated behaviors, Social reward.

Plasmin modulates the axon guidance properties of $F$-spondin Tzarfati-Majar V., Feinstein Y. and Klar A.

Dept. of Anatomy and Cell Biology, Hebrew University -

Hadassah Medical School, Jerusalem

The floor plate has a profound influence on the initial axonal trajectory of various embryonic neurons. The commissural neurons are directed toward the floor plate by a diffusable molecule emanating from the floor plate. Motor neurons extend axons away from the floor plate due to repellent molecules present in the ventral neural tube. F-spondin, a gene expressed in the floor plate encodes secreted guidance protein. F-spondin plays a dual rule in patterning axonal trajectory in the spinal cord. It promotes outgrowth of commissural axons and inhibits outgrowth of motor axons. The carboxyl half of F-spondin contains 6 thrombospondin type 1 repeats (TSR). The TSR domain is processed in vivo. Serine proteases activators, implicated in a variety of processes during neurogenesis, including cell migration, axon outgrowth, and synapse elimination, are also expressed in the floor plate during embryonic development. We demonstrate that plasmin cleaves F-spondin at its carboxyl terminus. By using nested deletion proteins, and mutating potential plasmin cleavage sites, we have identified two cleavage sites - the first between the $5^{\text {th }}$ and $6^{\text {th }}$ TSR repeats, and the second at the $5^{\text {th }}$ TSR repeat. The cleaved products of F-spondin have different properties and activities. The TSR 1-4 fragment, do not bind the extracellular matrix (ECM) and inhibit outgrowth of motor axons, while the $5^{\text {th }}$ and $6^{\text {th }}$ TSR bind ECM and promotes outgrowth. Thus, plasmin controls the dual activity of F-spondin by modulating F-spondin interaction with the ECM, and demarcates between the outgrowth promoting and inhibiting domains of F-spondin.

Keywords: Axon guidance, Plasmin, Extracellular matrix.

Neuronal adaptation in auditory cortex

Ulanovsky N., Ahdut L. and Nelken I.

Dept. of physiology, Hebrew University - Hadassah Medical School, and the Interdisciplinary Center for Neural Computation, Hebrew University.

Neuronal adaptation has been only very sparsely studied in the auditory system, although the lability of the responses of auditory neurons has been repeatedly noticed. To address this question, we presented 7 halothane-anesthetized cats with an oddball paradigm : blocks of tones at a standard frequency, in which tones with a deviant frequency were embedded. The probability ratios of standard/deviant $(90 / 10 \%, 70 / 30 \%$ and a control $50 / 50 \%$ case) and the frequency difference $\mathrm{df}(44 \%, 10 \%, 4 \%)$ were manipulated. The activity of single neurons from primary auditory cortex (AI) and auditory thalamus (MGB) was recorded.

We found that neurons in AI adapt differentially to the two frequencies, so that responses to the standard frequency are more depressed than responses to the deviant frequency. This effect is positively correlated with $\mathrm{df}$ magnitude and negatively correlated with deviant probability. Moreover, there is a significant effect even for $\mathrm{df}=4 \%$, which is an order of magnitude smaller than the width of typical frequency tuning curves .

In MGB we observed much fewer neurons showing differential adaptation, and the effect was much weaker than in AI, suggesting that the adaptation is primarily cortical in origin.

The observed frequency-specific adaptation is reminiscent of mismatch negativity (MMN), an important EEG component implicated in change detection in oddball paradigms. We suggest that our data are the correlate of MMN at the single neuron level. More generally, these data show a strong form of stimulus-specific adaptation in auditory cortex. In contrast with visual analogs, we show super-resolution in frequency, the first such demonstration in the auditory system.

Keywords: Adaptation, Auditory cortex, Super-resolution,

Mismatch negativity.

Seizures and alterations in peripheral-type benzodiazepine receptor protein components in the rat brain due to systemic kainic acid injections are attenuated by PK 11195.

Veenman L. ${ }^{3}$,eschiner S. ${ }^{1}$, Spanier I., Weisinger G. ${ }^{2}$

Weizman A. ', and Gavish M. ${ }^{1}$

${ }^{I}$ Rappaport Family Institute for Research in the Medical Sciences, Technion, Haifa (MG, SL, I.S, LV); ${ }^{2}$ The Endocrine Institute, Tel Aviv Sourasky Medical Center, Tel Aviv (GW); ${ }^{3}$ Geha Mental Health Center, Felsenstein Medical Research Center, Rabin Medical Center, Beilinson Campus, Petah Tiqva (AW)

Peripheral-type benzodiazepine receptors (PBR) are located in glial cells in the brain and in peripheral tissues. Mitochondria form the primary subcellular location for PBR. Functional PBR appear to require at least three components: an isoquinoline binding protein, a voltage dependent anion channel, and an adenine nucleotide carrier. In the present study, rats received intraperitoneal kainic acid injections, which are known to cause seizures, neurodegeneration, hyperactivity, gliosis, and a 5 fold increase in PBR ligand binding density in the hippocampus. In the forebrain of control rats, hippocampal voltage dependent anion channel and adenine nucleotide carrier abundance was relatively low, while isoquinoline binding protein abundance did not differ between hippocampus and the rest of the forebrain. One week after kainic acid injection, isoquinoline binding protein abundance was increased more than 20-fold in the hippocampal mitochondrial fraction. No significant changes were detected regarding hippocampal voltage dependent anion channel and adenine nucleotide carrier abundance. Pretreatment with the isoquinoline PK11195, a specific PBR ligand, attenuated the occurrence of seizures, hyperactivity, and increases in isoquinoline binding protein levels in the hippocampus, which usually follow kainic acid application. These data suggest that isoquinoline binding protein may be functionally involved in the effects of kainic acid injections.

Keywords: Epilepsy, Neurodegeneration, Peripheral-type

benzodiazepine receptors.

\section{Multi-unit activity in the dorso-medial prefrontal cortex} during the extinction of learned fear in mice

Vouimba R.M. ${ }^{1}$, Garcia R. ${ }^{2}$, Baudry M. ${ }^{3}$ and Thompson R.F.

${ }^{7}$ Dept. of Psychology, Haifa University, Haifa 31905 Israel, ${ }^{2}$ Laboratoire de Psychophysiologie Universite de Nice-Sophia Antipolis, 06108 Nice, France, ${ }^{3}$ Neuroscience Program, USC, Los Angeles, CA 90089-2520, U.S.A.

A neutral explicit cue, such as a tone or a light, can become an aversive conditioned stimulus (CS), capable of eliciting freezing behavior, following its pairing with an aversive footshock. Repeated presentation of the CS in the absence of shock causes a reduction or elimination of freezing behavior to the CS. This process, called extinction of learned fear, is thought to involve cognitive learning: the CS is no longer reinforced. Recently, we showed that neurons in the dorso-medial prefrontal cortex (dmPFC) decrease their activity as a function of the degree of predictability of the CS (Garcia et al., Nature 18: 294-296 [1999]). However, dmPFC lesions have no effect on extinction learning per se (Vouimba et al., Behav Neurosci. 114:720-724 [2000]). We therefore hypothesized that dmPFC neurons may encode cognitive (i.e., the $C S$ is no longer reinforced) but not emotional (i.e., evolution in freezing levels) aspects of extinction learning. To examine this issue, spontaneous multi-unit activity in the dmPFC was recorded during extinction of conditioned freezing responses to either auditory or visual CS. 
The results show that, while freezing behavior decreased gradually over days, multi-unit activity in the dmPFC was reduced only on the first day of extinction but returned to baseline levels on the following days. The data suggest that the neurons in the dmPFC mediate cognitive functions during the extinction of learned fear. Keywords: Fear conditioning, Extinction, Multi-unit activity, Medial prefrontal cortex.

Activity-dependent neurotrophic factor (ADNF) and heat shock protein 60

Vulih I. ${ }^{1}$, Steingart R.A. ${ }^{1}$, Brodie C. ${ }^{2}$, Birk O.S. ${ }^{3}$ Brenneman D.E. ${ }^{4}$ and Gozes I. ${ }^{1}$

${ }^{1}$ Dept. of Clinical Biochemistry, Sackler School of Medicine, Tel Aviv University, Tel Aviv 69978, Israel; ${ }^{2}$ Gonda Center, Life Science, Bar llan Univesity, Ramat Gan 52900, Israel; ${ }^{3}$ Beer Sheva University, Israel: ${ }^{4}$ Molecular and Developmental

Pharmacology, LDN, NICHD, NIH, Betheda, MD 20892, USA.

Femtomolar concentrations of $\mathrm{ADNF}$ and related peptides (ADNF14 and ADNF9) provide neuroprotection. ADNF14 and ADNF9 are structurally similar to heat shock protein 60 (hsp60) that is stimulated under stress conditions. Hsp60 antibodies produce neuronal cell death that is inhibited by ADNF (J. Clin. Invest. 97: 2299-2307 [1996]; J. Mol. Neurosci. 14: 61-64 [2000]).

A working hypothesis was put forth that vasoactive intestinal peptide (VIP) causes a rapid release of intracellular hsp60 and ADNF, thereby enhancing the protective extracellular milieu (Neuroscience Letters. 249: 1-4 [1998]). The question arises whether hsp60 is processed to yield ADNF-like molecules? Results showed that astrocytes treated with antisense oligodeoxynucleotides complementary to hsp60 mRNA exhibited a significant reduction in the amount of intracellular hsp60-like protein (measured by Western blot analysis with specific hsp60 antibodies). When the same blots were treated with $\beta$-actin antibodies, no change was observed. In contrast, when ADNF-14 antibodies were used, a significant reduction in the ADNF-14,000 Dalton immunoreactive band was observed following hsp60 antisense oligodeoxynucleotide treatment. The reduction in ADNF-like immunoreactive band paralleled the reduction in hsp60-immunoreactivity. Recent experiments, with transfected glioblastoma cells, including an hsp60 expression vector, indicated that increases in hsp60 resulted in increases in ADNFlike immunoreactivity. These studies are now extended to measurements of biological activity, using either co-cultures with the C6-hsp60 expressing cells and primary cortical neurons or with purified ADNF (14,000 Dalton)-immunoreactive protein from C6-hsp60 glioma cells. In both cases, ADNF-like neuroprotection was observed, against starvation and against electrical blockade (tetrodotoxin), respectively. Thus, a direct association between hsp60 and an ADNF-like (14,000 Dalton) protein is suggested.

Support: ISF, BSF, Neufeld, ISOA, Gildor Chair.

Keywords: ADNF, hsp60, Antisense oligodeoxynucleotide, Glia

Patterns of Eye Movements During Motion Induced Blindness Wagner M.

\section{The Charles E. Smith National Institute for Psychobiology in}

Israel

Recently, Bonneh et al reported a phenomenon of "visual disappearance" observed with normal-sighted observers, in natural conditions. When a global moving pattern is superimposed on high contrast stationary stimuli, the latter disappear and reappear alternately for durations of several seconds. They termed the phenomenon "motion induced blindness" The MIB results provided by Bonneh et al showed that it is unlikely to reflect retinal suppression or sensory masking, but rather is a result of a conflict generated between cortical representations of dissociated stimuli which shifts the system dynamics into a winner-takes-all mode. Other alternative interpretations refer to attentional mechanisms, which cannot be allocated or divided between dissociated elements at the same spatial location, and at the same time, resulting in a reduced level of sustained attention, or alternatively competing attention mechanisms assigned to objects in space.

The purpose of this study was to investigate the attentional mechanisms underlying the MIB phenomenon as reflected by patterns of eye-movement. Utilizing the "EyeLink" eye-tracking system, we have detected relations between eye-movement patterns and gaze-directions, with subjects' reports of display component "disappearance" during a MIB situation. Study results have reveal affects of time schedule / eye movement dependence on target appearance and disappearance (e.g. target reappearance preceded by a specific eye movement features) We have also reveal depth perception effects, which might be reflected by specific dynamic patterns of binocular convergence. Results support the view that attentional mechanisms underlie the MIB phenomena.

Keywords: Eye-movements, MIB, Visual search 3D.

Autonomic function of $\alpha 5$ neuronal nicotinic acetylcholine receptors subunits

Wang N. . , Orr-Urtreger A. ${ }^{2}$, Chapman J. ${ }^{1,3}$, Rabinowitz R. ${ }^{1}$,

${ }^{I}$ Dept. of Physiology and Pharmacology, Sackler Medical School, Tel Aviv University, Tel Aviv; ${ }^{2}$ Genetic Institute and Dept. of Pediatrics and ${ }^{3}$ Neurology, Tel Aviv Sourasky Medical Center and the Sieratzki Chair of Neurology, Tel Aviv university, Tel Aviv

In 11 distinct subunits of neuronal nicotinic acetylcholine receptors (nAChRs, $\alpha 2-\alpha 9$ and $\beta 2-\beta 4$ ), $\alpha 5$ subunit appears to have unique properties in their sequences and their combinations with other subunits. In the present study, a series of autonomic tests were performed in mice lacking $\alpha 5$ nAChR subunit ( $\alpha 5-/-)$ and their wild-type mice to characterize the properties of $\alpha 5$ nAChR subunits in vivo. The results showed that rectal temperature changes in an ambient temperature of $21{ }^{\circ} \mathrm{C}$, during exposure to cold stress $\left(6^{\circ} \mathrm{C}\right)$ and following $30 \mathrm{mg} / \mathrm{kg}$ morphine were similar both in $\alpha 5-1-$ and wild-type mice. All the mice showed normal pupillary size. Morphine $(30 \mathrm{mg} / \mathrm{kg})$ induced mydriatic effect was similar in the two strains of mice. Heart rate were not significantly different between the $\alpha 5-/-$ and wild-type mice at rest, stressed by cage shaking, during exposure to cold stress or anaesthetized, although interestingly in both awake and anaesthetized mice the $\alpha 5-1-$ mice had a slightly higher HR. An impairment of cardiac parasympathetic ganglionic transmission was observed during high frequency vagal stimulation. Deficiency of $\alpha 5$ subunits strikingly increased the sensitivity to a low dose of hexamethonium $\left(\mathrm{C}_{6}\right)$ leading to a nearly complete blockade of bradycardia in response to vagal stimulation as well as elimination of rebound post-vagal-stimulation tachycardia. Such a dose of $\mathrm{C}_{6}$ only slightly depressed the effects of vagal stimulation in control mice. Another strikingly difference was that deficiency of $\alpha 5$ subunits significantly increased ileal contractile responses to cytisine and epibatidine (but not to dimethylphenylpiperazinium iodide and nicotine). Since it is well known that $\alpha 5$ subunits are integral parts of the normal ganglionic nicotinic receptors, their lack is well compensated for, presumably by the formation of other combinations. The results presented here suggest inhibitory effects of $\alpha 5$ subunits on affinity and sensitivity of agonists in the native receptors and imply that $\alpha 5$ subunits modulate the interactions between $\alpha 5$ and other $\alpha$ and $\beta$ subunit $\mathrm{nAChR}$ in vivo. Keywords: Autonomic nervous system, nAChRs, $\alpha 5$ subunit

The liquid neuron: a new perspective on single neuron processing

Wasserstrom A., Melamed O. and Markram H.

Dept. of Neurobiology, Weizmann Institute of Science, Rehovot

Neurons are characterized by complex dendritic arbors that provide a surface to receive several thousand inputs. Despite several decades of intensive research. ine main computational advantages of this complex dendritic structure remain obscure. A recent theoretical framework, termed "liquid computing" may provide new insight into the computational power of dendrites. Liquid computing shows how information can be extracted in realtime from a dynamical system. While recurrent neural networks were studied as "liquid circuits", this framework could in principle, also apply to any system, which exhibits complex inherent dynamics acting over multiple time scales. The characteristic of such a system is that the response is made up of a series of perturbations rather than a series of stable states (such as in attractor neural networks) and that a readout element can be constructed to read from these perturbations. In this framework, liquids are ideal for temporal integration because the current state of perturbations represents all past inputs across a continuum of time scales. The liquid therefore provides an analogue environment for real-time readout. In single neurons, voltages caused by inputs interact to potentially generate a complex series of voltage perturbations. We therefore explored whether it is possible to view single neurons as a "liquid". We explored two questions: First, whether the voltages present at different locations 
in the dendritic tree could hold information about a spectrum of temporal features of the input at any moment in time. Second, whether the temporal structure of action potential trains (representing a collapse from a very high dimensional activity state of the neuron to a single dimension) holds sufficient information about the voltage perturbation inside the neuron to allow readout neurons to extract specific information about features of the input to the neuron. We employed differential synaptic transmission with a spectrum of synaptic dynamics in an attempt to extract information from the action potential train. Simulations in NEURON, using computer reconstructed neurons obtained in experiments, as well as simulations in which readout elements are trained to extract information from these "liquid neurons" will be presented.

Keywords: Dendritic processing; Liquid neuron; Simulation

Neuroprotective and pro apoptotic gene expression profile of dopamine, R-apomorphine and EGCG in SH-SY5Y cell culture using cDNA microarray

Weinreb O., Levites Y., Mandel S. and Youdim M.B.H.

Eve Topf and US National Parkinson's Foundation Centers of Excellence for Neurodegenerative diseases, Bruce Rappaport Family Research Institute and Dept. of Pharmacology, Faculty of Medicine, Technion, Haifa

cDNA microarrays provide new prospects to study and identify various mechanisms of drug action. The purpose of this study to establish the mechanism of neuroprotective and pro apoptotic action of dopamine (DA), R-apomorphine (R-apo) and green tea polyphenol (-)-epigallocatechine-3-gallate (EGCG), which are radical scavengers. We have shown that DA, R-apo and EGCG demonstrated a bell shaped survival curve of SH-SY5Y cells. Thus at low concentrations $(1-10 \mu \mathrm{M})$ they are neuroprotective, but at high concentrations $(50-500 \mu \mathrm{M})$ they are pro apoptotic. We have examined gene expression using cDNA microarray. Total RNA was extracted from human neuroblastoma SH-SY5Y cells exposed to low neuroprotective and high toxic concentrations for 6.5 hours, followed by synthesis of first strand cDNA and hybridization to a customized cDNA expression array membrane consisting of only 25 genes related to apoptosis, survival and cell cycle pathways and confirmed by quantitative real-time PCR. After hybridization, chemiluminescence's detection was performed by ImageMaster VDS-CL. The cells treated with high toxic concentrations of $50 \mu \mathrm{M}$ R-apo and $500 \mu \mathrm{M}$ DA, exhibited increased expression of pro apoptotic genes bax caspase- 3 and the cell-cycle inhibitor gadd45. $50 \mu \mathrm{M}$ EGCG increased the expression of pro apoptotic genes bax, caspase- 6 and the cellcycle inhibitor gene p21, whereas the anti apoptotic gene bcl-2 was decreased. On the contrary, the lower neuroprotective concentrations did not affect the expression of most of the genes analyzed. We have demonstrated that these drugs induce similar patterns of gene expression at their neuroprotective or pro apoptotic concentrations.

Keywords: R-apomorphine, Dopamine, Green Tea Polyphenol (-) -epigallocatechine - 3-gallate, cDNA microarrays.

Effect of chronic oral administration of TV3326, a monoamine-oxidase-cholinesterase inhibitor on the pressor response to oral tyramine in conscious rabbits Weinstock M. ${ }^{1}$. Gorodetsky E. ${ }^{1}$, Wang $\mathrm{R}-\mathrm{H}^{1}$ Weinreb $\mathrm{O} .^{2}$ and Youdim M.B.H.

'Dept. of Pharmacology, Hadassah Medical School, Hebrew University of Jerusalem; '2Dept. of Pharmacology, Faculty of Medicine, Bruce Rappaport Family Research Institute, Eve Topf and US National Parkinson's Foundation Centers for

Neurodegenerative Diseases, Technion, Haifa

TV3326 inhibits cholinesterase (ChE) and monoamine oxidase (MAO) A and B in the brain but not intestine after chronic oral administration. It acts like an antidepressant in the forced swim test and causes a 2 -fold increase in brain 5HT. The present study determined the effect of TV3326 on the blood pressure (BP) response to oral tyramine. Tyramine and MAO inhibitors were administered via a naso-gastric tube to conscious rabbits. BP responses to tyramine $(10-100 \mathrm{mg} / \mathrm{kg})$ were obtained in each rabbit. From these, the dose needed to increase BP by $30 \mathrm{mmHg}$ $(\mathrm{ED} 30 \mathrm{mmHg})$ was determined before and after treatment with TV3326, (26 mg/kg/day) for 2 weeks; tranylcypromine (10 $\mathrm{mg} / \mathrm{kg}$ ) once; clorgyline $(1 \mathrm{mg} / \mathrm{kg} /$ day $)$ for 1 week; moclobemide $(20 \mathrm{mg} / \mathrm{kg}) 3$ times. ED30 $\mathrm{mmHg}$ for all rabbits before treatment was $76 \pm 4 \mathrm{mg} / \mathrm{kg}$. Tranylcypromine reduced this to $3.8 \pm 0.5$ $\mathrm{mg} / \mathrm{kg}$; clorgyline, to $12.8 \pm 1.6 \mathrm{mg} / \mathrm{kg}$ and TV3326 to $35 \pm 5$ $\mathrm{mg} / \mathrm{kg}(\mathrm{P}<0.01$ compared to other drugs). All 3 drugs inhibited $\mathrm{MAO}-\mathrm{A}$ in the brain by more than $90 \%$. Tranylcypromine and clorgyline also inhibited intestinal MAO by $90-95 \%$, but TV 3326 , by less than $10 \%$. The decrease in ED30mmHg by TV3326 was similar to that obtained with moclobemide, a reversible MAO-A inhibitor in clinical use, $(28 \pm 6)$. In conclusion, we suggest that like moclobemide, it may be possible to give TV 3326 to patients without diet restriction. Together with its ChE-inhibitory and neuroprotective activity, this asset makes TV3326 a potentially useful drug for the treatment of Alzheimer's disease with depression.

Keywords: MAO-A inhibitors, Antidepressants; Tyramine; Blood pressure.

A corn-oil preload does not reduce intake in preweanling Otsuka Long Evans Tokushima Fatty (OLETF) rats, a strain lacking $\mathrm{CCK}_{\mathrm{A}}$ receptors.

Weller A. ${ }^{1,2}$, Hurwitz I. ${ }^{2}$ and Tsitolovskya L. ${ }^{1}$

${ }^{7}$ Dept. of Psychology and ${ }^{2}$ Interdisciplinary Program in the Brain Sciences, Bar Ilan University, Ramat-Gan, Israel.

We have reported in 15-18-day old Sprague-Dawley rats, that preloads of corn oil decreased intake significantly compared to preloads of mineral oil and that pretreatment with the selective $\mathrm{CCK}_{\mathrm{A}}$ receptor antagonist devazepide significantly attenuates this effect (Weller et al., Physiol Behav, 62:871-874 [1997]). The current study attempted a conceptual replication by comparing intake of OLETF rats, lacking functional CCK $_{\mathrm{A}}$ receptors because of a genetic abnormality, with intake of control (Long Evans Tokushima [LETO]) rats. Gastric preloads (5\% BW) of $25 \%$ mineral oil or $25 \%$ corn oil were administered to 24 -hr deprived 19-20-day-old rats, 5 minutes before a 30 -minute intake test in which pups licked sweet, high-fat milk from the floor of a test chamber. Sham control rats did not receive a preload. Intake was assessed by percent body-weight gained. The pattern of previous results was replicated: LETO rats ingested significantly less after corn oil compared to after mineral oil preloads; This effect of corn oil was not evident in OLETF rats. In addition, after the mineral oil preload OLETF rats ingested significantly less milk than LETO controls. The results provide convergent support for the role of $\mathrm{CCK}_{\mathrm{A}}$ receptors in mediating fat-induced intake-reduction early in ontogeny. The increased responsiveness of OLETF rats to the non-nutritive yet volumetric and tactile stimulus of mineral oil requires further examination. (Supported by the US-Israel Binational Science Foundation).

Keywords: Independent ingestion, Fats, Satiety, CCK.

The role of interleukin-1 (IL-1) in tonic modulation of pain Wolf G. Y Yirmiya R. ', Iverfeldt $\mathrm{K}$. ${ }^{2}$ and Shavit $\mathrm{Y}$.

${ }^{1}$ Dept. of Psychology, Hebrew University, Jerusalem 91905, Israel; ${ }^{2}$ Dept. of Neurochemistry and Neurotoxicology, Stockholm University, Stockholm, Sweden

In this study we assessed the hypothesis that IL-1 signaling pathways are involved in tonic modulation of pain sensitivity, using two models of impaired IL-1 signaling: Mice with targeted deletion of the IL-1 receptor type 1 (IL-1r KO) and mice with transgenic overexpression of IL-1 receptor antagonist (IL-1 ra) within the brain (IL-1ra TG). Pain sensitivity was tested using the paw-flick and hotplate paradigms. In the paw-flick method, the latency to withdrawal of the hind-paw from a laser beam source is measured. In the hotplate test, the latency to either hind-paw licking or four-paw jump from a $53^{\circ} \mathrm{C}$ hotplate is measured. Each mutant strain displayed lower pain sensitivity compared with its wild type control. Paw-flick latency was 3.91 and $3.97 \mathrm{~s}$ in IL-1r $\mathrm{KO}$ and IL-1ra TG mice, respectively, compared with 3.26 and $2.66 \mathrm{~s}$, in their respective controls $(\mathrm{p}<.01)$. Hotplate latency was 32.61 and $31.58 \mathrm{~s}$ in IL-1r KO and IL-1ra TG mice, respectively, compared with 18.85 and $22.37 \mathrm{~s}$, in their respective controls $(p<.01)$. Mice with targeted deletion of the p55 TNF receptor (TNFrKO) and mice with targeted deletion of Interleukin 18 (IL18KO) showed higher pain sensitivity compared to their respective controls. These findings may also be related to the pain modulatory effects of IL-1, because TNFrKO mice may be more sensitive to IL-1, and IL-18KO mice are characterized by high TNF- $\alpha$ levels (TNF- $\alpha$ is known to induce IL-1 mediated hyperalgesia). These findings suggest that IL-1 signaling may be involved in tonic modulation of pain sensitivity.

Keywords: Interleukin-1 (IL-1), TNF, Pain 
Morphological, physiological, molecular and synaptic properties of martinotti cells in rat somatosensory cortex Wu C.Z.! Gupta A.', Wang Y. ${ }^{2}$, Toledo-Rodriguez M. ${ }^{1}$, Luo J.Y. ${ }^{1}$ and Markram $\mathrm{H}$.

${ }^{1}$ Dept. of Neurobiology, Weizmann Institute of Science, 76100 Rehovot, Israel; ${ }^{2}$ Section of Neurobiology, Yale University School of Medicine, 333 Cedar Street, New Haven, CT 06520-8001, USA Martinotti cells (MCs) are GABAergic interneurons that are a major part of the microcircuitry of the neocortex. Many of their intrinsic (anatomical, electrophysiological, molecular), as well as circuit properties are, however, poorly defined. We therefore obtained single and multineuron whole-cell patch clamp recordings from MCs, located in layers 2-6 of rat somatosensory cortex (P13-P15), in order to examine these properties and establish how MCs are integrated into the microcircuitry of the neocortex. MCs typically form horizontally extended axonal clusters (spanning several cortical columns) with spiny boutons in layer I. Some of their axonal and dendritic features differ in a layer-specific manner. MCs are electrophysiologically diverse ( $\mathrm{n}>80$ ), belonging to $\mathrm{c}-\mathrm{AC}, \mathrm{b}-\mathrm{AC}, \mathrm{c}-\mathrm{NAC}$ or $\mathrm{b}-\mathrm{STUT}$ subclasses. Biochemical expression patterns of common interneuronal markers (PV, CB, CR and NPY, VIP, CCK, SOM) were investigated by employing single cell RT-PCR $(n>50)$. MCs were found to be heterogeneous in their molecular profiles, displaying at least five different (co-) expression patterns. However, all MCs invariably expressed SOM and were never found to express VIP, PV and CR. Furthermore, some markers seemed to be expressed in a layer-specific manner. MCs were found to form different types of GABAergic synapses onto pyramidal cells (PCs) and interneurons, according to previously described organizational principles. MCs also received different types of GABAergic synapses from other interneurons, but invariably received facilitating glutamatergic synapses from PCs, which could sometimes cause the MCs to discharge APs. MCs received the highest number of putative synapses from pyramidal neurons compared to other interneurons, suggesting that MCs are perhaps the easiest to recruit during network activation. In conclusion, our data reveals the detailed anatomical, physiological, molecular and synaptic profiles for Martinotti cells and reveals interlayer differences in several parameters.

Keywords: Martinotti cells, Neocortex, Microcircuitry

Late-phase LTP in the amygdala of the anesthetized and the freely behaving rat induced by polymodal cortical input pathway stimulation

Yaniv D. ${ }^{1}$, Vouimba R.M. ${ }^{1}$, Diamond D. ${ }^{2}$ and Richter-Levin G. ${ }^{1}$ Dept. of Psychology, University of Haifa, Haifa 31905, Israel; ${ }^{2}$ Dept. of Psychology and Neuroscience Program, University of South Florida.

Although it is well accepted that the amygdala is a brain region related to conditioned fear responses, its exact role in these processes is under debate. One theory proposes that the amygdala modulates the strength of fear learning in other brain areas. A second theory hypothesizes that fear learning occurs in the amygdala. Long-term potentiation (LTP) is a physiological procedure believed to engage cellular mechanisms similar to those that underlie natural learning. Although LTP has been demonstrated in amygdala afferents, the verification of its reported durability was limited to $2-3 \mathrm{~h}$, a phase that does not require protein/RNA synthesis. In contrast, hippocampal studies showed late-phase LTP, lasting from hours to days, which is dependent upon de novo RNA and protein synthesis.

We studied LTP and its longevity in entorhinal cortex input to the basal amygdaloid nucleus, both in anaesthetized and freely moving rats. This route is hypothesized to serve formation of associations between complex sensory representations and emotional / motivational significance. An additional recording electrode was implanted in the dentate gyrus as an internal control. The results show that, both in the dentate gyrus and the amygdala, LTP lasts for at least $10 \mathrm{hr}$ in the anesthetized rat and for at least $96 \mathrm{hr}$ in the freely behaving rat. This suggests that the amygdala may play a role in consolidation/storage processes involved in complex emotional associations.

Keywords: Amygdala, Hippocampus, LTP, Protein synthesis, Fear memory.
Endogenous cytokines binding proteins are protective after brain injury

Yatsiv I. ${ }^{, 2}$ and Shohami E. ${ }^{2}$

${ }^{1}$ Pediatric Intensive Care, and ${ }^{2}$ Dept of Pharmacology, Hebrew University Hadassah Medical Center, Jerusalem

Acute inflammatory response following traumatic brain injury is an important factor in the development of secondary damage. We have earlier shown that the proinflammatory cytokines interleukin-1 (IL1- $\beta$ ) and tumor necrosis factor (TNF $\alpha$ ) are induced early (1-4 hours) after closed head injury (CHI). These cytokines were shown to exert deleterious effects during the acute post-traumatic period. IL-18 is a pro-inflammatory cytokine structurally related to the IL-1 family, previously known as IFN- $\gamma$ inducing factor, mediating a broad array of effector functions of both the innate and acquired immune system. IL-18 is constitutively expressed in human blood monocytes, and is also expressed in the normal CNS of mice, rats, and humans. We have recently reported that it is upregulated in mice brain at $7 \mathrm{~d}$ after $\mathrm{CHI}$

The effect of traumatic content on brain response threshold. Yeshurun Y. ${ }^{1,2}$, Rotshtein P. ${ }^{1,2}$, Malach R. ${ }^{3}$, Weizman T. ${ }^{1,2}$, Kahn ${ }_{1}^{1,2}$, Ben-Bashat D. ${ }^{1,2}$, Bleich A., ${ }^{1,2}$ and Hendler T., ${ }^{I}$ Depats. of imaging and psychiatry, Tel Aviv Sourasky medical center, Tel Aviv; ${ }^{2}$ Depts. of psychology and biology Sackler School of Medicine, Tel Aviv University, Tel Aviv; ${ }^{3}$ Dept. of neurobiology Weizmann Institute, Rehovot

Revival of trauma related sensations are characteristic of Post Traumatic Stress Disorder (PTSD). Such stressful experiences have been attributed to disturbed processing at the perceptual or the cognitive level. We aimed to examine the role of perceptual threshold in processing trauma related stimuli in PTSD. We assumed that 1) Traumatic experience could modify sensitivity of brain response. 2) Such change in sensitivity is content specific 3 ) Brain response threshold will differentiate between PTSD and non-PTSD groups.

Methods: 9 non-PTSD veterans with combat experience and 9 PTSD veterans participated in an fMRI study. Stimuli consisted of backward masked pictures with or without combat content. Pictures were presented for either 20,40 , or $80 \mathrm{msec}$, immediately followed by their scrambled picture for a total duration of $500 \mathrm{~ms}$, at a rate of $2 \mathrm{~Hz}$. 17 slices were acquired on a 1.5T, GE scanner. Subjects underwent psychophysical test of recognition. Data were analyzed, separately for amygdala complex and Lateral Occipital Complex (LOC).

Results: PTSD differed from non-PTSD veterans in their LOC response only at near threshold presentations, shown as a trend for interaction between stimuli duration and group $(\mathrm{p}<0.09)$. At near threshold presentations (i.e. 40 and $20 \mathrm{msec}$ ), PTSD veterans showed a larger fMRI signal for combat content than non-PTSD veterans $(\mathrm{P}<0.06,0.02$, respectively). In contrast, the amygdala complex showed an overall increased fMRI signal in PTSD compared to non-PTSD veterans that was unrelated to duration or content $(\mathrm{p}<0.01)$

Conclusion: Sensitivity of LOC to combat content points to an experience dependant modification of perception. Such modification could differentiate between PTSD and non-PTSD veterans. Hyper-activation of the amygdala may be linked to hyperarousal phenomena in PTSD.

Keywords: Post Traumatic Stress Disorder, fMRI, Lateral Occipital Complex, Amygdala Complex

Go2 G-protein mediates rapid desensitization of the response to lysophosphatidic acid in xenopus oocytes

Yitzhaki I., Peleg S., Dascal N., Shapira H. and Oron Y. Dept. of Physiology and Pharmacology, Sackler Faculty of Medicine, Tel Aviv University, Tel Aviv 699978

Stimulation of G-protein coupled receptors in Xenopus oocytes leads to the activation of phosphoinositide-phospholipase C pathway, mobilization of calcium and gating of calcium-sensitive chloride channels. We have previously described rapid desensitization of these responses by exposure to threshold concentration of agonist 5-30 sec before test challenge with optimal agonist concentration. Lysophosphatidic acid (LPA) elicits rapidly desensitizing (by $\sim 70 \%$ ) chloride responses in oocytes via stimulation of native LPA receptor(s). Approximately $60 \%$ of the LPA response appeared to be mediated by pertussis 
toxin-sensitive G-proteins. In order to study the mechanism of rapid desensitization, we selectively depleted native G-proteins by injection of specific antisense oligonucleotides (ASONs). ASONs-caused depletion of Gao2 or Gaol proteins resulted in 20 and $30 \%$ inhibition of the LPA response, respectively. While depletion of $\mathrm{G} \alpha 02$ had no effect on rapid desensitization, depletion of Gaol resulted in a major decrease of desensitization (to $\sim 20 \%$ ). Similar results were obtained with pertussis toxin. Using the activation of expressed cardiac inward rectifier potassium channel as a reporter, we demonstrated that LPA indeed activated Goo proteins at very low LPA concentrations and within 1-2 sec of exposure to the agonist. We concluded that although Goo2 mediates only $-30 \%$ of the chloride response, it's early activation by threshold LPA concentrations is responsible for $\sim 70 \%$ of rapid desensitization.

\section{Regulation and mechanism of amyloid precursor protein} processing by rasagiline and the anti Alzheimer drug ,TV3326 and its opicical isomer,TV3279

Yogev-Falach M., Amit T., Bar-Am O., Weinstock M. and Youdim M.B.H.

Eve Topf and NPF Centers of Excellence for Neurodegenerative Diseases Research and Dept. of Pharmacology, Faculty of Medicine, Technion, Haifa, and Dept. of Pharmacology, Hebrew University, Jerusalem,

Cholinesterase (ChE)-monoamine oxidase (MAO)-inhibitor TV3326, [(N-propargyl-(3R) aminoindan-5-yl)-ethyl methyl carbamate] has been developed as an anti Alzheimer drug from the anti Parkinson drug, rasagiline. TV3326 possesses both $\mathrm{ChE}$ and MAO-A and B inhibitory activities. However, its S-isomer, TV 3279, inhibits ChE only. All three drugs have neuroprotective activities against a variety of insults in cell cultures and in vivo. The role of these drugs in the regulation of APP processing has been examined using rat $\mathrm{PC} 12$ and human SH-SY5Y neuroblastoma cells and in vivo (rats and mice). TV3326, TV3279 and rasagiline significantly stimulated the release of the non-amyloidogenic $\alpha$-secretase form of soluble APP (sAPP) from both cells lines in a dose-dependent manner (1-100 $\mu \mathrm{M})$. This was blocked by the hydroxamic acid-based metalloprotease inhibitor, Ro31-9790, suggesting that the effect was mediated via $\alpha$-secretase activity. Using several signal transduction inhibitors, we showed that protein kinase C (PKC)-, mitogen-activated protein (MAP) kinase- and tyrosine kinasedependent pathways may be involved in the effect of TV3326 and TV3279 on the enhancement of sAPP release. TV3326 and TV3279 induced the phosphorylation of p44 and p42 MAP kinase, which was abolished by the specific inhibitors of MAP kinase activation PD98059 and U0126. Since the generation of SAPP precludes the formation of amyloidogenic derivatives, the demonstration, that the novel neuroprotective drugs TV3326, TV3279 and rasagiline can stimulate the non-amyloidogenic $\alpha$ secretase pathway suggests that these drugs may influence the basic pathogenic mechanisms underlying $\mathrm{AD}$ and could be of clinical importance for the treatment of the disease.

Keywords; Amyloid precursor protein , PKC, $\alpha$-secretase,

Cholinesterase-monoamine oxidase inhibitors.

Mitochondrial permeability transition (PT) pore as the site of neuroprotective activity of the anti Parkinson drug, rasagiline and TV3326.

Youdim M.B.H. ${ }^{1}$ Weinstock M. ${ }^{2}$, Maruyama W. ${ }^{3}$ and Naoi M. ${ }^{4}$ ${ }^{1}$ Eve Topf and NPF Centers of Excellence For Neurodegenerative Disease Research, and Dept. of Pharmacology, Faculty of

Medicine, Technion, Haifa; ${ }^{2}$ dept. of Pharmacology, Hebrew University of Jerusalem; ${ }^{3}$ Institute of Applied Biochemitry, Gifu, Japan; ${ }^{4}$ National Institute of Longevity Sciences, Aichi, Japan

The role of mitochondrial permeability transition (PT) pore in apoptosis, as induced by the endogenous neurotoxin, N-methyl-( $\mathrm{R}$ )-salsolinol (NMRSal), was studied using dopaminergic neuroblastoma SH-SY5Y cells in response to the antiapoptoticneuroprotective drugs rasagiline and TV3326. Induction of apoptosis by NMRSal $(100 \mu \mathrm{M})$ resulted in membrane permeability and a fall in mitochondrial membrane potential $(\triangle \Psi \mathrm{m})$, as shown by release of Rhodamin 123 from mitochondria. This was suppressed by cyclosporin A $(1 \mu \mathrm{M})$, a regulator of PT pore complex. The PT pore is composed of several proteins including $\mathrm{Bcl}-2$, hexokinase, peripheral benzodiazepine peripheral receptor and adenine nucleotide translocater. To clarify the regulation of PT by antiapoptotic protein family, $\mathrm{Bcl}-2$ was over-expressed in SH-SY5Y cells. This procedure resulted in complete prevention of the opening of $\mathrm{PT}$ pore as induced by proapoptotic NMRSal. Using isolated mitochondrial from rat liver and brain, the opening of PT pore was examined by the measurement of reduction in Rhodamine123 fluoresence. NMRSal opened the PT pore in a dose dependent fashion $(1-500 \mu \mathrm{M})$ and which was delayed by the antiParkinson drug rasagiline (1-

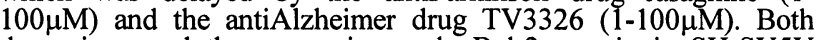
drugs increased the expression and $\mathrm{Bcl}-2$ protein in SH-SY5Y and $\mathrm{PC} 12$ cells. The results indicate that mitochondria determines the survival and death of neurons and that PT pore may be the site of neuroprotection by the drugs rasagiline and TV 3326

Keywords: Rasagline, TV3326, Bcl-2, Mitochondrial transition pore, Mitochondrial membrane potential.

\section{$\mathrm{GABA}_{\mathrm{A}}$ receptor mediates general anesthesia following} intracerebral pentobarbital microinjection

Zalkind V.I. and Devor M.

Dept. of Cell and Animal Biology, Institute of Life Sciences, Hebrew University of Jerusalem

Bilateral microinjection of barbiturates into a restricted part of the mesopontine tegmentum (the MPTA) induces atonia, bilateral loss of weight support and righting reflex, non-responsiveness to noxious pinch, EEG delta waves and apparent loss of consciousness. At low concentrations these drugs act at the barbiturate modulatory site on the $\mathrm{GABA}_{\mathrm{A}^{-}}$receptor (R). However, at the higher concentrations required to evoke anesthesia they may act non-specifically. Here we present evidence for specific action at the GABA $-\mathrm{R}$. Guide cannulae aimed at MPTA were implanted surgically in rats. After confirming that unilateral pentobarbital microinjection $(100 \mu \mathrm{g}$ in $0.5 \mu \mathrm{l})$ induced general anesthesia, muscimol $(100 \mathrm{ng}$ in $0.5 \mu \mathrm{l})$, a selective $G A B A_{A}-R$ agonist, was microinjected unilaterally through the same cannula 4-5 days later. Like pentobarbital, muscimol induced deep anesthesia, at short latency (1-3 min), including bilateral loss of righting reflex, flaccid atonia and analgesia ( 5 experiments, 4 rats). Anesthesia was not obtained following muscimol microinjection outside of MPTA (11 experiments, 8 rats). In 5 additional experiments (4 rats) bicuculline $(25 \mathrm{ng}$ in $0.5 \mu \mathrm{l})$ was microinjected unilaterally into the MPTA, followed 2-10 min later by pentobarbital at the same site. Whereas pentobarbital alone induced anesthesia, pretreatment with bicuculline prevented this. A lower concentration of bicuculline $(10 \mathrm{ng}$ in $0.5 \mu \mathrm{l})$ had a partial blocking effect. Surprisingly, microinjection of lidocaine ( $2 \%, 0.5$ $\mu l)$ did not induce anesthesia (15 unilateral trials, 11 rats), and prior microinjection of lidocaine failed to block the induction of anesthesia by pentobarbital ( 2 trial, 2 rats). The data indicate that anesthesia induced by barbiturate microinjected into MPTA is mediated by $\mathrm{GABA}_{\mathrm{A}}$ receptors.

Keywords: Anesthesia, Barbiturate, Consciousness, $\mathrm{GABA}_{\mathrm{A}}-\mathrm{R}$ Intracerebral microinjection, Muscimol

\section{NAP injections to newborns attenuate head injury-related} dysfunction in adults

Zaltzman R. ${ }^{1}$, Beni S.M. ${ }^{2}$, Giladi E. ${ }^{1}$, Pinhasov A. ${ }^{1}$, Steingart R.A. , Hill J.M. ${ }^{3}$, Brenneman D.E. ${ }^{3}$, Shohami E. ${ }^{2}$ and Gozes I. ${ }^{1}$ Clinical Biochemistry, Sackler School of Medicine, Tel Aviv University, Israel: ' Pharmacology, Hadassah Medical Center Hebrew University, Jerusalem, Israel; ${ }^{3} S D M P$,

$L D N, N I C H D, N I H$, Bethesda, MD, USA

Activity-Dependent Neuroprotective Protein (ADNP) contains a femtomolar acting neuroprotective peptide, NAP (J. Biol .Chem. 276: 708-714 [2001]; J. Pharmacol. Pharm. Exp. Therap. 293:1091-1098 [2000]; 296:57-63 [2001]; 297: 774-779 [2001]). Here, NAP was tested in a model of apolipopoprotein E-deficient mice subjected to head trauma (Neurosci. 80:1255-1262 [1997]; $J$. Neurochem.72:1283-1293 [1999]). NAP was injected (sc) for the first 3 weeks of life and at 4-months of age closed head injury was carried out. Mice injected with saline served as controls. In NAPtreated mice gliosis was less evident and neurobehavioral recovery was superior to that of controls. At 1,24 hours and one week after trauma mice were tested for performance in 10 different tasks and rated using a Neurological Severity Score (NSS), evaluating damage to motor ability, balance and alertness. Comparing NSS values revealed that NAP-pre-treated animals recovered faster and to a larger extent than saline pre-treated animals. One week after trauma, mice were subjected to a 5-day Morris water maze test evaluating learning and memory. Short-term memory processes 
were examined by measuring the time required for each mouse to find a hidden platform in the second of 2 daily trials. When observing only mice with NSS between 4 to 7 (moderate to severe injury), it was found that from the fourth testing day, the NAPtreated group showed improved performance, significantly better than their first day performance. In contrast, control group did not exhibit learning behavior in the water maze. This study indicates long-term NAP effects and future possibilities of using NAP as a prophylactic drug

Support: ISF BSF, Neufeld, ISOA, Gildor Chair.

Keywords: Trauma, Neuroprotection, Peptide, ADNP.

Rewarding and psychomotor stimulant effects of endomorphin-1 in the ventral tegmental area: dopaminedependent mechanisms?

Zangen A., Ikemoto S. and Wise R.A.

Behavioral Neuroscience, National Institute on Drug Abuse, NIH

Endomorphin-1, the presumed endogenous ligand for the $\mu$-opioid receptor, is found in neurons that project to opiate reward sites in the ventral tegmental area (VTA). Previously we demonstrated that microinjection of endomorphin-1 into the posterior VTA produces a robust locomotor stimulating effect and that rats can learn to lever-press for such microinjections. In order to learn whether these effects are dependent on the dopaminergic cells in the VTA, we induced dopaminergic lesions in the VTA of one hemisphere and compared the rewarding and locomotor stimulating effects of endomorphin-1 when injected into the lesioned versus the sham-lesioned VTA of the same rats. Microinjections of endomorphin-1 $(0.1-1.0 \mathrm{nmol})$ into the shamlesioned posterior VTA produced a robust locomotor stimulating effect, while the same doses into the lesioned VTA had no locomotor stimulating effect. On the other hand, the rats learned to lever-press for endomorphin-1 $(0.25 \mathrm{nmol} /$ infusion $)$ into both the lesioned and the sham-lesioned VTA, although the rate of endomorphin-1 self-infusions into the lesioned VTA was lower than the rate of self-infusion into the sham-lesioned VTA. These data suggest that dopaminergic cells in the posterior VTA play rolls in both the rewarding and the psychostimulant effects of endomorphin-1. While its psychostimulant effect appears to be dopamine-dependent , EM-1 appears to have a rewarding action in the VTA that is dopamine-independent.

Keywords: Endomorphine-1, Dopamine, Reward, Ventral

Tegmental Area.

The locust frontal ganglion: a central pattern generator controlling foregut motor patterns in feeding and molting

related behaviors

Zilberstein Y. and Ayali A.

Dept. of Zoology, Tel Aviv University, Tel Aviv, 69978

In the desert locust Schistocerca gregaria, ganglion (FG) plays a key role in control of foregut movements and constitutes the major source of innervation to the foregut dilator muscles. This work studies the generation and characteristics of FG motor outputs in two distinct and fundamental behaviors: feeding and molting. In an in vitro preparation, isolated from all descending and sensory inputs, the FG was spontaneously active and generated rhythmic multi-unit bursts of action potentials, which could be recorded from all efferent nerves. Intracellular recordings suggest that only a small fraction of the FG 100 neurons demonstrate rhythmic activity. Known insect neuromodulators as well as molt-related peptides were able to modulate the FG pattern in vitro. The FG motor output in vivo was relatively complex, and strongly dependent on the locust's physiological and behavioral state. Rhythmic activity of the foregut was found to depend on the amount of food present in the crop; animals with full crop demonstrated higher FG burst frequency than those with empty crop. When no feeding-related foregut pattern was observed, the FG motor output was strongly correlated with the locust's ventilation pattern. This ventilationrelated rhythm was dominant in pre-molting locusts. During the molt synchronization with the ventilation pattern can be transiently switched off revealing the endogenous (feedingrelated) FG pattern. This presumably happens during vigorous air swallowing. Our results indicate the presence of a central pattern generator network in the FG. The FG rhythmic pattern is modulated by chemical factors as well as by interactions with other pattern generating circuits.

Keywords: Frontal ganglion; CPG; Feeding; Molting; Ventilation pattern
Long term learning deficits follow minor Traumatic Brain Zohar O. ${ }^{1}$ Schreiber S. ${ }^{2}$ Getslev V. ${ }^{3}$, Schwartz JP. ${ }^{4}$ Mullins PG. and Pick CG

I Blanchette Rockefeller Neurosciences Institute, Johns Hopkins Academic and Research Building, Rockvilie, MD. ${ }^{2}$ Dept. of

Psychiatry, Tel Aviv Sourasky Medical Center and Tel-Aviv University, Sackler School of Medicine, Tel Aviv, Israel. ${ }^{3}$ Dept. of Anatomy and Anthropology, Tel-Aviv University, Sackler School of Medicine, Tel Aviv, Israel. "Neurotrophic Factors Section, National Institute of Neurological Disorders and Stroke, Bethesda, MD. ${ }^{5}$ Clinical Magnetic Resonance Research Center University of New Mexico, Albuquerque, $N$.

Victims of minor Traumatic Brain Injury (mTBI) show no clear morphological brain defects. However, those patients frequently suffer lasting cognitive and emotional difficulties including various degrees of amnesia, difficulty with concentration, depression, apathy and anxiety known as post-concussive syndrome. We adopted the non-invasive closed-head weight drop model in mice, to closely mimic real life mTBI. Following, 20, 25, or 30 gr weight drop onto the mice skull we observed no brain edema, no morphological changes to the brain as assessed by fMRI and no damage to the blood brain barrier. Non of the experimental animals showed post trauma neurological deficits. To examine the effect of mTBI on the spatial learning of these mice we used the Morris water maze 7, 30, 60 and 90 days post trauma. At each time point, the animals were tested twice a day, for 5 consecutive days. In the second trial of day five the platform was removed and the time spent in the missing platform quadrant was recorded for $45 \mathrm{sec}$. The escape latencies of all the injured mice were significantly slower than control mice $(p<0.01)$ and they spent $30 \%$ less time in the missing platform quadrant $(p<0.001)$. Moreover, the injured mice could not improve their performance beyond the second trial, regardless of the experimental parameters. This demonstrates that persistent cognitive deficits in mice, similar to the human post-concussive syndrome, can follow mTBI without any morphological damage to the brain and its surrounding tissue

Keywords: Minor Traumatic Brain Injury, Maze Learning, Mice

Immune activation during pregnancy leads to a post-pubertal emergence of an attentional deficit and a dopaminergic hyperfunction in the offspring: A novel neurodevelopmental model of schizophrenia.

Zuckerman L. ${ }^{1}$, Rehavi M. ${ }^{2}$, Nachman R. ${ }^{2}$ and Weiner I. ${ }^{1}$ Tept. of Psychology, Tel Aviv University, Tel Aviv 69978, ${ }^{2}$ Dept. of Physiology and Pharmacology, Sackler Faculty of Medicine,Tel Aviv University, Tel Aviv 69978

In recent years, prenatal exposure to viral infection has gained centrality as one of the environmental factors etiologically related to schizophrenia. It has been suggested that maternal immune response to viral infection, and in particular pro-inflammatory cytokines released by the maternal immune system, may interfere with normal fetal brain development and thus predispose to schizophrenia. To test this hypothesis in rats, we studied the effects of maternal immune activation on in vitro striatal dopamine release and on latent inhibition (LI) in juvenile and adult male and female offspring. LI refers to retarded conditioning to a stimulus as a consequence of its repeated inconsequential preexposure, and disrupted LI models an attentional deficit in schizophrenia. Poly IC which simulates an in vivo viral response by inducing the release of pro-inflammatory cytokines, was administered on gestation day 15 . When tested at prepubertal age the offspring of saline and Poly IC-treated dams exhibited normal $\mathrm{LI}$ and there were no differences in basal and $\mathrm{KCl}$-induced striatal dopamine release. In contrast, adult offspring of Poly IC dams failed to show LI, and this was reversed by antipsychotic treatment. Likewise, $\mathrm{KCl}$-induced dopamine release was increased in the adult offspring. Thus, immune activation during pregnancy in rats led to a post-pubertal emergence of phenomena reminiscent of schizophrenia in terms of a putative inducing factor, temporal course, neurotransmitter dysfunction, cognitive impairment and responsiveness to treatment, supporting the hypothesis that maternal immune response to infection may be responsible for the interaction between maternal infection during pregnancy, altered neuronal development and adult schizophrenia.

Keywords: Maternal immune activation; Latent inhibition; Invitro striatal dopamine release, Neurodevelopment 

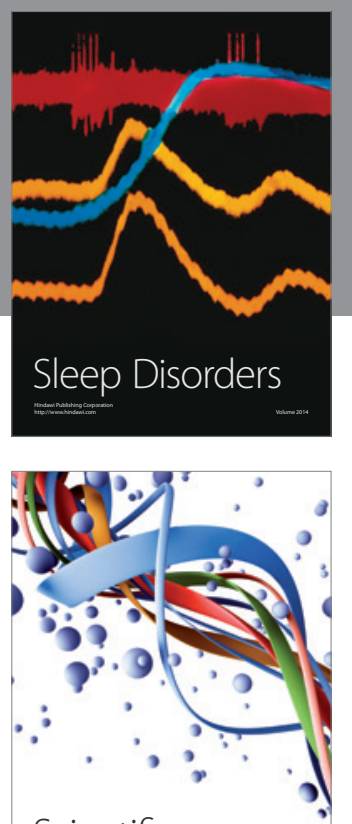

Scientifica
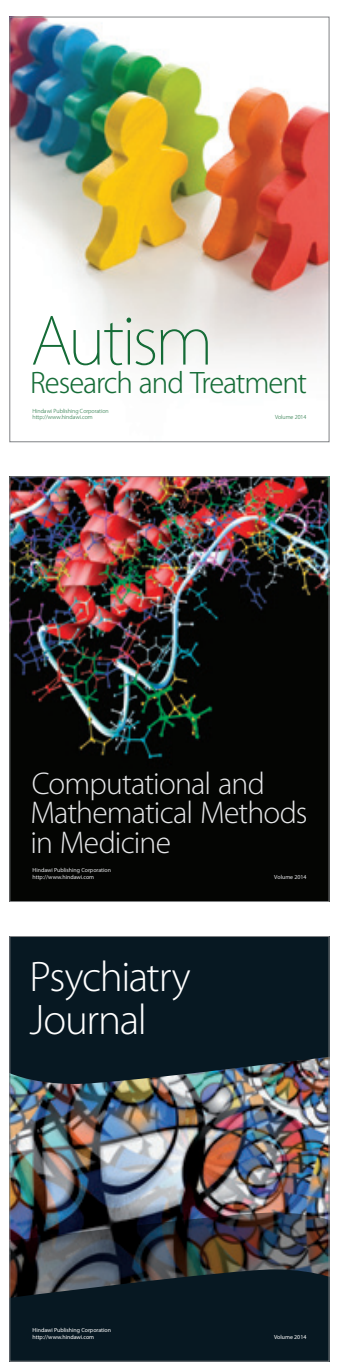
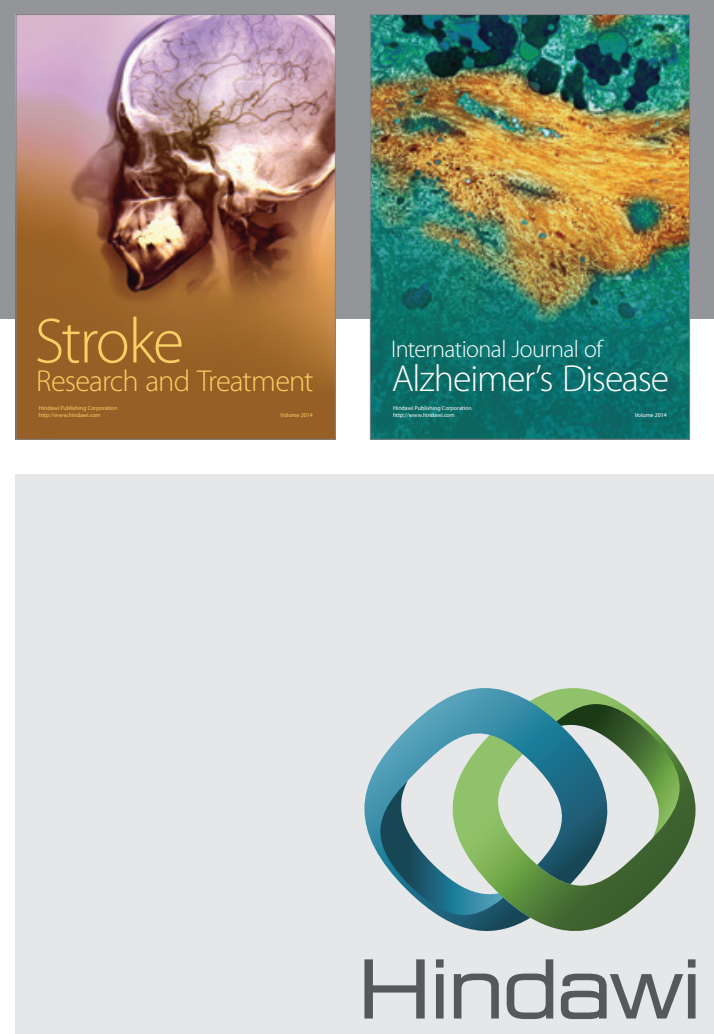

Submit your manuscripts at

http://www.hindawi.com
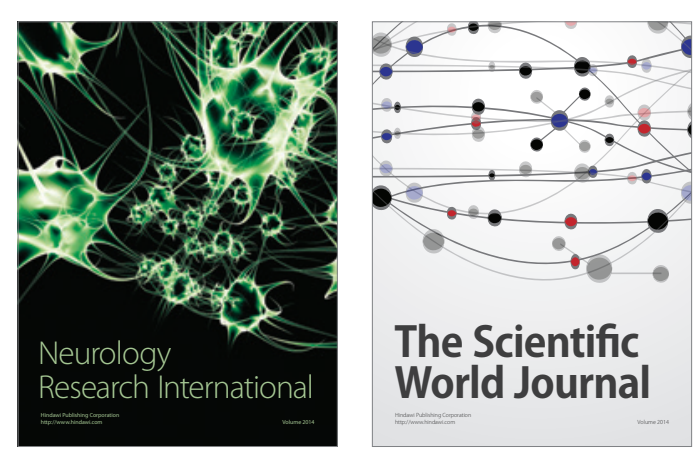

The Scientific World Journal

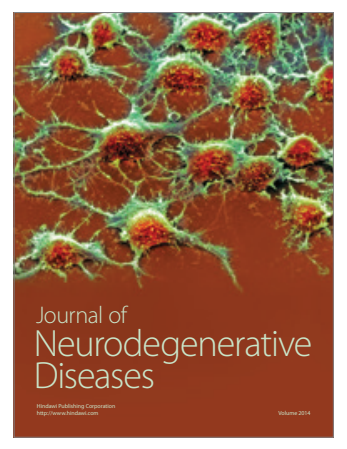

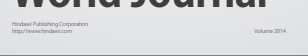

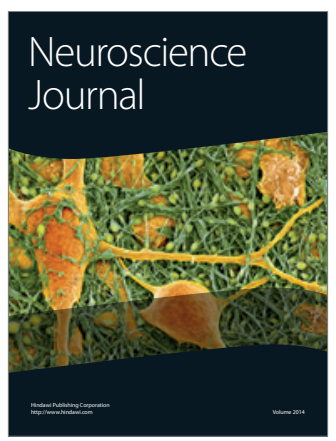

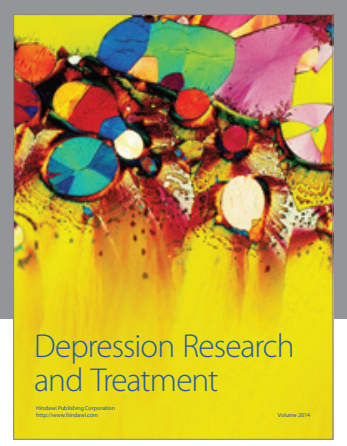
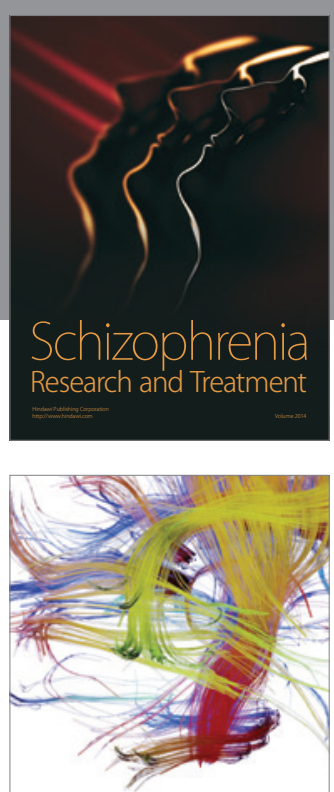

Brain Science

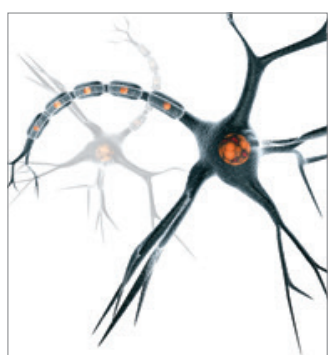

Neural Plasticity
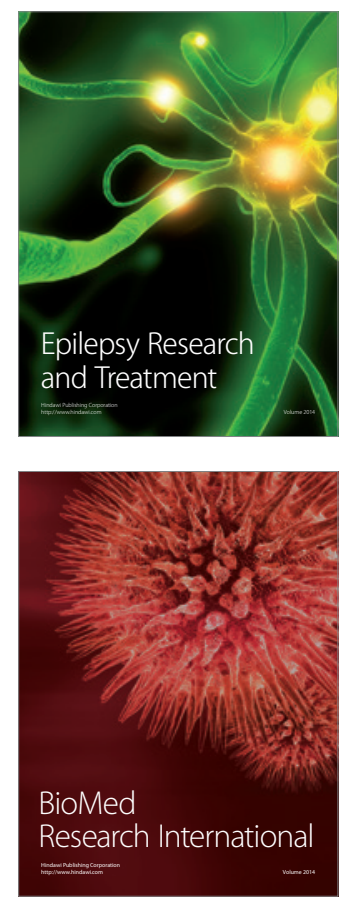

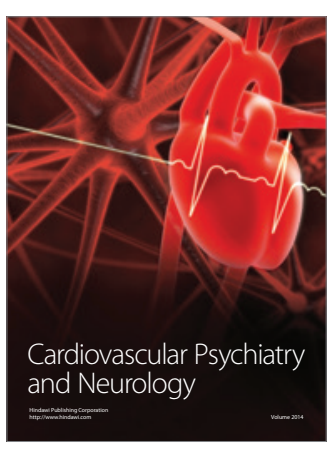

Parkinson's

Disease
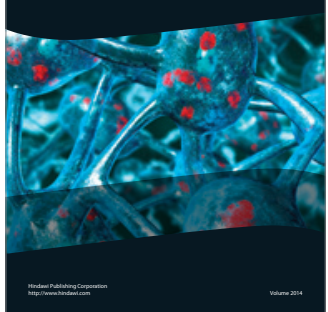